Pacific Journal of Mathematics

NONSOLVABLE FINITE GROUPS ALL OF WHOSE LOCAL

JuN Gross Torso 


\title{
NONSOLVABLE FINITE GROUPS ALL OF WHOSE LOCAL SUBGROUPS ARE SOLVABLE, IV
}

\author{
JoHN G. THOMPSON
}

\begin{abstract}
In this paper, the simple $N$-groups are classified for which $e \geqq 3$ and $2 \in \pi_{4}$. This latter condition means that a Sylow 2-subgroup contains a normal elementary abelian subgroup of order 8 and does not normalize any nonidentity odd order subgroup.
\end{abstract}

As in III, the proofs rely heavily on the fact that many subgroups of odd order are contained in just one maximal subgroup. The numbering of the sections is a continuation of III. The bibliographical references are to be found at the end of I. The predecessors to this paper are: Nonsolvable Finite Groups all of whose Local Subgroups are solvable, I, II, III: Bull A. M. S., 74 (1968), 383-437; Pacific J. Math., 33 (1970), 451-536; Pacific J. Math., 39 (1971), 483-534.

13. The case $2 \varepsilon \pi_{4}$; first reduction.

THEOREM 13.1.

(a) If $p \in \pi_{3}$, then $\mathscr{A}(p) \subseteq \mathscr{C}^{*}(\mathbb{S})$. ( $\mathscr{A}(p)$ is defined in Definition 2.10, and $\mathscr{L}^{*}(\mathbb{S})$ is defined in Definition 2.7.)

(b) If $p \in \pi_{3}, \mathfrak{P}$ is a $S_{p}$-subgroup of $\mathbb{S}$ and $\mathfrak{M}$ is the unique element of $\mathscr{C l} \mathscr{S}(\mathbb{S})$ which contains $\mathfrak{B}$, then

(i) $\mathfrak{P} \subseteq \mathfrak{M}^{\prime}$,

(ii) for each $G$ in $\left(\mathbb{S}\right.$ - $\mathfrak{M}, S_{p}$-subgroups of $\mathfrak{M} \cap \mathfrak{M}^{\text {G }}$ are of order 1 or $p$.

Proof. Theorem 10.7 implies (a); (b)(i) is a consequence of (a) and a standard transfer theorem; (b)(ii) can be established by imitating the proof of Theorem 0.25.6.

LEMMA 13.1. If $\mathfrak{X}$ is non identity 2-subgroup of (S), then $O_{2^{\prime}}(N(\mathfrak{X}))=1$.

Proof. Set $\mathfrak{R}=N(\mathfrak{X})$ and let $\mathfrak{I}$ be a $S_{2}$-subgroup of $\mathfrak{R}$. Suppose by way of contradiction that $O_{2^{\prime}}(\mathfrak{R}) \neq 1$. First, suppose $|\mathfrak{X}|=2$. Let $\mathfrak{P}$ be a minimal normal subgroup of $\mathfrak{N}$ of odd order. Thus, $\mathfrak{P}$ is a $p$-group for some odd prime $p$. Let $\mathfrak{F}$ be a maximal $2, p$-subgroup of $(\mathfrak{S})$ which contains $\mathfrak{T} \mathfrak{P}$. Let $\mathfrak{F}_{2}, \mathfrak{S}_{p}$ be a Sylow system of $\mathfrak{S}_{\mathfrak{C}}$ with $\mathfrak{I} \subseteq \mathfrak{S}_{2}, \mathfrak{P} \subseteq \mathfrak{S}_{p}$.

First, suppose $\boldsymbol{O}_{p}(\mathfrak{S})=1$. Let $\Re=\boldsymbol{O}_{2}(\mathfrak{S})$. By the $\mathfrak{P} \times \mathfrak{Z}$-lemma, 
$\mathfrak{P}$ is faithfully represented on $C_{\Re}(\mathfrak{X})$. But $\left[\mathfrak{P}, C_{\Re}(\mathfrak{X})\right] \subseteq[\mathfrak{P}, \mathfrak{R}] \subseteq \mathfrak{P}$, so $\mathfrak{P}$ centralizes $\boldsymbol{C}_{\mathfrak{R}}(\mathfrak{X})$.

We may assume that $O_{p}(\mathfrak{S}) \neq 1$. Suppose $O_{2}(\mathfrak{F}) \neq 1$. By Lemma 6.6 , we get $2 \notin \pi_{4}$, against our basic assumption of this section. So we may assume that $\boldsymbol{O}_{2}(\mathfrak{E})=1$. Since $\mathfrak{I}$ is a $S_{2}$-subgroup of $\mathfrak{R}$, Lemma 5.38 (a)(ii) implies that $\mathfrak{I}$ contains an element $\mathfrak{U}$ of $\mathscr{C}(2)$. By Lemma $6.1(b), \mathfrak{U}$ centralizes every element of $\mathfrak{U}\left(\mathfrak{H} ; 2^{\prime}\right)$. In particular, $\mathfrak{U}$ centralizes $\boldsymbol{O}_{p}(\mathfrak{S})$. This is also impossible, since $\boldsymbol{O}_{p}(\mathfrak{K})=\boldsymbol{F}(\mathfrak{K})$.

We may assume that $|\mathfrak{X}|>2$. Let $\mathfrak{Y}$ be a subgroup of order 2 in $\mathfrak{X} \cap \boldsymbol{Z}(\mathfrak{I})$. By the first part of the proof, $\boldsymbol{O}_{2^{\prime}}\left(\mathfrak{N}_{1}\right)=1$, where $\mathfrak{N}_{1}=\boldsymbol{N}(\mathfrak{Y})$. Hence, $\boldsymbol{O}_{2},(\mathfrak{R})$ is faithfully represented on $\boldsymbol{O}_{2}\left(\mathfrak{N}_{1}\right) \cap \boldsymbol{C}(\mathfrak{X})$, by the $\mathfrak{P} \times \mathfrak{Q}$-lemma. Since

$$
\left[\boldsymbol{O}_{2^{\prime}}(\mathfrak{N}), \boldsymbol{O}_{2}\left(\mathfrak{R}_{1}\right) \cap \boldsymbol{C}(\mathfrak{X})\right] \subseteq \boldsymbol{O}_{2}\left(\mathfrak{R}_{1}\right) \cap \boldsymbol{O}_{2^{\prime}}(\mathfrak{R})=1,
$$

we get $\boldsymbol{O}_{2^{\prime}}(\mathfrak{R})=1$.

THEOREM 13.2. Let $\mathfrak{M}$ be a solvable subgroup of $\mathbb{B S}$ and let $\mathfrak{M}_{2}$ be a $S_{2}$-subgroup of $\mathfrak{M}$. Then either $N\left(\mathfrak{M}_{2}\right) \nsubseteq \mathfrak{M}$ or $\mathfrak{M}_{2}$ contains an involution $I$ such that $C(I) \nsubseteq \mathfrak{M}$.

Proof. Suppose false. Then $\mathfrak{M}_{2}$ is a $S_{2}$-subgroup of (5) and $\mathfrak{M}$ contains the centralizer of each of its involutions. By Lemma 5.35, $\mathfrak{M}$ has just one class of involutions. By Lemma 5.40, $\mathfrak{M}$ has 2-length 1. Since $2 \in \pi_{4}$, we get $\mathfrak{M}_{2} \triangleleft \mathfrak{M}$. Thus, $\mathfrak{M}$ contains exactly $1 S_{2}$ subgroup of $\mathbb{B}$, and every involution of $\mathfrak{M}_{2}$ is central. This implies that $\mathfrak{M}_{2}$ is a T.I. set in $\mathbb{S}$. By a fundamental result of Suzuki [36], we have $(8) \cong U_{3}(q), S z(q), L_{2}(q)$, for some $q=2^{n}>2$. Since $U_{3}(q)$ is not an $N$-group, we get that $(B)$ satisfies the conclusions of the main theorem. The proof is complete.

The next lemma begins to pinpoint some of the difficulties of this section.

LEMma 13.2. Let $\mathfrak{I}$ be a $S_{2}$-subgroup of $\mathbb{B}$. Suppose $\mathfrak{T} \in \mathscr{L}^{*}(\mathbb{S})$. Let $\mathfrak{M}$ be the unique element of $\mathscr{M} \mathscr{S}(\mathbb{S})$ which contains $\mathfrak{T}$. Then there is a 2,3-subgroup $\mathfrak{F}$ of (B) such that

(a) $\mathfrak{S} \nsubseteq \mathfrak{M}$.

(b) $\mathfrak{S} \cap \mathfrak{M}$ contains a $S_{2}$-subgroup $\mathfrak{S}_{2}$ of $\mathfrak{S}$.

(c) $O_{3}(\mathfrak{S})=1$.

(d) $\mathfrak{S}_{2}$ contains an involution $I$ such that

(i) $C_{\mathfrak{s}_{2}}(I)$ is a $S_{2}$-subgroup of $\boldsymbol{C}_{\mathfrak{n}}(I)$.

(ii) $C_{\mathfrak{\xi}_{2}}(I)$ contains an element of $\mathscr{\mathscr { C }}(2)$.

(iii) $\boldsymbol{C}(I) \nsubseteq \mathfrak{M}$. 
(e) If $\mathfrak{S}_{2} \subseteq \mathfrak{M}_{0} \subseteq \mathfrak{M}$, and $\mathfrak{S}_{2}$ is not a $S_{2}$-subgroup of $\mathfrak{M}_{0}$, then $\mathfrak{M}_{0} \in \mathscr{C}^{*}(\mathbb{S})$.

Proof. Let $\mathscr{U}$ be the set of all $\mathfrak{u}$ in $\mathscr{C}(2)$ with $\mathfrak{U} \subseteq \mathfrak{M}$. Let $\mathscr{J}_{0}$ be the set of all involutions $I$ of $\mathfrak{M}$ such that

$(\alpha) C_{\mathfrak{M}}(I)$ contains an element of $\mathscr{Q}$.

( $\beta) C(I) \nsubseteq \mathfrak{M}$.

We first show that $\mathscr{T}_{0} \neq \varnothing$. Suppose false. Choose $\mathfrak{U}_{0} \in \mathscr{Z}_{\mathscr{L}}(\mathfrak{T})$, and set $\mathfrak{I}_{0}=C_{\mathfrak{z}}\left(\mathfrak{U}_{0}\right)$. Since $\mathfrak{H}_{0} \in \mathscr{U}$, it follows that $C(I) \subseteq \mathfrak{M}$ for all $I \in \mathfrak{I}_{0}^{\sharp}$. On the other hand, since $\mathfrak{I} \in \mathscr{C}^{*}(\mathbb{S})$, we have $N(\mathfrak{T}) \subseteq \mathfrak{M}$, so by Theorem 13.2, there is an involution $J_{0}$ of $\mathfrak{M}$ such that $C\left(J_{0}\right) \nsubseteq \mathfrak{M}$. Let $\mathfrak{M}_{2}$ be a $S_{2}$-subgroup of $C_{\mathfrak{n}}\left(J_{0}\right)$. Choose $M$ in $\mathfrak{M}$ such that $\mathfrak{M}_{2} \subseteq \mathfrak{I}$, and set $J=J_{0}^{M}$. Thus, $\widetilde{\mathfrak{M}}_{2}=\mathfrak{M}_{2}^{M}$ is a $S_{2}$-subgroup of $C_{\mathfrak{M}}(J)$ and $C(J) \nsubseteq$ $\mathfrak{M}$. Hence, $J \notin \mathfrak{I}_{0}$ and $\widetilde{\mathfrak{M}}_{2}$ contains no element of $\mathscr{\mathscr { C }}$, since $J \notin \mathscr{F}_{0}$. By Lemma 5.38 (a)(ii), $C(J)$ contains an element of $\mathscr{L}(2)$. Hence, $\widetilde{\mathfrak{M}}_{2}$ is not a $S_{2}$-subgroup of $C(J)$. Let $\mathfrak{M}_{3}$ be a 2-subgroup of $C(J)$ with $\left|\mathfrak{M}_{3}: \widetilde{\mathfrak{M}}_{2}\right|=2$. Choose $X \in \mathfrak{M}_{3}-\widetilde{\mathfrak{M}}_{2}$, so that $X \notin \mathfrak{M}$. Hence, $C(X)$ contains no element of $\mathfrak{T}_{0}^{\sharp}$. On the other hand, $\boldsymbol{D}\left(\widetilde{\mathfrak{M}}_{2}\right) \subseteq \mathfrak{I}_{0}$, so $\boldsymbol{D}\left(\widetilde{\mathfrak{M}}_{2}\right)=1$. Since $\widetilde{\mathfrak{M}}_{2} \cap \mathfrak{I}_{0}$ is of index 2 in $\widetilde{\mathfrak{M}}_{2}$, it follows that $C(X) \cap \widetilde{\mathfrak{M}}_{2}=\langle J\rangle$. Hence, $\widetilde{\mathfrak{M}}_{2}$ is a four-group. This implies that $\mathfrak{I}$ is of maximal class, against $2 \in \pi_{4}$. We conclude that $\mathscr{F}_{0} \neq \varnothing$.

Let $\mathscr{T}$ be the set of all 2-subgroups $\mathfrak{I}_{1}$ of $\mathfrak{M}$ with the following properties:

(1) $\mathfrak{I}_{1} \notin \mathscr{C l}^{*}(\mathbb{B})$.

(2) There is at least one involution $T$ of $\mathscr{F}_{0}$ such that $\mathfrak{I}_{1}$ contains a $S_{2}$-subgroup of $C_{\mathfrak{m}}(T)$.

We argue that $\mathscr{T} \neq \varnothing$. Namely, choose $I \in \mathscr{I}_{0}$, and let $\mathfrak{I}_{1}$ be a $S_{2}$-subgroup of $C_{\mathfrak{m}}(I)$. Since $C(I) \nsubseteq \mathbb{M}$, we get that $\mathfrak{I}_{1} \in \mathscr{T}$.

Let $\mathfrak{S}_{2}$ be an element of $\mathscr{T}$ of maximal order. Since $\mathfrak{I} \in \mathscr{C}^{*}(\mathbb{S})$, it follows that $\mathfrak{S}_{2}$ is not a $S_{2}$-subgroup of $\mathfrak{M}$. By maximality of $\mathfrak{F}_{2}$, (e) holds. Let $\mathscr{S}=\left\{\mathfrak{S} \mid \mathscr{S}_{2} \subseteq \mathfrak{S} \in \mathscr{S} \mathscr{O} \mathscr{L}(\mathbb{S}), \mathfrak{S} \nsubseteq \mathfrak{M}\right\}$. By definition $\mathscr{T}$, we have $\mathscr{S} \neq \varnothing$. If $\subseteq \in \mathscr{S}$, the maximality of $\mathfrak{S}_{2}$ guarantees that $\mathfrak{S}_{2}$ is a $S_{2}$-subgroup of $\mathfrak{S}$. Let $\pi=\{p \mid p$ is an odd prime, $\mathscr{S}$ contains a $2, p$-group\}. Thus, $\pi \neq \varnothing$. Choose $p \in \pi$ and let $\mathfrak{F}$ be a $2, p$-subgroup of $B 5$ which is contained in $\mathscr{S}$, and is maximal with this property. Thus, $\mathfrak{S}_{\mathfrak{Z}}$ is a maximal $2, p$-subgroup of (5) which is not a $S_{2, p}$-subgroup of (B).

By definition of $\mathscr{T}$, there is $T$ in $\mathscr{F}_{0}$ such that $C_{\mathfrak{F}_{2}}(T)$ is a $S_{2^{-}}$ subgroup of $C_{\mathfrak{m}}(T)$. By definition of $\mathscr{F}_{0}$, we get that $\mathfrak{S}_{2}$ contains an element $\mathfrak{l}$ of $\mathscr{U}$. Thus, (d) holds.

By Lemma 6.6, either $\boldsymbol{O}_{2}(\mathfrak{S})=1$, or $\boldsymbol{O}_{p}(\mathfrak{S})=1$. By Lemma $6.1(\mathrm{~b})$, $\mathfrak{U}$ centralizes $\boldsymbol{O}_{p}(\mathfrak{S})$, so $\boldsymbol{O}_{2}(\mathfrak{Y}) \neq 1$. We thus get $\boldsymbol{O}_{p}(\mathfrak{S})=1$, so if $p=3$, 
we are done. Suppose $p \geqq 5$. Then by [43], we have

$$
\mathfrak{S}=C_{\mathfrak{S}}\left(Z\left(\mathfrak{S}_{2}\right)\right) N_{\mathfrak{F}}\left(J\left(\mathfrak{S}_{2}\right)\right) \text {. }
$$

Since $\left|N_{\mathfrak{M}}(\Re)\right|_{2}>\left|\mathscr{S}_{2}\right|$ for $\Re=Z\left(\mathscr{S}_{2}\right)$ and for $\Re=J\left(\mathscr{S}_{2}\right)$, the maximality of $\mathfrak{S}_{2}$ forces $\mathfrak{S} \subseteq \mathfrak{M}$, against our construction. The proof is complete.

For each odd prime $p$, let $e(p)$ be the largest integer $n$ such that $U(\& ; 2)$ is non trivial for some elementary subgroup \& of \&s of order $p^{n}$. Let

$$
e=e(G)=\max \{e(p)\},
$$

where $p$ ranges over all odd primes. Since (S) is simple, there is a 2-subgroup $\mathfrak{A}$ of $\mathbb{B S}$ such that $\boldsymbol{A}_{\mathscr{\Theta}}(\mathfrak{Z})$ is not a 2-group, by Theorem 14.4.7 of [21]. Hence, $e \geqq 1$.

Hypothesis 13.1. $e \geqq 3$.

LEMmA 13.3 through 13.38 are proved under Hypothesis 13.1.

We use the following notation: $r_{0}$ denotes an odd prime such that for some elementary subgroup $\Re_{0}$ of $B$ of order $r_{0}^{3}, h\left(\Re_{0} ; 2\right)$ is non trivial; $\mathfrak{I}_{0}$ is a maximal element of $\boldsymbol{U}\left(\Re_{0} ; 2\right)$.

\section{Lemma 13.3. $\mathscr{A}\left(r_{0}\right) \subseteq \mathscr{C}^{*}(G)$.}

Proof. If $r_{0} \in \pi_{3}$, we may apply Theorem 13.1. Thus, we may assume that $r_{0} \notin \pi_{3}$. Since $\Re_{0}$ is elementary of order $r_{0}^{3}$, we conclude that $r_{0} \in \pi_{4}$. If $r_{0} \geqq 5$, we may apply Lemma 10.6. Thus, we may assume that $r_{0}=3$.

By Lemma 13.1, $\Re_{0}$ acts faithfully on $\boldsymbol{O}_{2}\left(N\left(\mathfrak{I}_{0}\right)\right)$. Thus, $\boldsymbol{O}_{2}\left(N\left(\mathfrak{I}_{0}\right)\right)$ contains a non cyclic abelian group of order 8 . Since $\Re_{0}$ is elementary of order $3^{3}$, we get $2 \sim 3$. If the center of a $S_{3}$-subgroup of $(S)$ is non cyclic, then $B=E_{2}(3)$, by Theorem 8.1. If the center of a $S_{3}$ subgroup of $\mathbb{B S}$ is cyclic, then $\mathbb{B S} \cong S_{4}(3)$, by Theorem 9.1 . Since $\mathbb{B}$ is an $N$-group, both of these possibilities are excluded. The proof is complete.

We now set $\mathfrak{M}=M\left(\Re_{0}\right)$. Let $\sigma=\left\{p \mid p \in \pi_{3} \cup \pi_{4}, \mathfrak{M}\right.$ contains a $S_{p}$-subgroup of \&S\}. Thus, $r_{0} \in \sigma$, and if $p \in \pi_{3}$, then $p \in \sigma$ if and only if $\mathfrak{M}$ contains an element of $\mathscr{A}(p)$. Choose $p$ in $\sigma$ and let $\mathfrak{P}$ be a $S_{p}$-subgroup of $\mathfrak{M}$ permutable with the $S_{2}$-subgroup $\mathfrak{I}$ of $\mathfrak{M}$.

LEMMA 13.4. $\quad O_{p}(\mathfrak{P T})=1$.

Proof. If $\mathfrak{I}$ is a $S_{2}$-subgroup of $\mathbb{B}$, we are done, since $2 \in \pi_{4}$. We may assume that $\mathfrak{I}$ is not a $S_{2}$-subgroup of $\mathbb{B}$. 
Since $\mathfrak{F} \in \mathscr{C}^{*}(\mathbb{S}), \mathfrak{P T}$ is a maximal $2, p$-subgroup of $\mathbb{S}$. Suppose $O_{p}(\mathfrak{P T}) \neq 1, O_{2}(\mathfrak{P T}) \neq 1$. Then Lemma 6.6 (iii) yields a contradiction. Thus, proceeding by way of contradiction, we may assume that $O_{p}(\mathfrak{B T}) \neq 1, O_{2}(\mathfrak{P T})=1$. Since $O_{2}(\mathfrak{P T})=1$, it follows from Lemma 6.1 that $\mathfrak{I}$ contains no element of $\mathscr{C}(2)$. We will show that this is false. Since $\Re_{0} \in \mathscr{l}^{*}(\mathfrak{S})$, it follows that $N\left(\mathfrak{I}_{0}\right) \subseteq \mathfrak{M}$, and that $N\left(\mathfrak{I}_{1}\right) \subseteq \mathfrak{M}$ for every non identity characteristic subgroup $\mathfrak{I}_{1}$ of $\mathfrak{I}_{0}$. Since $\mathfrak{M}$ contains no element of $\mathscr{C}(2)$, it follows that $\mathfrak{I}_{0}$ is elementary.

Since $\Re_{0}$ is elementary of order $r_{0}^{3}$, we can choose a subgroup $\Re_{1}$ of $\Re_{0}$ of order $r_{0}^{2}$ such that $\mathfrak{I}_{0} \cap \boldsymbol{C}\left(\Re_{1}\right) \neq 1$. Let $I$ be an involution in $\mathfrak{I}_{0} \cap C\left(\Re_{1}\right)$. By Lemma 5.38 (a)(ii), $C(I)$ contains an element of $\mathscr{L}$ (2). Since $\Re_{1} \leqq C(I)$ and since $\Re_{1} \in \mathscr{A}\left(r_{0}\right)$, it follows from Lemma 13.2 that $C(I) \subseteq \mathfrak{M}$. This contradiction completes the proof.

\section{LEMMA 13.5 .}

(a) If $\mathrm{BS}_{2}$ is a $S_{2}$-subgroup of (5) which contains $\mathfrak{I}$, then $\mathfrak{I}$ contains every element of $\mathscr{Q} 6\left(\mathbb{S}_{2}\right)$.

(b) $O_{2}(\mathfrak{M}) \neq 1$.

(c) $O_{2^{\prime}}(\mathfrak{M})=1$.

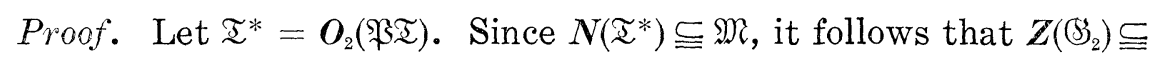
T. Since $\boldsymbol{O}_{p}(\mathfrak{P T})=1$, it follows that $Z\left(\mathfrak{S}_{2}\right) \subseteq \mathfrak{I}^{*}$. Hence, $N\left(\mathfrak{I}^{*}\right)$ contains every element of $\mathscr{C}\left(\mathrm{BS}_{2}\right)$, proving (a). Lemma 6.1 together with (a) yield (b).

Since $\mathbb{B S}$ is an $N$-group, $\mathfrak{M} \in \mathscr{C l} \mathscr{S}(\mathbb{S})$, and $O_{2}(\mathfrak{M}) \neq 1$, it follows that $\mathfrak{M}=\boldsymbol{N}\left(\boldsymbol{O}_{2}(\mathfrak{M})\right)$. Thus, Lemma 13.1 implies that (c) holds.

In Lemmas 13.6 through 13.38, we use the following notation: $\mathfrak{I}$ is a $S_{2}$-subgroup of $\mathfrak{M}, \mathfrak{B}=\Omega_{1}\left(\boldsymbol{R}_{2}(\mathfrak{M})\right), \mathfrak{B}^{*}=V\left(\operatorname{ccl}_{\mathfrak{\Theta}}(\mathfrak{B}) ; \mathfrak{I}\right)$. Also, $\sigma$ has its previous meaning. We also introduce the set $\mathscr{F}$ of all involutions $I$ of $\mathfrak{M}$ such that $C_{\mathfrak{M}}(I) \in \mathscr{M}^{*}(\mathbb{S})$. This set plays an important role in much of the following discussion.

By Lemma 5.9 (iii), $\mathfrak{B}$ is 2-reducible in $\mathfrak{M}$.

\section{LEMMA 13.6.}

(a) If $|\mathfrak{B}|>2$, then $C\left(\mathfrak{B}_{0}\right) \subseteq \mathfrak{M}$ for every hyperplane $\mathfrak{B}_{0}$ of $\mathfrak{B}$.

(b) One of the following holds:

(i) $|\mathfrak{B}|=2$.

(ii) If $\mathfrak{B}_{0}$ is a subgroup of index 2 in $\mathfrak{B}$ and $I$ is an involution of $\mathfrak{M}$ such that $C_{\mathfrak{B}}(I)=\mathfrak{B}_{0}$, then $[\mathfrak{B}, I]=\langle J\rangle$ is of order 2, and $J \in \mathscr{F}$.

(c) If $|\mathfrak{B}|>2$, then $\mathfrak{B}$ contains a non cyclic subgroup $\mathfrak{X}$ such that $\mathfrak{X}^{\ddagger} \subseteq \mathscr{F}$.

Proof. Let $\mathfrak{F}$ be an elementary subgroup of $\mathfrak{M}$ of order $p^{3}, p \in \sigma$. 
Assume that $|\mathfrak{B}|>2$. Since $\mathfrak{B} \triangleleft \mathfrak{M}$, there is a subgroup $\mathfrak{F}_{0}$ of $\mathfrak{E}$ of order $p^{2}$ such that $C\left(\mathscr{F}_{0}\right) \cap \mathfrak{B}=\mathfrak{X}$ is non cyclic. This proves (c), since $\mathfrak{F}_{0} \in \mathscr{C}^{*}(\mathbb{S})$. Let $\mathfrak{B}_{0}$ be of index 2 in $\mathfrak{B}$. Then $\mathfrak{B}_{0} \cap \mathfrak{X} \neq 1$, and $\boldsymbol{C}\left(\mathfrak{B}_{0}\right) \subseteq \boldsymbol{C}\left(\mathfrak{B}_{0} \cap \mathfrak{X}\right)$, proving (a).

In proving (b), we may assume that $|\mathfrak{B}|>2$. Let $\mathfrak{B}$ play the role of $\sqrt{5}$ in Lemma 5.21. We conclude that $C_{\mathfrak{n}}([\mathfrak{B}, I])$ contains an element of $\mathscr{A}(q)$ for some $q \in \sigma$. This yields (b).

\section{LEMMA 13.7. $\mathfrak{B} \subseteq Z\left(\mathfrak{B}^{*}\right)$.}

Proof. Suppose false. Thus, $|\mathfrak{B}|>2$. Choose $G$ in (8) such that $\mathfrak{X}=\mathfrak{B}^{G} \leqq \mathfrak{I}, \mathfrak{X} \nsubseteq C(\mathfrak{B})$, and define $d$ by $2^{d}=|\mathfrak{X}: \mathfrak{X} \cap C(\mathfrak{B})|$, so that $d \geqq 1$. Let $\overline{\mathfrak{M}}=\mathfrak{M} / \boldsymbol{C}(\mathfrak{B})$, and let $\overline{\mathfrak{X}}=\mathfrak{X} \boldsymbol{C}(\mathfrak{B}) / C(\mathfrak{B}) \cong \mathfrak{X} / \mathfrak{X} \cap \boldsymbol{C}(\mathfrak{B})$. Since $\mathfrak{B}$ is 2reducible in $\mathfrak{M}$, Lemma 5.34 implies that $\overline{\mathfrak{M}}$ contains a subgroup $\mathfrak{U}=\mathfrak{U}_{1} \times \cdots \times \mathfrak{U}_{d}$, where $\mathfrak{U}_{i}$ is of odd prime order $p_{i}$, admits $\mathfrak{X}$, and such that $\overline{\mathfrak{X}}$ is faithfully represented on $\mathfrak{X}$.

Let $\mathfrak{B}_{i}=\left[\mathfrak{B}, \mathfrak{A}_{i}\right], \overline{\mathfrak{X}}_{i}=\overline{\mathfrak{X}} \cap \boldsymbol{C}\left(\mathfrak{A}_{i}\right)$, and let $\mathfrak{X}_{i}$ be the inverse image in $\mathfrak{X}$ of $\overline{\mathfrak{X}}_{i}$. Thus, $\left|\mathfrak{X}: \mathfrak{X}_{i}\right|=\left|\overline{\mathfrak{X}}_{:} \overline{\mathfrak{X}}_{i}\right|=2,1 \leqq i \leqq d$.

By construction, we see that $\mathfrak{A}_{i}$ is faithfully represented on $\mathfrak{B}_{i}$ and that $\mathfrak{B}_{i}$ admits $\mathfrak{X} \overline{\mathfrak{X}}$. Let $\mathfrak{B}_{i}^{*}=\mathfrak{B}_{i} \cap \boldsymbol{C}\left(\mathfrak{X}_{i}\right)$. By Lemma 3.7 of [20], $\mathfrak{N}_{i}$ is faithfully represented on $\mathfrak{B}_{i}^{*}$, so in particular, $\mathfrak{X}$ does not centralize $\mathfrak{B}_{i}^{*}$. Choose $V_{i}$ in $\mathfrak{B}_{i}^{*}-\mathfrak{B}_{i}^{*} \cap \boldsymbol{C}(\mathfrak{X})$. By Lemma 13.6(a) applied to $\mathfrak{M}^{G}$, we get $C\left(\mathfrak{X}_{i}\right) \subseteq \mathfrak{M}^{G}$. Hence, $V_{i} \in \mathfrak{M}^{G}$. By Lemma 13.6(b) applied to $\mathfrak{M}^{G}$, we get $\boldsymbol{C}\left(\left[\mathfrak{X}, V_{i}\right]\right)=\boldsymbol{C}_{\mathfrak{M}}\left(\left[\mathfrak{X}, V_{i}\right]\right) \in \mathscr{C}^{*}(\mathbb{S})$. Choose $W_{i}$ in $\left[\mathfrak{X}, V_{i}\right]^{\sharp}$. Then $C\left(W_{i}\right) \subseteq \mathfrak{M}^{a}$. Since $W_{i} \in \mathfrak{B}$ and $\mathfrak{B}$ is abelian, we get $\mathfrak{B} \subseteq \mathfrak{M}^{a}$. Hence, $[\mathfrak{B}, \mathfrak{X}] \subseteq \mathfrak{X}$, since $\mathfrak{X} \triangleleft \mathfrak{M}^{a}$. In particular, $\left[\mathfrak{B}_{i}, \overline{\mathfrak{X}}_{i}\right]$ is centralized by $\overline{\mathfrak{X}}$, so $\left[\mathfrak{B}_{i}, \overline{\mathfrak{X}}_{i}\right]$ is centralized by $\mathfrak{N}_{i}$. As $\mathfrak{X}_{i}$ has no fixed points on $\mathfrak{B}_{i}^{*}$, we conclude that $\left[\mathfrak{B}_{i}, \mathfrak{X}_{i}\right]=1$. Since $\mathfrak{B}_{i}$ admits $\mathfrak{A}$, we conclude that $\left\langle\mathfrak{U}_{j} \mid j \neq i\right\rangle=\mathfrak{U}^{i}$ centralizes $\mathfrak{B}_{i}$, and in particular centralizes $\mathfrak{W}_{i}$. Now $\mathfrak{U}^{i}=\mathfrak{L} / C(\mathfrak{B})$ for a suitable subgroup $\mathbb{Z}$ of $\mathfrak{M}$, so $\mathbb{Z}$ centralizes $W_{i}$. Since $C\left(W_{i}\right) \subseteq \mathfrak{M}^{a}$, we conclude that $[\mathbb{R}, \mathfrak{X}] \subseteq\left[\mathfrak{M}^{a}, \mathfrak{X}\right] \subseteq \mathfrak{X}$. Thus, $[\Omega, \mathfrak{X}] \subseteq C(\mathfrak{B})$. Hence, $\mathfrak{U}^{i}$ is centralized by $\mathfrak{X}$. By construction, we conclude that $d=1$, that is, $|\mathfrak{X}: \mathfrak{X} \cap C(\mathfrak{B})|=2$.

Since $\mathfrak{B} \subseteq \mathfrak{M}^{a}$ and $[\mathfrak{B}, \mathfrak{X}] \neq 1$, we conclude by symmetry that $|\mathfrak{B}: \mathfrak{B} \cap C(\mathfrak{X})|=2$. Choose $V$ in $\mathfrak{B}-C(\mathfrak{X}) \cap \mathfrak{B}, X$ in $\mathfrak{X}-C(\mathfrak{B}) \cap \mathfrak{X}$. Thus, $[V, X]$ is an involution and is a generator for $[\mathfrak{B}, X]=[V, \mathfrak{X}]$. We may apply Lemma 13.6 (b) twice to conclude that $\mathfrak{M}=\mathfrak{M}^{a}$. Thus, $\mathfrak{B}=\mathfrak{X}=\mathfrak{B}^{G}$, which is absurd. The proof is complete.

LEMMA 13.8.

(a ) $\mathfrak{M}=C(\mathfrak{B}) \cdot N_{\mathfrak{M}}\left(\mathfrak{B}^{*}\right)$.

(b) One of the following holds:

(i) For each $V$ in $\mathfrak{B}^{*}, C_{\mathfrak{m}}(V) \in \mathscr{C}^{*}(\mathbb{S})$, that is $\mathfrak{B}^{*} \leqq \mathscr{Y}$.

(ii) $N_{\mathfrak{m}}\left(\mathfrak{S}^{*}\right) \in \mathscr{L}^{*}(\mathbb{S})$. 
(c) $\mathfrak{I}$ is a $S_{2}$-subgroup of $(8)$.

Proof. By Lemma 13.7, $\mathfrak{S}^{*} \sqsubseteq C(\mathfrak{B})$. Since $\mathfrak{Z}^{*}$ is weakly closed in $\mathfrak{I} \cap C(\mathfrak{B})$, (a) holds.

Let $\mathfrak{P}$ be a $S_{p}$-subgroup of $\mathfrak{M}, p \in \sigma$. Suppose $\mathfrak{P} \cap C(\mathfrak{B})$ is non cyclic. Since $\mathfrak{P} \cap \boldsymbol{C}(\mathfrak{B}) \triangleleft \mathfrak{P}$, it follows that $\mathfrak{P} \cap \boldsymbol{C}(\mathfrak{B})$ contains an element of $\mathscr{A}(p)$. Thus, in this case, (b)(i) holds. Suppose $\mathfrak{P} \cap \boldsymbol{C}(\mathfrak{B})$ is cyclic. In this case, the argument of Theorem 0.24 .9 shows that $\boldsymbol{N}_{\mathfrak{*}}\left(\mathfrak{B}^{*}\right)$ contains an element of $\mathscr{A}(p)$, so that (b) (ii) holds.

Let $\mathbb{S}_{2}$ be a $S_{2}$-subgroup of $\left(\mathcal{S}\right.$ which contains $\mathfrak{T}$. Since $\boldsymbol{O}_{2},(\mathfrak{M})=1$, it follows that $\Omega_{1}\left(\mathbb{Z}\left(\mathcal{S}_{2}\right)\right) \subseteq \mathfrak{B}$, by Lemma 5.40. If (b)(i) holds, then we may choose $V$ in $\Omega_{1}\left(Z\left(\mathcal{S}_{2}\right)\right)$ and conclude that $\mathfrak{I}=\mathbb{S}_{2}$. If (b)(ii) holds, then $N\left(\mathfrak{B}^{*}\right) \subseteq \mathfrak{M}$, so that $\mathfrak{I}=\mathfrak{G}_{2}$, since $\mathfrak{B}^{*}$ is weakly closed in T. The proof is complete.

\section{LEMMA 13.9. $N(\mathfrak{I}) \subseteq \mathfrak{M}$.}

Proof. Choose $N$ in $N(\mathfrak{I})$. First, suppose (b)(i) of Lemma 13.8

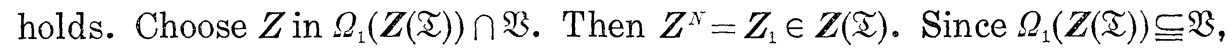
we conclude that $Z_{1} \in \mathfrak{B}$. Since $(\mathrm{b})(\mathrm{i})$ holds, $C_{\mathfrak{M}}(Z)=\boldsymbol{C}_{\mathfrak{G}}(Z) \in \mathscr{Z l}^{*}(\mathrm{~S})$, $C_{\mathfrak{M}}\left(Z_{1}\right)=C_{\mathfrak{G}}\left(Z_{1}\right) \in \mathscr{l}^{*}(\mathrm{~S})$. But $C\left(Z_{1}\right)=C(Z)^{N}$, so $\mathfrak{M}=\mathfrak{M}^{N}, N \in \mathfrak{M}$. Suppose (b)(ii) holds. Then $N\left(\mathfrak{B}^{*}\right) \subseteq \mathfrak{M}$. Since $N$ normalizes $\mathfrak{B}^{*}$, the proof is complete.

LEMMA 13.10. Suppose $\mathfrak{U}$ is a four-subgroup of $\mathfrak{M}, \mathfrak{N} \cap \boldsymbol{C}(\mathfrak{B})=1$ and $C(\mathfrak{Y}) \cap \mathfrak{B}$ contains a subgroup $\tilde{\mathfrak{B}}$ with $|\mathfrak{B}: \tilde{\mathfrak{B}}|=4$. Then for each element $V$ of $\mathfrak{B}-\tilde{\mathfrak{B}}$, there is an element $A$ in $\mathfrak{X}$ such that $C([V, A]) \subseteq \mathfrak{M}$.

Proof. For each subset $\subseteq$ of $\mathfrak{M}$, let $\bar{\complement}=\subseteq C(\mathfrak{B}) / C(\mathfrak{B})$. Thus, $\mathfrak{U} \cong \overline{\mathfrak{A}}$. By Lemma 5.34, we may choose a subgroup $\mathfrak{B}=\mathfrak{B}_{0} \times \mathfrak{B}_{1}$ of $\mathfrak{M}$ such that $\mathfrak{B}_{i}$ is of prime order $p_{i}, \mathfrak{B}_{i}$ admits $\overline{\mathfrak{A}}, i=0,1$, and such that $\overline{\mathfrak{A}}$ is faithfully represented on $\mathfrak{B}$. Let $\mathfrak{U}_{i}$ be the subgroup of $\mathfrak{N}$ such that $\overline{\mathfrak{A}}_{i}=C\left(\mathfrak{B}_{i}\right) \cap \overline{\mathfrak{A}}$. Thus, $\left|\mathfrak{U}_{i}\right|=2, i=0,1$, and $\mathfrak{U}=\mathfrak{U}_{0} \times \mathfrak{U}_{1}$.

Let $\mathfrak{B}_{i}=\left[\mathfrak{B}, \mathfrak{B}_{i}\right]$; thus, $\mathfrak{B}_{i}$ has no fixed points on $\mathfrak{B}_{i}^{*}$, and the dihedral group $\mathfrak{B}_{i} \mathfrak{N}_{j}$ is faithfully represented on $\mathfrak{B}_{i}, i, j=0,1, i \neq j$. Thus, $\left|\mathfrak{B}_{i}\right|=2^{2 d_{i}}$, where $d_{i}$ is an integer. Also, $\left|\boldsymbol{C}\left(\mathfrak{Y}_{j}\right) \cap \mathfrak{B}_{i}\right|=2^{d_{i}}$. Suppose $d_{i} \geqq 2$. Since $\mathfrak{A}$ centralizes $\tilde{\mathfrak{B}}$, it follows that $d_{i}=2$, and that $\boldsymbol{C}\left(\mathfrak{U}_{j}\right) \cap \mathfrak{B}_{i}=\tilde{\mathfrak{B}} \cap \mathfrak{B}_{i}$. Since $\mathfrak{B}_{i} \cap \boldsymbol{C}\left(\mathfrak{N}_{i}\right)$ admits $\mathfrak{B}_{i} \mathfrak{N}_{j}$, and since $\tilde{\mathfrak{B}} \cap \mathfrak{B}_{i}$ does not admit $\mathfrak{B}_{i} \mathfrak{Z}_{j}$, it follows that $\mathfrak{B}_{i} \cap \boldsymbol{C}\left(\mathfrak{U}_{i}\right)$ properly contains $\tilde{\mathfrak{B}} \cap \mathfrak{B}_{i}$. The only possibility is that $\mathfrak{N}_{i}$ centralizes $\mathfrak{B}_{i}$. But since $\tilde{\mathfrak{B}}$ is of index 4 in $\mathfrak{B}$, we have $\mathfrak{B}=\mathfrak{V}_{i} \mathfrak{B}$, so $\mathfrak{N}_{i} \subseteq C(\mathfrak{B})$, against the hypothesis that $\mathfrak{A} \cap \boldsymbol{C}(\mathfrak{B})=1$. We conclude that $d_{i}=1$.

Since $\left|\mathfrak{B}_{i}\right|=4$, it follows that $\mathfrak{B}=\mathfrak{B}_{0} \times \mathfrak{B}_{1} \times \mathfrak{X}$, where $\mathfrak{X}=C_{\mathfrak{B}}(\mathfrak{B})$. Furthermore, since $\mathfrak{B}$ is of index 4 in $\mathfrak{B}$, it follows that $\tilde{\mathfrak{B}}=\tilde{\mathfrak{B}}_{0} \times$ 
$\tilde{\mathfrak{B}}_{1} \times \mathfrak{X}$, where $\tilde{\mathfrak{B}}_{i}=\tilde{\mathfrak{B}} \cap \mathfrak{B}_{i}$ is of order 2. Let $\mathfrak{N}_{i}=\left\langle A_{i}\right\rangle$. Then $\mathfrak{B} \cap \boldsymbol{C}\left(A_{i}\right)=\mathfrak{X} \times \mathfrak{B}_{i} \times \tilde{\mathfrak{B}}_{j}$ is of index 2 in $\mathfrak{B}$, and so by Lemma 5.21, we conclude that $C_{\mathfrak{m}}\left(\left[A_{i}, \mathfrak{B}\right]\right)$ contains an element of $\mathscr{A}\left(q_{i}\right)$ for some $q_{i}$ in $\sigma$. Suppose now that $V \in \mathfrak{B}-\tilde{\mathfrak{B}}$. Then $V=V_{0} V_{1} X$, where $V_{i} \in \mathfrak{B}_{i}, X \in \mathfrak{X}$. Since $V \notin \tilde{\mathfrak{B}}$, there is an index $i$ such that $V_{i} \notin \tilde{\mathfrak{B}}_{i}$. Hence, $\left[A_{j}, V_{i}\right]=\left[A_{j}, V\right]$, where $j \neq i$, so that $C\left(\left[A_{j}, V_{i}\right]\right) \subseteq \mathfrak{M}$, as required.

Lemma 13.11. One of the following holds:

(a) $|\mathfrak{B}| \leqq 4$.

(b ) $\quad \boldsymbol{C}\left(\mathfrak{B}_{0}\right) \subseteq \mathfrak{M}$ for every subgroup $\mathfrak{B}_{0}$ of $\mathfrak{B}$ of index 4 .

Proof. We may assume that $|\mathfrak{B}| \geqq 8$. Let $\&$ be an elementary subgroup of $\mathfrak{M}$ of order $p^{3}, p \in \sigma$, and let $\mathfrak{B}_{0}$ be a subgroup of index 4 in $\mathfrak{B}$ such that $\boldsymbol{C}\left(\mathfrak{B}_{0}\right) \nsubseteq \mathfrak{M}$. Hence, $\mathfrak{B}_{0} \cap \mathscr{J}=\varnothing$.

Let $\mathfrak{B}=\mathfrak{B}_{1} \times \cdots \times \mathfrak{B}_{f}$, where each $\mathfrak{B}_{i}$ is an irreducible 5 -group. Let $\mathfrak{F}_{i}=\mathbb{F} \cap \boldsymbol{C}\left(\mathfrak{B}_{i}\right)$. Since $\mathfrak{F}_{i}$ is non cyclic, it follows that $\mathfrak{B}_{i}^{\sharp} \cong \mathscr{J}$. Hence, $\mathfrak{B}_{i} \cap \mathfrak{B}_{0}=1,1 \leqq i \leqq f$. Since $\left|\mathfrak{B}_{:} \mathfrak{B}_{0}\right|=4$, it follows that $\left|\mathfrak{B}_{i}\right| \leqq 4,1 \leqq i \leqq f$.

Clearly, $\&$ does not centralize $\mathfrak{B}$. Suppose $\left|\mathfrak{B}_{i}\right|=2$ for some $i$. Choose $j$ so that $\left|\mathfrak{B}_{j}\right|>2$. Then $\left(\mathfrak{B}_{i} \mathfrak{B}_{j}\right)^{\sharp} \subseteq \mathscr{F}$, against $\mathfrak{B}_{0} \cap \mathscr{J}=\varnothing$. We conclude that $\mathfrak{B} \cap \boldsymbol{C}(\mathfrak{F})=1$, and that $\mathbb{F}$ is of order $3^{3}$. Let $\mathfrak{A}=$ $\left(\mathfrak{B}_{1} \mathfrak{B}_{2}\right) \cap \mathfrak{B}_{0}$. Thus, $|\mathfrak{A}| \geqq 4$, since $\left|\mathfrak{B}_{:} \mathfrak{B}_{0}\right|=4$. Since $\mathfrak{U} \cap \mathfrak{B}_{i}=1, i=$ 1,2 , it follows that $|\mathfrak{A}|=4$. Let $\mathfrak{F}^{*}$ be a complement to $\mathfrak{F}_{1} \cap \mathfrak{F}_{2}$ in ․ Thus, $\left|F^{*}\right|=3^{2}$ and $\mathfrak{F}^{*}$ is faithfully represented on $\mathfrak{B}_{1} \mathfrak{B}_{2}$. By Lemma 5.31, $\mathfrak{F}^{*}$ has a subgroup $\mathfrak{F}_{1}^{*}$ of order 3 which normalizes $\mathfrak{A}$. Thus, $\left\langle\mathfrak{F}_{1} \cap \mathfrak{F}_{2}, \mathfrak{F}_{1}^{*}\right\rangle \subseteq N(\mathfrak{Q})$. Since $\left\langle\mathfrak{F}_{1} \cap \mathfrak{F}_{2}, \mathfrak{F}_{1}^{*}\right\rangle \in \mathscr{A}(3)$ it follows that $\mathfrak{R}(\mathfrak{U}) \subseteq \mathfrak{M}$. Since $\boldsymbol{C}\left(\mathfrak{B}_{0}\right) \subseteq C(\mathfrak{U}) \subseteq N(\mathfrak{U}) \subseteq \mathfrak{M}$, we have the desired contradiction. The proof is complete.

LEMma 13.12. Suppose (5) is an elementary subgroup of $\boldsymbol{A}_{\oplus}(\mathfrak{B})$ of order 8. Then $\mathfrak{B}$ does not contain any subgroup $\mathfrak{X}$ of index 2 such that [X, (5)] is of order 2.

Proof. In accordance with Lemma 5.34, choose $\mathfrak{B} \subseteq \boldsymbol{A}_{\circledast}(\mathfrak{B})$ such that $\mathfrak{B}=\mathfrak{B}_{1} \times \mathfrak{B}_{2} \times \mathfrak{B}_{3}$, where $\mathfrak{B}_{i}$ is of prime order $p_{i}, \mathfrak{B}_{i}$ admits $\mathbb{C}$, and $C(\mathfrak{B}) \cap \mathfrak{F}=1$. Let $\mathfrak{S}_{i}=\mathfrak{F} \cap C\left(\mathfrak{B}_{i}\right)$, so that $\left|\mathfrak{E}: \mathfrak{E}_{i}\right|=2, i=1,2,3$.

Let $\mathfrak{X}$ be a subgroup of $\mathfrak{B}$ of index 2 such that $[\mathfrak{X}, \mathfrak{E}]=\mathfrak{Y}$ is of order 2. Let $\mathfrak{F}_{0}$ be any subgroup of $\mathfrak{E}$ of order 2 . Then $\mathfrak{B}=\mathfrak{B}_{1} \times \cdots \times \mathfrak{B}_{s}$, where each $\mathfrak{B}_{i}$ is an indecomposable $\mathfrak{E}_{0}$-group. Thus, $\left|\mathfrak{B}_{i}\right| \leqq 4,1 \leqq$ $i \leqq s$. Suppose $\left|\mathfrak{B}_{i}\right|=4, i=1,2,3$. Let $\tilde{\mathfrak{B}}=\mathfrak{B}_{1} \times \mathfrak{B}_{2} \times \mathfrak{B}_{3}$ and let $\mathfrak{B}_{0}=\mathfrak{X} \cap \tilde{\mathfrak{B}}$. Thus, $\left|\mathfrak{B}_{0}\right| \geqq 2^{5}$. Since $\left|\tilde{\mathfrak{B}} \cap C\left(\mathfrak{E}_{0}\right)\right|=2^{3}$, it follows that $\left|\left[\mathfrak{B}_{0}, \mathfrak{C}_{0}\right]\right| \geqq 2^{2}$, against $\left[\mathfrak{B}_{0}, \mathfrak{E}_{0}\right] \subseteq \mathfrak{Y}$. We conclude that there are at most 2 values of $i$ such that $\left|\mathfrak{B}_{i}\right|=4,1 \leqq i \leqq s$. 
On the other hand, $\sqrt{5}$ contains an element $C$ which inverts $\mathfrak{B}$. Since $|\mathfrak{B}|=p_{1} p_{2} p_{3}$ and since $\mathfrak{B}$ is faithfully represented on $\mathfrak{B}$, it follows that $|[\mathfrak{B}, \mathfrak{B}]| \geqq 2^{6}$. Thus, the preceding argument with $\mathfrak{C}_{0}=\langle C\rangle$ yields the desired contradiction.

Lemma 13.13. One of the following holds:

(a) $|\mathfrak{B}| \leqq 4$.

(b) If $G \in \mathfrak{B S}$ and $\left|\mathfrak{B}^{G}: \mathfrak{B}^{G} \cap \mathfrak{M}\right| \leqq 2$, then $\mathfrak{B}^{G} \subseteq \mathfrak{M}$.

(c) $|\mathfrak{B}|=2^{4}$ and $\boldsymbol{A}_{\circledast}(\mathfrak{B})$ contains a subgroup of index at most 2 which is isomorphic to $\Sigma_{3} \times \Sigma_{3}$.

Proof. Suppose neither (a) nor (b) holds. Choose $G$ in (S) such that $\left|\mathfrak{B}^{G}: \mathfrak{B}^{G} \cap \mathfrak{M}\right| \leqq 2$ and $\mathfrak{B}^{G} \nsubseteq \mathfrak{M}$. Set $\mathfrak{X}=\mathfrak{B}^{G} \cap \mathfrak{M}$, so that $\mathfrak{X}$ is of index 2 in $\mathfrak{B}^{G}$. Let $\mathfrak{X}_{0}=\mathfrak{X} \cap C(\mathfrak{B})$, and let $\mathfrak{Y}$ be a complement to $\mathfrak{X}_{0}$ in $\mathfrak{X}$. Suppose $|\mathfrak{Y}| \leqq 2$. Then $\mathfrak{X}_{0}$ is of index at most 4 in $\mathfrak{B}^{G}$. Since $|\mathfrak{B}| \geqq 8$, it follows from Lemma 13.11 that $C\left(\mathfrak{X}_{0}\right) \subseteq \mathfrak{M}^{a}$. In particular, $\mathfrak{B} \subseteq \mathfrak{M}^{a}$. By Lemma 13.7 applied to $\mathfrak{M}^{G}$, we get $\left[\mathfrak{B}, \mathfrak{B}^{G}\right]=1$. This yields $\mathfrak{B}^{G} \subseteq C(\mathfrak{B}) \subseteq N(\mathfrak{B})=\mathfrak{M}$, against our choice of $G$. We conclude that $|\mathfrak{Y}|=2^{y} \geqq 4$.

Since $\mathfrak{B}$ is 2-reducible in $\mathfrak{M}, \mathfrak{Y}$ acts faithfully on $\boldsymbol{O}_{2},(\mathfrak{M} / C(\mathfrak{B}))$. By Lemma 5.34, we can find abelian subgroup $\mathfrak{X}$ of $\boldsymbol{F}(\mathfrak{M} / \boldsymbol{C}(\mathfrak{B})$ ) such that $\mathfrak{U}=\mathfrak{U}_{1} \times \cdots \times \mathfrak{N}_{y}, \mathfrak{U}_{i}$ is of prime order $p_{i}, \mathfrak{N}_{i}$ admits $\mathfrak{Y}, 1 \leqq i \leqq y$, and such that $\mathfrak{Y}$ acts faithfully on $\mathfrak{X}$. Let $\mathfrak{Y}_{i}=C_{\mathfrak{y}}\left(\mathfrak{U}_{i}\right)$, so that $\left|\mathfrak{Y}: \mathfrak{Y}_{i}\right|=2$. Let $\mathfrak{B}_{i}=\left[\mathfrak{B}, \mathfrak{N}_{i}\right], \mathfrak{B}_{i}^{*}=\mathfrak{B}_{i} \cap \boldsymbol{C}\left(\mathfrak{Y}_{i}\right)$.

Suppose $\mathfrak{B}_{i}^{*}$ contains a four-subgroup 3 such that $3 \cap C(\mathfrak{Y})=1$. We will derive a contradiction from this assumption.

Since $\mathfrak{Y}_{i} \mathfrak{X}_{0}$ centralizes $\mathfrak{S}_{i}^{*}$ and since $\mathfrak{Y}_{i} \mathfrak{X}_{0}$ is of index 4 in $\mathfrak{B}^{\sigma}$, it follows that $\mathfrak{B}_{i}^{*} \subseteq \mathfrak{M}^{G}$, by Lemma 13.10 applied to $\mathfrak{M}^{G}$. In particular,

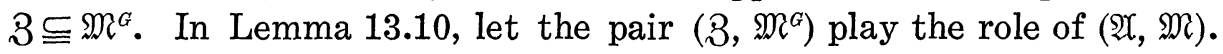
Now $3 \cap \boldsymbol{C}\left(\mathfrak{B}^{G}\right)=1$, since $3 \cap \boldsymbol{C}(\mathfrak{V})=1$ and $\mathfrak{Y} \subseteq \mathfrak{B}^{G}$. Also, 3 centralizes $\mathfrak{Y}_{i} \mathfrak{X}_{0}$, a subgroup of $\mathfrak{S}^{G}$ of index 4 . Choose $Y$ in $\mathfrak{X}-\mathfrak{Y}_{i} \mathfrak{X}_{0}$. Then by Lemma 13.10 , we can find $Z$ in 3 such that $C([Y, Z]) \subseteq \mathfrak{M}^{G}$. But $[Y, Z] \in \mathfrak{B}$, so we get $\mathfrak{B} \subseteq \mathfrak{M}^{G}$. Thus, Lemma 13.7 implies that $\left[\mathfrak{B}, \mathfrak{B}^{G}\right]=1$, against our choice of $G$. We conclude that 3 is not available.

Let $Y_{i}$ be an element of $\mathfrak{Y}-\mathfrak{Y}_{i}$. Since $\mathfrak{B}_{i}^{*} \cap \boldsymbol{C}(\mathfrak{Y})=\mathfrak{B}_{i}^{*} \cap \boldsymbol{C}\left(Y_{i}\right)$, it follows that $\mathfrak{B}_{i}^{*} \cap \boldsymbol{C}\left(Y_{i}\right)$ is of index 2 in $\mathfrak{B}_{i}^{*}$. Since $Y_{i}$ inverts $\mathfrak{N}_{i}$, we can find an element $A_{i}$ of $\mathfrak{M}$ such that $\mathfrak{Q}_{i}=\left\langle A_{i} C(\mathfrak{B})\right\rangle$, and such that $Y_{i}$ inverts $A_{i}$. Since $A_{i}$ has no fixed points on $\mathfrak{B}_{i}^{* \sharp}$, it follows that $\left|\mathfrak{B}_{i}^{*}\right|=4$.

We next argue that $\mathfrak{B}_{i}^{*} \subset \mathfrak{V}_{i}$. By construction, $\mathfrak{B}_{i}^{*} \leqq \mathfrak{V}_{i}$, so suppose by way of contradiction that $\mathfrak{B}_{i}^{*}=\mathfrak{B}_{i}$. Then Lemma 5.20 implies that $C(X) \subseteq \mathfrak{M}$ for all $X \in \mathfrak{B}_{i}^{*}$. In particular, $C\left(\left[\mathfrak{B}_{i}^{*}, Y_{i}\right]\right) \subseteq \mathfrak{M}$. But $\mathfrak{B}_{i}^{*}$ centralizes $\mathfrak{X}_{0} \mathfrak{Y}_{i}$, so $\mathfrak{B}_{i}^{*} \subseteq \mathfrak{M}^{G}$. This yields $\left[\mathfrak{B}_{i}^{*}, Y_{i}\right] \subseteq \mathfrak{B}^{G}$, so we get $\mathfrak{B}^{G} \subseteq \mathfrak{M}$, against our choice of $G$. 
We next show that $\left|\mathfrak{B}_{i}\right|=2^{4}$. Since $\mathfrak{B}_{i}^{*} \subset \mathfrak{B}_{i}$, it follows that $\left|\mathfrak{B}_{i}\right| \geqq 2^{4}$. Suppose by way of contradiction that $\left|\mathfrak{B}_{i}\right|>2^{4}$.

Set $\mathfrak{E}_{i}=\mathfrak{B}_{i}^{*} \cap C(\mathfrak{Y})=\mathfrak{B}_{i} \cap C(\mathfrak{Y})=\mathfrak{B}_{i} \cap C(\mathfrak{X})$, so that $\left|\mathfrak{F}_{i}\right|=2$. Also, set $\mathfrak{D}_{i}=\mathfrak{B}_{i} \cap N\left(\mathfrak{B}^{G}\right)$, so that $\mathfrak{D}_{i} \supseteqq \mathfrak{B}_{i}^{*}$. Since $\left[\mathfrak{D}_{i}, \mathfrak{B}^{G}\right] \subseteq \mathfrak{B}^{G}$, it follows that $\left[\mathfrak{D}_{i}, \mathfrak{X}\right] \subseteq \mathfrak{B}_{i} \cap \mathfrak{B}^{G} \subseteq \mathfrak{B}_{i} \cap \boldsymbol{C}(\mathfrak{X}) \subseteq \mathfrak{F}_{i}$, and so $\left[\mathfrak{D}_{i}, \mathfrak{X}\right]=\mathfrak{E}_{i}$. By Lemma 13.12 applied to $\mathfrak{M}^{G}$, it follows that $\left|\mathfrak{D}_{i}: \mathbb{E}_{i}\right| \leqq 4$.

By Lemma $13.6(\mathrm{c}), \mathfrak{B}^{G}$ contains a four-group 3 such that $C(Z) \subseteq \mathfrak{M}^{G}$ for all $Z$ in $3^{\sharp}$. Hence, $\mathfrak{X}$ contains an element $X$ with $C(X) \subseteq \mathfrak{M}^{a}$. Hence, $C(X) \cap \mathfrak{B}_{i} \leqq \mathfrak{D}_{i}$, so that $\left(C(X) \cap \mathfrak{B}_{i}\right) \mathfrak{B}_{i}^{*}$ has order at most 8 . First, suppose $X \in \mathfrak{X}_{0} \mathfrak{V}_{i}$. Since $\left|\mathfrak{B}_{i}\right|>2^{4}$, it follows that $\left|C(X) \cap \mathfrak{B}_{i}\right| \geqq 8$. Since $C(X) \cap \mathfrak{B}_{i}$ admits $\mathfrak{N}_{i}$, we get $\left|C(X) \cap \mathfrak{B}_{i}\right| \geqq 2^{4}$. We have just seen that this is not the case. Suppose $X \notin \mathfrak{X}_{0} \mathfrak{Y}_{i}$. Since $X$ does not centralize $\mathfrak{B}_{i}^{*}$, and since $\mathfrak{B}_{i}^{*} \cong \mathfrak{D}_{i}$, it follows that $\left|C(X) \cap \mathfrak{B}_{i}\right| \leqq 4$. This is impossible, since $\left|\mathfrak{B}_{i}\right|>2^{4}$. Both possibilities yield contradiction, so we conclude that $\left|\mathfrak{B}_{i}\right|=2^{4}, 1 \leqq i \leqq y$. Notice that among other things, we get that $\mathfrak{A}$ is an elementary of order $3^{y}$, since $\mathfrak{U}_{i}$ is faithfully represented on the four-group $\mathfrak{B}_{i}^{*}$.

Suppose by way of contradiction that $y \neq 2$. Since we have already shown that $y \geqq 2$, we get that $y \geqq 3$.

Let $\Re=O_{3^{\prime}}(\mathfrak{M})$. Since $\mathfrak{Y}$ is is faithfully represented on $\mathfrak{A}$, a 3-group, it follows that $\mathfrak{Y} \cap \Re=1$. Thus, $\mathfrak{Y}$ is faithfully represented on $\boldsymbol{O}_{3^{\prime}, 3}(\mathfrak{M}) / \Re$. By Lemma $5.34, \boldsymbol{O}_{\mathfrak{s}^{\prime}, 3}(\mathfrak{M})$ contains an elementary subgroup of order $3^{3}$, and so $3 \in \sigma$.

Now $\mathfrak{A}=\mathfrak{L} / \boldsymbol{C}(\mathfrak{B})$ for some subgroup $\mathbb{L}$ of $\mathfrak{M}$. Since $\mathbb{E}_{i}=\mathfrak{B}_{i} \cap \mathfrak{B}^{G}$ is of order 2 and since $C\left(\mathfrak{S}_{i}\right) \nsubseteq \mathfrak{M}$ it follows that $\boldsymbol{C}(\mathfrak{B})$ has cyclic $S_{3}$ subgroups. Thus, a $S_{3}$-subgroup $\mathfrak{R}_{3}$ of $\mathbb{R}$ has a cyclic normal subgroup $\mathfrak{Z}_{3} \cap C(\mathfrak{B})$ such that the factor group $\mathfrak{B}_{3} / \mathbb{R}_{3} \cap C(\mathfrak{B}) \cong \mathfrak{A}$ is elementary of order $3^{y}$. It follows that each non cyclic subgroup of $\mathfrak{\Omega}_{3}$ of order 9 is contained in $\mathscr{A}(3)$.

Since $C\left(\mathbb{E}_{i}\right) \nsubseteq \mathfrak{M}$, it follows that $\left|\boldsymbol{C}_{\mathfrak{r}}\left(\mathbb{E}_{i}\right)\right| \leqq 3,1 \leqq i \leqq y$. Since $\left|\mathfrak{B}_{i}\right|=2^{4}$, we have also $\left|\mathfrak{N}: C_{\mathfrak{r}}\left(\mathbb{E}_{i}\right)\right| \leqq 3^{2}$. We conclude that $\left|\boldsymbol{C}_{\mathfrak{r}}\left(\mathfrak{B}_{i}\right)\right|=3$, $\left|\mathfrak{A}: C_{\mathfrak{r}}\left(\mathfrak{B}_{i}\right)\right|=3^{2}, y=3$.

Since $\mathscr{Y}$ acts faithfully on $\mathfrak{A}$, the only subgroups of $\mathfrak{A}$ of order 3 which admit $\mathfrak{V}$ are $\mathfrak{N}_{1}, \mathfrak{N}_{2}, \mathfrak{N}_{3}$. Thus, we may assume that notation is chosen so that $\mathfrak{H}_{3}=C_{\mathfrak{r}}\left(\mathfrak{B}_{1}\right)$. Thus, $\mathfrak{A}_{2}$ does not centralize $\mathfrak{B}_{1}$. Suppose $\mathfrak{B}_{1} \cap \boldsymbol{C}\left(\mathfrak{H}_{2}\right) \neq 1$. Then $\mathfrak{B}_{1} \cap \boldsymbol{C}\left(\mathfrak{N}_{2}\right)$ and $\left[\mathfrak{B}_{1}, \mathfrak{N}_{2}\right]$ are both non trivial and both admit $\mathfrak{Y}$. Thus, $\mathfrak{B}_{1} \cap C(\mathfrak{Y})$ is non cyclic, since $\mathfrak{B}_{1}$ is the direct product of $\mathfrak{B}_{1} \cap C\left(\mathfrak{A}_{2}\right)$ and $\left[\mathfrak{V}_{1}, \mathfrak{N}_{2}\right]$. This is impossible, since $\left|\mathfrak{F}_{i}\right|=2,1 \leqq i \leqq y$. Hence, $\mathfrak{B}_{1} \cap \boldsymbol{C}\left(\mathfrak{A}_{2}\right)=1$. Since $\left|\mathfrak{B}_{2}\right|=2^{4}$, we conclude that $\mathfrak{B}_{1}=\mathfrak{B}_{2}$. By symmetry, we have $\mathfrak{B}_{1}=\mathfrak{B}_{2}=\mathfrak{B}_{3}$. This is absurd, since $\mathfrak{N}_{3}$ centralizes $\mathfrak{B}_{1}$. We conclude that $y=2$.

We next show that $3 \in \sigma$. Suppose false. Let $\mathfrak{S}_{\mathrm{C}}$ be a $S_{2^{\prime}}$-subgroup of $\mathfrak{M}$. Since $\mathfrak{S}$ has no elementary subgroup of order $3^{3}$, it follows from Lemma 0.8 .5 that $\mathfrak{S}_{\mathcal{C}}$ has a normal 3-complement. Let $\mathfrak{S}_{3}$ be a 
$S_{3}$-subgroup of $\mathfrak{S}_{2}$. Since $\mathfrak{A} \triangleleft \triangleleft \mathfrak{M} / C(\mathfrak{B})$, we have $\mathfrak{X} \leqq \mathfrak{S}_{3} C(\mathfrak{B}) / C(\mathfrak{B})$. Let $p \in \sigma$, and let $\mathfrak{S}_{p}$ be a $S_{p}$-subgroup of $\mathfrak{S}_{\mathfrak{C}}$ permutable with $\mathfrak{S}_{3}$. Thus, $\mathfrak{S}_{p} \triangleleft \mathfrak{S}_{3} \mathfrak{H}_{p}$.

By construction, $\mathfrak{U} \subseteq \boldsymbol{F}(\mathfrak{M} / \boldsymbol{C}(\mathfrak{B}))$. Thus, $\mathfrak{S C}_{p} \boldsymbol{C}(\mathfrak{B}) / \boldsymbol{C}(\mathfrak{B})$ centralizes 2. In particular, $\mathfrak{S}_{p}$ normalizes $\mathfrak{B}_{1}$. Hence, $\mathfrak{S}_{p} \cap \boldsymbol{C}\left(\mathfrak{B}_{1}\right)$ contains an element of $\mathscr{\Omega}(p)$. This is impossible, since $C\left(\mathbb{S}_{1}\right) \nsubseteq \mathfrak{M}$. Hence, $3 \in \sigma$.

Let $3=\mathfrak{B} \cap C(\mathfrak{Z})$. Since $3 \in \sigma$, it follows that $C(Z) \subseteq \mathfrak{M}$ for all $Z$ in $3^{*}$. Since $C\left(\mathfrak{X}_{0}\right) \supseteqq \mathfrak{B}$, it follows that $\boldsymbol{C}\left(\mathfrak{X}_{0}\right) \nsubseteq \mathfrak{M}^{G}$. Hence, $\mathfrak{X}_{0} \cap \mathfrak{Z}^{G}=1$. This implies that $|3| \leqq 8$, since $\left|\mathfrak{B}^{\alpha}: \mathfrak{X}_{0}\right|=8$.

We next show that $\mathfrak{B}_{1}=\mathfrak{B}_{2}$. Suppose false. Since $\left|\mathfrak{B}_{1}\right|=\left|\mathfrak{B}_{2}\right|=2^{4}$, and since $\mathfrak{B}_{1}$ admits $\mathfrak{N}_{2}$, it follows that $\mathfrak{B}_{1} \cap \boldsymbol{C}\left(\mathfrak{N}_{2}\right) \neq 1$. Since $\mathfrak{S}_{1} \cap \boldsymbol{C}(\mathfrak{Y})=$ $\mathfrak{S}_{1}$ is of order 2 , it follows that $\mathfrak{A}_{2}$ centralizes $\mathfrak{B}_{1}$. Hence, $\mathfrak{B}_{1} \cap \mathfrak{B}_{2}=1$.

Since $\mathfrak{B}_{1} \cap \mathfrak{B}_{2}=1$, it follows that $\mathfrak{B}_{1} \mathfrak{B}_{2}=\mathfrak{B}_{1} \times \mathfrak{B}_{2}=[\mathfrak{B}, \mathfrak{X}]$. Thus, $\mathfrak{N}=\mathfrak{B}_{1} \times \mathfrak{B}_{2} \times \mathfrak{3}$. Let $\mathfrak{D}=\boldsymbol{O}_{3^{\prime}}(\boldsymbol{F}(\mathfrak{M} / \boldsymbol{C}(\mathfrak{B})))$. We will show that $\mathfrak{D}$ centralizes $\mathfrak{B}_{1}, i=1,2$. Since $\left|\mathfrak{B}_{1}\right|=2^{4}$, and since $\mathfrak{D}$ centralizes $\mathfrak{A}$, we may assume by way of contradiction that $\left|\mathfrak{D}: \mathfrak{D} \cap C\left(\mathfrak{B}_{1}\right)\right|=5$. Since $\mathfrak{D} / \mathfrak{D} \cap C\left(\mathfrak{B}_{1}\right)$ admits $\mathfrak{Y}$, there is an element $Y$ in $\mathfrak{V}$ which centralizes $\mathfrak{D} / \mathfrak{D} \cap \boldsymbol{C}\left(\mathfrak{B}_{1}\right)$. Thus, $\mathfrak{D}$ normalizes $\mathfrak{B}_{1} \cap \boldsymbol{C}(Y)$. Since $\mathfrak{V}$ acts faithfully on $\mathfrak{B}_{1}$, it follows that $\left|\mathfrak{B}_{1} \cap C(Y)\right|<2^{4}$, so that $\mathfrak{D}$ centralizes $\mathfrak{B}_{1} \cap \boldsymbol{C}(Y)$. Hence, $\mathfrak{D}$ centralizes $\mathfrak{B}_{i}, i=1$, 2. Since $\boldsymbol{F}(\mathfrak{M} / C(\mathfrak{B}))$ is faithfully represented on $\mathfrak{B}$, it follows that $\boldsymbol{O}_{3^{\prime}}(\boldsymbol{F}(\mathfrak{M} / C(\mathfrak{B})))$ is faithfully represented on 3 . Hence, $O_{3^{\prime}}(F(\mathfrak{M} / C(\mathfrak{B})))=1$ or 7 , since $|3| \leqq 8$. In particular, $\boldsymbol{O}_{3^{\prime}}(\boldsymbol{F}(\mathfrak{M} / C(\mathfrak{B})))$ is cyclic. Since $3 \in \sigma, 3 \nmid\left|\mathfrak{M}: \mathfrak{M}^{\prime}\right|$. This implies that a $S_{3^{-}}$-subgroup of $\mathfrak{M} / C(\mathfrak{B})$ centralizes $\boldsymbol{O}_{3^{\prime}}(\boldsymbol{F}(\mathfrak{M} / C(\mathfrak{B})))$.

Let $\mathfrak{K}=O_{3}(\mathfrak{M} / C(\mathfrak{N})) \supseteqq \mathfrak{A}$, and let $\mathfrak{S}_{0}=N_{\mathfrak{F}}(\mathfrak{U})$. Thus, $\mathfrak{S}_{0}$ admits $\mathfrak{Y}$. Hence, $\mathfrak{U} \cap \boldsymbol{C}\left(\mathfrak{S}_{0}\right)$ admits $\mathfrak{Y}$. Suppose $\mathfrak{X} \cap \boldsymbol{C}\left(\mathfrak{S}_{0}\right) \subset \mathfrak{X}$. Since $\mathfrak{X}_{1}, \mathfrak{A}_{2}$ are the only subgroups of $\mathfrak{X}$ of order 3 which admit $\mathfrak{Y}$, we may assume notation is chosen so that $\mathfrak{U} \cap \boldsymbol{C}\left(\mathfrak{S}_{0}\right)=\mathfrak{N}_{1}$. Hence, $\mathfrak{S}_{0}$ normalizes $\mathfrak{B}_{1}$. Since $\mathfrak{N}_{2}$ centralizes $\mathfrak{B}_{1}$, and since $C\left(\mathfrak{B}_{1}\right) \cap \mathfrak{S}_{0} \triangleleft \mathfrak{S}_{0}$, it follows that

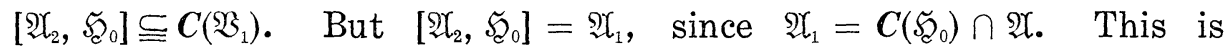
absurd, since $\mathfrak{X}_{1}$ does not centralize $\mathfrak{B}_{1}$. Hence, $\mathfrak{F}_{0}$ centralizes $\mathfrak{X}$. Suppose $\mathfrak{S}_{0}$ contains an elementary subgroup $\mathfrak{U}^{*}$ of order $3^{3}$. We may assume that $\mathfrak{Y} \subset \mathfrak{U}^{*}$ and that $\mathfrak{U}^{*}$ admits $\mathfrak{Y}$. Let $\mathfrak{U}_{3}$ be a subgroup of $\mathfrak{Y}^{*}$ of order 3 which admits $\mathfrak{Y}$ and is a complement to $\mathfrak{X}$ in $\mathfrak{Y} *$. If $\mathfrak{A}_{3}$ is not centralized by $\mathfrak{Y}$, then by replacing $\mathfrak{A}$ by another subgroup of order 9 which contains $\mathfrak{A}_{3}$ and on which $\mathfrak{Y}$ acts faithfully, we see that we get $\left[\mathfrak{B}, \mathfrak{U}_{3}\right]=2^{4}$. Thus, $\mathfrak{U}_{3}$ centralizes either $\mathfrak{B}_{1}$ or $\mathfrak{B}_{2}$. But in this case, $\mathfrak{U}^{*} \cap \boldsymbol{C}\left(\mathfrak{B}_{1}\right)$ or $\mathfrak{U}^{*} \cap \boldsymbol{C}\left(\mathfrak{B}_{2}\right)$ is of order 9 . This is impossible, since $3 \in \sigma$. Hence, $\mathfrak{Y}$ centralizes $\mathfrak{U}_{3}$. Since $\left|\mathfrak{S}_{i}\right|=2, i=1,2$, it follows that $\mathfrak{N}_{3}$ centralizes $\widetilde{F}_{1}$ and $\mathfrak{F}_{2}$. Thus, $\boldsymbol{C}_{\mathfrak{M}}\left(\mathfrak{S}_{1}\right)$ or $\boldsymbol{C}_{\mathfrak{M}}\left(\mathfrak{S}_{2}\right)$ contains an element of $\mathscr{( 3 )}$. This is impossible, since $C\left(\mathfrak{C}_{i}\right) \nsubseteq \mathfrak{M}$. It follows that $\mathfrak{S}_{0}$ contains no elementary subgroup of order $3^{3}$. Since $\mathfrak{V} \subseteq \mathbb{Z}\left(\mathfrak{F}_{0}\right)$, it follows that $\mathfrak{U}=\Omega_{1}\left(\mathfrak{S}_{0}\right)$ char $\mathfrak{S}_{0}$. Hence, $\mathfrak{S}_{0}=\mathfrak{S}_{2}$, which implies that $\mathfrak{N} \triangleleft \mathfrak{M} / C(\mathfrak{B})$. Also, since $\left|\mathfrak{B}_{i}\right|=2^{4}, i=1,2$, it follows that $\mathfrak{N}_{i}$ is not 
contained in any cyclic subgroup of $\mathfrak{S}$ of order 9 . This in turn implies that $\mathfrak{A}=\mathfrak{S}$.

Let $\mathfrak{B}^{*}$ be a $S_{3}$-subgroup of $\mathfrak{M} / C(\mathfrak{B})$. As we have already shown, $\mathfrak{B}^{*}$ centralizes $\boldsymbol{O}_{3^{\prime}}(\boldsymbol{F}(\mathfrak{M} / \boldsymbol{C}(\mathfrak{B})))$. Now $\mathfrak{U}_{1}, \mathfrak{A}_{2}$ are the only subgroups $\mathfrak{U}$ of $\mathfrak{A}$ of order 3 which satisfy $|[\mathfrak{B}, \mathfrak{M}]|=2^{4}$. Hence, $\mathfrak{B}^{*}$ centralizes $\mathfrak{2}$. Thus, $\mathfrak{B}^{*}$ centralizes $\boldsymbol{F}(\mathfrak{M} / C(\mathfrak{B}))$, so $\mathfrak{B}^{*}=\mathfrak{2}$.

Since $\mathfrak{A}$ is a $S_{3}$-subgroup of $\mathfrak{M} / C(\mathfrak{B})$, and since $3 \in \sigma$, it follows that $C(\mathfrak{B})$ contains a non trivial cyclic $S_{3}$-subgroup. This implies that the inverse image of $\mathfrak{U}_{1}$ in $\mathfrak{M}$ contains a non cyclic $S_{3}$-subgroup, so contains an element of $\mathscr{A}(3)$. This violates $C\left(\mathbb{S}_{1}\right) \nsubseteq \mathfrak{M}$. We conclude that $\mathfrak{B}_{1}=\mathfrak{B}_{2}$.

Suppose $\mathfrak{U} \triangleleft \mathfrak{M}, \mathfrak{u} \subseteq \mathfrak{B}$, and $|\mathfrak{M}| \leqq 8$. We will show that $\mathfrak{U}=1$. Suppose false. Since $|\mathfrak{u}| \leqq 8, C_{\mathfrak{m}}(\mathfrak{U})$ contains an element of $\mathscr{A}(3)$, so $\mathfrak{u} \# \cong \mathscr{J}$.

\section{Case 1. $|\mathfrak{u}|=2$.}

Since $C(\mathfrak{X}) \subseteq \mathfrak{M}^{\alpha}$, we get $\mathfrak{u} \subseteq \mathfrak{M}^{\alpha}$. Since $C(\mathfrak{U}) \subseteq \mathfrak{M}$, we get $\mathfrak{u}^{\alpha} \subseteq \mathfrak{M}$. Hence, $\mathfrak{l}^{G} \leqq \mathfrak{X}$. Since $\boldsymbol{C}\left(\mathfrak{u}^{G}\right)=\mathfrak{M}^{G}$, we get $\mathfrak{u}^{G} \nsubseteq \mathfrak{X}_{0}$. Thus, we may assume that $\mathfrak{u}^{G} \subseteq \mathfrak{Y}$. Thus, either $\mathfrak{U}^{G} \neq \mathfrak{Y}_{1}$ or $\mathfrak{u}^{G} \neq \mathfrak{V}_{2}$. Suppose notation has been chosen so that $\mathfrak{U}^{G} \neq \mathfrak{V}_{1}$. Then $\mathfrak{U}^{G}$ does not centralize $\mathfrak{B}_{1}^{*}=\mathfrak{B}_{1} \cap C\left(\mathfrak{Y}_{1}\right)$. But $\mathfrak{B}_{1}^{*} \subseteq \mathfrak{M}^{G}$, since $C\left(\mathfrak{X}_{0} \mathfrak{Y}_{1}\right) \subseteq \mathfrak{M}^{G}$. This contradiction shows that this case does not occur.

\section{Case 2. $|\mathfrak{u}| \geqq 4$.}

Since $\mathfrak{X}$ normalizes $\mathfrak{U}, \mathfrak{X}$ contains a subgroup $\tilde{X}$ of index 2 such that $\mathfrak{U} \cap C(\tilde{\mathfrak{X}})$ is non cyclic. Since $\boldsymbol{C}(\tilde{\mathfrak{X}}) \subseteq \mathfrak{M}^{G}$, we get $\mathfrak{U} \cap \boldsymbol{C}(\tilde{\mathfrak{X}}) \subseteq \mathfrak{M}^{G}$. Since $\mathfrak{U} \cap \boldsymbol{C}(\tilde{\mathfrak{X}})$ is non cyclic, we can choose an involution $U$ in $\mathfrak{u} \cap \boldsymbol{C}(\tilde{\mathfrak{X}})$ such that $\boldsymbol{C}(U) \cap \mathfrak{u}^{\sigma}$ is non cyclic. Hence, $\mathfrak{X} \cap \mathfrak{u}^{\sigma}$ is non cyclic. Since $\mathfrak{X}_{0} \cap \mathfrak{U}^{G}=1$, we may assume that $\mathfrak{Y}=\mathfrak{X} \cap \mathfrak{U}^{G}$. Then since $\mathfrak{B}_{1}^{*} \subseteq \mathfrak{M}^{G}$, we get $\mathfrak{\Im}_{1}=\left[\mathfrak{Y}, \mathfrak{B}_{1}^{*}\right] \leqq \mathfrak{U}^{G}$. This gives $C\left(\mathfrak{\mho}_{1}\right) \subseteq \mathfrak{M}^{G}$, which forces $\mathfrak{B} \cong \mathfrak{M}^{G}$. This contradiction shows that this case does not occur, and completes a proof that $\mathfrak{U}=1$.

Suppose $\mathfrak{B}_{0}$ is a subgroup of $\boldsymbol{F}(\mathfrak{M} / \boldsymbol{C}(\mathfrak{B}))$ of order 3 which admits $\mathfrak{Y}$ and is not centralized by $\mathfrak{Y}$. Let $\mathfrak{Y}_{0}=C_{\mathfrak{y}}\left(\mathfrak{B}_{0}\right)$ so that $\left|\mathfrak{Y}_{0}\right|=2$. Since $\mathfrak{Y}_{0}$ is faithfully represented on $\boldsymbol{O}_{3}(\mathfrak{M} / C(\mathfrak{B}))$, $\mathfrak{Y}_{0}$ is faithfully represented on $\boldsymbol{C}\left(\mathfrak{B}_{0}\right) \cap \boldsymbol{O}_{3}(\mathfrak{M} / \boldsymbol{C}(\mathfrak{B}))$. Thus, $\mathfrak{B}_{0}$ is contained in a subgroup $\tilde{\mathfrak{B}}$ of $\boldsymbol{F}(\mathfrak{M} / \boldsymbol{C}(\mathfrak{B}))$ of order 9 on which $\mathfrak{Y}$ acts faithfully. Replacing $\mathfrak{Q}$ by $\tilde{\mathfrak{B}}$, we see that $\left|\left[\mathfrak{B}_{0}, \mathfrak{B}\right]\right|=2^{4}$.

Set $\mathfrak{F}=\boldsymbol{O}_{3}(\mathfrak{M} / C(\mathfrak{B}))$. Suppose $\mathfrak{B}_{0}$ is a subgroup of $\mathfrak{S}^{\prime} \cap \boldsymbol{Z}(\mathfrak{S})$ of order 3 which admits $\mathfrak{Y}$. If $\left[\mathfrak{B}_{0}, \mathfrak{Y}\right] \neq 1$, then $\mathfrak{B}=\left[\mathfrak{B}, \mathfrak{B}_{0}\right] \times \mathfrak{B} \cap \boldsymbol{C}\left(\mathfrak{B}_{0}\right)$ and $\mathfrak{S}$ normalizes both $\left[\mathfrak{B}, \mathfrak{B}_{0}\right]$ and $\mathfrak{B} \cap \boldsymbol{C}\left(\mathfrak{B}_{0}\right)$. Since $\left|\left[\mathfrak{B}, \mathfrak{B}_{0}\right]\right|=2^{4}$, $\mathfrak{S}^{\prime}$ centralizes $\left[\mathfrak{B}, \mathfrak{B}_{0}\right]$. This is absurd, since $\mathfrak{B}_{0}$ does not contralize $\left[\mathfrak{B}, \mathfrak{B}_{0}\right]$. 
Suppose $\left[\mathfrak{B}_{0}, \mathfrak{Y}\right]=1$. Since $\mathfrak{B}_{0} \subseteq Z(\mathfrak{E}), \mathfrak{B}_{0}$ centralizes $\mathfrak{B}$, so $\mathfrak{B}_{0}$ normalizes $\mathfrak{B}_{1}$. Hence, $\mathfrak{B}_{0}$ centralizes $\mathfrak{B}_{1} \cap \boldsymbol{C}(\mathfrak{Y})=\mathfrak{V}_{1}$, and so $\mathfrak{B}_{0}$ centralizes $\mathfrak{B}_{1}^{*}$ and $\mathfrak{B}_{2}^{*}$. Since $\left\langle\mathfrak{B}_{1}^{*}, \mathfrak{B}_{2}^{*}\right\rangle=8, \mathfrak{B}_{0}$ centralizes $\mathfrak{B}_{1}$. Since $\left|\left[\mathfrak{B}, \mathfrak{B}_{0}\right]\right| \leqq 8$, it follows that $\mathfrak{S}^{\prime}$ centralizes $\left[\mathfrak{B}, \mathfrak{B}_{0}\right]$. This is also a contradiction. Thus, $\mathfrak{B}_{0}$ does not exist. This implies that $\mathfrak{K}$ is abelian. Since $|\mathfrak{B}| \leqq 2^{7}$, it follows that either $\mathfrak{U}=\mathfrak{F}$ or $\mathfrak{S C}$ is elementary of order $3^{3}$.

Now $\mathfrak{U}=\mathfrak{Q} / \boldsymbol{C}(\mathfrak{B})$ for some subgroup $\mathbb{Q}$ of $\mathfrak{M}$. We argue that $\mathfrak{Z}$ contains an element of $\mathscr{A}(3)$. If $\mathfrak{E}=\mathfrak{A}$, then a $S_{3}$-subgroup of $\mathfrak{Q}$ is normalized by some $S_{3}$-subgroup of $\mathfrak{M}$, so we are done. Suppose $\mathfrak{A} \subset \mathfrak{F}$, so that $\mathfrak{S}$ is elementary of order $3^{3}$. If $3 \nmid|\boldsymbol{C}(\mathfrak{B})|$, we are done. We may assume that a $S_{3}$-subgroup of $C(\mathfrak{B})$ is a non identity cyclic group. Thus, a $S_{3}$-subgroup $\mathfrak{L}_{3}$ of $\mathbb{R}$ has a cyclic normal subgroup $\mathfrak{R}_{3} \cap \boldsymbol{C}(\mathfrak{B})$ with $\mathfrak{R}_{3} / \mathfrak{R}_{3} \cap \boldsymbol{C}(\mathfrak{B})$ elementary of order $3^{3}$. It follows that every non cyclic subgroup of $\mathfrak{R}_{3}$ of order 9 is in $\mathfrak{U}(3)$. To obtain this conclusion, we have used the fact that $\mathfrak{Y}$ acts faithfully on $\mathfrak{Y}$.

We next show that $3=1$. Suppose false. Let $|B|=2^{z}, 1 \leqq z \leqq 3$. Since $\mathfrak{M}$ contains no non identity normal subgroup $\mathfrak{U}$ of order $\leqq 8$, it follows that $z=2,|\mathfrak{B}|=2^{6}$.

Since $\mathfrak{X}$ centralizes 3 , we see that $\mathcal{C}(Z) \subseteq \mathfrak{M}$ for all $Z$ in $Z^{\sharp}$. Since $|3|=4$ and since $\mathfrak{Y}$ normalizes 3 , we can find a subgroup $\tilde{X}$ of index 2 in $\mathfrak{X}$ which centralizes 3 . Since $C(\tilde{\mathfrak{X}}) \subseteq \mathfrak{M}^{a}$, we get $3 \subseteq \mathfrak{M}^{a}$. Since $\left\langle\mathfrak{B}_{1}^{*}, \mathfrak{B}_{2}^{*}\right\rangle \subseteq \mathfrak{M}^{G}$, it follows that $\mathfrak{B} \cap \mathfrak{M}^{G}$ is of order $2^{5}$. By all the previous argument with $\left(\mathfrak{B} \cap \mathfrak{M}^{G}, \mathfrak{M}^{G}\right)$ in the role of $(\mathfrak{X}, \mathfrak{M})$, we conclude that $\left.\mathfrak{B} \cap \mathfrak{M}^{G} \cap \boldsymbol{C}^{\prime} \mathfrak{B}^{a}\right)$ is of index 4 in $\mathfrak{B} \cap \mathfrak{M}^{\text {s }}$. Now $\mathfrak{S}_{1}=\mathfrak{C}_{2}$ since $\mathfrak{B}_{1}=\mathfrak{B}_{2}$. Hence, $\left|\left\langle\mathfrak{B}_{1}^{*}, \mathfrak{B}_{2}^{*}\right\rangle: \mathfrak{V}_{1}\right|=4$. Hence,

$$
\mathfrak{B} \cap \mathfrak{M}^{a}=\left\langle\mathfrak{B} \cap \mathfrak{M}^{G} \cap \boldsymbol{C}\left(\mathfrak{B}^{G}\right), \mathfrak{B}_{1}^{*}, \mathfrak{B}_{2}^{*}\right\rangle \text {. }
$$

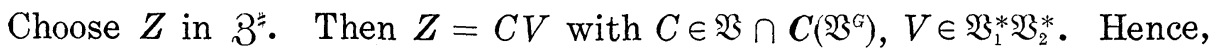
$[\mathfrak{X}, Z]=[\mathfrak{X}, V] \subseteq \mathfrak{B}_{1}$. Since $\mathfrak{X}$ normalizes 3 , we get $[Z, \mathfrak{X}] \subseteq \mathfrak{B}_{1} \cap 3=1$. Hence, 3 centralizes $\mathfrak{X}$. Since $3 \cap C\left(\mathfrak{B}^{a}\right)=1,3$ is faithfully represented on $\boldsymbol{O}_{2},\left(\mathfrak{M}^{G} / \boldsymbol{C}\left(\mathfrak{B}^{G}\right)\right)$. This is impossible, since 3 is a four-group which centralizes a hyperplane $\mathfrak{X}$ of $\mathfrak{B}^{G}$. We conclude that $3=1$.

Since $3=1$, we have $\mathfrak{B}=\mathfrak{B}_{1}$ of order $2^{4}$. Since Aut (פB) has no element of order 8 , it follows that a $S_{2}$-subgroup $\mathfrak{M}_{2}$ of $\mathfrak{M} / \boldsymbol{C}(\mathfrak{B})$ is of exponent 2 or 4 . Since $\mathfrak{M}_{2}$ is isomorphic to a subgroup of Aut (I), it follows that $\mathfrak{M}_{2}$ is either a four-group or a dihedral group of order 8. In any case, $\mathfrak{M} / \boldsymbol{C}(\mathfrak{B})$ has a subgroup of index at most 2 which is isomorphic to $\Sigma_{3} \times \Sigma_{3}$. The proof is complete.

Lemma 13.14. If $|\mathfrak{B}|=2^{4}$, then for each $V$ in $\mathfrak{B}^{\ddagger}, \mathfrak{B} \subseteq \boldsymbol{O}_{2}(C(V))$.

Proof. Suppose false, and $V$ in $\mathfrak{B}^{\sharp}$ is chosen so that $\mathfrak{B} \nsubseteq \boldsymbol{O}_{2}(\boldsymbol{C}(V))$. In particular, we have $C(V) \nsubseteq \mathfrak{M}$. Let $(\xi$ be an elementary subgroup of $\mathfrak{M}$ of order $p^{3}, p \in \sigma$. Let $\mathfrak{A}=\boldsymbol{C}(\mathfrak{B}) \cap \mathfrak{F}$. Since $\boldsymbol{C}(V) \nsubseteq \mathfrak{M}$, $\mathfrak{Q}$ is 
cyclic. Thus, $|\mathfrak{A}|=3$, since $|\mathfrak{B}|=2^{4}$. Since $C(\mathfrak{B})$ has cyclic $S_{3}$-subgroups, it follows that if $\mathfrak{P}$ is a $S_{3}$-subgroup of $\mathfrak{M}$ which contains $\mathbb{F}$, then $\mathbb{F}=\Omega_{1}(\mathfrak{P})$. Hence, every non cyclic 3 -subgroup of $\mathbb{B S}$ is in $\mathscr{A}^{*}(\mathbb{S})$. This implies that if $\Omega$ is a solvable subgroup of $\mathbb{B}$ which contains $\mathfrak{A}$ and also contains non cyclic $S_{3}$-subgroups, then $\Re \subseteq \mathfrak{M}$. Namely, since $C(\mathfrak{X}) \subseteq \mathfrak{M}$, we get that $\Re \cap \mathfrak{M}$ has non cyclic $S_{3}$-subgroups, whence $\mathfrak{R} \subseteq \mathfrak{M}$.

Let $\mathfrak{F}_{0}$ be a complement to $\mathfrak{A}$ in $\mathfrak{F}$. Thus, with a suitable choice of notation, $\mathfrak{F}_{0}=\mathfrak{F}_{1} \times \mathfrak{F}_{2}, \mathfrak{B}=\mathfrak{B}_{1} \times \mathfrak{B}_{2}$, and $\mathfrak{F}_{0} \mathfrak{B}=\mathfrak{F}_{1} \mathfrak{B}_{2} \times \mathfrak{F}_{2} \mathfrak{B}_{1} \cong A_{4} \times A_{4}$. There are exactly 3 orbits of $\mathfrak{B}^{\sharp}$ under the action of $\mathfrak{F}_{0}$, namely, $\mathfrak{B}_{1}^{\#}, \mathfrak{B}_{2}^{\sharp}$, and $\mathfrak{B}_{1}^{\sharp} \mathfrak{B}_{2}^{\sharp}$. Clearly, $V \in \mathfrak{B}_{1}^{\sharp} \mathfrak{B}_{2}^{\#}$. Furthermore, $\mathfrak{B}_{1}^{\sharp} \mathfrak{B}_{2}^{\sharp}$ is a conjugacy class of $\mathfrak{M}$, since $C(V) \nsubseteq \mathfrak{M}$. This implies that each element of $\mathfrak{B}_{1}^{\sharp} \mathfrak{B}_{2}^{\#}$ is centralized by a $S_{2}$-subgroup of $\mathfrak{M}$. Let $\mathfrak{K}$ be a $S_{2,3}$-subgroup of $C_{\mathfrak{m}}(V)$ which contains $\mathfrak{X}$, and let $\mathfrak{S}^{*}$ be a $S_{2,3}$-subgroup of $C(V)$ containing $\mathfrak{S}$. Since $C(\mathfrak{N}) \subseteq \mathfrak{M}$ and since $S_{3}$-subgroups of $C(V)$ are cyclic, it follows that $\mathfrak{S}=\mathfrak{S}^{*}$. Since $\mathfrak{B} \nsubseteq \boldsymbol{O}_{2}(\boldsymbol{C}(V))$, there are a prime $p \geqq 5$ and a $S_{p}$-subgroup $\mathfrak{F}_{p}$ of $\mathbb{E}=C(V)$ such that $\mathfrak{E}_{p}$ is permutable with $\mathfrak{S}$ and such that $\mathfrak{B} \nsubseteq \boldsymbol{O}_{2}\left(\mathfrak{S C C}_{\mathcal{C}}\right)$. Let $\mathfrak{S}_{2}, \mathfrak{S}_{3}$ be Sylow subgroups of $\mathfrak{S}_{2}$ which are permutable with $\mathfrak{E}_{p}$. We assume without loss of generality that $\mathfrak{A} \subseteq \mathfrak{S}_{3}$. Since $\mathfrak{S}_{3}$ is cyclic, we have $\mathfrak{E}_{p} \triangleleft \mathfrak{S}_{3} \mathfrak{E}_{p}$. Let $\mathfrak{D}$ be a subgroup of $\mathfrak{S}_{p}$ which is permutable with $\mathfrak{S}_{2}$ and with $\mathfrak{S}_{3}$ and is minimal subject to $\mathfrak{B} \nsubseteq \boldsymbol{O}_{2}(\mathfrak{S} \mathfrak{D})$. Let $\mathfrak{F}=\boldsymbol{O}_{2}(\mathfrak{C} \mathfrak{D})$. Since $\mathfrak{B} \nsubseteq \mathfrak{F}$, and since $\boldsymbol{O}_{2^{\prime}}(\mathfrak{S C D})=1$, it follows that $\mathfrak{B}$ acts non trivially on $\boldsymbol{O}_{2,2^{\prime}}(\mathfrak{S} \mathfrak{D}) / \mathfrak{F}$. Minimality of $\mathfrak{D}$ forces $\mathfrak{D} \subseteq \boldsymbol{O}_{2,2^{\prime}}(\mathfrak{S C D})$. Hence, $\mathfrak{F} \mathfrak{D} \triangleleft \mathfrak{S C D}$.

Choose $I \in \mathfrak{i}-\mathfrak{F}$. Since $|\mathfrak{B}|=2^{4}$, we have $|\mathfrak{B} \cap \mathfrak{F}| \leqq 2^{3}$. Since $p \geqq 5, V \in Z(\mathfrak{C})$, and $\mathfrak{B} \triangleleft \mathfrak{B} \mathfrak{F}$, it follows that $p=5$ and that $[\mathfrak{F} \mathfrak{D}, I] \cap \mathfrak{D}$ has order 5 , while $|\mathfrak{B} \cap \mathfrak{F}|=2^{3}$. Thus, $\langle I, \mathfrak{F}\rangle / \mathfrak{F} \in Z\left(\mathfrak{S}_{2} / \mathfrak{F}\right)$, and so

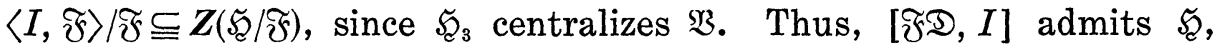
and so $|\mathfrak{D}|=5$. This implies that $\mathfrak{S}_{3}$ centralizes $\mathfrak{D}$, so $\mathfrak{D} \subseteq C(\mathfrak{R}) \subseteq \mathfrak{M}$. This is false, since by construction, $\mathfrak{D} \nsubseteq \mathfrak{M}$. The proof is complete.

\section{Lemma 13.15. Either (a) or (b) of Lemma 13.13 holds.}

Proof. Suppose false. Then by (c), $|\mathfrak{B}|=2^{4}$. Choose $G$ in (8) such that $\mathfrak{X}=\mathfrak{M} \cap \mathfrak{B}^{G}$ is of order 8. Let $\mathfrak{X}_{0}=\mathfrak{X} \cap C(\mathfrak{B})$. Thus, $\left|\mathfrak{X}_{0}\right|=2$. Also, $C(\mathfrak{X}) \cap \mathfrak{B}=\mathbb{E}$ is of order 2. Thus, $\sqrt{ }$ is in the center of a $S_{2^{-}}$ subgroup of $\mathfrak{M}$. Since $\mathfrak{B} \cap \mathfrak{M}^{G}$ is also of order 8 , it follows that $\mathfrak{F}$ is in the center of a $S_{2}$-subgroup of $\mathfrak{M}^{G}$. By Lemma 13.14, we have $\mathfrak{B} \subseteq \mathrm{O}_{2}(C(\mathbb{C}))$, $\mathfrak{B}^{G} \subseteq \mathrm{O}_{2}(C(\mathbb{C}))$. Since $C(\mathbb{C})$ contains a $S_{2}$-subgroup of $\mathfrak{M}$, we have $\boldsymbol{O}_{2}(\boldsymbol{C}(\mathfrak{C})) \subseteq \mathfrak{M}$. Hence, $\mathfrak{B}^{G} \subseteq \mathfrak{M}$, against $\left|\mathfrak{B}^{G}: \mathfrak{X}\right|=2$.

LEMma 13.16. One of the following holds:

(a) $|\mathfrak{B}| \leqq 4$.

(b) $\quad N\left(\mathfrak{B}^{*}\right) \subseteq \mathfrak{M}$. 
(c ) $\mathfrak{M}=\boldsymbol{N}_{\mathfrak{M}}\left(\mathfrak{B}^{*}\right) \cdot \boldsymbol{N}_{\mathfrak{n}}\left(\mathfrak{W}_{0}\right)$, where $\mathfrak{W}_{0}=\boldsymbol{Z}\left(\mathfrak{W}_{1}\right)$ and $\mathfrak{W}_{1}$ is the subgroup of $\mathfrak{I}$ generated by its subgroups $\mathfrak{U}$ with the property that for some $G$ in $\mathfrak{B S}, \mathfrak{l}$ is a subgroup of $\mathfrak{B}^{G}$ of index at most 2.

Proof. Suppose neither (a) nor (b) holds. Since (b) does not hold, it follows that (b)(i) of Lemma 13.8 holds. Suppose that (c) fails, too. By Lemma 0.7.7, it follows that for some odd prime $q$, M contains a $q$-group $\mathfrak{Q}$ with the properties that

(i) $\mathfrak{Q}$ is permutable with $\mathfrak{I}$,

(ii) $\mathfrak{I} \mathfrak{\Omega} \neq N_{\mathfrak{x} \mathbb{N}}\left(\mathfrak{B}^{*}\right) N_{\mathfrak{x}}\left(\mathfrak{W}_{0}\right)$,

(iii) the 2-length of $\mathfrak{T} \cong$ is at most 2 .

We assume that notation is chosen so that $\Omega$ is of minimal order subject to (i), (ii), (iii). Let $\mathfrak{S}=\boldsymbol{O}_{2}(\mathfrak{T} \mathfrak{O})$. Thus, $\mathfrak{S} \cong \triangleleft \mathfrak{I} \mathfrak{D}$, and $\mathfrak{S} \mathfrak{D} /$ $\mathfrak{S} D(\mathfrak{\Omega})$ is a chief factor of $\mathfrak{T} \Omega$.

Since $\mathfrak{W}_{0} \triangleleft \mathfrak{I}$, it follows that $N_{\mathfrak{x} \mathbb{Q}}\left(\mathfrak{W}_{0}\right)=\mathfrak{I} \mathfrak{\Omega}_{0}$, where $\mathfrak{\Omega}_{0}=\mathfrak{\Omega} \cap$

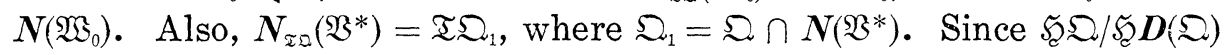
is a chief factor of $\mathfrak{I} \Omega$, it follows that $\Omega_{0}, \Omega_{1} \subseteq D(\Omega)$. This is so since $\mathfrak{S}_{\mathfrak{C}} \mathfrak{D}_{i} \boldsymbol{D}(\mathfrak{D}) \triangleleft \mathfrak{I} \Omega, i=0,1$.

Let $\mathfrak{B}^{G}=\mathfrak{Y}$ be a conjugate of $\mathfrak{B}$ with $\mathfrak{Y} \subseteq \mathfrak{I}, \mathfrak{Y} \varsubsetneqq \mathfrak{S}$. Thus, $\mathfrak{Y}$ acts non trivially on $\mathfrak{S} \cong / \mathfrak{S} D(\Omega)$. Let $\Omega^{*}$ be a subgroup of $\cong$ which is minimal subject to

(i) $\mathfrak{Y}$ normalizes $\mathfrak{S} \mathfrak{C}^{*}$,

(ii) $[\mathfrak{S} \cong *, \mathfrak{Y}] \nsubseteq \mathfrak{S}_{\mathfrak{C}} \boldsymbol{D}(\mathfrak{D})$.

Since $\mathfrak{Y}$ is an elementary 2-group, $\mathfrak{\Omega}^{*}$ is cyclic. Let $\mathbb{Z}=\mathfrak{S} \Omega * \mathfrak{Y}$, and let $\mathbb{\Omega}_{0}=\boldsymbol{O}_{2}(\mathfrak{Q})$. Thus, $\left|\mathfrak{S} \mathfrak{Y}: \mathfrak{\Omega}_{0}\right|=2$. Let $Y$ be an element of $\mathfrak{Y}-\mathfrak{\Omega}_{0}$. Thus, $Y$ inverts some $S_{q}$-subgroup of $\&$, so we assume without loss of generality that $Y$ inverts $\mathfrak{\Omega}^{*}$. Let $\mathfrak{Y}_{0}=\mathfrak{Z}_{0} \cap \mathfrak{Y}$ so that $\mathfrak{Y}_{0}$ is a hyperplane of $\mathfrak{B}^{G}=\mathfrak{Y}$. Let $\mathfrak{R}_{1}=\mathfrak{Y}_{0}^{\mathfrak{Q}} \subseteq \mathfrak{Z}_{0}$. Since $\mathfrak{R}_{1} \subseteq \mathfrak{I}$, and since $\mathfrak{R}_{1}$ is generated by conjugates of $\mathfrak{V}_{0}$, it follows that $\mathfrak{Q}_{1} \subseteq \mathfrak{W}_{1}$. Let $\mathfrak{C}=$ $\boldsymbol{C}_{\mathfrak{x} \Omega}\left(\mathbb{R}_{1}\right)$, so that $\mathbb{\&}$ normalizes $\mathbb{E}$. Since $\mathbb{E}$ centralizes $\mathfrak{Y}_{0}$, it follows that $\mathbb{C} \cong \mathfrak{M}^{a}$. Thus, $\mathbb{C}$ is a subgroup of $\mathfrak{M}^{G}$ which centralizes the hyperplane $\mathfrak{Y}_{0}$ of $\mathfrak{B}^{a}$, and so $\mid \mathfrak{S}:\left(\mathfrak{S} \cap C\left(\mathfrak{B}^{a}\right) \mid \leqq 2\right.$. Thus, $Y$ centralizes a subgroup of $\sqrt{ } \sqrt{ }$ of index 2 . Hence, $\mathfrak{\Omega}^{*}$ centralizes a subgroup of $\sqrt{5}$ of index at most 4 . Now $\mathfrak{W}_{0} \subseteq \mathfrak{C}$, since $\mathfrak{B}_{1} \subseteq \mathfrak{W}_{1}$. Since $\mathfrak{\Omega}^{*} \varsubsetneqq \boldsymbol{D}(\mathfrak{D})$, it follows that $\mathfrak{\Omega}^{*}$ does not centralize $\mathfrak{B}_{0}$. Hence, $\left[\mathfrak{\mathfrak { S }}, \mathfrak{\Omega}^{*}\right]=\mathfrak{S}^{*}$ is a four-group, $q=3$, and $\boldsymbol{D}\left(\mathfrak{\Omega}^{*}\right)$ centralizes $\mathfrak{\mathfrak { E }}$. Let $\mathfrak{S}_{1}^{*}=\left[\mathfrak{S}^{*}, Y\right]$ so that $\left|\mathfrak{S}_{1}^{*}\right|=2, \mathfrak{S}_{1}^{*} \subseteq \mathfrak{B}^{G}$. Let $Q$ be a generator for $\mathfrak{\Omega}^{*}$. Then $\mathfrak{S}_{1}^{* Q} \subseteq \mathfrak{B}^{G Q}$, and $\mathfrak{C}_{1}^{* Q} \subseteq \mathfrak{C}^{*}$. By Lemma $13.8(\mathrm{~b})(\mathrm{i})$, it follows that $\mathfrak{Y}_{0} \subseteq \mathfrak{M}^{\alpha Q}$. By Lemma 13.15, we get $\mathfrak{B}^{G} \subseteq \mathfrak{M}^{G Q}$. Hence, $\left[\mathfrak{B}^{G}, \mathfrak{B}^{G Q}\right]=1$, by Lemma 13.7 applied to $\mathfrak{B}^{G Q}$. In particular, $\left[Y, Y^{Q}\right]=1$. This is not the case, since $\left[Y, Y^{Q}\right]=Q^{2}$. The proof is complete.

Lemma 13.17. One of the following holds:

(a) $|\mathfrak{B}| \leqq 4$. 
(b) There is $V$ in $\mathfrak{S}^{\sharp}$ such that $C(V) \nsubseteq \mathfrak{M}$.

(c) If $G \in \mathfrak{S}$ and $\mathfrak{B}^{G} \cap \mathfrak{M} \neq 1$ then $\mathfrak{B}^{G} \subseteq \mathfrak{M}$.

Proof. Suppose (a) and (b) fail. Choose $G$ in (S) such that $\mathfrak{B}^{G} \cap$ $\mathfrak{M} \neq 1$. Let $\mathfrak{X}=\mathfrak{B}^{G} \cap \mathfrak{M}$. We must show that $\mathfrak{X}=\mathfrak{B}^{G}$. Choose $X$ in $\mathfrak{X}^{\ddagger}$. Since $C(X) \subseteq \mathfrak{M}^{a}$, it follows that $\mathfrak{B} \cap \mathfrak{M}^{a} \neq 1$. Choose $V$ in $\left(\mathfrak{B} \cap \mathfrak{M}^{G}\right)^{\sharp}$. Since $\left|\mathfrak{B}^{G}\right| \geqq 8$, it follows that $\mathfrak{B}^{G} \cap C(V)$ is not cyclic. $\boldsymbol{C}(V) \subseteq \mathfrak{M}$, it follows that $\mathfrak{X}$ is non cyclic. Let $\mathfrak{X}_{1}, \mathfrak{X}_{2}$ be distinct subgroups of $\mathfrak{X}$ of order 2 , and let $\mathfrak{X}_{i}=\left\langle X_{i}\right\rangle, i=1,2$. Let $X_{3}=X_{1} X_{2}$. For each $i=1,2$, 3, let $\mathfrak{B}_{i}=\mathfrak{B} \cap \boldsymbol{C}\left(X_{i}\right)$. Thus, $\mathfrak{B}_{i} \subseteq \mathfrak{M}^{G}$. Suppose by way of contradiction that $\mathfrak{X} \neq \mathfrak{B}^{G}$. Let $\mathfrak{X}_{0}=\mathfrak{X} \cap \boldsymbol{C}(\mathfrak{B})$. Suppose $\mathfrak{X}_{0} \neq 1$. Then $\boldsymbol{C}\left(\mathfrak{X}_{0}\right) \subseteq \mathfrak{M}^{a}$, so $\mathfrak{B} \subseteq \mathfrak{M}^{G}$. By Lemma 13.7 , we get $\left[\mathfrak{B}, \mathfrak{S}^{G}\right]=1$, so $\mathfrak{B}^{G} \subseteq \mathfrak{M}$, against $\mathfrak{X} \subset \mathfrak{B}^{G}$. Hence, $\mathfrak{X}_{0}=1$. Since $\left[\mathfrak{X}_{j}, \mathfrak{B}_{i}\right] \subseteq \mathfrak{B} \cap \mathfrak{B}^{G}$, it follows that $\left[\mathfrak{X}_{j}, \mathfrak{B}_{i}\right]=1,1 \leqq i, j \leqq 3$. This is clearly impossible since $\mathfrak{X}_{1} \mathfrak{X}_{2}$ is faithfully represented on $\boldsymbol{O}_{2}(\mathfrak{M} / \boldsymbol{C}(\mathfrak{B}))$. The proof is complete.

LeMma 13.18. One of the following holds:

(a) $|\mathfrak{B}| \leqq 4$.

(b) $\mathfrak{T} \in \mathscr{L}^{*}$ (SB).

Proof. Suppose false. Let $\mathfrak{S}$ be a solvable subgroup of $\mathbb{S}$ which contains $\mathfrak{I}$ and is minimal subject to $\mathfrak{S} \nsubseteq \mathfrak{M}$. Thus, $\mathfrak{F}=\mathfrak{I} \mathfrak{S}$ where $\mathfrak{O}$ is a $q$-group for some odd prime $q$. Let $\mathfrak{S}_{0}=\boldsymbol{O}_{2}(\mathfrak{S})$. Minimality of $\mathfrak{Q}$ yields $\mathfrak{F}_{0} \mathfrak{S} \triangleleft \mathfrak{S}_{c}$, and also implies that $\boldsymbol{D}(\mathfrak{\Omega})=\mathfrak{Q} \cap \mathfrak{M}$, while $\mathfrak{S}_{0} \cong / \mathfrak{S}_{0} D(\cong)$ is a chief factor of $\mathfrak{S}_{2}$.

Suppose $\mathfrak{B}^{*} \nsubseteq \mathfrak{S}_{0}$. Let $\mathfrak{B}^{G}=\mathfrak{Y}$ be a conjugate of $\mathfrak{B}^{3}$ such that $\mathfrak{Y} \subseteq \mathfrak{I}, \mathfrak{Y} \nsubseteq \mathfrak{F}_{0}$. Let $\mathfrak{Z}^{*}$ be a subgroup of $\cong$ which is minimal subject to

(i) $\mathfrak{Y}$ normalizes $\mathfrak{S}_{0} \mathfrak{D}^{*}$,

(ii) $\left[\mathfrak{S}_{0} \mathfrak{\Omega}^{*}, \mathfrak{V}\right] \equiv \mathfrak{S}_{0} D(\mathfrak{D})$.

Since $\mathfrak{Y}$ is an elementary 2-group, $\mathfrak{Z}^{*}$ is cyclic. Let $\mathfrak{Y}_{0}=\mathfrak{Y} \cap \boldsymbol{O}_{2}(\mathfrak{Q})$, where $\mathfrak{Z}=\mathfrak{K}_{0} \mathfrak{\Omega} * \mathfrak{Y}$. Thus, $\mathfrak{Y}_{0}$ is a hyperplane of $\mathfrak{Y}$. Let $Q$ be a generator for $\mathfrak{Z}^{*}$. Then $\mathfrak{Y}_{0}^{2} \subseteq \boldsymbol{O}_{2}(\mathfrak{Q}) \subseteq \mathfrak{T} \subseteq \mathfrak{M}$, so by Lemma 13.15 , we get $\mathfrak{Y}^{2} \leqq \mathfrak{M}$. Thus, $\left\langle\mathfrak{Y}, \mathfrak{Y}^{Q}\right\rangle \subseteq \mathfrak{M}$. Since $\left\langle\mathfrak{Y}, \mathfrak{Y}^{Q}\right\rangle$ contains a $S_{q}$-subgroup of $\mathfrak{L}$, we have $\mathfrak{Q} \subseteq \mathfrak{M}$. This violates $\boldsymbol{D}(\mathfrak{Q})=\mathfrak{Q} \cap \mathfrak{M}$. We conclude that $\mathfrak{B}^{*} \subseteq \mathfrak{S}_{0}$. Hence, $\mathfrak{B}^{*} \triangleleft \mathfrak{\mathcal { C }}$.

Since $\mathfrak{S} \nsubseteq \mathfrak{M}$, and since $\mathfrak{B}^{*} \triangleleft \mathfrak{S}$, we conclude that $N\left(\mathfrak{B}^{*}\right) \nsubseteq \mathfrak{M}$. By Lemma 13.8, we get $C(V) \subseteq \mathfrak{M}$ for all $V$ in $\mathfrak{B}^{\sharp}$. Thus, (c) of Lemma 13.16 holds. We conclude that $N\left(\mathfrak{W}_{0}\right)$ contains an element of $\mathscr{A}(p)$ for some $p$ in $\sigma$, so $N\left(\mathfrak{W}_{0}\right) \subseteq \mathfrak{M}$. Since $\mathfrak{W}_{0}$ char $\mathfrak{W}_{1}$, we conclude that $\mathfrak{W}_{1} \nsubseteq \mathfrak{S}_{0}$. By definition of $\mathfrak{W}_{1}$, there is an element $G$ in (S) such that $\mathfrak{B}^{G} \cap \mathfrak{W}_{1}$ is of index at most 2 in $\mathfrak{B}^{G}$, and such that $\mathfrak{B}^{G} \cap \mathfrak{W}_{1} \nsubseteq \mathfrak{S}_{2}$. Let $\mathfrak{Y}=\mathfrak{B}^{G} \cap \mathfrak{W}_{1}$. Since $\mathfrak{B}^{*} \subseteq \mathfrak{F}_{0}$, it follows that $\left|\mathfrak{B}^{G}: \mathfrak{Y}\right|=2$.

Let $\mathfrak{\Omega}^{*}$ be a subgroup of $\mathfrak{Q}$ which is minimal subject to

(i) $\mathfrak{Y}$ normalizes $\mathfrak{Q} * \mathfrak{F}_{\mathfrak{c}}$, 
(ii) $\left[\mathfrak{S}_{0} \cong *, \mathfrak{V}\right] \nsubseteq \mathfrak{S}_{0} D(\mathfrak{D})$.

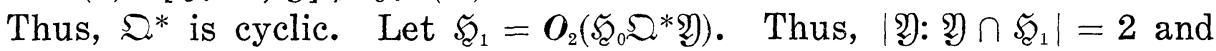
$\mathfrak{S}_{1} \subseteq \mathfrak{T}$. Since $|\mathfrak{B}| \geqq 8$, we have $\mathfrak{Y} \cap \mathfrak{S}_{1} \neq 1$. Let $Q$ be a generator for $\mathfrak{\Omega}^{*}$. Then $\left(\mathfrak{Y} \cap \mathfrak{S}_{1}\right)^{Q} \neq 1$, and $\left(\mathfrak{Y} \cap \mathfrak{S}_{1}\right)^{Q} \subseteq \mathfrak{S}_{1} \subseteq \mathfrak{I} \subseteq \mathfrak{M}$. Thus, by Lemma 13.17, we get $\mathfrak{B}^{a Q} \subseteq \mathfrak{M}$. In particular, $\left\langle\mathfrak{Y}, \mathfrak{Y}^{Q}\right\rangle \subseteq \mathfrak{M}$. Since

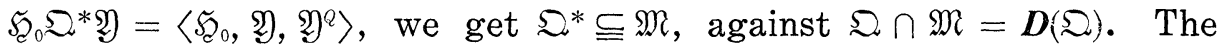
proof is complete.

We can at last obtain some important information about $\mathfrak{B}$.

Lemma $13.19 .|\mathfrak{B}| \leqq 4$ 。

Proof. Suppose $|\mathfrak{B}| \geqq 8$. Then by Lemma 13.18, we get $N\left(\mathfrak{S}^{*}\right) \subseteq \mathfrak{M}$. Let $\mathscr{V}$ be the set of 2 -subgroups of $\mathfrak{M}$ which contain $\mathfrak{B}$.

We will show that $\mathscr{V} \subseteq \mathscr{C}^{*}(\mathbb{S})$. Suppose false, and $\mathfrak{X}$ is an element of $\mathscr{Y}-\mathscr{C}^{*}(\mathbb{S})$ of maximal order. Let $\mathfrak{S}$ be a solvable subgroup of (s) of minimal order subject to

(i) $\mathfrak{U} \subseteq \mathfrak{S}$,

(ii) $\mathfrak{S} \nsubseteq \mathfrak{M}$.

By maximality of $|\mathfrak{A}|, \mathfrak{A}$ is a $S_{2}$-subgroup of $\mathfrak{S}$. By minimality of $\mathfrak{F}, \mathfrak{S}=\mathfrak{U} \Omega$ where $\mathfrak{D}$ is a $q$-group for some odd prime $q$.

Let $\mathfrak{U} \in \mathscr{Q} 6(\mathfrak{I}), \mathfrak{H} \subseteq \mathfrak{U} ; \mathfrak{U}$ exists since $\mathfrak{B} \triangleleft \mathfrak{I}, \Omega_{1}(\boldsymbol{Z}(\mathfrak{T})) \subseteq \mathfrak{B}$ and $|\mathfrak{B}| \geqq 4$. Then $\mathfrak{U}$ centralizes $O_{q}(\mathfrak{S})$, so $O_{q}(\mathfrak{S}) \subseteq N(\mathfrak{U})$. Since $\mathfrak{I} \subseteq N(\mathfrak{U})$, we get $O_{q}(\mathfrak{S}) \subseteq \mathfrak{M}$, by Lemma 13.18. By minimality of $\mathfrak{S}$, we get

$$
\boldsymbol{O}_{q, 2}(\mathfrak{S})=\boldsymbol{O}_{q}(\mathfrak{S}) \times \boldsymbol{O}_{2}(\mathfrak{S T}) .
$$

Let $\mathfrak{B S}^{*}=V\left(\operatorname{ccl}_{\Theta}(\mathfrak{B}) ; \mathfrak{X}\right)$. If $\mathfrak{W}^{*} \subseteq \boldsymbol{O}_{2}(\mathfrak{S})$, then maximality of $\mathfrak{2}$ forces $\mathfrak{Z}$ to be a $S_{2}$-subgroup of (S). This violates Lemma 13.18 . Hence, $\mathfrak{W}^{*} \nsubseteq \boldsymbol{O}_{2}\left(\mathfrak{F}_{2}\right)$.

Let $\mathfrak{B}^{G}=\mathfrak{Y}$ be a conjugate of $\mathfrak{B}$ such that $\mathfrak{Y} \leqq \mathfrak{A}, \mathfrak{V} \nsubseteq \boldsymbol{O}_{2}(\mathfrak{S})$. By minimality of $\mathfrak{S}$, we have $\boldsymbol{O}_{2}(\mathfrak{S}) \cong \triangleleft \mathfrak{S}, \mathfrak{Z} \cap \mathfrak{M}=\boldsymbol{D}(\mathfrak{\Omega})$. Let $\mathfrak{\Omega}^{*}$ be a subgroup of $\mathfrak{Q}$ which is minimal subject to

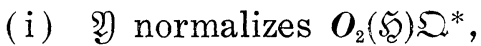

(ii) $\left[O_{2}\left(\mathfrak{S}_{\mathcal{E}}\right) \Omega^{*}, \mathfrak{Y}\right] \nsubseteq \mathrm{O}_{2}(\mathfrak{S}) \mathrm{D}_{(\mathfrak{O})}$.

Thus, $\mathfrak{Z}^{*}$ is cyclic and $\mathfrak{Y}_{0}=\mathfrak{Y} \cap \boldsymbol{O}_{2}\left(\mathfrak{Q}_{1}\right)$ is of index 2 in $\mathfrak{Y}$, where $\mathfrak{Z}_{1}=$ $\mathrm{O}_{2}(\mathfrak{S}) \cong * \mathfrak{Y}$. Let $Q$ be a generator for $\mathfrak{\Omega}^{*}$. Then $\mathfrak{Y}_{0}^{\mathbb{Q}} \subseteq \mathfrak{I}$, so by Lemma 13.15 , we get $\mathfrak{Y}^{2} \cong \mathfrak{M}$. Since $\mathfrak{Q}_{1}=\left\langle\boldsymbol{O}_{2}(\mathfrak{F}), \mathfrak{Y}, \mathfrak{Y}^{Q}\right\rangle$, we get $\mathfrak{Z}^{*} \subseteq \mathfrak{M}$, against $\mathfrak{\Omega} \cap \mathfrak{M}=\boldsymbol{D}(\mathfrak{\Omega})$. This contradiction shows that $\mathscr{V} \subseteq \mathscr{C}^{*}(\mathbb{S})$.

We next show that if $\mathfrak{B}^{G} \cap \mathfrak{M} \neq 1$, then $G \in \mathfrak{M}$. Namely, if $V \in \mathfrak{B}^{\sharp}$, then $C(V) \subseteq \mathfrak{M}$, since $\mathfrak{B} \in \mathscr{Y}$. Hence, (c) of Lemma 13.17 holds. Since $\mathfrak{B}^{G} \cap \mathfrak{M} \neq 1$, we have $\mathfrak{B}^{G} \subseteq \mathfrak{M}$. Since $\mathfrak{S}^{G} \in \mathscr{M}^{*}(\mathbb{S})$, we have $\mathfrak{M}=\mathfrak{M}^{G}$, $G \in \mathfrak{M}$.

Let $I$ be an involution of $\mathfrak{M}$. We will show that $C(I) \subseteq \mathfrak{M}$. Let $\mathfrak{Z}$ be a $S_{2}$-subgroup of $C_{\mathfrak{n}}(I)$, and let $\mathfrak{2} *$ be a $S_{2}$-subgroup of $C(I)$ which contains $\mathfrak{A}$. Suppose $\mathfrak{U} \subset \mathfrak{X}^{*}$. Choose $A \in \mathfrak{U}^{*} \cap N(\mathfrak{U})-\mathfrak{X}$. Then 
$1 \neq \mathfrak{B} \cap \mathfrak{U} \subseteq \mathfrak{M} \cap \mathfrak{M}^{A}$, so $\mathfrak{M}=\mathfrak{M}^{A}, A \in \mathfrak{M}$. Thus, $\mathfrak{X}=\mathfrak{2} *$. By Lemma 13.1, $O_{2^{\prime}}(C(I))=1$. Let $\mathfrak{A}_{0}=O_{2}(C(I))$. It suffices to show that $N\left(\mathfrak{U}_{0}\right) \subseteq$ M. Now $\mathfrak{A}_{0} \cap \mathfrak{B} \neq 1$, since $Z\left(\mathfrak{A}_{0}\right)=C_{\mathfrak{r}}\left(\mathfrak{U}_{0}\right)$. Hence, if $N \in N\left(\mathfrak{N}_{0}\right)$, then $\mathfrak{H}_{0} \cap \mathfrak{B} \subseteq \mathfrak{M}^{N}$, so that $\mathfrak{M}=\mathfrak{M}^{N}, N \in \mathfrak{M}$. This completes a proof that $C(I) \subseteq \mathfrak{M}$ for every involution $I$ of $\mathfrak{M}$. Now Lemma 13.9 and Theorem 13.2 are in conflict. The proof is complete.

Lemma 13.20. Suppose $\mathfrak{R} \in \mathscr{U} \mathscr{S}$ (S) and the following hold:

( a ) $O_{2},(\mathfrak{R})=1$,

(b) $\mathfrak{R} \cap \mathfrak{M}$ contains a $S_{2}$-subgroup of $\mathfrak{R}$.

(c) $\mathfrak{R} \neq \mathfrak{M}$.

Then $\mathfrak{N}$ does not contain an elementary subgroup of order $p^{3}$ for any odd prime $p$.

Proof. Suppose false. Let $p$ be an odd prime such that $\mathfrak{R}$ contains an elementary subgroup $\mathfrak{F}$ of order $p^{3}$. Hence, $\mathfrak{R}=\boldsymbol{M}(\mathfrak{F})$. Let $\mathfrak{V}_{0}=\Omega_{1}\left(\boldsymbol{R}_{2}(\mathfrak{N})\right)$. By Lemma $13.8(\mathrm{c})$ applied to $\mathfrak{R}, \mathfrak{R}$ contains a $S_{2}$ subgroup of $\mathbb{B}$, so by (b), $\mathfrak{R} \cap \mathfrak{M}$ contains a $S_{2}$-subgroup $\mathfrak{I}^{*}$ of $\mathbb{B}$. By Lemma 13.19 applied to $\mathfrak{R}$, we get $\left|\mathfrak{B}_{0}\right| \leqq 4$. By Lemma 5.7, $\mathfrak{B} \cap \mathfrak{B}_{0} \supseteqq \Omega_{1}\left(Z\left(\mathfrak{I}^{*}\right)\right)$. Choose $Z$ in $\mathfrak{B} \cap \mathfrak{B}_{0}^{\sharp}$. Since $C_{\mathfrak{n}}(Z)$ contains an element of $\mathscr{A}(q)$ for all $q$ in $\sigma$, we get $C(Z) \subseteq \mathfrak{M}$. Since $C_{\Im}(Z)$ is non cyclic, we also get $C_{\Im}(Z) \in \mathscr{C}^{*}(\mathbb{S})$. Hence, $\mathfrak{M}=\mathfrak{N}$, against (c). The proof is complete.

Let $\tau$ be the set of all odd primes $q \in \pi(\mathfrak{M})-\sigma$ such that $C_{\mathfrak{m}}(Q) \in$ $\mathscr{C}^{*}(\mathbb{S})$ for all elements $Q$ of $\mathfrak{M}$ of order $q$.

Lemma 13.21. Suppose $q \in \tau$ and $\cong$ is a $S_{q}$-subgroup of $\mathfrak{M}$. Then one of the following holds:

(a) $\mathfrak{Q}$ is cyclic, $q \in \pi_{1}$, and $N(\mathfrak{D}) \subseteq \mathfrak{M}$.

(b) $q \in \pi_{2}$ and $\Omega_{0} \in \mathscr{l}^{*}$ (S) for every non cyclic $q$-subgroup $\Omega_{0}$ of (B).

Proof. Suppose $\cong$ is cyclic. Choose $Q$ in $\mathscr{Q}$ of order $q$. Then

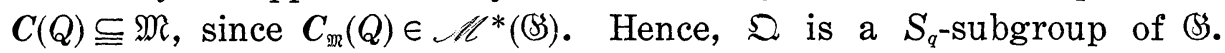
Choose $N \in N(\Omega)$. Then $\langle C(\Omega), N\rangle$ is solvable, so $\langle C(\Omega), N\rangle \subseteq \mathfrak{M}$, and (a) holds.

Suppose $\mathfrak{Q}$ is non cyclic. Let $\mathfrak{Q}^{*}$ be a $S_{q}$-subgroup of (5) which contains $\Omega$. Choose $Q$ in $\mathfrak{Q}$ of order $q, Z$ in $Z\left(\Omega^{*}\right)$ of order $q$. Since $\boldsymbol{C}(Q) \subseteq \mathfrak{M}$, we have $Z \in \mathfrak{M}$. Since $\boldsymbol{C}(Z) \subseteq \mathfrak{M}$, we have $\mathfrak{Z}=\mathfrak{\Omega}^{*}$. Since $q \notin \sigma$, it follows that $q \in \pi_{2}$. Let $\tilde{\mathfrak{D}}$ be any non cyclic $q$-subgroup of (S. Then $\tilde{\mathfrak{a}} \subseteq \mathfrak{M}^{G}$ for some $G$ in $\mathbb{S}$. Let $\widehat{\mathfrak{D}}$ be a $S_{q}$-subgroup of (S) which contains $\tilde{\mathfrak{Q}}$. Then $\hat{\mathfrak{Q}} \subseteq \mathfrak{M}^{G}$, as above. Let $\mathfrak{R}$ be a subgroup of $\widetilde{\mathfrak{Q}}$ of type $(q, q)$. Then $C(R) \subseteq \mathfrak{M}^{G}$ for all $R$ in $\mathfrak{R}^{\sharp}$, so every element of $И\left(\mathfrak{R} ; q^{\prime}\right)$ is contained in $\mathfrak{M}^{G}$. Let $\tilde{\mathfrak{D}} \subseteq \mathfrak{S} \in \mathscr{S} \mathscr{O} \mathscr{L}(\mathbb{S})$. Thus $\boldsymbol{O}_{q^{\prime}}(\mathfrak{S}) \subseteq$ 
$\mathfrak{M}^{G}$, and $\mathfrak{S} \cap \mathfrak{M}^{G}$ contains a $S_{q}$-subgroup $\mathscr{S}_{q}$ of $\mathfrak{S}$ with $\tilde{\mathfrak{D}} \subseteq \mathscr{S}_{q} \cdot$ Let

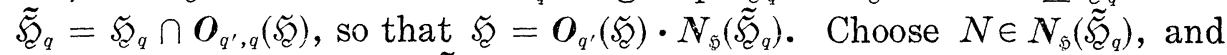
let $H$ be an element of $\tilde{\mathcal{F}}_{q}$ of order $q$. Then $C(H) \cong \mathfrak{M}^{a}, C\left(H^{N}\right) \cong \mathfrak{M}^{a}$, and $C(H) \in \mathscr{C}^{*}(\mathbb{S})$. Hence, $\mathfrak{M}^{G}=\mathfrak{M}^{G N}, N \in \mathfrak{M}^{G}$, and so $\tilde{\mathfrak{D}} \in \mathscr{C l}^{*}(\mathbb{B})$. The proof is complete.

Lemma 13.22. Suppose $q \in \pi(\mathfrak{M})-\sigma, q$ is an odd prime, and for some $p$ in $\sigma$, an $S_{p}$-subgroup $\mathfrak{P}$ of $\mathfrak{M}$ does not centralize every element of $\boldsymbol{V}_{\mathfrak{v}}(\mathfrak{P} ; q)$. Then $q \in \pi_{2} \cap \tau$.

Proof. Let $\mathfrak{Q}$ be a $S_{q}$-subgroup of $\mathfrak{M}$ permutable with $\mathfrak{R}$, and let $\mathfrak{\Omega}_{0}=O_{q}(\mathfrak{B} \Omega)$. Thus, $\mathfrak{\Omega}_{0}$ is a $S_{q}$-subgroup of $\boldsymbol{O}_{p^{\prime}}(\mathfrak{M})$ and $\mathfrak{P}$ does not centralize $\mathfrak{Z}_{0}$, as $\mathfrak{\Omega}_{0}$ is a maximal element of $\boldsymbol{U}_{\mathfrak{n}}(\mathfrak{P} ; q)$. Since $\mathfrak{P} \in$ $\mathscr{C l}^{*}(\mathbb{S}), \mathfrak{\Omega}$ is a $S_{q}$-subgroup of $N\left(\mathfrak{\Omega}_{0}\right)$. Since $q \notin \sigma, \mathscr{S} \mathscr{C} \mathscr{N}_{3}(\mathfrak{D})=\varnothing$. Since $\mathfrak{P} \subseteq \mathfrak{M}^{\prime}$, so also $\mathfrak{P} \subseteq N\left(\mathfrak{\Omega}_{0}\right)^{\prime}$, since $\mathfrak{M}=O_{p^{\prime}}(\mathfrak{M}) \cdot N\left(\mathfrak{\Omega}_{0}\right)$. By Lemma 10.6, we have

(i) $\Omega_{1}(\Omega) \subseteq \Omega_{0}$,

(ii) $\mathfrak{\Omega}$ is a $S_{q}$-subgroup of (3),

(iii) $q \in \pi_{2}$.

Let $\mathfrak{W}=\Omega_{1}(\mathfrak{Q}) / \boldsymbol{D}\left(\Omega_{1}(\mathfrak{Q})\right)$, so that $\mathfrak{W}$ is of order $q^{2}$. Since each element of $\mathfrak{P}$ induces a linear transformation of $\mathfrak{B}$ of determinant 1 , it follows that $\mathfrak{B} \cap \boldsymbol{C}\left(\Omega_{1}(\mathfrak{Q})\right)$ is non cyclic. Since $\mathfrak{P} \cap \boldsymbol{C}\left(\Omega_{1}(\mathfrak{\Omega})\right) \triangleleft \mathfrak{B}$, we get $\mathfrak{B} \cap C\left(\Omega_{1}(\mathfrak{\Omega})\right) \in \mathbb{C l}^{*}(\mathbb{S})$. Choose $Q \in \Omega_{1}(\mathfrak{\Omega})^{*}$. Then $\mathbb{C}_{\mathfrak{m}}(Q)$ contains an element of $\mathscr{A}(p)$, so $C_{\mathfrak{m}}(Q) \in \mathscr{C l}^{*}(G)$. This gives $q \in \tau$ and completes the proof.

Lemma 13.23. Suppose $p \in \sigma \cup \tau$ and $\mathbb{Q}$ is $a 2$, p-subgroup of (S). If $\mathbb{\Omega} \cap \mathfrak{M}$ contains a non cyclic p-group, then $\mathbb{Q} \subseteq \mathfrak{M}$.

Proof. If $p \in \tau$, then by Lemma 13.21 (b), we get $\& \cap \mathfrak{M} \in \mathscr{C l}^{*}(\mathbb{B})$ and we are done. Suppose $p \in \sigma$.

We assume without loss of generality that $\mathbb{B}$ is a maximal $2, p$ subgroup of 8 .

By Lemma 6.6 (iii), either $O_{2}(\mathbb{Q})=1$ or $O_{p}(\mathfrak{R})=1$. Let $\mathfrak{S}_{p}$ be a $S_{p}$-subgroup of $\mathcal{Z}$ which contains a $S_{p}$-subgroup $\tilde{\Omega}_{p}$ of $\mathbb{Q} \cap \mathfrak{M}$. By hypothesis, $\tilde{\Omega}_{p}$ is non cyclic. Since $p \in \sigma$, it follows that $N_{\mathfrak{n}}\left(\tilde{\Omega}_{p}\right)$ contains an element of $\mathscr{A}(p)$. Hence, $N_{\mathfrak{n}}\left(\tilde{\Omega}_{p}\right) \in \mathscr{C l}^{*}(\mathbb{S})$. This implies that $\tilde{\mathfrak{R}}_{p}=\mathfrak{R}_{p}$. We may assume that $\mathfrak{R}_{p}$ contains no element of $\mathscr{A}(p)$. Thus, $\mathbb{R}_{p}$ contains a cyclic subgroup of index $p$, so that the $p$-length

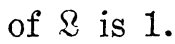

If $O_{2}(\mathbb{R})=1$, then $\mathbb{R}_{p} \triangleleft \mathbb{R}$. As $\&$ is a maximal $2, p$-subgroup of (S), we get that $\mathfrak{R}_{p}$ is a $S_{p}$-subgroup of (3), against $p \in \sigma$. Hence, $O_{2}(\mathbb{R}) \neq 1$, so that $O_{p}(\mathbb{R})=1$.

Let $\Re=O_{2}(\mathbb{R}), \mathfrak{B}=\Omega_{1}\left(\mathfrak{Q}_{p}\right)$. Since $\mathfrak{B} \notin \mathscr{A}(p)$, there is an element 
$B_{0}$ of $\mathfrak{B}$ such that $C\left(B_{0}\right)$ does not contain an elementary subgroup of order $p^{3}$. Thus, if $\mathfrak{F}$ is a $S_{p}$-subgroup of $C\left(B_{0}\right)$ which contains $\mathfrak{B}$, then $\mathfrak{B}=\Omega_{1}(\mathfrak{P})$. By Lemma 5.25, if $\mathfrak{S} \in \mathscr{S} \mathcal{O} \mathscr{C}(\mathfrak{S}), \mathfrak{B} \subseteq \mathfrak{S}$, then every element of $\boldsymbol{И}_{\mathfrak{\Phi}}\left(\mathfrak{B} ; p^{\prime}\right)$ is in $\boldsymbol{O}_{p^{\prime}}(\mathfrak{S})$. Let $\mathfrak{B}^{*}$ be a $S_{p}$-subgroup of (5) which contains $\mathfrak{P}_{\text {. }}$ Then $\mathfrak{P}=\left\langle B_{0}\right\rangle \times \mathfrak{P}_{0}$, where $\mathfrak{P}_{0}$ is cyclic and $\mathfrak{P}_{0} \supseteqq$ $\Omega_{1}\left(\boldsymbol{Z}\left(\mathfrak{S}^{*}\right)\right)$. It follows that $\boldsymbol{N}_{\mathfrak{\beta}^{*}}(\mathfrak{P})$ permutes transitively the subgroups of $\mathfrak{B}$ of order $p$ distinct from $\Omega_{1}\left(\boldsymbol{Z}\left(\mathfrak{S}^{*}\right)\right)$. Thus, if $X \in \mathfrak{B}-\Omega_{1}\left(\boldsymbol{Z}\left(\mathfrak{S}^{*}\right)\right)$, then $\mathfrak{P}$ is a $S_{p}$-subgroup of $C(X)$.

Since $\mathfrak{B}$ is faithfully represented on $\Re, \Omega_{1}\left(\mathbb{Z}\left(\mathfrak{P}^{*}\right)\right)$ does not centralize ${ }^{\circ}$. Since $\Re=\left\langle C_{\Re}(B) \mid B \in \mathfrak{B}^{\ddagger}\right\rangle$, it follows that for some $X$ in $\mathfrak{B}-$ $\Omega_{1}\left(\boldsymbol{Z}\left(\mathfrak{P}^{*}\right)\right), \boldsymbol{C}_{\Omega}(X) \nsubseteq \boldsymbol{C}\left(\Omega_{1}\left(\boldsymbol{Z}\left(\mathfrak{S}^{*}\right)\right)\right)$. Thus, $\boldsymbol{C}_{\AA}(X) \subseteq \boldsymbol{O}_{p^{\prime}}(\mathfrak{S})$, where $\subseteq=\boldsymbol{C}(X)$. Hence, 1 is not the only element of $U(\mathfrak{P} ; 2)$. Let $\Re_{0}$ be a maximal element of $\boldsymbol{U}(\mathfrak{B} ; 2)$ which contains a $S_{2}$-subgroup of $\boldsymbol{O}_{p},(\mathcal{S})$, and let $\Re_{1}$ be a maximal element of $U^{(\mathfrak{B} ; 2)}$ which contains $\Omega_{0}$. Since $\Omega_{0}$ is a $S_{2}$-subgroup of $\boldsymbol{O}_{p^{\prime}}\left(N\left(\Re_{0}\right)\right)$ and since $\boldsymbol{N}_{\Omega_{1}}\left(\Re_{0}\right) \subseteq \boldsymbol{O}_{p^{\prime}}\left(N\left(\Re_{0}\right)\right)$, we get that $\Re_{0}=\Re_{1}$. Hence, $\mathfrak{P}$ normalizes some maximal element $\Omega_{0}$ of $\boldsymbol{U}(\mathfrak{B} ; 2)$ which is not centralized by $\Omega_{1}\left(Z\left(\mathfrak{P}^{*}\right)\right)$.

Let $\widehat{\mathfrak{B}}=\left\{X \mid X \in \mathfrak{B}^{\sharp}, C_{\mathfrak{\Omega}}(X) \neq 1\right\}, \hat{\mathfrak{B}}_{0}=\left\{X \mid X \in \mathfrak{B}^{\sharp}, C_{\mathfrak{S}_{0}}(X) \neq 1\right\}$. Thus $\widehat{\mathfrak{B}} \nsubseteq \Omega_{1}\left(\boldsymbol{Z}\left(\mathfrak{P}^{*}\right)\right), \hat{\mathfrak{B}}_{0} \nsubseteq \Omega_{1}\left(\boldsymbol{Z}\left(\mathfrak{P}^{*}\right)\right)$. Since $\boldsymbol{N}_{\mathfrak{F}} *(\mathfrak{P})$ permutes transitively the subgroups of $\mathfrak{B}$ of order $p$ distinct from $\Omega_{1}\left(Z\left(\mathfrak{P}^{*}\right)\right)$, we can choose $P$ in $\boldsymbol{N}_{\Re^{*}}(\mathfrak{P})$ such that $\widehat{\mathfrak{B}}^{P} \cap \widehat{\mathfrak{B}}_{0} \neq \varnothing$. By Lemma 6.3, $\mathscr{\Re}^{P}$ and $\Re_{0}$ are conjugate by an element of $\boldsymbol{C}(\mathfrak{B})$. Hence, $N(\Omega)$ contains an $S_{p}$-subgroup of $\boldsymbol{C}(\mathfrak{B})$. Thus, we may choose $A$ in $\boldsymbol{C}^{\prime}(\mathfrak{B})$ such that $\mathfrak{\Omega}_{p} \supseteqq \mathfrak{P}^{A}$. Replacing $\mathfrak{P}^{*}$ by $\mathfrak{S}^{* A}$, we may assume that $\mathfrak{Q}_{p} \supseteqq \mathfrak{P}$. If $\mathfrak{B}_{p} \supset \mathfrak{P}$, then $\mathfrak{R}_{p}$ is a $S_{p}$ subgroup of $N\left(\mathfrak{S}_{\mathcal{S}}\right)$, so contains an element of $\mathscr{A}(p)$. This is not the case, so $\mathfrak{Z}_{p}=\mathfrak{P}$.

Suppose $C_{\Omega}\left(\Omega_{1}\left(\mathbb{Z}\left(\mathfrak{P}^{*}\right)\right)\right) \neq 1$. Since $N(\mathfrak{P}) \subseteq N\left(\Omega_{1}\left(\mathbb{Z}\left(\mathfrak{P}^{*}\right)\right)\right)$, it follows from Lemma 6.3 that $\mathfrak{P}$ is not a $S_{p}$-subgroup of $N(\Re)$. Since $\&$ is a $S_{2, p}$-subgroup of $N(\Re)$, this is impossible. Hence, $C_{\Omega}\left(\Omega_{1}\left(Z\left(\mathfrak{Z}^{*}\right)\right)\right)=1$. Hence, $\Re=\left\langle\boldsymbol{C}_{\Omega}\left(\mathfrak{B}^{*}\right)\right| \mathfrak{B}^{*}$ is of order $\left.p, \mathfrak{B}^{*} \subset \mathfrak{B}, \mathfrak{B}^{*} \neq \Omega_{1}\left(\boldsymbol{Z}\left(\mathfrak{B}^{*}\right)\right)\right\rangle$. Since $\mathfrak{B}$ acts faithfully on $\Re$, there are $\mathfrak{B}_{1}, \mathfrak{B}_{2}, \mathfrak{B}_{1} \neq \mathfrak{B}_{2}$ of order $p$ in $\mathfrak{B}$ such that $C_{\Omega}\left(\mathfrak{B}_{i}\right) \neq 1, i=1,2$. We can then choose $P$ in $N_{\mathfrak{P}^{*}}(\mathfrak{P})$ such that $\mathfrak{V}_{1}^{P}=\mathfrak{B}_{2}$. Hence, $C_{\Re}\left(\mathfrak{B}_{2}\right) \neq 1, C_{\Re^{P}}\left(\mathfrak{B}_{2}\right) \neq 1$. Hence, $\Re=\Re^{P C}$ for some $C$ in $C(\mathfrak{P})$. Hence, $\mathfrak{B}$ is not a $S_{p}$-subgroup of $N(\Re)$. This contradiction completes the proof.

For each $p$ in $\sigma$, let $\hat{\mathfrak{M}}_{p}=\left\{P \mid P \in \mathfrak{M}, P\right.$ is of order $p, C_{\mathfrak{g}}(P)$ contains an elementary subgroup of order $\left.p^{3}\right\}$, and for each $p$ in $\tau$, let $\hat{\mathfrak{M}}_{p}=\{P \mid P \in \mathfrak{M}, P$ is of order $p\}$.

Lemma 13.24. Suppose $p \in \sigma \cup \tau$, and $\mathfrak{X}_{0}$ is an elementary subgroup of $\mathfrak{M}$ of order 8. Then $\left\langle\mathfrak{X}_{0}, P_{0}\right\rangle \in \mathscr{C l}^{*}(\mathbb{S})$ for all $P_{0} \in \hat{\mathfrak{M}}_{p}$.

Proof. Let $\&$ be a $S_{2, p}$-subgroup of $\left\langle\mathfrak{X}_{0}, P_{0}\right\rangle$, and suppose by way of contradiction that $\left\langle\mathfrak{x}_{0}, P_{0}\right\rangle \in \mathscr{C}^{*}(\mathcal{S})$. Since $\mathcal{Z} \subseteq\left\langle\mathfrak{x}_{0}, P_{0}\right\rangle$, it follows 
that $\mathfrak{L} \notin \mathscr{C}^{*}(\mathbb{S})$. In addition, $\mathbb{L}$ contains an elementary subgroup $\mathfrak{X}$ of order 8 and an element $P$ of $\hat{\mathfrak{M}}_{p}$.

Let $\mathscr{N}=\{\mathfrak{N} \mid \mathfrak{R} \in \mathscr{S} \bigcirc \mathscr{L}(\mathbb{S}), \mathfrak{R} \subseteq \mathfrak{R} \nsubseteq \mathfrak{M}\}$, so that $\mathscr{N} \neq \varnothing$. Among all elements of $\mathscr{N}$, let $\mathfrak{N}$ be chosen so that $|\mathfrak{N} \cap \mathfrak{M}|_{2}$ is maximal, and with this restriction, $|\mathfrak{N}|$ is minimal.

Case $1 . \quad \mathfrak{R}$ is a $2, p$-group.

Let $\tilde{\mathfrak{N}}_{2}$ be a $S_{2}$-subgroup of $\mathfrak{M} \cap \mathfrak{R}$ and let $\mathfrak{R}_{2}, \mathfrak{R}_{p}$ be a Sylow system of $\mathfrak{R}$ with $\tilde{\mathfrak{N}}_{2} \subseteq \mathfrak{N}_{2}, P \in \mathfrak{N}_{p}$. If $\mathfrak{N}_{p}$ is non cyclic, so is $\mathfrak{N}_{p} \cap \boldsymbol{C}(P)$, so by Lemma 13.23 , we get $\mathfrak{R} \subseteq \mathfrak{M}$. This is not the case, so $\mathfrak{R}_{p}$ is cyclic. In particular, $S_{p}$-subgroups of $\left\langle\mathfrak{X}_{0}, P_{0}\right\rangle$ are cyclic. Since $P_{0}$ is of order $p, S_{p}$-subgroups of $\left\langle\mathfrak{X}_{0}, P_{0}\right\rangle$ are of order $p$. Hence, $\mathfrak{N}_{p}=\langle P\rangle$, by minimality of $|\mathfrak{N}|$.

Let $\Re=\boldsymbol{O}_{2}(\mathfrak{R})$. Thus, $\mathfrak{R}=\mathfrak{\Re} \cdot \boldsymbol{N}_{\mathfrak{R}}\left(\mathfrak{R}_{p}\right)$, since $\left|\mathfrak{R}_{p}\right|=p$. Also, $\mathfrak{R}_{2} / \Re$ is faithfully represented on $\Re_{p} / \Re$, so $\mathfrak{R}_{2} / \Re$ is cyclic. Since $\mathfrak{R}_{2} \nsubseteq \mathfrak{M}$ and $N\left(\mathfrak{R}_{p}\right) \subseteq \mathfrak{M}$, it follows that $\Re \nsubseteq \mathfrak{M}$. Let $\Re_{0}=\Re \cap \mathfrak{M} \subset \Re$. Since $\mathfrak{X}$ is non cyclic, and since $\mathfrak{R} \supseteq \mathbb{Q} \supseteq \mathfrak{X}$, it follows that $\Re_{0} \neq 1$. Let $\subseteq$ be a $S_{2, p}$-subgroup of $N\left(\Re_{0}\right)$ which contains $N_{\Re}\left(\Omega_{0}\right)$. Since $N_{\Omega}\left(\Re_{0}\right) \nsubseteq \mathfrak{M}$, we have $\subseteq \nsubseteq \mathfrak{M}$. By Lemma 13.23, $S_{p}$-subgroups of $\mathfrak{S}$ are cyclic. Let $\widetilde{S}_{1}$ be a $S_{2, p}$-subgroup of $N\left(\Re_{0}\right)$ which contains $\tilde{\Re}_{2} \Re_{p}$ and also contains a $S_{2, p}$-subgroup of $N_{\mathfrak{M}}\left(\Re_{0}\right)$. Since $\mathfrak{R}_{p} \subseteq \mathfrak{S} \cap \mathfrak{S}_{1}$, there is $A$ in $N\left(\Re_{0}\right) \cap N\left(\mathfrak{R}_{p}\right)$ such that $\subseteq=\mathfrak{S}_{1}^{A}$. Since $A \in N\left(\mathfrak{R}_{p}\right) \subseteq \mathfrak{M}$, we have $\mathfrak{S}_{1} \nsubseteq \mathfrak{M}$. By maximality of $|\mathfrak{R} \cap \mathfrak{M}|_{2}$, it follows that $\left|\mathfrak{S}_{1} \cap \mathfrak{M}\right|_{2}=|\mathfrak{M} \cap \mathfrak{M}|_{2}$. Hence, $\tilde{\mathfrak{N}}_{2}$ is a $S_{2}$-subgroup of $\mathfrak{S}_{1} \cap \mathfrak{M}$. Hence, $\Re_{0}$ is a maximal element of $\mathcal{U}_{\mathfrak{n}}\left(\mathfrak{R}_{p} ; 2\right)$. In particular, $\Re_{0} \supseteqq O_{2}(\mathfrak{M})$. Let $\Re_{1}$ be any subgroup of $\Re$ with $\boldsymbol{O}_{2}(\mathfrak{M}) \subseteq \Re_{1} \subseteq \Re$. Since $\boldsymbol{C}\left(\boldsymbol{O}_{2}(\mathfrak{M})\right)=\boldsymbol{Z}\left(\boldsymbol{O}_{2}(\mathfrak{M})\right)$, it follows that $Z\left(\Re_{1}\right) \subseteq Z\left(O_{2}(\mathfrak{M})\right)$.

Let $\mathfrak{M}_{0}=\boldsymbol{O}_{p^{\prime}}(\mathfrak{M})$. Since $\Re_{0}$ is a maximal element of $\boldsymbol{U}_{\mathfrak{M}}\left(\mathfrak{N}_{p} ; 2\right)$, it follows that $\mathfrak{M}_{0} \cap \mathfrak{R}_{0}$ is a $S_{2}$-subgroup of $\mathfrak{M}_{0}$. Let $\mathfrak{B}_{0}=\Omega_{1}\left(\boldsymbol{R}_{2}\left(\mathfrak{M}_{0}\right)\right)$, so that $\mathfrak{B}_{0}$ is 2-reducible in $\mathfrak{M}_{0}$. Let $\mathfrak{M}_{1}=C_{\mathfrak{n}}\left(\mathfrak{B}_{0}\right)$. We will show that $\mathfrak{M}_{1} \subseteq \mathfrak{M}_{0}$. Suppose false. Let $\Re_{1}=\Re \cap \mathfrak{M}_{0}$ so that $\mathfrak{R}_{1} \supseteqq \boldsymbol{O}_{2}(\mathfrak{M})$ and $\Re_{1}$ is a $S_{2}$-subgroup of $\mathfrak{M}_{0}$. By Lemma 5.10, $\mathfrak{B}_{0} \supseteqq \Omega_{1}\left(\boldsymbol{Z}\left(\Re_{1}\right)\right)$. In particular, $\mathfrak{B}_{0} \supseteqq \Omega_{1}(\mathbb{Z}(\mathfrak{R}))$. Since $\mathfrak{M}_{1} \triangleleft \mathfrak{M}$, and $\mathfrak{M}_{1} \nsubseteq \mathfrak{M}_{0}$, it follows that $p|| \mathfrak{M}_{1} \mid$. Hence, $p\left|\mathfrak{M}_{1} \cap C\left(\Omega_{1}(\boldsymbol{Z}(\Re))\right)\right|$. Let $\mathfrak{R}$ be a $S_{2, p}$-subgroup of $N\left(\Omega_{1}(Z(\Re))\right)$ which contains $\mathfrak{R}$. Thus, $\Re \nsubseteq \mathfrak{M}$, so by Lemma 13.23 , $S_{p}$-subgroups of $\Re$ are cyclic. Let $\mathfrak{P}_{0}$ be a subgroup of $\mathfrak{M}_{1} \cap C\left(\Omega_{1}(Z(\Omega))\right)$ of order $p$, so that $\Re_{0} \leqq N\left(\Omega_{1}(Z(\Re))\right)$. We can choose $B$ in $N\left(\Omega_{1}(Z(\Re))\right.$ ), so that $\mathfrak{N}_{p}=\mathfrak{P}_{0}^{B}$. Hence, $\mathfrak{N}_{p} \subseteq \mathfrak{M} \cap \mathfrak{M}^{B}$. Since $N\left(\mathfrak{N}_{p}\right) \subseteq \mathfrak{M}$, we get that one of the following holds:

(i) $S_{p}$-subgroups of $\mathfrak{M} \cap \mathfrak{M}^{B}$ are non cyclic.

(ii) $S_{p}$-subgroups of $\mathfrak{M}$ are cyclic.

If (i) holds, Lemma 13.23 implies that $\mathfrak{M}=\mathfrak{M}^{B}$, so that $B \in \mathfrak{M}$. If (ii) holds, then $p \in \tau$. But then Lemma 13.21 implies that $B \in \mathfrak{M}$. 
Hence, $B \in \mathfrak{M}$, so that $\mathfrak{R}_{p} \subseteq \mathfrak{M}_{1}$. Since $S_{p}$-subgroups of $\mathfrak{M}_{1}$ are cyclic, it follows that $\tilde{\mathfrak{N}}_{2}$ is contained in a $S_{2}$-subgroup $\mathfrak{T}^{*}$ of $\mathfrak{M}$ which is permutable with $\mathfrak{R}_{p}$. Since $\Re_{0}$ is a maximal element of $\boldsymbol{U}_{\mathfrak{m}}\left(\Re_{p} ; 2\right)$, it follows that $\Re_{0} \supseteqq \boldsymbol{O}_{2}\left(\mathfrak{T} * \mathfrak{R}_{p}\right)$. Hence, $\Re_{0}=\boldsymbol{O}_{2}\left(\mathfrak{T} * \mathfrak{R}_{p}\right)$, since $\boldsymbol{O}_{2}\left(\mathfrak{T} * \mathfrak{N}_{p}\right)$ is a maximal element of $\boldsymbol{U}_{\mathfrak{z}^{*} \Re_{p}}\left(\mathfrak{N}_{p} ; 2\right)$. Hence, $\mathfrak{T}^{*} \mathfrak{N}_{p} \subseteq N\left(\Re_{0}\right)$. Since $\subseteq \leqq N\left(\Re_{0}\right)$, we can choose $C$ in $N\left(\Re_{0}\right) \cap N\left(\Re_{p}\right)$ such that $\mathfrak{S}^{C} \supseteqq \mathfrak{T}^{*}$. Since $C \in N\left(\mathfrak{N}_{p}\right) \subseteq$ $\mathfrak{M}$, we get that $\mathfrak{S}$ contains a $S_{2}$-subgroup of $\mathfrak{M}$. This is not the case since $\subseteq \nsubseteq \mathfrak{M}$. We conclude that $\mathfrak{M}_{1} \subseteq \mathfrak{M}_{0}$. Hence, $\boldsymbol{O}_{2}\left(\mathfrak{M} \bmod \mathfrak{M}_{1}\right) \subseteq$ $\mathfrak{M}_{0}$, since $\mathfrak{M}_{0}=\boldsymbol{O}_{p^{\prime}}(\mathfrak{M})$ and $\boldsymbol{O}_{2}\left(\mathfrak{M} \bmod \mathfrak{M}_{1}\right)$ is a normal $p^{\prime}$-subgroup of $\mathfrak{M}$. Since $\mathfrak{B}_{0}$ is 2 -reducible in $\mathfrak{M}_{0}$, this implies that $\boldsymbol{O}_{2}\left(\mathfrak{M} \bmod \mathfrak{M}_{1}\right)=$ $\mathfrak{M}_{1}$, so that $\mathfrak{B}_{0}$ is 2-reducible in $\mathfrak{M}$. Hence, $\mathfrak{B}_{0} \subseteq \mathfrak{B}$. On the other hand, $\mathfrak{B}_{0} \supseteqq \Omega_{1}(\boldsymbol{Z}(\Re))$, since $\Re_{0} \cap \mathfrak{M}_{1}$ is a $S_{2}$-subgroup of $\mathfrak{M}_{1}$. Since $\mathfrak{B}^{\sharp} \subseteq \mathscr{J}$, we get $\Re \subseteq \mathfrak{M}$. This contradiction shows that this case does not arise.

\section{Case 2. $\mathfrak{R}$ is not a $2, p$-group.}

Let $\tilde{\mathfrak{N}}$ be a $S_{2, p}$-subgroup of $\mathfrak{R}$ which contains $\mathbb{2}$. Let $\mathfrak{R}_{2}, \mathfrak{R}_{p}$ be a Sylow system for $\tilde{\mathfrak{R}}$ with $P \in \mathfrak{N}_{p}$. Choose $q \in \pi(\mathfrak{N}), q \neq 2, p$, and let $\mathfrak{D}$ be a $S_{q}$-subgroup of $\mathfrak{N}$ which is permutable with $\mathfrak{R}_{2}$ and with $\mathfrak{R}_{p}$ such that $\mathfrak{M} \mathbb{M}$. Such a choice of $q$ is possible since $\mathfrak{R} \nsubseteq \mathfrak{M}$. By minimality of $|\mathfrak{N}|$, we have $\mathfrak{N}=\mathfrak{R}_{2} \mathfrak{N} \mathfrak{N}$.

Suppose $G \in \mathbb{S}$ and $\mathfrak{N} \subseteq \mathfrak{M}^{G}$. Then $P \in \mathfrak{M}^{G}$. Since $P \in \hat{\mathfrak{M}}_{p}$, it follows that $\mathfrak{M}=\mathfrak{M}^{G}$, against $\mathfrak{R} \nsubseteq \mathfrak{M}$. We conclude that $\mathfrak{R}$ is contained in no conjugate of $\mathfrak{M}$.

We next show that $\mathfrak{R}_{p}=\langle P\rangle$. This is clear if $\mathfrak{R}_{p}$ is cyclic, by minimality of $|\mathfrak{N}|$. Suppose by way of contradiction that $\mathfrak{R}_{p}$ is non cyclic. By Lemma 13.21, we get $p \notin \tau$, so by construction, we have $p \in \sigma$. Since $\mathfrak{R}_{p} \notin \mathscr{A}(p)$, it follows that $\mathfrak{R}_{p}$ contains a cyclic subgroup of index $p$. Hence, the $p$-length of $\mathfrak{R}$ is 1 . By minimality of $|\mathfrak{R}|$, we get $\mathfrak{N}_{p}=\Omega_{1}\left(\mathfrak{N}_{p}\right)$, since $P \in \Omega_{1}\left(\mathfrak{N}_{p}\right)$. Since $\mathfrak{N}_{p} \notin \mathscr{A}(p)$, it follows that $\mathfrak{R}_{p}$ contains an element $P_{1}$ such that $C_{\mathfrak{n}}\left(P_{1}\right)$ contains no elementary subgroup of order $p^{3}$. Hence, $\mathfrak{R}_{p}=\langle P\rangle \times\left\langle P_{1}\right\rangle$, since $P \in \hat{\mathfrak{M}}_{p}$. This implies that $N_{\mathfrak{m}}\left(\mathfrak{N}_{p}\right)$ permutes transitively the subgroups of $\mathfrak{N}_{p}$ of order $p$ distinct from $\langle P\rangle$. Hence, $\langle P\rangle \triangleleft N_{\mathfrak{m}}\left(\mathfrak{R}_{p}\right)$. But $N_{\mathfrak{m}}\left(\mathfrak{R}_{p}\right)$ contains an element of $\mathscr{A}(p)$, and so $N\left(\mathfrak{R}_{p}\right) \subseteq \mathfrak{M}$. Since $\mathfrak{R}=\boldsymbol{O}_{p^{\prime}}(\mathfrak{R}) \cdot \boldsymbol{N}_{\mathfrak{N}}\left(\mathfrak{R}_{p}\right)$, it follows that $\mathfrak{N}_{2}\langle P\rangle \Omega$ is a group, so by minimality of $|\mathfrak{N}|$, we get $\mathfrak{R}=\mathfrak{R}_{2}\langle P\rangle \mathfrak{l}$. This contradiction shows that $\mathfrak{R}_{p}=\langle P\rangle$.

Since $N\left(\mathfrak{R}_{p}\right) \subseteq \mathfrak{M}$, and since $\mathfrak{N} \nsubseteq \mathfrak{M}$, it follows that $\mathfrak{R}_{p} \nless \mathfrak{R}$. Hence, $\boldsymbol{O}_{p}(\mathfrak{N})=1$, since $|\mathfrak{N}|_{p}=p$.

Suppose by way of contradiction that $\boldsymbol{O}_{2}(\mathfrak{R})=1$. Since $\boldsymbol{O}_{p}(\mathfrak{R})=1$, it follows that $\boldsymbol{F}(\mathfrak{R})=\boldsymbol{O}_{q}(\mathfrak{R})$. Thus, $\mathfrak{X}$ is faithfully represented on $\boldsymbol{O}_{q}(\mathfrak{R})$, so by Lemma 5.11, $\mathscr{S} \mathscr{C} \mathscr{N}_{3}\left(\boldsymbol{O}_{q}(\mathfrak{R})\right) \neq \varnothing$. Hence, $q \in \pi_{3} \cup \pi_{4}$. Also, $2 \sim q$, since $\mathfrak{X}$ is a non cyclic abelian group of order 8 . Hence, 
$(q) \leqq \mathbb{L}^{*}(\mathbb{S})$, either by virtue of $q \in \pi_{3}$, or by virtue of $q \geqq 5, q \varepsilon \pi_{4}$. Here we are once again invoking Theorems 8.1 and 9.1 to conclude that if $q=3$, then $q \in \pi_{3}$. Let $\mathfrak{M}^{*}=M(\mathfrak{N})$. Thus, $\mathfrak{M}^{*} \neq \mathfrak{M}$. By maximality of $|\mathfrak{M} \cap \mathfrak{N}|_{2}$, it follows that $\mathfrak{R}_{2}$ is a $S_{2}$-subgroup of $\mathfrak{M}^{*}$. Let $\mathfrak{M}_{q}^{*}$ be a $S_{q}$-subgroup of $\mathfrak{M}^{*}$ permutable with $\mathfrak{N}_{2}$. By Lemma 6.6, either $\boldsymbol{O}_{2}\left(\mathfrak{M}_{q}^{*} \mathfrak{R}_{2}\right)=1$ or $\boldsymbol{O}_{q}\left(\mathfrak{R}_{2} \mathfrak{M}_{q}^{*}\right)=1$. Suppose $\boldsymbol{O}_{2}\left(\mathfrak{M}_{q}^{*} \mathfrak{R}_{2}\right)=1$. Since $\mathfrak{X} \subseteq \mathfrak{R}_{2}$, it follows that for some $X$ in $\mathfrak{X}^{*}, \boldsymbol{O}_{q}\left(\mathfrak{M}_{q}^{*} \mathfrak{R}_{2}\right) \cap C(X)$ contains an element of $\mathscr{A}(q)$. Hence, $C(X) \subseteq \mathfrak{M}^{*}$. By Lemma 13.1, $\boldsymbol{O}_{2^{\prime}}(\boldsymbol{C}(X))=1$. This is impossible since $\boldsymbol{O}_{q}\left(\mathfrak{M}_{q}^{*} \mathfrak{N}_{2}\right) \cap \boldsymbol{C}(X) \subseteq \boldsymbol{O}_{2^{\prime}}(\boldsymbol{C}(X))$. Hence, $\boldsymbol{O}_{2}\left(\mathfrak{M}_{q}^{*} \mathfrak{N}_{2}\right) \neq 1$. Hence, $N\left(\boldsymbol{O}_{2}\left(\mathfrak{M}_{q}^{*} \mathfrak{R}_{2}\right)\right) \subseteq \mathfrak{M}^{*}$, so that $\mathfrak{M}^{*}$ contains an element $\mathfrak{U}^{*}$ of $\mathscr{C}(2)$. Hence, $\boldsymbol{O}_{2}\left(\mathfrak{M}^{*}\right) \neq 1$, by Lemma 6.1. Hence, $\mathfrak{M}^{*}=N\left(\boldsymbol{O}_{2}\left(\mathfrak{M}^{*}\right)\right)$, since $\mathfrak{M}^{*} \in \mathscr{C} \mathscr{S}(\mathbb{S})$. By Lemma 13.1, we get $\boldsymbol{O}_{2^{\prime}}\left(\mathfrak{M}^{*}\right)=1$. Hence, $\boldsymbol{U}_{\mathfrak{w}^{*}}\left(\mathfrak{R}_{2} ; 2^{\prime}\right)$ is trivial. This violates $\boldsymbol{O}_{q}(\mathfrak{R}) \in$ $\boldsymbol{U}_{\mathfrak{M}} *\left(\mathfrak{R}_{2} ; 2^{\prime}\right)$. We conclude that $\boldsymbol{O}_{2}(\mathfrak{R}) \neq 1$. By maximality of $|\mathfrak{N} \cap \mathfrak{M}|_{2}$, together with the fact that Case 1 does not hold, $\Re_{2}$ is a $S_{2}$-subgroup of $\boldsymbol{N}\left(\boldsymbol{O}_{2}(\mathfrak{R})\right)$. This implies that $\boldsymbol{O}_{2^{\prime}}(\mathfrak{R})=1$, by Lemma 13.1. By Lemma 13.20, we get $\mathscr{S C C}_{\mathfrak{S}}(\mathfrak{\Omega})=\varnothing$.

Let $\mathfrak{I}^{*}$ be a $S_{2}$-subgroup of $\mathfrak{M}$ which contains $\mathfrak{R}_{2}$. By maximality of $\left|\mathfrak{R}_{2}\right|$, it follows that $\boldsymbol{Z}\left(\mathfrak{T}^{*}\right) \subseteq \mathfrak{R}_{2}$, and so $Z\left(\mathfrak{T}^{*}\right) \subseteq Z\left(\boldsymbol{O}_{2}(\mathfrak{R})\right)$. Hence,

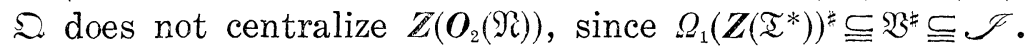

By minimality of $|\mathfrak{N}|, \mathfrak{N} \cap \mathfrak{M}$ is a maximal subgroup of $\mathfrak{N}$. Since $\left|\mathfrak{N}_{p}\right|=p$, and $\boldsymbol{N}\left(\mathfrak{N}_{p}\right) \subseteq \mathfrak{M}$, it follows that $\boldsymbol{O}_{2}(\mathfrak{N}) \cong \triangleleft \mathfrak{N}$. Hence, $\mathfrak{M} \cap \mathfrak{N}=$ $\mathfrak{R}_{2} \mathfrak{N}_{p} \boldsymbol{D}(\mathfrak{Q})$, and $\boldsymbol{O}_{2}(\mathfrak{M}) \mathfrak{O} / \boldsymbol{O}_{2}(\mathfrak{R}) \boldsymbol{D}(\mathfrak{\Omega})$ is a chief factor of $\mathfrak{R}$. Since $\mathscr{S}_{\mathscr{6}} \mathscr{N}_{3}(\mathfrak{D})=\varnothing,(0.3 .4)$ implies that either $\mathfrak{Q}$ is abelian or is a non abelian group of order $q^{3}$ and exponent $q$. Since $\left[\mathfrak{D}, \mathfrak{N}_{p}\right] \neq 1$, we get $q \equiv 1(\bmod p)$, so $q \geqq 5$.

Let $\mathfrak{W}$ be a minimal normal 2-subgroup of $\mathfrak{R}$ which is not centralized by $\mathfrak{Q} ; \mathfrak{W}$ exists since $1 \neq\left[\boldsymbol{R}_{2}(\mathfrak{R}), \mathfrak{N}\right] \triangleleft \mathfrak{R}$ 。 Let $\mathfrak{R}_{0}=\boldsymbol{C}_{\mathfrak{n}}(\mathfrak{W})$. Thus, $\Re_{0} \subseteq \mathfrak{R}_{2} \mathfrak{N}_{p} \boldsymbol{D}(\mathfrak{\Omega})$, and so $\mathfrak{R}_{0} \subseteq \mathfrak{R}_{2} \boldsymbol{D}(\mathfrak{Q})$, since $\mathfrak{Q}=\left[\mathfrak{\Omega}, \mathfrak{N}_{p}\right]$. Since $q \geqq 5$, Theorem 1 of [43] implies that $\mathfrak{R}_{2} \cong=C_{\mathfrak{R}_{2} \mathrm{a}}\left(Z\left(\mathfrak{R}_{2}\right)\right)$. $\boldsymbol{N}_{\mathfrak{\Re}_{2} \Omega}\left(J\left(\mathfrak{N}_{2}\right)\right)$. Since $\boldsymbol{C}\left(\boldsymbol{Z}\left(\mathfrak{R}_{2}\right)\right) \subseteq \mathfrak{M}$, and since $\mathfrak{M} \cap \mathfrak{Q}=\boldsymbol{D}(\mathfrak{D})$, it follows that $\mathfrak{Q}$ normalizes $\boldsymbol{J}\left(\mathfrak{R}_{2}\right)$, so that $J\left(\Re_{2}\right) \subseteq \boldsymbol{O}_{q^{\prime}}(\mathfrak{R})=\boldsymbol{O}_{2}(\mathfrak{R})$. It follows that $\boldsymbol{J}\left(\mathfrak{N}_{2}\right) \triangleleft \mathfrak{N}$, so by maximality of $|\mathfrak{R} \cap \mathfrak{M}|_{2}$, we conclude that $\mathfrak{R}_{2}$ is a $S_{2}$-subgroup of $\mathfrak{M}$. Clearly, $\mathfrak{W} \cap \mathbb{Z}\left(\mathfrak{R}_{2}\right) \neq 1$. Since $\mathfrak{W} \cap \mathbb{Z}\left(\mathfrak{R}_{2}\right) \subseteq \mathfrak{B}$, and since $\boldsymbol{D}(\mathfrak{\Omega}) \subseteq \mathfrak{M}$, it follows that $D(\mathfrak{Q})$ centralizes $\mathfrak{W} \cap Z\left(\mathfrak{N}_{2}\right)$. Since $\mathfrak{W}$ is a minimal normal subgroup of $\mathfrak{N}$, we get $[D(\mathfrak{D}), \mathfrak{W}]=1$. Let $\overline{\mathfrak{N}}=\mathfrak{N} / \mathfrak{N}_{0}$ and for any subset $\mathfrak{Y}$ of $\mathfrak{R}$, let $\overline{\mathfrak{Y}}$ be the image of $\mathfrak{Y}$ in $\overline{\mathfrak{R}}$. Thus, $\overline{\mathfrak{D}}$ is a normal abelian subgroup of $\overline{\mathfrak{N}}$ and $\left[\overline{\mathfrak{N}}, \overline{\mathfrak{N}}_{p}\right] \neq 1$. Let $\mathfrak{W}_{0}=\mathfrak{W} \cap \boldsymbol{Z}\left(\mathfrak{R}_{2}\right)$. Since $\mathfrak{R}_{2}$ is a $S_{2}$-subgroup of $\mathfrak{M}$, we have $\mathfrak{W}_{0} \leqq \mathfrak{B}$, by Lemma 5.10 . Thus, $\left|\mathfrak{W}_{0}\right| \leqq 4$, by Lemma 13.19. By Lemma 5.46, we get $\left|\mathfrak{N}_{0}\right|=4$, whence $\mathfrak{S}_{0}=\mathfrak{N}$. But $\mathfrak{S} \triangleleft \mathfrak{M}$, against Lemma 5.46. This contradiction completes the proof of this lemma.

Let $\mathscr{F}_{0}$ be the set of all the normal elementary 2-subgroups of $\mathfrak{M}$ of order $\geqq 8$. Thus, $\mathscr{F}_{0}$ is partially ordered by inclusion. Let 
$\mathscr{F}$ be the set of all minimal elements of $\mathscr{F}_{0}$.

Hypothesis 13.2.

(a) Hypothesis 13.1 is satisfied.

(b) $\mathscr{F}_{0} \neq \varnothing$.

Let $\mathfrak{F}$ be an element of $\mathscr{F}$. Choose $\mathfrak{F}$ such that $\mathfrak{F} / \mathbb{F}$ is a chief factor of $\mathfrak{M}$. Let $\mathfrak{M}_{0}=\boldsymbol{C}_{\mathfrak{M}}(\mathfrak{F}), \mathfrak{E}=\boldsymbol{C}_{\mathfrak{M}}(\mathfrak{F}), \mathfrak{D}=\boldsymbol{O}_{2}\left(\mathfrak{M}_{0} \bmod \mathfrak{E}\right)$. This notation is preserved in Lemmas 13.25 through 13.38 .

LEMMA 13.25 .

(a) $|\mathfrak{F}| \leqq 4$

(b) $\quad$ s $\neq 1$.

Proof. Since $\mathfrak{F} \in \mathscr{F}$, (a) holds. If $\mathfrak{F}=1$, then $\mathfrak{F}$ is 2 -reducible in $\mathfrak{M}$, against Lemma 13.19 and Lemma 5.9 (i).

LEMma 13.26.

(a) If $I$ is an involution of $\mathfrak{M}$ and $C_{\mathfrak{n}}(I) \cap \hat{\mathfrak{M}}_{p} \neq \varnothing$ for some $p \in \sigma \cup \tau$, then $I \in \mathscr{J}$.

(b) $\quad \xi^{\#} \subseteq \mathscr{I}$.

(c) One of the following holds:

(i) $\mathfrak{F}^{\prime} \subseteq \mathscr{J}$.

(ii) $\mathfrak{F}$ is generated by subgroups $\mathfrak{X}$ of order 16 such that $\mathfrak{U} \cong \mathscr{\mathcal { F }}$.

Proof. Since $2 \in \pi_{4}$, it follows that $C_{\mathfrak{n}}(I)$ contains an elementary subgroup of order 8. Thus, (a) is a consequence of Lemma 13.24. Since $|\mathfrak{F}| \leqq 4$, (b) is a consequence of (a). If $|\mathfrak{F}|=8$, then (a) yields (c)(i), so suppose $|\mathfrak{F}| \geqq 16$.

Choose $p \in \sigma$ and let $\mathfrak{B}$ be an elementary subgroup of $\mathfrak{M}$ of order $p^{3}$. Let $\mathfrak{F}=\mathfrak{F}_{0} \times \widetilde{F}_{1} \times \cdots \times \widetilde{F}_{s}$, where each $\mathfrak{\mho}_{i}$ is an irreducible $\mathfrak{B}$ group, $1 \leqq i \leqq s$, and $\mathfrak{F}_{0}=C_{\mathfrak{\mho}}(\mathfrak{B})$. Let $\mathfrak{B}_{i}=C_{\mathfrak{B}}\left(\mathfrak{\mho}_{i}\right), \mathfrak{B}_{i j}=\mathfrak{B}_{i} \cap \mathfrak{B}_{j}$. Thus, $\mathfrak{B}_{i j} \neq 1$. By Lemma 13.24, it follows that $\left(\mathfrak{\mho}_{0} \mathfrak{\mho}_{i} \mathfrak{\mho}_{j}\right)^{\#} \subseteq \mathscr{J}$. If $s \leqq 1$, then $\mathfrak{B}$ is not faithful on $\mathfrak{F}$, and so $\mathfrak{F} \cong \mathscr{J}$, so that (c)(ii) follows. If $s \geqq 2$, then $\left|\widetilde{\mho}_{0} \widetilde{\mho}_{i} \widetilde{\mho}_{j}\right| \geqq 16$ for $1 \leqq i<j \leqq s$. Since $\widetilde{\mho}_{0} \widetilde{\mho}_{i} \widetilde{\mho}_{j}$ is generated by its subgroups of order 16, (c)(ii) holds and the proof is complete.

Lemma 13.27. Suppose $\mathfrak{F}_{0}$ is a subgroup of $\mathfrak{F}$ of index 2 and $\mathfrak{A}$ is an elementary 2-subgroup of $\mathfrak{M}$ such that

(a) $\mathfrak{A} \cap \boldsymbol{C}(\mathfrak{F})=1$.

(b) $C_{\mathfrak{F}}(\mathfrak{Z})=\widetilde{F}_{0}$.

Then $|\mathfrak{N}: \mathfrak{A} \cap \mathfrak{D}| \leqq 2$.

Proof. First, suppose that $\mathfrak{A}$ does not centralize (5. Choose $E \in \mathfrak{F}-C(\mathfrak{Q})$. By (b), we get $\mathfrak{\Im}=\mathfrak{F}_{0} \times\langle E\rangle$. By (a), no element of 
$\mathfrak{X}^{*}$ centralizes $\mathfrak{5}$. Thus, $\mathfrak{X}$ is faithfully represented as automorphisms of $\mathfrak{5}$, a four-group. Hence, $|\mathfrak{U}| \leqq 2$, and we are done. We may assume that $\mathfrak{F} \subseteq \widetilde{F}_{0}$, so that $\mathfrak{U} \subseteq \mathfrak{M}_{0}$.

Let $\mathfrak{U}_{0}$ be a complement for $\mathfrak{U} \cap \mathfrak{D}$ in $\mathfrak{A}$, and let $\left|\mathfrak{A}_{0}\right|=2^{a}$. We must show that $a \leqq 1$. Since $\mathfrak{N}_{0} \cap \mathfrak{D}=1$, no element of $\mathfrak{N}_{0}$ stabilizes the chain $\mathscr{C}: \mathfrak{F} \supset \mathbb{F} \supset 1$. Since $\mathfrak{R}_{0}$ centralizes $\mathfrak{F}$, it follows that no element of $\mathfrak{U}_{0}^{*}$ centralizes $\mathfrak{W}=\mathfrak{F} / \mathfrak{F}$. Let $\mathfrak{W}_{0}=\mathfrak{F}_{0} / \mathfrak{F}$, so that $\mathfrak{W}_{0}$ is a hyperplane of $\mathfrak{W}$, and $\mathfrak{S}_{0}=C_{\mathfrak{2 B}}(A)$ for all $A$ in $\mathfrak{U}_{0}^{\ddagger}$. Since $\mathfrak{M}_{0} / \boldsymbol{C}_{\mathfrak{\mathfrak { R } _ { 0 }}}(\mathfrak{W})$ has no normal 2 -subgroup and is solvable, we conclude that $\left|\mathfrak{R}_{0}\right| \leqq 2$.

LEMma 13.28. One of the following holds:

(a) $\mathfrak{F} \cong \mathscr{J}$.

(b) $C_{\Re}(\widetilde{f})$ is a $(\sigma \cup \tau)^{\prime}$-group.

Proof. Suppose (b) does not hold. If $\boldsymbol{C}_{\mathfrak{m}}(\mathfrak{F})$ is not a $\tau^{\prime}$-group, then (a) holds by Lemma 13.24, together with the fact that for each $p$ in $\tau, \hat{\mathfrak{M}}_{p}$ contains every element of $\mathfrak{M}$ of order $p$. Suppose $p \in \sigma$, and $p \| C_{\mathfrak{n}}(\widetilde{F}) \mid$. Let $\mathfrak{P}$ be a $S_{p}$-subgroup of $C(\mathfrak{F})$, and let $\mathfrak{P}^{*}$ be a $S_{p}$-subgroup of $\mathfrak{M}$ which contains $\mathfrak{P}_{\text {. Then }} 1 \subset \mathfrak{P}=\mathfrak{P}^{*} \cap \boldsymbol{C}(\mathfrak{F}) \triangleleft \mathfrak{P}^{*}$. Since $\mathfrak{P} \triangleleft \mathfrak{P}^{*}, \mathfrak{P}$ contains an element of $\hat{\mathfrak{M}}_{p}$, so (a) holds by Lemma 13.24. The proof is complete.

The next lemma is the heart of the matter.

Lemma 13.29. Suppose $J$ is an involution of $\mathfrak{M}-C_{9 R}(\mathfrak{F})$. Let $\widetilde{\vartheta}_{0}=C_{\widetilde{\mho}}(J), 2^{f}=\left|\widetilde{F}: \widetilde{F}_{0}\right| \cdot$

(a) If $\left[\mathfrak{F} \nsubseteq \widetilde{F}_{0}\right.$ then $[\widetilde{\mho}, J] \cap \mathscr{J} \neq \varnothing$.

(b) Suppose $\left(5 \subseteq \widetilde{F}_{0}\right.$.

(i) If $f \leqq 2$, then $[\widetilde{F}, J]^{\ddagger} \subseteq \mathscr{J}$.

(ii) If $f=3$ and $\widetilde{F}_{1}$ is a subgroup of $\mathfrak{F}$ of index 2 which contains $\widetilde{\mho}_{0}$, then $\left[\widetilde{\mho}_{1}, J\right] \cap \mathscr{S} \neq \varnothing$.

Proof. By Lemma 13.28, we may assume that $C(\hat{\wp})$ is a $(\sigma \cup \tau)^{\prime}-$ group, since the conclusions of this lemma are obvious if $\hat{F}^{*} \subseteq \mathscr{F}$.

If $\left[\mathfrak{F} \nsubseteq \widetilde{F}_{0}\right.$, then we get $[\widetilde{F}, J] \supseteqq[\widetilde{F}, J] \supset 1$. Thus, (a) holds, by Lemma 13.26(b). For the remainder of the proof, we assume that

$$
\mathfrak{F} \subseteq \widetilde{\mho}_{0}, \quad J \in \mathfrak{M}_{0} \text {. }
$$

For each subset $\subseteq$ of $\mathfrak{M}_{0}$, let $\bar{\Xi}=\subseteq \mathfrak{D} / \mathfrak{D}$. Let $\Re$ be the set of

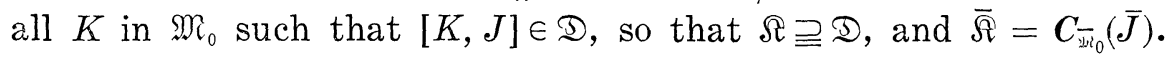

Since $\widetilde{S} / \mathfrak{G}$ is a chief factor of $\mathfrak{M}$ which is centralized by $\mathfrak{D}$, we

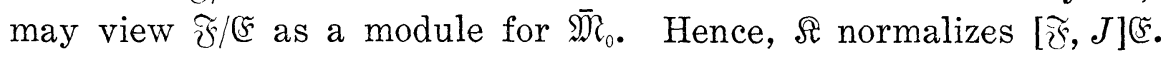

Suppose $\mathfrak{X}$ is a subgroup of $\Omega$ such that for each $p$ in $\sigma \cup \tau$, every element of $\mathfrak{X}$ of order $p$ is in $\hat{\mathfrak{M}}_{p}$ and such that $|\mathfrak{X}|_{\sigma\lrcorner=} \neq 1,3,7$. Since $\Re$ normalizes $[\widetilde{F}, J] \mathfrak{F}$, so does $\mathfrak{X}$. Since 


$$
[\mathfrak{F}, J] \mathfrak{s} /[\mathfrak{s} \cong[\mathfrak{F}, J] /[\mathfrak{F}, J] \cap \mathfrak{E},
$$

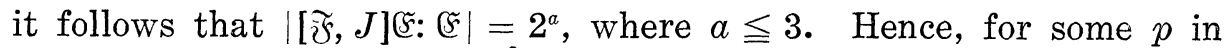
$\sigma \cup \tau$, some element of $\mathfrak{X} \cap \hat{\mathfrak{N}}_{p}$ centralizes [₹r, $\left.J\right] \mathfrak{F}$, and we are done. We may assume that no such subgroups $\mathfrak{X}$ exist.

Case 1. $\mathfrak{M}_{0}$ contains a subgroup $\mathfrak{R}$ such that

$(\alpha) \quad \Re \supset \mathfrak{D},|\Re: \mathfrak{D}|=p^{r}, p \in \sigma \cup \tau$.

( $\beta$ ) $J$ normalizes $\Re$.

( $\gamma$ ) Every element of $\mathfrak{R}$ of order $p$ is in $\hat{\mathfrak{M}}_{p}$.

$(\delta)$ One of the following holds:

(i) $p=3, r=3, \Re / \mathcal{D}$ is elementary.

(ii) $p=3, r \geqq 4$.

(iii) $p=7, r \geqq 3$.

(iv) $p=7, \Re$ contains an element $R$ of order 7 which is inverted by $J$ and such that for some prime $q$ in $\sigma-\{7\}, C_{\mathfrak{R}_{0}}(R)$ contains an element of $\mathscr{A}(q)$.

$$
\text { (v) } p \neq 3,7, p^{r}>5 \text {. }
$$

Let $\tilde{\Re}=\langle\Re, J\rangle$ and let $\mathfrak{S}, \mathfrak{S}_{1}, \mathfrak{S}_{0}, X$ be the images in $A_{\mathfrak{\Re}_{0}}(\mathfrak{\Im})$ of $\tilde{\Re}, \Re$, $D$ D, $J$ respectively. If $(\delta)(\mathrm{i})$ or $(\delta)$ (ii) holds, then we may apply Lemma 5.48 or Lemma 5.49 with $\widetilde{\xi}$ in the role of the $F_{2} \subseteq$-module $M$. Translating the conclusions of these lemmas to the present situation, and then using $(\gamma)$ and Lemma 13.24 yields this lemma. Suppose $(\delta)$ (iii) holds. If $J$ inverts a subgroup of $\Re / \mathbb{C}$ of order $7^{2}$, then there is a subgroup $\mathfrak{P}$ of $\mathfrak{R} / \boldsymbol{C}(\mathfrak{F}) \cap \Re$ of order $7^{2}$ which is inverted by $J$, by Lemma

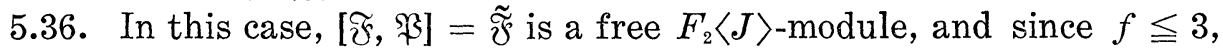
we get $|\tilde{\mho}|=2^{6}$. Also, $\mathfrak{P}$ is represented faithfully on $\tilde{\mho}$. Thus, $\langle J, \mathfrak{P}\rangle$ is isomorphic to a subgroup of $\operatorname{GL}(2,6)$. This is impossible since GL $(6,2)$ has $S_{7}$-subgroups of order $7^{2}$ and also has elements of order 7 which are not real.

Let $\Re_{0} / \mathfrak{D}=\boldsymbol{C}_{\Re / /}(J)$. Thus, $\Re_{0}$ normalizes $[\mathfrak{F}, J] \mathfrak{F}$. Since $\Re_{0}$ cen-

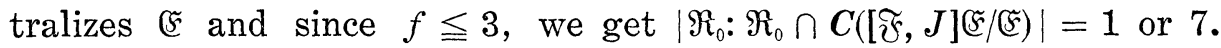
If $\left|\Re_{0}: \mathfrak{D}\right|>7$, then $\Re_{0}$ contains an element of order 7 which centralizes $[\mathfrak{F}, J]$ and we are done. On the other hand, $\Re_{0} \supset \mathfrak{D}$, since $J$ does not invert $\mathfrak{R} / \mathfrak{D}$. Hence, $\left|\Re_{0}: \mathfrak{D}\right|=7$. Since $J$ inverts no subgroup of $\Re / \mathfrak{D}$ of order $7^{2}$, and since $|\Re: \mathfrak{D}| \geqq 7^{3}$, it follows that $\Re / \mathfrak{D}$ is a non abelian group of order $7^{3}$ and exponent 7 , and that $J$ inverts the Frattini quotient group of $\Re / \mathfrak{D}$. This is impossible, since $f \leqq 3$.

Suppose $(\delta)$ (iv) holds. Let $\tilde{\mho}=[\widetilde{\mho}, R]$. Thus, $|\tilde{\mho}|=2^{6}, f=3$. Let $\mathfrak{A}$ be an element of $\mathscr{A}(q)$ which centralizes $\Re$. Then $\tilde{\mathfrak{F}}$ admits $\mathfrak{A}$, and since $q \neq 2,7$, it follows that $\mathfrak{A}$ centralizes $\tilde{\mho}$. But $\tilde{\mho} \supseteqq[\widetilde{F}, J]$, so we are done.

Suppose $(\delta)(\mathrm{v})$ holds. Again, let $\Re_{0} / \mathfrak{D}=C_{\Re / \mathbb{D}}(J)$. Then $\mathfrak{R}_{0}$ normalizes $[\mathfrak{F}, J] \mathfrak{F}$, so a $S_{p}$-subgroup of $\Re_{0}$ centralizes $[\mathfrak{F}, J] \mathfrak{F}$, since 
$\mid[\tilde{\xi}, J] \mathfrak{s}:\left\{\mathfrak{F} \mid \leqq 2^{3}\right.$ and $p \neq 3,7$. If $\Re_{0} \supset \mathfrak{D}$, we are done, so suppose $J$ inverts $\Re / \mathcal{D}$. Since $p^{r}>5$, we get $f \geqq 4$. Thus, if any of the above possibilities occur, we are done.

Let $\mathbb{R} / \mathbb{D}$ be the Fitting subgroup of $\mathfrak{M}_{0} / \mathfrak{D}$. Thus, $\mathbb{R} / \mathbb{D}$ is of odd order. Let $\mathfrak{\Omega}_{1}$ be a $S_{\sigma^{\prime} \mathrm{s}}$-subgroups of $\mathbb{2}$, so that $\mathfrak{\Omega}_{1} \cong \bar{\Omega}_{1}$. Let $p$ be the largest prime in $\sigma$, and let $\mathfrak{P}$ be a $S_{p}$-subgroup of $\mathfrak{M}$. Since $\mathfrak{P} \subseteq \mathfrak{M}^{\prime}, \mathfrak{P}$ centralizes every cyclic factor of $\mathfrak{M}$.

\section{Case $2 . \quad p \geqq 5$.}

Since neither $(\delta)(\mathrm{i})$ nor $(\delta)$ (ii) hold, either the $S_{3}$-subgroup of $\bar{\Omega}_{1}$ contains no elementary subgroup of order $3^{3}$ or $3 \notin \sigma$. If $3 \notin \sigma$, then $\mathfrak{F}$ centralizes every chief 3 -factor of $\mathfrak{M}$, since $\mathfrak{M}$ has no elementary subgroup of order $3^{3}$. If $3 \in \sigma$, then we still get that $\mathfrak{P}$ centralizes the $S_{3}$-subgroup of $\mathbb{R}_{1}$, since $\left|\mathfrak{Q}_{1}\right|_{3}<3^{4}$, and $\mathfrak{R}_{1}$ contains no elementary subgroup of order $3^{3}$.

Suppose $q$ is a prime such that $\mathfrak{P}$ does not centralize the $S_{q^{-}}$ subgroup of $\mathbb{2}$. We will show that $q=p$. If $q \neq p$, we get $q \neq 3$, and by Lemma 13.22, we have $q \in \sigma \cup \tau$. Since $p$ is the largest prime in $\sigma$, and $p \geqq 5$, it follows that one of ( $\delta$ )(iii), (iv), (v) is satisfied for a suitable subgroup $\Re / \mathfrak{D}$ of $\bar{\Omega}$. We may assume that $\mathfrak{P}$ centralizes the $S_{p^{\prime}}$-subgroup of $\bar{\Omega}$. Hence, $\mathfrak{P}$ centralizes $\boldsymbol{O}_{p^{\prime}}\left(\mathfrak{M}_{0} / \mathfrak{D}\right)$, from which we conclude that $\mathbb{R} / \mathcal{D}$ contains an elementary subgroup of order $p^{3}$. It follows that one of ( $\delta$ )(iii), (iv), (v) holds for a suitable $p$-subgroup of $\mathrm{R}$.

\section{Case 3. $p=3$.}

In this case, we get $\sigma=\{3\}$. If $\mathfrak{P}$ does not centralize the $S_{3^{\prime}}$ subgroup of $\bar{\Omega}$, then one of $(\delta)($ iv), (v) holds and we are done. We may assume that $\mathfrak{P}$ centralizes the $S_{3}$-subgroup of $\bar{\Omega}$.

Let $\mathfrak{B}_{0}=\mathfrak{P} \cap \mathbb{R}$, so that $\overline{\mathfrak{P}}_{0}$ is the $S_{3}$-subgroup of $\bar{\Omega}$. First, suppose that $\mathfrak{P}_{0}$ contains an element $P$ of order 3 which is not in $\hat{\mathfrak{M}}_{3}$. Choose $\mathfrak{U} \in \mathscr{Z}(\mathfrak{P})$ and let $\mathfrak{P}_{1}=C_{\mathfrak{P}_{0}}(\mathfrak{l})$. Thus, $\mathfrak{P}_{0}=\mathfrak{P}_{1}\langle P\rangle$. Since $(\delta)(\mathrm{i})$, (ii) fail, it follows that $\left|\mathfrak{P}_{1}\right| \leqq 3^{3}$ and $\mathfrak{P}_{1}$ contains no elementary subgroup of order $3^{3}$. Hence, $\mathfrak{M}=\Omega_{1}\left(\mathfrak{P}_{1}\right)$. Suppose in addition that $\mathfrak{P}_{0}$ contains a non cyclic characteristic abelian subgroup. In this case, we get $\mathfrak{U}$ char $\mathfrak{P}_{0}$, so that $\overline{\mathfrak{U}}$ is a factor of $\mathfrak{M}$. But then $\left[\overline{\mathfrak{U}, \mathfrak{P}_{0}}\right]$ is a chief factor of $\mathfrak{M}$ so that $\mathfrak{B D}$ centralizes every chief 3 -factor of $\mathfrak{M}$ which is between $\mathfrak{D}$ and $\mathcal{L}$. This forces $\mathfrak{P} \cap \mathfrak{M}_{0}=\mathfrak{P}_{0}$, and also gives $\mathfrak{P D} \triangleleft \mathfrak{M}$. Since $J$ normalizes $\mathfrak{P},(\delta)(i)$ is satisfied for a suitable subgroup $\Re$ of $\mathfrak{P D}$. We may assume that every characteristic abelian subgroup of $\mathfrak{P}_{0}$ is cyclic, and that $\mathfrak{D P} \rtimes \mathfrak{M}$. This forces $\Omega_{1}\left(\mathfrak{P}_{0}\right)$ to be non abelian of order $3^{3}$. Since $\mathfrak{P}$ does not centralize $\Omega_{1}\left(\mathfrak{P}_{0}\right) / \boldsymbol{D}\left(\Omega_{1}\left(\mathfrak{P}_{0}\right)\right)$, owing to $\mathfrak{P D} \measuredangle \mathfrak{M}$, 
we get that all elements of $\mathfrak{P}_{0}$ of order 3 are in $\hat{\mathfrak{M}}_{3}$.

It remains now to consider the case where every element of $\mathfrak{B}_{0}$ of order 3 is in $\hat{\mathfrak{M}}_{3}$. As $(\delta)(\mathrm{i}),(\delta)$ (ii) do not hold, $\mathfrak{P}_{0}$ is of order at most $3^{3}$ and if $\left|\mathfrak{P}_{0}\right|=3^{3}, \mathfrak{P}_{0}$ is not an elementary. Since $\mathfrak{D P} \pitchfork \mathfrak{M}$, it follows that $\mathfrak{B}_{0}$ is non abelian of order $3^{3}$ and $\mathfrak{B} \cong Z_{3} \int Z_{3}$.

We next show that $\overline{\mathfrak{L}}=\overline{\mathfrak{B}}_{0}$. Suppose false. Let $r$ be the largest prime divisor of $|\overline{\mathfrak{L}}|$, so that $r \geqq 5$. Let $\Re$ be a $S_{r}$-subgroup of $\mathfrak{M}$. Then $\Re$ centralizes the $S_{r^{\prime}}$-subgroup of $\mathbb{L}$, and so $\Re \subseteq \mathbb{R}$. By definition of $\tau$, together with $\sigma=\{3\}$, we get $r \in \tau$. Suppose $r \neq 7$. Then $|\mathfrak{R}|=5$, since otherwise $(\delta)(\mathrm{v})$ holds. If $J$ inverts $\bar{\Re}$, then $\mathfrak{M}$ has an element $R$ of order 5 inverted by $J$, and $[\mathfrak{F}, R]=\tilde{F}$ is of order $2^{4}$. But $\tilde{\widetilde{F}} \triangleleft \mathfrak{M}$, against the presence of $\mathfrak{P}_{0}$, together with the fact that $\widetilde{F} / \mathfrak{F}$ is a chief factor of $\mathfrak{M}$. If $J$ centralizes $\bar{\Re}$, then $\Re$ normalizes $[\widetilde{\mho}, J] \mathfrak{F}$, so that a $S_{5}$-subgroup of $\mathfrak{M}$ centralizes $[\mathfrak{F}, J]$. This is impossible, since $\hat{\mathfrak{M}}_{5}$ contains every element of $\mathfrak{M}$ of order 5 .

Suppose $r=7$. If $J$ inverts some element of $\Re D$ of order 7 , we are done, since $(\delta$ )(iv) holds. We may assume that $J$ centralizes $\bar{\Re}$. Hence, $\Re \mathfrak{D}$ normalizes $[\mathfrak{F}, J] \mathfrak{F}$, so we may assume that $|\Re|=7$. Hence, $\overline{\mathfrak{L}}$ is the direct product of $\overline{\mathfrak{P}}_{0}$ and $\bar{\Re}$. Now $\mathfrak{F} / \mathbb{F}$ is a chief factor of $\mathfrak{M}$, so $\mathfrak{P}_{0}^{\prime}$ and $\mathfrak{R}$ have no non trivial fixed points on $\mathfrak{F} / \mathbb{F}$. Since $J$ does not centralize $\mathfrak{F}_{0}$, it follows that $|\mathfrak{F}: \mathfrak{F}|=2^{6}$, owing to $f \leqq 3$. But no element of GL $(6,2)$ of order 7 centralizes any extra special subgroup of order $3^{3}$. Hence, $\bar{\Omega}=\overline{\mathfrak{P}}_{0}$, from which we get that $\overline{\mathfrak{M}}$ is a 2, 3-group.

We now get $\mathfrak{D} \mathfrak{R}_{0} \triangleleft \mathfrak{M}$. Let $\mathfrak{M}_{1}=\mathfrak{M}_{0} \mathfrak{P}$. Since $\mathfrak{M} / \mathfrak{M}_{0}$ is 3 -closed, we get $\mathfrak{M}_{1} \triangleleft \mathfrak{M},\left|\mathfrak{M}: \mathfrak{M}_{1}\right| \leqq 2$. Also, $\boldsymbol{O}_{2}\left(\mathfrak{M}_{1} / \mathfrak{D}\right)=1$, and the 3-length of $\mathfrak{M}_{1} / \mathscr{D}$ is 2. If $J \in O_{3,2}\left(\mathfrak{M}_{1} \bmod \mathfrak{D}\right)$, then since $S_{2}$-subgroups of $\boldsymbol{O}_{3,2}\left(\mathfrak{M}_{1} \bmod \mathfrak{D}\right) / \mathbb{R}$ are quaternion, it follows that $J$ normalizes $\mathfrak{P} \mathfrak{D}$. This is impossible since $(\sigma)$ (ii) does not hold. Hence, we may assume that $J \notin \boldsymbol{O}_{3,2}\left(\mathfrak{M}_{1} \bmod (\mathfrak{D})\right.$. In this case, $S_{2}$-subgroups of $\mathfrak{M}_{1} / \mathbb{R}$ are isomorphic to $S_{2}$-subgroups of GL $(2,3)$. Every involution of GL $(2,3)$ normalizes some $S_{3}$-subgroup of GL $(2,3)$, and so $J$ normalizes some $S_{3}$-subgroup of $\mathfrak{M}_{1} / \mathcal{D}$, against the fact that $(\delta)$ (ii) does not hold. The proof is complete.

Lemma 13.30. Suppose $G \in \mathbb{S}$ and $\mathfrak{\mho}^{G} \subseteq \mathfrak{M}$. Then $\mathfrak{F}^{G} \subseteq \mathfrak{C}$.

Proof. Let $\mathfrak{F}^{*}=\mathfrak{F}^{G}, \mathfrak{M}^{*}=\mathfrak{M}^{G}, \mathfrak{F}^{*}=\mathfrak{F}^{G}, \mathfrak{D}^{*}=\mathfrak{D}^{a}, \mathfrak{C}^{*}=\mathfrak{C}^{G}, \mathscr{J}^{*}=$ $\mathscr{J}^{G}$. Since $\mathfrak{F}$ is abelian, we may assume that $G \notin \mathfrak{M}$, so that $\mathfrak{M} \neq \mathfrak{M}^{*}$. Hence, $\mathscr{J} \cap \mathscr{J}^{*}=\varnothing$.

First, suppose $\mathfrak{F}^{\#} \subseteq \mathscr{J}$. Then $\mathfrak{F}^{* *} \subseteq \mathscr{J}^{*}$. Let $\mathfrak{F}_{0}=C_{\mathfrak{F}}\left(\mathfrak{F}^{*}\right)$, and suppose by way of contradiction that $\widetilde{F}_{0} \subset \mathfrak{F}_{\text {. }}$ Let $\widetilde{F}_{1} / \widetilde{\mho}_{0}$ be a chief factor of $\mathfrak{F}^{*}$ with $\widetilde{F}_{1} \subseteq \mathfrak{F}_{\text {. Choose }} F \in \widetilde{F}_{1}-\widetilde{F}_{0}$. Since $\left|\mathfrak{F}^{*}\right|=\left|\mathfrak{F}^{*}\right|$, we get $\left|\mathfrak{F}_{0}\right|<\left|\mathfrak{F}^{*}\right|$. Hence, there is $F^{*}$ in $\mathfrak{F}^{*}-\{1\}$ such that $\left[F, F^{*}\right]=1$. 
Since $F^{*} \in \mathscr{J}^{*}$, we get $F \in \mathfrak{M}^{*}$. Hence, $1 \neq\left[F, \mathfrak{\Im}^{*}\right] \subseteq \mathfrak{F} \cap \mathfrak{F}^{*}$, against $\mathscr{F} \cap \mathscr{J}^{*}=\varnothing$.

We may now assume that $\xi^{\#} \nsubseteq \mathscr{F}$. By Lemma 13.26 (c), it follows that if $\widetilde{F}_{1}^{*}$ is any subgroup of $\Im^{*}$ of index at most 8 , then $\boldsymbol{C}\left(\mathfrak{F}_{1}^{*}\right) \subseteq \mathfrak{M}^{*}$. We will show that $\mathfrak{F}^{*}$ centralizes $\mathfrak{F}$. Let $\mathfrak{F}_{1}^{*}=\boldsymbol{C}_{\mathfrak{\gamma}^{*}}(\mathfrak{F})$, so that $\left|\mathfrak{F}^{*}: \mathfrak{F}_{1}^{*}\right| \leqq 2$. Suppose $\left|\mathfrak{F}^{*}: \mathfrak{F}_{1}^{*}\right|=2$. Choose $E$ in $\mathfrak{F}-\boldsymbol{C}\left(\mathfrak{F}^{*}\right)$. Thus, $E \in \mathfrak{M}^{*}$. We may apply Lemma 13.29 (c), with $E$ in the role of $J, \mathfrak{M}^{*}$ in the role of $\mathfrak{M}, \widetilde{\mho}_{1}^{*}$ in the role of $\widetilde{F}_{0}$. We get $\left[\mathfrak{\mho}^{*}, E\right]^{*} \subseteq \mathscr{F}^{*}$. But $\left[\mathfrak{F}^{*}, E\right]^{*} \cong \mathscr{J}$, against $\mathscr{J} \cap \mathscr{I}^{*}=\varnothing$. Hence, $\mathfrak{F}^{*}$ centralizes $\mathfrak{F}^{*}$.

Suppose $\mathfrak{F}^{*} \subseteq \mathfrak{D}$, but $\mathfrak{F}^{*} \nsubseteq \mathfrak{F}$. Choose $F$ in $\mathfrak{F}-\boldsymbol{C}\left(\mathfrak{F}^{*}\right)$, and let $\mathfrak{F}_{2}^{*}=C_{\mathscr{F}^{*}}(F)$. Then $\left|\mathfrak{F}^{*}: \mathfrak{F}_{2}^{*}\right| \leqq 4$, since $\left[F, \mathfrak{F}^{*}\right] \leqq \mathfrak{F}^{*}$. Hence, $F \in \mathbb{M}^{*}$. Hence, $\left[\varsigma^{*}, F\right] \subseteq \Im \cap \varsigma^{*}=1$, so $\varsigma^{*} \subseteq \widetilde{\mho}_{2}^{*}$. By Lemma 13.29 (b)(i), we get $\left[\mathfrak{F}^{*}, F\right]^{\ddagger} \leqq \mathscr{I}^{*}$, against $1 \subset\left[\mathfrak{\mho}^{*}, F\right] \leqq \mathfrak{F}$.

Thus, in proving this lemma we may assume that $\mathfrak{\Im}^{*} \subseteq \mathfrak{M}_{0}, \mathfrak{F}^{*} \nsubseteq \mathfrak{D}$. Let $\mathfrak{P} / \mathfrak{D}$ be a subgroup of $\mathfrak{M}_{0} / \mathfrak{D}$ of odd prime order $p$ which admits $\mathfrak{F}^{*}$ and is not centralized by $\mathfrak{F}^{*}$. We will exploit $\mathfrak{P} / \mathcal{D}$ to show that $\widetilde{F} \subseteq \mathfrak{M}^{*}$, introducing a powerful symmetry.

Namely, let $\widetilde{\mho}_{3}^{*}=C_{\mathscr{\mho}^{*}}(\mathfrak{P} / \mathfrak{D})$, so that $\left|\mathfrak{F}^{*}: \widetilde{\mathfrak{F}}_{3}^{*}\right|=2$. Let $\overline{\widetilde{\mho}}=\widetilde{F} / \mathfrak{F}$, and $\overline{\mathscr{F}}_{1}=\widetilde{F}_{1} / \mathfrak{S}$ be a subgroup of $\widetilde{F}$ which admits $\left\langle\mathfrak{F}, \widetilde{F}^{*}\right\rangle$ and is minimal subject to $\left[\overline{\mathscr{F}}_{1}, \mathfrak{F}\right] \neq 1$. Then $\widetilde{F}_{3}^{*} \mathfrak{D}$ centralizes $\overrightarrow{\mathscr{V}}_{1}$ and $\widetilde{F}^{*}$ does not. Choose $F$ in $\widetilde{F}_{1}$ such that $\widetilde{F}^{*}$ does not centralize $\mathfrak{s} F$. Let $\widetilde{F}_{4}^{*}=\widetilde{F}_{3}^{*} \cap$ $C(F)$. Since $\left[\widetilde{F}_{1}, \mathfrak{F}_{3}^{*}\right] \leqq\left[\mathfrak{F}_{\text {, we get }}\left|\mathfrak{F}_{3}^{*}: \mathfrak{F}_{4}^{*}\right|=2^{a}\right.$, with $a \leqq 2$. Hence, $\left|\mathfrak{F}^{*}: \widetilde{F}_{4}^{*}\right| \leqq 8$, so that $C\left(\widehat{\mho}_{4}^{*}\right) \subseteq \mathfrak{M}^{*}$. Hence, $F \in \mathfrak{M}^{*}$.

Since $F \in \mathfrak{M}^{*}$, we get $\left[F, \mathfrak{F}^{*}\right] \subseteq \widetilde{F} \cap \mathfrak{F}^{*}$. If $\mathfrak{F} \cap \mathfrak{F}^{*} \neq 1$, then $\mathfrak{F} \subseteq$ $C\left(\Im_{\curlyvee} \cap \mathfrak{F}^{*}\right) \subseteq \mathfrak{M}^{*}$, as we are trying to show. So suppose $\widetilde{F}^{*} \cap \mathfrak{F}^{*}=1$. In this case $\mathfrak{F}^{*} \subseteq \mathfrak{V}_{4}^{*}$.

If $a=2$, we apply Lemma 13.29 (b)(ii) with $F$ in the role of $J$, $\mathfrak{M}^{*}$ in the role of $\mathfrak{M}, \widetilde{F}_{4}^{*}$ in the role of $\widetilde{F}_{0}$, $\widetilde{F}_{3}^{*}$ in the role of $\widetilde{F}_{1}$. We get $\left[\mathfrak{F}_{3}^{*}, F\right] \cap \mathscr{F}^{*} \neq \varnothing$, against $1 \subset\left[\mathfrak{F}_{3}^{*}, F\right] \leqq[$. If $a \leqq 1$, we apply Lemma $13.29(\mathrm{~b})(\mathrm{i})$ and get $\left[\mathfrak{F}^{*}, F\right]^{\sharp} \subseteq \mathcal{F}^{*}$. Thus, $\mathscr{J}^{*} \cap \mathfrak{F} \neq \varnothing$ in both cases, so that $\widetilde{\wp} \cong \mathfrak{M}^{*}$.

We now define the integer $b$ by $2^{b}=\left|\mathfrak{F}^{*}: \mathfrak{F}^{*} \cap \mathfrak{D}\right|$. As we have seen, $b \geqq 1$. Let $\overline{\mathfrak{M}}_{0}=\mathfrak{M}_{0} / \mathfrak{D}$. Thus, $\overline{\mathscr{F}}$ is a $F_{2} \overline{\mathfrak{M}}_{0}$-module. Since $\mathfrak{F} \subseteq \mathfrak{M}^{*}$, we get $[\mathfrak{F}, A, B]=1$ for all $A, B \in \mathfrak{F}^{*}$. Let $\mathfrak{X} / \mathfrak{D}$ be a subgroup of $\overline{\mathfrak{M}}_{0}$ which admits $\mathfrak{F}^{*} \mathfrak{D} / \mathfrak{D}$, such that $\mathfrak{X} / \mathfrak{D}=\mathfrak{X}_{1} / \mathfrak{D} \times \cdots \times \mathfrak{X}_{l} / \mathfrak{D}$, where each $\mathfrak{X}_{i}$ admits $\mathfrak{F}^{*}$ and $C_{\mathfrak{F}^{*}}(\mathfrak{X} / \mathfrak{D})=\mathfrak{F}^{*} \cap \mathfrak{D}$, and where $\left|\mathfrak{X}_{i}: \mathfrak{D}\right|=p_{i}$, a prime. We can then choose elements $F_{1}^{*}, \cdots, F_{b}^{*}$ of $\mathfrak{F}^{*}$, such that $F_{i}^{*}$ inverts $\mathfrak{X}_{i} / \mathfrak{D}$ and centralizes $\mathfrak{X}_{j} / \mathfrak{D}$ for $j \neq i$. Thus, $\mathfrak{F}^{*}=\mathfrak{F}^{*} \cap \mathfrak{D} \times$ $\left\langle F_{1}^{*}\right\rangle \times \cdots \times\left\langle F_{b}^{*}\right\rangle$. Also, since $F_{i}^{*}$ inverts $\mathfrak{X}_{i} / \mathscr{D}, \mathfrak{X}_{i}$ contains an element $X_{i}$ of odd order which is inverted by $F_{i}^{*}$ and satisfies $\mathfrak{X}_{i}=\left\langle\mathfrak{D}, X_{i}\right\rangle$. Let $\overline{\mathscr{\mho}}_{1}=\widetilde{F}_{1} / \mathfrak{F}$ be an irreducible subgroup of $\overline{\mathfrak{\mho}}$ as $F_{2}\left\langle\mathfrak{X}, \mathfrak{F}^{*}\right\rangle$-module such that $\mathfrak{X}$ does not centralize $\overline{\mathscr{\mho}}_{1} ; \overline{\mathscr{\mho}}_{1}$ exists since $\mathfrak{X} / \mathcal{D}$ acts faithfully on $\overline{\mathscr{F}}$ and $b \geqq 1$, and since $\overline{\mathscr{F}}$ is a completely reducible $\mathfrak{X} / \mathfrak{D}$-group.

Suppose $\mathfrak{X}_{i} / \mathfrak{D}$ centralizes $\overline{\mathscr{V}}_{1}$ for some $i$. Then $X_{i}$ stabilizes $\widetilde{F}_{1} \supset$ 
(Fつ1, so since $X_{i}$ has odd order, $X_{i}$ centralizes $\widetilde{F}_{1}$. On the other hand, we can choose $j$ such that $\mathfrak{X}_{j} / \mathfrak{D}$ does not centralize $\overline{\mathscr{F}}_{1}$. Thus, letting $\mathfrak{X}_{j} / \mathfrak{D}$ play the role of $\Re / \mathcal{D}$ in the first part of the lemma, it follows that $\widetilde{F}_{1} \cap \mathscr{J}^{*} \neq \varnothing$. Thus, $X_{i} \in \mathfrak{M}^{*}$. This is absurd, since $F_{i}^{*}$ inverts $X_{i}$, while $\mathfrak{F}^{*} \triangleleft \mathfrak{M}^{*}$. We conclude that $\mathfrak{X}_{i} / \mathfrak{D}$ has no non trivial fixed points on $\overline{\mathscr{F}}_{1}, 1 \leqq i \leqq b$.

By Lemma 5.47 with $\left\langle\mathfrak{X}, \mathfrak{F}^{*}, \mathfrak{D}\right\rangle / \mathfrak{D}$ in the role of $\mathfrak{S}, \overline{\mathscr{F}}_{1}$ in the role of $\mathfrak{M}$ and with $k=F_{2}$, we conclude that $b=1$.

By symmetry, we get $\left|\mathfrak{F}: \mathfrak{F} \cap \mathfrak{D}^{*}\right|=2$. Also, $\left[\mathfrak{F} \cap \mathfrak{D}^{*}, \mathfrak{F}^{*} \cap \mathfrak{D}\right] \subseteq$ $\mathfrak{F} \cap \mathfrak{F}^{*}=1$, so $\left\langle\mathfrak{F} \cap \mathfrak{D}^{*}, \mathfrak{F}^{*} \cap \mathfrak{D}\right\rangle$ is abelian.

We next show that $\mathfrak{F}^{*} \cong \mathfrak{F}^{*} \cap \mathfrak{D}$. Suppose false and $E^{*} \in \mathfrak{F}^{*}-$ $\mathfrak{F}^{*} \cap \mathfrak{D}$. Since $\mathfrak{F} \subseteq \mathfrak{M}^{*}$, we get $\left|\mathfrak{F}: C_{\mathfrak{F}}\left(E^{*}\right)\right|=2$. By Lemma 13.29(a) with $E^{*}$ in the role of $J$, we get $\left[\widetilde{F}, E^{*}\right]^{\sharp} \subseteq \mathscr{J}$. This is impossible, since $\left[\mathfrak{F}, E^{*}\right] \subseteq \mathfrak{F}^{*}$. Hence, $\mathfrak{F}^{*} \subseteq \mathfrak{F}^{*} \cap \mathfrak{D}$. By symmetry, $\mathfrak{F} \subseteq \mathfrak{D}^{*} \cap \mathfrak{F}$.

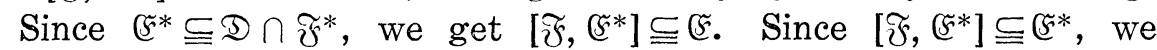
conclude that $\mathfrak{F}^{*}$ centralizes $\mathfrak{F}$. By symmetry, $\mathfrak{F}$ centralizes $\mathfrak{F}^{*}$.

Choose $F \in \widetilde{F}-\widetilde{F} \cap \mathfrak{D}^{*}$. Then $F$ centralizes $\mathfrak{F}^{*}$. Let $\tilde{\mho}^{*}=\mathfrak{F}^{*} \cap$ $C(F)$. Since $\left[\mathfrak{F}^{*} \cap \mathfrak{D}, F\right] \subseteq\left(\mathfrak{F}\right.$, it follows that $\left|\mathfrak{F}^{*}: \tilde{\mho}^{*}\right| \leqq 8$. Suppose $\left|\mathfrak{F}^{*}: \tilde{\mathscr{F}}^{*}\right|=8$. By Lemma 13.29 (b)(ii) with $F$ in the role of $J, \mathfrak{M}^{*}$ in the role of $\mathfrak{M}, \widetilde{\mho}^{*}$ in the role of $\widetilde{F}_{0}, \mathfrak{F}^{*} \cap \mathfrak{D}$ in the role of $\widetilde{F}_{1}$, we get $\left[\mathfrak{F}^{*} \cap \mathfrak{D}, F\right] \cap \mathscr{J}^{*} \neq \varnothing$. This is impossible, since $\left[\mathfrak{F}^{*} \cap \mathfrak{D}, F\right] \subseteq(\mathfrak{F}$. If $\left|\mathfrak{F}^{*}: \widetilde{\mathfrak{V}}^{*}\right|=4$, then Lemma $13.29(\mathrm{~b})(\mathrm{i})$ yields a contradiction, since $\left[\mathfrak{F}^{*} \cap \mathfrak{D}, F\right] \neq 1$. Hence, we may assume that $\mathfrak{F}^{*} \cap \mathfrak{D} \subseteq \mathbb{C}$, since otherwise, $\left[\mathfrak{F}^{*}, F\right]^{*} \subseteq \mathscr{I}^{*}$, against $1 \subset\left[\mathfrak{F}^{*} \cap \mathfrak{D}, F\right] \subseteq \mathfrak{F}$.

Now $F$ centralizes a hyperplane of $\mathfrak{F}^{*}$. Choose $F^{*} \in \mathfrak{F}^{*}-\mathfrak{F}^{*} \cap \mathfrak{D}$. Then by symmetry $F^{*}$ centralizes a hyperplane of $\mathfrak{F}$. Hence, [ $\left.\mathfrak{F}, \mathfrak{F}^{*}\right]=$ $\left[F, \mathfrak{F}^{*}\right]=\left[\mathfrak{F}, F^{*}\right]=\left\langle\left[F, F^{*}\right]\right\rangle$ is of order 2 , so by two applications of Lemma $13.29(\mathrm{~b})(\mathrm{i})$, we get $\left[F, F^{*}\right] \in \mathscr{J} \cap \mathscr{J}^{*}$. This contradiction completes the proof.

LEMma 13.31. One of the following holds:

(a) $\mathfrak{F} \cong \mathscr{I}$.

(b) $\boldsymbol{C}\left(\widetilde{\mho}_{0}\right) \subseteq \mathfrak{M}$ for every subgroup $\widetilde{\mho}_{0}$ of index 16 in $\widetilde{\mho}_{\text {. }}$

Proof. Suppose (a) fails. Choose $p \in \sigma$, and let $\mathfrak{P}$ be an elementary subgroup of $\mathfrak{M}$ of order $p^{3}$. Thus, $\mathfrak{F}$ is represented faithfully on $\mathfrak{F}$, by Lemma 13.28. Let $\widetilde{F}=\mathfrak{X}_{0} \times \mathfrak{X}_{1} \times \cdots \times \mathfrak{X}_{s}$, where $\mathfrak{X}_{0}=\boldsymbol{C}_{\mathfrak{F}}(\mathfrak{P})$ and $\mathfrak{X}_{1}, \cdots, \mathfrak{X}_{s}$ are irreducible $\mathfrak{P}$-groups. Thus, $\left(\mathfrak{X}_{0} \mathfrak{X}_{i} \mathfrak{X}_{j}\right)^{\#} \subseteq \mathscr{F}$ for all $i, j$, so we may assume that $\mathfrak{X}_{0} \mathfrak{x}_{i} \mathfrak{x}_{j} \cap \mathfrak{F}_{0}=1$ for all $i, j, 1 \leqq i<j \leqq s$. Since $\left|\mathfrak{F}_{i} \mathfrak{F}_{j}\right| \geqq 16$ whenever $1 \leqq i<j \leqq s$, we get $p=3, \mathfrak{X}_{0}=1,\left|\mathfrak{X}_{i}\right|=$ $4,1 \leqq i \leqq s$. Since $\mathfrak{P}$ acts faithfully on $\mathfrak{F}, s \geqq 3$. Let $\mathfrak{U}=\mathfrak{F}_{0} \cap \mathfrak{X}_{1} \mathfrak{X}_{2} \mathfrak{X}_{3}$, so that $|\mathfrak{U}| \geqq 4$. If $\mathfrak{U} \cap \mathfrak{X}_{i} \mathfrak{X}_{j} \neq 1$, for some $i, j$, we are done. Hence, we may assume that $\mathfrak{A}=\left\langle U_{1}, U_{2}\right\rangle$, where $U_{i}=X_{i 1} X_{i 2} X_{i 3}, X_{i j} \in \mathfrak{X}_{j}, X_{i j} \neq 1$, $1 \leqq j \leqq 3, i=1,2$. Since $U_{1} U_{2} \notin \mathfrak{X}_{i} \mathfrak{X}_{j}$ for all $i, j$, we get $\mathfrak{X}_{j}=\left\langle U_{1 j}, U_{2 j}\right\rangle$. 
Now $\mathfrak{P}=\mathfrak{P}_{1} \times \mathfrak{P}_{2} \times \mathfrak{P}_{3}$, where $\left|\mathfrak{P}_{i}\right|=3$ and $\left[\mathfrak{P}_{i}, \mathfrak{X}_{j}\right]=1, i \neq j, 1 \leqq i, j \leqq 3$. We can therefore choose $P_{i}$ in $\mathfrak{P}_{i}$ such that $P_{i}^{-1} X_{1 i} P_{i}=X_{2 i}, 1 \leqq i \leqq 3$. Let $P=P_{1} P_{2} P_{3}$. Then $U_{1}^{P}=U_{2}, U_{2}^{P}=U_{1} U_{2}$, so that $P$ normalizes $\mathfrak{u}$. Hence, $\quad C\left(\widetilde{F}_{0}\right) \subseteq C(\mathfrak{U}) \subseteq N(\mathfrak{U}) \subseteq \mathfrak{M}$, the last containment holding by Lemma 13.24.

Lemma 13.32. One of the following holds:

(a) $\mathfrak{F}^{*} \subseteq \mathscr{J}$.

(b) If $G \in \mathbb{S}$ and $\left|\mathfrak{F}^{G}: \mathfrak{F}^{G} \cap \mathfrak{M}\right| \leqq 2$, then $\mathfrak{F}^{G} \leqq \mathfrak{M}$.

Proof. Suppose (a) and (b) fail. Choose $G$ in (s) such that $\left|\mathfrak{F}^{G}: \mathfrak{F}^{G} \cap \mathfrak{M}\right|=2$. Let $\mathfrak{M}^{*}=\mathfrak{M}^{G}, \mathfrak{F}^{*}=\mathfrak{F}^{G}$, and let $\mathfrak{F}_{1}^{*}=\mathfrak{F}^{*} \cap \mathfrak{M}$. By Lemma 13.30, we get $\mathfrak{F} \nsubseteq \mathfrak{M}^{*}$. Hence, $\mathscr{J}^{*} \cap \mathfrak{F}=\varnothing, \mathscr{J} \cap \mathfrak{F}^{*}=\varnothing$.

Let $\mathfrak{F}_{0}=\mathfrak{F}_{\mathfrak{W}} \cap \mathfrak{M}^{*}$ so that $\mathfrak{F}_{0} \subset \mathfrak{F}_{\text {. }}$ Choose $F \in \mathfrak{F}-\widetilde{F}_{0}$. Let $\mathfrak{F}_{2}^{*}=$ $C(F) \cap \widetilde{\mho}_{1}^{*}$ and let $2^{f}=\left|\mathfrak{F}_{1}^{*}: \mathfrak{F}_{2}^{*}\right|$. If $f \leqq 3$, then $\left|\mathfrak{F}^{*}: \mathfrak{F}_{2}^{*}\right| \leqq 16$, so that by Lemma 13.31, $C\left(\mathfrak{F}_{2}^{*}\right) \subseteq \mathfrak{M}^{*}$, against $F \notin \mathfrak{M}^{*}$. Hence, $f \geqq 4$. In particular, $\mathfrak{F}_{1}^{*} \nsubseteq \mathfrak{D}$, since $\widetilde{F}_{0} \subset \mathfrak{F}^{*}$ Also, we get $\mathfrak{F} \subseteq \mathfrak{M}^{*}$, so that $\left[\mathfrak{F}_{1}^{*}, E\right] \subseteq \mathfrak{F}^{*} \cap \mathfrak{F}=1$, the equality holding since $\mathscr{F} \cap \mathfrak{F}^{*}=\varnothing$.

Let $\Re / D$ be a subgroup of $\mathfrak{M}_{0} / \mathcal{D}$ of odd prime order which admits $\mathfrak{F}_{1}^{*}$ and is not centralized by $\mathfrak{F}_{1}^{*}$. Let $\widetilde{F}_{2}^{*}=\widetilde{F}_{1}^{*} \cap \boldsymbol{C}(\Re / \mathfrak{D})$ so that $\left|\mathfrak{F}_{1}^{*}: \widetilde{F}_{2}^{*}\right|=2$. Let $\widetilde{F}_{1} / \mathfrak{F}$ be a subgroup of $\mathfrak{F}_{/} / \mathfrak{F}$ which admits $\Re \widetilde{F}_{1}^{*}$ and

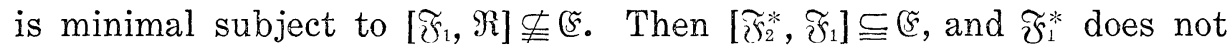

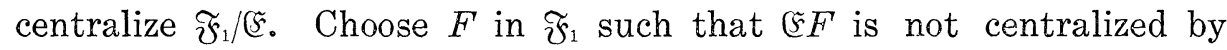
$\mathfrak{F}_{1}^{*}$. Since $\left[\mathfrak{F}_{2}^{*}, F\right] \subseteq \mathfrak{F}$, it follows that $\mathfrak{F}_{3}^{*}=\mathfrak{F}_{2}^{*} \cap C(F)$ is of index at most 4 in $\widetilde{F}_{2}^{*}$, so is of index at most 16 in $\mathfrak{F}^{*}$. Hence, $C\left(\mathfrak{F}_{3}^{*}\right) \subseteq \mathfrak{M}^{*}$, so that $F \in \mathfrak{M}^{*}$. Hence, $\left[\mathfrak{F}_{2}^{*}, F\right] \subseteq\left[\mathfrak{F} \cap \mathfrak{F}^{*}=1\right.$, so that $\mathfrak{F}_{3}^{*}=\mathfrak{F}_{2}^{*}$. Now $F \in \mathfrak{M}^{*}$ and $\mathfrak{F}^{*} \cap \boldsymbol{C}(F) \supseteqq \mathfrak{F}_{2}^{*}$, so that $\left|\mathfrak{F}^{*}: \mathfrak{F}^{*} \cap \boldsymbol{C}(F)\right| \leqq 4$. On the other hand, $\sqrt{5} \subseteq \mathfrak{M}^{*}$, so that $\mathfrak{F}^{*}=\mathfrak{F}^{*} \subseteq \mathfrak{M}$. Hence, $\mathfrak{F}^{*} \subseteq \mathfrak{F}_{1}^{*}$, so that $\left[\mathfrak{F}^{*}, F\right] \leqq \widetilde{F} \cap \mathfrak{F}^{*}=1$; that is, $\mathfrak{F}^{*} \subseteq \mathfrak{F}_{2}^{*}$.

By Lemma 13.29(b)(i) applied with $F$ in the role of $J, \mathfrak{M}^{*}$ in the role of $\mathfrak{M}, \mathfrak{F}_{2}^{*}$ in the role of $\mathfrak{F}_{0}, \mathfrak{F}^{*}$ in the role of $\mathfrak{F}$, we get $\left[\mathfrak{F}^{*}, F\right] \subseteq$ $\mathscr{J}^{*}$. In particular, $\left[\mathfrak{F}_{1}^{*}, F\right] \subseteq \mathscr{J}^{*}$. But $1 \subset\left[\mathfrak{F}_{1}^{*}, F\right] \subseteq \mathfrak{F}$, so we get $\mathscr{F}^{*} \cap \mathfrak{F} \neq \varnothing$. This contradiction completes the proof.

\section{LEMMA 13.33. $\mathfrak{F}^{*} \leqq \mathscr{F}$.}

Proof. Suppose false. By Lemma 13.28, $C(\mathfrak{F})$ is a $(\sigma \cup \tau)^{\prime}$-group. Let $\mathfrak{W}=V\left(\operatorname{ccl}_{\mathscr{S}}(\mathfrak{F}) ; \mathfrak{I}\right)$, where $\mathfrak{I}$ is a $S_{2}$-subgroup of $\mathfrak{M}$. By Lemma $13.30, \mathfrak{W} \subseteq C_{\mathfrak{m}}(\mathfrak{F})=\mathfrak{C}$, so $N_{\mathfrak{M}}(\mathfrak{B})$ contains a $S_{\sigma ! s}$-subgroup of $\mathfrak{M}$. Hence, $N(\mathfrak{B}) \subseteq \mathfrak{M}$, since $N_{\mathfrak{M}}(\mathfrak{B}) \in \mathscr{Z}^{*}(\mathbb{S})$.

Since $\mathfrak{F}^{*} \nsubseteq \mathscr{F}$, we can choose $F \in \mathfrak{F}^{\sharp}-\mathscr{F}$. Hence, $C_{\mathfrak{n}}(F) \notin \mathbb{C}^{*}(\mathbb{S})$. Let $\mathfrak{I}_{0}$ be a $S_{2}$-subgroup of $\boldsymbol{C}_{\mathfrak{n}}(F)$. Thus, $\mathfrak{I}_{0} \notin \mathscr{C l}^{*}(\mathbb{S})$. Let $\mathscr{T}=$ $\left\{\mathfrak{I}_{1} \mid \mathfrak{I}_{1}\right.$ is a 2 -subgroup of $\mathfrak{M}, \mathfrak{I}_{1} \supseteqq \mathfrak{I}_{0}^{H}$ for some $M$ in $\left.\mathfrak{M}, \mathfrak{I}_{1} \notin \mathbb{C l}^{*}(\mathbb{S})\right\}$. Thus, $\mathscr{T} \neq \varnothing$, as $\mathfrak{I}_{0} \in \mathscr{T}$. Let $\mathfrak{I}_{1}$ be a maximal element of $\mathscr{T}$. 
Since $\mathscr{T}=\mathscr{T}^{M}$ for all $M$ in $\mathfrak{M}$, we assume without loss of generality that $\mathfrak{I}_{1} \leqq \mathfrak{T}$. Since $\mathfrak{F} \subseteq \mathfrak{T}_{0}$, every element of $\mathscr{T}$ contains $\mathfrak{F}$.

Choose $\mathfrak{S} \in \mathscr{L} \mathscr{S}(\mathbb{S}), \mathfrak{I}_{1} \subseteq \mathfrak{S}, \mathfrak{S} \neq \mathfrak{M}$. Since $\mathfrak{F} \subseteq \mathfrak{S}$, we can choose a four-group $\tilde{\mho}$ of $\widetilde{F}$ with $\widetilde{\mho} \sharp \subseteq \mathscr{F}$. Hence,

$$
\boldsymbol{O}_{2^{\prime}}(\mathfrak{S})=\left\langle\boldsymbol{O}_{2^{\prime}}(\mathfrak{S}) \cap \boldsymbol{C}(\widetilde{F}) \mid \widetilde{F} \in \tilde{\mathfrak{F}^{\sharp}}\right\rangle,
$$

and so $\boldsymbol{O}_{2^{\prime}}(\mathfrak{S}) \subseteq \mathfrak{M}$. In particular, $\widetilde{F}$ centralizes $\boldsymbol{O}_{2^{\prime}}(\mathfrak{S})$, and so $\boldsymbol{O}_{2}(\mathfrak{S}) \neq 1$. Since $\mathfrak{S} \in \mathscr{C l} \mathscr{S}(\mathbb{S})$, we have $\mathfrak{S}=\boldsymbol{N}\left(\mathrm{O}_{2}(\mathfrak{S})\right)$, and so by Lemma 13.1, we have $\boldsymbol{O}_{2^{\prime}}(\mathfrak{S})=1$.

By maximality of $\mathfrak{I}_{1}$, it follows that $\mathfrak{I}_{1}$ is a $S_{2}$-subgroup of $\mathfrak{S}$. Let $\mathfrak{W}_{1}=V\left(\operatorname{ccl}_{\mathfrak{G}}(\mathfrak{\mho}) ; \mathfrak{T}_{1}\right)$. Since $\mathfrak{\mho} \subseteq \mathfrak{W}_{1}$, we have $\mathfrak{W}_{1} \neq 1$. We argue that $N\left(\mathfrak{W}_{1}\right) \subseteq \mathfrak{M}$. This is clear if $\mathfrak{I}_{1} \subset \mathfrak{I}$, since in this case, $N_{\mathfrak{\Sigma}}\left(\mathfrak{W}_{1}\right) \supset$ $\mathfrak{I}_{1}$, so that maximality of $\mathfrak{I}_{1}$ forces $N\left(\mathfrak{W}_{1}\right) \subseteq \mathfrak{M}$. If $\mathfrak{I}_{1}=\mathfrak{I}$, then $\mathfrak{W}_{1}=\mathfrak{W}$, and again we have $N\left(\mathfrak{W}_{1}\right) \subseteq \mathfrak{M}$, by the first paragraph of the proof.

Let $\cong$ be a subgroup of $\subseteq$ which is permutable with $\mathfrak{I}_{1}$, and is minimal subject to $\mathfrak{Q} \nsubseteq \mathfrak{M}$. Then $\mathfrak{Q}$ is a $q$-group for some odd prime $q$, and $\mathfrak{W}_{1} ₫ \mathfrak{D} \mathfrak{I}_{1}=\mathfrak{S}_{1}$, say. By minimality of $\mathfrak{Q}$, it follows that the $q$-length of $\mathfrak{S}_{1}$ is 1 , and that $\boldsymbol{O}_{2}\left(\mathfrak{S}_{1}\right) \mathfrak{\Omega}_{2} / \boldsymbol{O}_{2}\left(\mathfrak{S}_{1}\right) \boldsymbol{D}(\mathfrak{\Omega})$ is a chief factor of $\mathfrak{S}_{1}$.

Since $\mathfrak{W}_{1} \nsubseteq \boldsymbol{O}_{2}\left(\mathfrak{S}_{1}\right)$, we can choose $G$ in $\mathbb{S}$ such that $\mathfrak{F}^{G}=\mathfrak{F}^{*} \cong \mathfrak{T}_{1}$, $\mathfrak{F}^{*} \nsubseteq \boldsymbol{O}_{2}\left(\mathfrak{S}_{1}\right)$. Let $\mathfrak{Z}_{0}$ be a subgroup of $\mathfrak{\Omega}$ which is minimal subject to

(a) $\boldsymbol{O}_{2}\left(\mathfrak{S}_{1}\right) \mathfrak{\Omega}_{0}$ admits $\mathfrak{F}^{*}$,

( b ) $\left[\mathfrak{N}_{0}, \mathfrak{F}^{*}\right] \nsubseteq \mathfrak{M}$.

Since $\mathfrak{D}$ satisfies $(\mathrm{a})$ and $(\mathrm{b})$, owing to $\mathfrak{M} \mathfrak{M}=\boldsymbol{D}(\mathfrak{D}), \mathfrak{\Omega}_{0}$ exists. By minimality of $\mathfrak{\Omega}_{0}, \boldsymbol{O}_{2}\left(\mathfrak{S}_{1}\right) \mathfrak{\Omega}_{0} / \boldsymbol{O}_{2}\left(\mathfrak{S}_{1}\right)\left(\mathfrak{\Omega}_{0} \cap \boldsymbol{D}(\mathfrak{Q})\right)$ is an irreducible $\mathfrak{F}^{*}$-group. Since $\mathfrak{F}^{*}$ is an elementary, we get that $\left|\mathfrak{\Omega}_{0}: \mathfrak{\Omega}_{0} \cap \boldsymbol{D}\left(\Omega_{1}\right)\right|=q$, so that $\mathfrak{\Omega}_{0}$ is cyclic. Hence, if $\mathfrak{F}_{1}^{*}=\boldsymbol{C}_{\widetilde{\mho}^{*}}\left(\boldsymbol{O}_{2}\left(\mathfrak{S}_{1}\right) \mathfrak{\Omega}_{0} / \boldsymbol{O}_{2}\left(\mathfrak{S}_{1}\right)\right)$, then $\mathfrak{\mho}_{1}^{*}$ is of index 2 in $\mathfrak{F}^{*}$. Let $Q$ be a generator for $\mathfrak{\Omega}_{0}$. Since $\mathfrak{F}_{1}^{*} \boldsymbol{O}_{2}\left(\mathfrak{S}_{1}\right) \triangleleft \boldsymbol{O}_{2}\left(\mathfrak{S}_{1}\right) \mathfrak{Q}_{0} \mathfrak{F}^{*}$, we get $\mathfrak{F}_{1}^{* Q} \subseteq \mathfrak{M}$. Hence, $\left|\mathfrak{F}^{G Q}: \mathfrak{F}^{G Q} \cap \mathfrak{M}\right| \leqq 2$. By Lemma 13.32, we get $\mathfrak{F}^{G Q} \subseteq \mathfrak{M}$. Hence, $\left\langle\mathfrak{F}^{G}, \mathfrak{F}^{G Q}\right\rangle \cdot \boldsymbol{O}_{2}\left(\mathfrak{S}_{1}\right) \subseteq \mathfrak{M}$. But since

$$
\mathfrak{\Omega}_{0} \subseteq\left\langle\mathfrak{F}^{G}, \mathfrak{F}^{G Q}\right\rangle \boldsymbol{O}_{2}\left(\mathfrak{S}_{1}\right) \text {, }
$$

we have a contradiction, since $\mathfrak{\Omega}_{0} \nsubseteq \mathfrak{M}$. The proof is complete.

Again, let $\mathfrak{W}=V\left(\operatorname{ccl}_{\circledast}(\mathfrak{F}) ; \mathfrak{T}\right)$, $\mathfrak{I}$ being a $S_{2}$-subgroup of $\mathfrak{M}$. Let $\mathfrak{W}^{*}=\left\langle\boldsymbol{V}\left(\operatorname{ccl}_{\circledast}\left(\mathfrak{F}_{0}\right) ; \mathfrak{T}\right)|| \mathfrak{F}: \mathfrak{F}_{0} \mid=2\right\rangle$, and let $\mathfrak{X}=\Omega_{1}\left(\boldsymbol{Z}\left(\mathfrak{W}^{*}\right)\right)$.

LEMMA 13.34. One of the following holds:

(a) $N_{\mathfrak{m}}(\mathfrak{W}) \in \mathscr{L}^{*}(\mathbb{S})$.

(b) $\quad N_{\mathfrak{n}}(\mathfrak{X}) \in \mathscr{L}^{*}(\mathbb{S})$.

Proof Let $\mathfrak{S}=\boldsymbol{O}_{2}(\mathfrak{M}), \mathfrak{R}=\boldsymbol{F}(\mathfrak{M} \bmod \mathfrak{S})$. Let $\mathfrak{Q}$ be a $S_{2^{\prime}}$-subgroup of $\Re$, so that $\mathscr{D}$ is nilpotent. We first show that there is a subgroup $\mathfrak{P}$ of $\mathfrak{Q}$ such that 
(i) $\mathfrak{P}$ is a $p$-group of exponent $p$ for some prime $p \in \sigma \cup \tau$.

(ii) $\mathfrak{P}$ is non cyclic.

(iii) $\mathfrak{P} \cong \hat{\mathfrak{M}}_{p}$.

(iv) $\mathfrak{S} \mathfrak{P} \triangleleft \mathfrak{M}$.

Namely, for each prime $p$ in $\sigma \cup \tau$, let $\mathfrak{Q}_{p}$ be the $S_{p}$-subgroup of $\mathfrak{\Omega}$. Suppose $\mathfrak{D}_{p}$ contains a non cyclic characteristic abelian subgroup $\mathfrak{B}$. Take $\mathfrak{P}=\Omega_{1}(\mathfrak{B})$. Then (i), (ii), (iv) are satisfied. If $p \in \tau$, so is (iii) since every element of $\mathfrak{M}$ of order $p$ is in $\hat{\mathfrak{M}}_{p}$. If $p \in \sigma$, then (iii) holds if $m(\mathfrak{P}) \geqq 3$, while if $m(\mathfrak{P})=2$, then $C(\mathfrak{P})$ contains an elementary subgroup of order $p^{3}$, so again (iii) holds.

We may therefore assume that if $p \in \sigma \cup \tau$, then every characteristic abelian subgroup of $\mathfrak{\Omega}_{p}$ is cyclic. Hence, $\mathfrak{\Omega}_{p}=\mathfrak{Z}_{p} \Omega_{1}\left(\mathfrak{Q}_{p}\right)$, where $\mathfrak{Z}_{p}=$ $\boldsymbol{Z}\left(\mathfrak{\Omega}_{p}\right)$, and $\Omega_{1}\left(\mathfrak{\Omega}_{p}\right)$ is either extra special or of order at most $p$. If $\left|\Omega_{1}\left(\mathfrak{\Omega}_{p}\right)\right|>p^{3}$, we take $\mathfrak{P}=\Omega_{1}\left(\mathfrak{\Omega}_{p}\right)$. In this case, for each $P \in \mathfrak{P}^{\sharp}, C_{\Re}(P)$ contains an elementary subgroup of order $p^{3}$, since $\left|\Omega_{1}\left(\Omega_{p}\right)\right| \geqq p^{5}$. Thus, $\mathfrak{P}^{\sharp} \subseteq \hat{\mathfrak{M}}_{p}$, and (i)-(iv) hold. Suppose $\left|\Omega_{1}\left(\mathfrak{\Omega}_{p}\right)\right|=p^{3}$. If $p \in \tau$, we may take $\Omega_{1}\left(\mathfrak{\Omega}_{p}\right)=\mathfrak{P}$, since all elements of $\mathfrak{M}$ of order $p$ are in $\hat{\mathfrak{M}}_{p}$. Thus, in our search for $\mathfrak{P}$, we may assume that for each $p$ in $\tau, \mathfrak{\Omega}_{p}$ is cyclic, while if $p \in \sigma$, then $\mathfrak{\Omega}_{p}$ is either cyclic or is the central product of a cyclic group and an extra special group of order $p^{3}$. Let $p$ be the largest prime in $\sigma$. Since $\mathfrak{\Omega}_{q}$ is cyclic for all $q \in \tau$, it follows that a $S_{p}$-subgroup $\mathfrak{M}_{p}$ of $\mathfrak{M}$ centralizes $\mathfrak{S}_{\mathcal{C}} \mathfrak{\Omega}_{q} / \mathfrak{K}$, as $\mathfrak{M}_{p} \subseteq \mathfrak{M}^{\prime}$. By definition of $\tau$ and Lemma 13.22, it follows that $\mathfrak{M}_{p}$ centralizes $\mathfrak{S C O}_{r} / \mathfrak{S}$ for all $r \notin \sigma$. If $q \in \sigma, q \neq p$, then $p>q$, so $\mathfrak{M}_{p}$ centralizes $\mathfrak{S C O}_{q} / \mathfrak{S}$, by the special shape of $\mathfrak{D}_{q}$. Since $\mathfrak{M}_{p} \subseteq \mathfrak{M}^{\prime}$, it follows that $\mathfrak{M}_{p}$ centralizes $\mathfrak{S}_{\mathfrak{c}} \mathfrak{\Omega}_{p} / \mathfrak{S}_{\mathcal{C}} \Omega_{1}\left(\mathfrak{\Omega}_{p}\right)$ and also centralizes $\mathfrak{S}_{\mathfrak{c}} \Omega_{1}\left(\mathfrak{\Omega}_{p}\right)^{\prime} / \mathfrak{S}$, both these factors being cyclic. Hence, $\mathfrak{M}_{p} / \mathfrak{\Omega}_{p}$ is faithfully represented on $\mathfrak{S}_{2} \Omega_{1}\left(\Omega_{p}\right) / \mathfrak{S}_{2} \Omega_{1}\left(\Omega_{p}\right)^{\prime}$. Furthermore, the chain $\mathfrak{S}_{2} \Omega_{1}\left(\Omega_{p}\right)^{\prime} \subset \mathfrak{S}_{2} \Omega_{1}\left(\Omega_{p}\right)$ must be part of a chief series for $\mathfrak{M}$, since otherwise $\mathfrak{M}_{p}$ centralizes every chief factor of $\mathfrak{M}$ between $\mathfrak{K}$ and $\Re$, which in turn forces $\mathfrak{M}_{p} \subseteq \Re$, which is false. Since $\left|\Omega_{1}\left(\Omega_{p}\right): \Omega_{1}\left(\mathfrak{\Omega}_{p}\right)^{\prime}\right|=p^{2}$, we get $p=3$. Since $\mathfrak{Y}=\mathfrak{S}_{2} \Omega_{1}\left(\mathfrak{\Omega}_{3}\right) / \mathfrak{S}_{2} \Omega_{1}\left(\mathfrak{\Omega}_{3}\right)^{\prime}$ is a chief factor of $\mathfrak{M}$, the four subgroups of $\mathfrak{Y}$ of order 3 are permuted transitively by $\boldsymbol{A}_{\mathfrak{M}}(\mathfrak{Y})$, so that $\Omega_{1}\left(\Omega_{\mathfrak{3}}\right)^{\sharp} \subseteq \hat{\mathfrak{M}}_{3}$. Thus, in all cases, $\mathfrak{P}$ is available satisfying (i)-(iv).

If we now choose $\mathfrak{P}$ of least order satisfying (i)-(iv), it follows that $\mathfrak{P}$ is either elementary or extra special. So we assume in addition to (i)-(iv) that $\mathfrak{P}$ satisfies

(v) $\mathfrak{P}$ is either elementary or extra special.

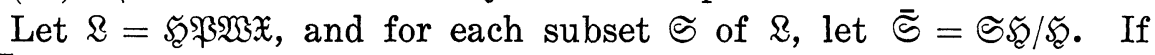
$C_{\overline{\mathfrak{B}}}(\overline{\mathfrak{W}}) \neq 1$, then $N_{\mathfrak{m}}(\mathfrak{W}) \in \mathscr{C}^{*}(\mathfrak{S})$, since $N_{\mathfrak{m}}(\mathfrak{W})$ contains an element of $\hat{\mathfrak{M}}_{p}$ in this case. So we may assume that

$$
C_{\overline{\mathfrak{\beta}}}(\overline{\mathfrak{B}})=1 \text {. }
$$

We may in addition assume that 
since otherwise (b) holds.

Let $\tilde{\mathscr{F}}=\left\{\mathfrak{F}^{G} \mid G \in \mathfrak{G}, \mathfrak{F}^{G} \subseteq \mathfrak{M},\left[\mathfrak{F}^{G}, \mathfrak{P}\right] \nsubseteq \mathfrak{S}\right\} . \quad$ By $(13.2), \tilde{\mathscr{F}} \neq \varnothing$.

The first task is to show that if $\mathfrak{F}^{*} \in \tilde{\mathscr{F}}$, then $\left|\mathfrak{F}^{*}: \mathfrak{F}^{*} \cap \mathfrak{S}\right|=2$. Since $\mathfrak{F}^{*} \in \tilde{\mathscr{F}}$, there is $G \in \mathbb{B}$ such that $\mathfrak{F}^{*}=\mathfrak{F}^{G}$. Let $\mathfrak{P}_{1}$ be a subgroup of $\mathfrak{P}$ of order $p$ such that $\mathfrak{F}_{\mathfrak{C}} \mathfrak{B}_{1}$ admits $\mathfrak{X} \mathfrak{F}^{*}$ and $\left[\mathfrak{P}_{1}, \mathfrak{F}^{*}\right] \nsubseteq \mathfrak{F}_{2}$. Note that since $\mathfrak{X}$ centralizes $\mathfrak{W}, \mathfrak{X} \mathfrak{F}^{*}$ is an elementary, so that $\mathfrak{P}_{1}$ exists. Let $\mathfrak{F}_{1}^{*}=C_{\mathfrak{F}^{*}}\left(\mathfrak{P}_{1}\right)$ so that $\mathfrak{F}_{1}^{*}$ is a hyperplane of $\mathfrak{F}^{*}$. Let $\mathfrak{X}_{1}=C_{\mathfrak{x}}\left(\mathfrak{F}_{1}\right)$, so that $\left|\mathfrak{X}: \mathfrak{X}_{1}\right| \leqq 2$. Let $\mathfrak{Y}$ be the normal closure of $\mathfrak{F}_{1}^{*}$ in $\mathfrak{S}_{\mathfrak{B}} \mathfrak{F}_{1}^{*} \mathfrak{X}$. Since (13.3) $\mathfrak{F}_{\mathfrak{C}} \mathfrak{F}_{1}^{*} \triangleleft \mathfrak{F}_{\mathfrak{C}} \mathfrak{P}_{1} \mathfrak{F}^{*} \mathfrak{X}$, we get $\mathfrak{Y} \subseteq \mathfrak{T}$. Hence, $\mathfrak{Y}$ is generated by conjugates of $\mathfrak{F}_{1}^{*}$, so $\mathfrak{Y} \subseteq \mathfrak{W}^{*}$. Hence, $\mathfrak{X}$ centralizes $\mathfrak{Y}$. If $\mathfrak{X}_{1} \subset \mathfrak{X}$, then $C\left(\mathfrak{Y )}\right.$ contains $\mathfrak{P}_{1}$. But $\mathfrak{F}^{* \sharp} \subseteq \mathcal{J}^{G}$, so that $C(\mathfrak{Y}) \subseteq \mathfrak{M}^{G}$. This is impossible, since $\left[\mathfrak{F}^{*}, \mathfrak{P}_{1}\right]$ is not a 2-group. Hence, $\mathfrak{X}_{1}=\mathfrak{X}$.

Let $\mathfrak{S}^{*}=\boldsymbol{C}(\mathfrak{Y}) \cap \mathfrak{S}_{\mathfrak{F}}^{*} \mathfrak{X}$, so that $\mathfrak{C}^{*}$ is a normal 2-subgroup of $\mathfrak{S}_{\mathfrak{1}} \mathfrak{F}_{1}^{*} \mathfrak{X}_{1}$ which contains $\mathfrak{X}$. Also, $\mathfrak{C}^{*} \subseteq \mathfrak{M}^{G}$, since $\mathfrak{F}_{1}^{*} \neq 1$, and $\mathfrak{F}^{* \#} \subseteq \mathscr{J}^{G}$. Choose $F \in \mathfrak{F}^{*}-\mathfrak{F}_{1}^{*}$. Then $F$ inverts an element $P$ of $\mathfrak{S}_{\mathfrak{F}} \mathfrak{F}^{*} \mathfrak{X} \mathfrak{P}_{1}$ of order $p$. Let $\mathfrak{D}=\langle P, F\rangle$, so that $\mathfrak{D}$ is dihedral of order $2 p$. Let $\mathfrak{E}_{1}^{*}=$ $\left[\mathfrak{S}^{*},\langle P\rangle\right]$, so that $\mathfrak{D}$ normalizes $\mathfrak{C}_{1}^{*}$, and $\mathfrak{S}_{1}^{*} \subseteq \mathfrak{M}^{\sigma}$. Let $\mathfrak{S}_{2}^{*}=\left[\mathfrak{S}_{1}^{*}, F\right]$. Since (13.3) holds, we have $\mathfrak{S}_{1}^{*} \neq 1$, and so $\mathfrak{C}_{2}^{*} \neq 1$. Since $\mathfrak{S}_{1}^{*} \subseteq \mathfrak{M}^{G}$, we get $\mathfrak{C}_{2}^{*} \subseteq \mathfrak{F}^{a}$. Since $\mathfrak{C}^{*} \cap \mathfrak{F}^{G} \neq 1$, it follows that $C\left(\mathfrak{C}^{*}\right) \subseteq \mathfrak{M}^{G}$, as $\mathfrak{F}^{* *} \cong \mathscr{J}^{G}$. Let $\mathfrak{U}=C\left(\mathfrak{C}^{*}\right) \cap \mathfrak{F}_{\mathfrak{C}} \mathfrak{F}_{1}^{*} \mathfrak{X}$, so that $\mathfrak{D}$ normalizes $\mathfrak{u}$, $\mathfrak{F}_{1}^{*} \subseteq \mathfrak{Y} \subseteq \mathfrak{U}$, and $\mathfrak{u} \subseteq$ $\mathfrak{M}^{G}$. Let $\mathfrak{U}^{*}=[\mathfrak{u},\langle P\rangle]$, so that $\mathfrak{U}^{*} \neq 1$, since $\mathfrak{P}_{1} \nsubseteq \mathfrak{M}^{G}$. Let $\mathfrak{U}_{1}^{*}=$ $\left[\mathfrak{u}^{*}, F\right]$. Since $\mathfrak{u}^{*} / \boldsymbol{D}\left(\mathfrak{u}^{*}\right)$ is a free $F_{2}\langle F\rangle$-module, it follows that $\mathfrak{U}^{*}=\left\langle\mathfrak{U}_{1}^{*}, \mathfrak{U}_{1}^{*^{P}}\right\rangle$. Now $\mathfrak{U}_{1}^{*}$ is a normal elementary subgroup of $\mathfrak{U}^{*}$ contained in $\mathfrak{F}^{*}$, so $\mathfrak{U}^{*}=\mathfrak{U}_{1}^{*} \mathfrak{U}_{1}^{*^{P}}, \mathfrak{U}^{* \prime}=\left[\mathfrak{u}_{1}^{*}, \mathfrak{U}_{1}^{*^{P}}\right] \subseteq \mathfrak{F}^{*}$. Since $F$ centralizes $\mathfrak{F}^{*}$, we get that $F$ centralizes $\mathfrak{u}^{* \prime}$, so $P$ centralizes $\mathfrak{u}^{* \prime}$. Since $\mathfrak{U}^{* \prime} \subseteq \mathfrak{F}^{*}$, we get $\mathfrak{U}^{* \prime}=1$, so that $\mathfrak{u}^{*}=\mathfrak{U}_{1}^{*} \times \mathfrak{U}_{1}^{* P}$.

Since $P$ centralizes $\mathfrak{u} / \mathfrak{U}^{*}$, we get $\mathfrak{U}=\mathfrak{U}^{*} C_{\mathfrak{u}}(P), \mathfrak{u}^{*} \cap C(P)=1$. Since $F$ normalizes $C_{\mathfrak{u}}(P)$ and since $P \notin \mathfrak{M}^{G}$, we get

$$
\left[F, C_{\mathfrak{u}}(P)\right] \subseteq C_{\mathfrak{u}}(P) \cap \mathfrak{F}^{G}=1 .
$$

Choose $F^{*} \in \mathfrak{F}_{1}^{*}$. Then $F^{*}=A B$, where $A \in C_{\mathfrak{u}}(P), B \in \mathfrak{U}^{*}$. Since $F$ centralizes $F^{*}$ and $A$, we get $B \in C_{\mathfrak{u}^{*}}(F)=\mathfrak{U}_{1}^{*}$. Hence, $A \in \mathfrak{F}_{1}^{*}$, so that $A=1$, as $P \notin \mathfrak{M}^{G}$. Thus, $\mathfrak{u}_{1}^{*}=\mathfrak{F}_{1}^{*} \subseteq \mathfrak{U}^{*}=[\mathfrak{u}, P] \subseteq \mathfrak{S}_{\mathcal{L}}$, so we get the desired conclusion:

$$
\mathfrak{F}^{G} \in \tilde{\mathscr{F}} \text { implies }\left|\mathfrak{F}^{G}: \mathfrak{F}^{G} \cap \mathfrak{S}\right|=2 \text {. }
$$

Retaining our notation $\mathfrak{F}^{*}=\mathfrak{F}^{a} \in \tilde{\mathscr{F}}$, let

$$
\tilde{\mathfrak{B}}=\left\langle V\left(\operatorname{ccl}_{\mathfrak{B}}\left(\mathfrak{F}_{0}\right) ; \mathfrak{S}\right)|| \mathfrak{F}: \mathfrak{F}_{0} \mid=2\right\rangle \text {. }
$$

Thus, $\tilde{\mathfrak{S}} \cong \mathfrak{S}^{*}$ and $\mathfrak{F}_{1}^{*}=\mathfrak{F}^{*} \cap \mathfrak{S} \subseteq \tilde{\mathfrak{B}}$, since $\mathfrak{F}_{1}^{*} \in \operatorname{ccl}_{\mathfrak{E}}\left(\mathfrak{F}_{0}\right)$ for some subgroup $\mathfrak{F}_{0}$ of index 2 in $\mathfrak{\mho}$. 
Since $\tilde{\mathfrak{B}} \triangleleft \mathfrak{M}$, so also $\tilde{\mathfrak{S}} \triangleleft \mathfrak{S} \mathfrak{P} \mathfrak{M X}=\mathfrak{R}$. Now $\mathfrak{S}^{*} \cong \hat{\mathfrak{M}}_{p}$, so that $\mathfrak{B} \cap \mathfrak{M}^{(x}=1$. Hence, $C_{\mathfrak{R}}(\tilde{\mathfrak{S}})$ is a 2-group with $\mathfrak{X} \subseteq C_{\mathfrak{R}}(\tilde{\mathfrak{S}})$. Let $\tilde{\mathfrak{Y}}=$ $C_{\mathfrak{R}}(\tilde{\mathfrak{B}})$, so that $\tilde{\mathfrak{Y}} \triangleleft \mathbb{R}$, and $\tilde{\mathfrak{Y}} \subseteq \mathfrak{M}^{a}$. Let $\mathfrak{B}_{0}$ be a subgroup of $\mathfrak{P}$ of order $p^{2} ; \mathfrak{F}_{0}$ is available by our construction of $\mathfrak{\beta}$. Choose $F \in \mathfrak{F}^{G}-\mathfrak{F}^{G} \cap \mathfrak{S}$. For each $A$ in $\mathfrak{P}^{\sharp}$, we get $C_{\mathfrak{y}}(A) \cap \mathfrak{F}^{a}=1$, since $A \notin \mathfrak{M}^{a}$.

Now $\tilde{\mathfrak{Y}} \subseteq \mathfrak{M}^{\alpha}$ and $\tilde{\mathfrak{Y}}$ centralizes the hyperplane $\mathfrak{F}_{1}^{*}$ of $\mathfrak{F}^{(*}$. Furthermore, $[\tilde{\mathfrak{Y}}, F] \in \tilde{\mathfrak{Y}} \cap \widetilde{\mathfrak{F}}_{1}^{*}$. But $\tilde{\mathfrak{Y}}$ centralizes $\tilde{\mathfrak{B}}$ and $\mathfrak{F}_{1}^{*} \subseteq \tilde{\mathfrak{Y}}$, so $\tilde{\mathfrak{Y}} \cap \mathfrak{F}_{1}^{*} \subseteq$ $\Omega_{1}(\boldsymbol{Z}(\tilde{\mathfrak{Y}}))$. Hence, $F$ centralizes $\tilde{\mathfrak{Y}} / \Omega_{1}(\boldsymbol{Z}(\tilde{\mathfrak{Y}}))$, and since $\tilde{\mathfrak{Y}}$ centralizes the hyperplane $\mathfrak{F}_{1}^{*}$ of $\mathfrak{F}^{G}, F$ centralizes $D(\tilde{\mathfrak{Y}})$. By (13.2), it follows that $\mathfrak{P}$ centralizes $\tilde{\mathfrak{Y}} / \Omega_{1}(\mathbb{Z}(\tilde{\mathfrak{Y}}))$ and $\boldsymbol{D}(\tilde{\mathfrak{Y}})$. Thus, $\mathfrak{P}$ is faithfully represented on $\Omega_{1}(Z(\tilde{\mathfrak{Y}}))=\mathfrak{U}$, say.

Let $P$ be an element of $\mathfrak{S} \mathfrak{B M X}$ of order $p$ inverted by $F$. Thus, setting $\widetilde{\mathbb{S}}=\left\{X \in \mathfrak{S} \mathfrak{P} \mathfrak{W} \mid P^{x}=P\right.$ or $\left.P^{-1}\right\}$, we see that $\widetilde{\mathbb{S}}$ normalizes $\mathfrak{B}=[\mathfrak{A}, P]$ and $\widetilde{\mathbb{C}}$ contains $F$. Let $\mathfrak{B}_{1}$ be a minimal normal subgroup of $\widetilde{\mathfrak{S}}$ with $\mathfrak{B}_{1} \subseteq \mathfrak{B}$. Thus, $\langle P\rangle$ is faithfully represented on $\mathfrak{B}_{1}$, as $P$ has no non trivial fixed points on $\mathfrak{B}$. Hence, $\mathfrak{B}_{1}$ is a free $F_{2}\langle F\rangle$-module, so $\mathfrak{B}_{1} \cap \mathfrak{F}^{G} \neq 1$. Hence, a $S_{p}$-subgroup of $\widetilde{\mathbb{C}}$ is non cyclic and is faithfully represented on $\mathfrak{B}_{1}$. By minimality of $\mathfrak{B}_{1}, \mathfrak{S} \cap \widetilde{\mathfrak{S}}$ centralizes $\mathfrak{B}_{1}$, and so $\boldsymbol{A}_{\widetilde{\mathbb{E}}}\left(\mathfrak{B}_{1}\right)$ is $p$-closed. Let $F_{0}$ be the image of $F$ in $\boldsymbol{A}_{\tilde{\mathbb{\varepsilon}}}\left(\mathfrak{B}_{1}\right)$ and let $\mathfrak{P}_{0}$ be a $S_{p}$-subgroup of $\boldsymbol{A}_{\widetilde{\mathbb{E}}}\left(\mathfrak{B}_{1}\right)$. Thus, $F_{0}$ normalizes $\mathfrak{P}_{0}$ and $\mathfrak{P}_{0}$ is non cyclic. Suppose $\mathfrak{P}_{0} \cap C\left(F_{0}\right)=\mathfrak{P}_{00} \neq 1$. Then $\mathfrak{P}_{00}$ is faithfully represented on $\mathfrak{C}_{\mathfrak{B}_{1}}\left(F_{0}\right)$, by the $\mathfrak{S} \times \mathfrak{Q}$-lemma. But $C_{\mathfrak{\vartheta}_{1}}(F)=\left[\mathfrak{B}_{1}, F\right] \subseteq \mathfrak{M}^{\alpha}$. Since $\mathfrak{F}^{G \sharp} \subseteq \mathscr{J}^{G}$, it follows that $N\left(C_{\mathfrak{R}_{1}}(F)\right) \subseteq \mathfrak{M}^{G}$. Since $\mathfrak{S} \mathfrak{P} \cap \mathfrak{M}^{G}$ is a 2-group, we have $\mathfrak{P}_{00}=1$. Hence, $F_{0}$ inverts $\mathfrak{P}_{0}$. Choose $P_{0} \in \mathfrak{P}_{0}^{*}$. Then $\mathfrak{B}_{1} \cap$ $\mathcal{C}\left(P_{0}\right)$ admits $F_{0}$ 。 Since $\mathfrak{S} \mathfrak{P} \cap \mathfrak{M}^{G}$ is a 2-group, it follows that $\mathfrak{B}_{1} \cap$ $C\left(P_{0}\right) \cap \mathfrak{F}^{F}=1$. Since $\left[\mathfrak{B}_{1} \cap \boldsymbol{C}\left(P_{0}\right), F^{\prime}\right] \subseteq \mathfrak{F}^{G}$, it follows that $F$ centralizes $\mathfrak{B}_{1} \cap C\left(P_{0}\right)$. Since $\mathfrak{B}_{1}=\left\langle\mathfrak{B}_{1} \cap C\left(P_{0}\right) \mid P_{0} \in \mathfrak{P}_{0}^{*}\right\rangle$, it follows that $F$ centralizes $\mathfrak{B}_{1}$. This is not the case, since $\mathfrak{B}_{1}$ is a free $F_{2}\langle F\rangle$-module. The proof is complete.

Lemma 13.35. Suppose the following hold for some $G$ in (S;

(a) $\left|\mathfrak{F}^{G}: \mathfrak{F}^{G} \cap \mathfrak{M}\right|=2$.

(b) $\mathfrak{F}^{G} \cap \mathfrak{M}$ centralizes a hyperplane of $\mathfrak{F}$.

Then one of the following holds:

(i) $|\mathfrak{F}|=8$.

(ii) $|\mathfrak{F}|=16,|\mathfrak{F}|=4$.

Proof. Let $\mathfrak{A}=\mathfrak{F}^{G} \cap \mathfrak{M}, \mathfrak{F}_{0}=\mathfrak{F} \cap C(\mathfrak{Z})$, so that $\mathfrak{F}_{0}$ contains a hyperplane of $\mathfrak{F}$. If $\mathfrak{F} \subseteq \mathfrak{M}^{G}$, then by Lemma 13.30 applied to $\mathfrak{M}^{G}$, we get $\mathfrak{F} \subseteq C\left(\mathfrak{F}^{G}\right)$, and so $\mathfrak{F}^{G} \subseteq \mathfrak{M}$, against our assumption. Hence, $\mathfrak{F} \nsubseteq \mathfrak{M}^{G}$, and so $\mathfrak{F}_{0}$ is hyperplane of $\mathfrak{F}$. Since $C(X) \subseteq \mathfrak{M}^{G}$ for every $X$ in $\mathfrak{F}^{G \sharp}$, it follows that $\mathfrak{F}_{0}=C_{\widetilde{\Im}}(X)$ for every $X \in \mathfrak{A}^{\sharp}$. By Lemma 13.22, $|\mathfrak{A}: \mathfrak{N} \cap \mathfrak{D}| \leqq 2$. Since $\mathfrak{A} \cap \mathfrak{D}$ acts faithfully on $\mathfrak{F}$ and stabilizes the chain $\mathfrak{F} \supset \mathfrak{F} \supset 1$, we get $|\mathfrak{A} \cap \mathfrak{D}| \leqq|\mathfrak{F}|$, whence 


$$
\left|\mathfrak{F}^{G}\right|=2|\mathfrak{A}| \leqq 2^{2}|\mathfrak{A} \cap \mathfrak{D}| \leqq 2^{2}|\mathfrak{F}| \text {. }
$$

Since $|\mathfrak{F}| \leqq 2^{2}$, the proof is complete.

\section{LEMMA 13.36. If $\mathfrak{I} \subseteq \mathbb{S} \in \mathscr{S} \mathscr{O} \mathscr{L}(\mathfrak{S})$, then $\mathfrak{F} \subseteq \boldsymbol{O}_{2}(\mathfrak{S})$.}

Proof. Suppose false, and that $\subseteq$ is a minimal counterexample. Thus, $\subseteq=\mathfrak{T} \mathfrak{S}$ for some $q$-group $\mathfrak{\Omega}$. By Lemma 13.20, $\mathscr{S} \mathscr{C} \mathscr{N}_{3}(\mathfrak{l})=\varnothing$. Let $\mathfrak{S}=\boldsymbol{O}_{2}(\mathfrak{S})$. By minimality of $\mathfrak{S}$, we get $\mathfrak{S} \mathfrak{\Omega} \triangleleft \mathfrak{S}$, and $\mathfrak{F} \subseteq \boldsymbol{O}_{2}(\mathfrak{T} \boldsymbol{D}(\mathfrak{Q}))$, while $\mathfrak{S} \mathfrak{\Omega} / \mathfrak{S} D(\mathfrak{Q})$ is a chief factor of $\mathfrak{S}$.

First, suppose that $\mathfrak{Q}$ is cyclic. In this case, $\mathfrak{F} / \mathfrak{F}$ is cyclic and $\mathfrak{F} \cap \mathfrak{F}=\mathfrak{F}_{0}$ is of index 2 in $\mathfrak{F}$. Choose $F \in \mathfrak{F}-\mathfrak{F}_{0}$. Then we may assume that $F$ inverts a generator $Q$ for $\cong$. Since $\mathfrak{F} \leqq O_{2}(\mathfrak{T} D(\mathfrak{\Omega}))$, we get $\left|\mathfrak{D}_{1}\right|=q$.

Let $\mathscr{S}_{0}=\left[\mathfrak{S}_{\mathfrak{c}}, \mathfrak{D}_{\mathrm{D}}\right]$. Thus, $\mathfrak{S}_{0} / \boldsymbol{D}\left(\mathfrak{S}_{0}\right)$ is a free $F_{2}\langle F\rangle$-module. Let $\mathfrak{S}_{1}=\left[\mathfrak{S}_{0}, F\right]$, so that $\mathfrak{S}_{0}=\mathfrak{S}_{1} \cdot \mathfrak{S}_{1}^{Q}$, and $\mathfrak{S}_{1} \subseteq \mathfrak{F}$. Hence, $\mathfrak{S}^{\prime}=\left[\mathfrak{S}_{1}, \mathfrak{S}_{1}^{Q}\right]$ is centralized by $F$, so is centralized by $Q$. Since $Q \notin \mathfrak{M}$, and $\mathfrak{F}^{\sharp} \subseteq \mathscr{J}$, it follows that $\mathfrak{S}_{0}^{\prime}=1$.

Let $\mathfrak{S}_{2}=C_{\mathfrak{g}}(Q)$, so that $\mathfrak{S}_{\mathrm{S}}=\mathfrak{S}_{2} \mathfrak{S}_{2}, \mathfrak{S}_{2} \cap \mathfrak{S}_{0}=1$. Since $\left[\mathfrak{S}_{2}, F\right] \subseteq$ $\mathfrak{S}_{2} \cap \mathfrak{F}=1$, the equality holding since $Q \notin \mathfrak{M}$ and $\mathfrak{F}^{\sharp} \subseteq \mathscr{F}$, we get that for each $F_{0}$ in $\mathfrak{F}_{0}, F_{0}=A B$, where $A \in \mathfrak{S}_{2}, B \in \mathfrak{S}_{0}$. Since $F$ centralizes $F_{0}$ and $A, F$ centralizes $B$, so $B \in \mathfrak{S}_{1}=C_{\mathfrak{p}_{0}}(F)$, which then gives $A \in \mathfrak{S}_{2} \cap \mathfrak{F}=1$. Hence, $\mathfrak{F}_{0}=\mathscr{S}_{1}$ is a hyperplane of $\mathfrak{F}$. Let $\mathfrak{B}=\mathfrak{S}_{1}^{Q}$, so that $\mathfrak{F}^{Q} \cap \mathfrak{M}=\mathfrak{B}$, and $\mathfrak{B}$ centralizes the hyperplane $\mathfrak{S}_{1}$ of F. By Lemma 13.35, it follows that $|\mathfrak{F}| \leqq 16$.

Suppose $|\mathfrak{F}|=8$. Here, we get $\left|\mathfrak{S}_{0}\right|=16, \mathfrak{S}_{0}=\mathfrak{S}_{1} \times \mathfrak{S}_{1}^{Q}$, and $\left|\mathscr{S}_{1}\right|=4$. Hence, $q=3$ or 5 . Let $\mathfrak{S}_{3}=\mathfrak{S}_{2} \cap \boldsymbol{C}\left(\mathfrak{S}_{0}\right)$. Then $\mathfrak{S}_{3} \triangleleft \mathfrak{S}$. If $\mathfrak{S}_{3} \neq 1$, then $\mathfrak{S}_{3} \cap Z(\mathfrak{I}) \neq 1$, against $\Omega_{1}(Z(\mathfrak{I}))^{\#} \subseteq \mathscr{J}$, and $Q \notin \mathfrak{M}$. Hence, $\mathfrak{S}_{3}=1$. Since $Q$ centralizes $\mathfrak{S}_{2}$, it follows that $\mathfrak{S}_{2}$ is faithfully represented on $\mathfrak{K}_{1}$. Hence, $\left|\mathfrak{S}_{2}\right| \leqq 2$, as $\left|\mathfrak{S}_{1}\right|=4$. If $q=3$, we get $|\mathfrak{T}| \leqq 64$. Since $\boldsymbol{O}_{2^{\prime}}(\mathfrak{M})=1$ and $\mathfrak{M}$ contains an elementary subgroup of order $p^{3}$ for some odd prime $p$, we get $\mathfrak{I}=\boldsymbol{O}_{2}(\mathfrak{M}), p=3$, and $\mathfrak{I}$ is an elementary. This is clearly not the case, since $l_{2}(S)=2$. If $q=5$,

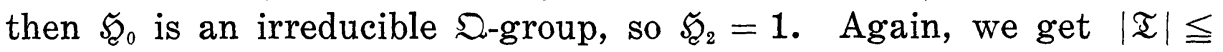
64 , a contradiction.

Suppose $|\mathfrak{F}|=16$. Here we get $q=3$ or 7 , since $\left|\mathfrak{S}_{0}\right|=64$, and since $\mathfrak{\Omega}$ has no non trivial fixed points on $\mathfrak{S}_{0}$. If $q=7$, then $\mathfrak{S}_{0}$ is a minimal normal subgroup of $\mathfrak{S}$, so that $\mathfrak{S}_{2}=1,|\mathfrak{T}|=128$. Since $\mathfrak{M}$ has an elementary subgroup $\mathfrak{P}$ of order $p^{3}$ for some odd prime $p$, and since $\mathfrak{P}$ is represented faithfully on $\boldsymbol{O}_{2}(\mathfrak{M})$, it follows that either $\boldsymbol{O}_{2}(\mathfrak{M})$ is an elementary of order 64 , or $\boldsymbol{O}_{2}(\mathfrak{M})=\mathfrak{T}$. The first case yields $\boldsymbol{O}_{2}(\mathfrak{M})=\boldsymbol{O}_{2}(\mathfrak{S})$, against $\mathfrak{S} \nsubseteq \mathfrak{M}$. The second case is impossible since $\mathfrak{T}^{\prime}=\mathfrak{S}_{1}$ is of order 8 .

It remains to treat the case $|\mathfrak{F}|=16, q=3$. Here we get $\mathfrak{I}=$ 
$\mathfrak{S}_{\mathfrak{S}}\langle F\rangle$. Since $\mathfrak{K}_{2}$ is faithfully represented on $\mathfrak{F}_{1}$, and since $\mathfrak{S}_{\mathfrak{C}} \cap C(F)=$ $\mathfrak{S}_{2} \mathfrak{S}_{1}$, it follows that $\mathfrak{F}=\mathfrak{S}_{1} \times\langle F\rangle$ is a self-centralizing normal subgroup of $\mathfrak{T}$. Consider $\mathrm{O}_{2}(\mathfrak{M})$. Since $|\mathfrak{S}|=4$, and $\mathfrak{F} / \mathfrak{F}$ is a chief factor of $\mathfrak{M}$, it follows that $\left[\mathfrak{F}, \boldsymbol{O}_{2}(\mathfrak{M})\right] \subseteq \mathfrak{G}$. Since $\mathfrak{M}$ contains an elementary subgroup of order $p^{3}$ for some odd prime $p$, it follows that $M$ contains a subgroup $\mathfrak{P}$ of order $p$ which centralizes $\mathfrak{F}$. Hence, $\left[\boldsymbol{O}_{2}(\mathfrak{M}), \mathfrak{P}\right] \subseteq$ $\boldsymbol{C}(\mathfrak{F}) \cap \boldsymbol{O}_{2}(\mathfrak{M})=\mathfrak{F}$, so $\mathfrak{F}$ stabilizes $\boldsymbol{O}_{2}(\mathfrak{M}) \supset \mathfrak{F} \supset 1$. This is impossible since $O_{2}(\mathfrak{M})=1$.

We may now assume that $\mathfrak{\Omega}$ is non cyclic. Let $\mathfrak{\Omega}_{1}$ be a subgroup of $\Omega$ of order $q$ such that $\mathfrak{S}_{\mathfrak{C}} \mathfrak{\Omega}_{1}$ admits $\mathfrak{F}$ and $\left[\mathfrak{F}, \mathfrak{D}_{1}\right] \nsubseteq \mathfrak{F}$. Let $\mathfrak{S}_{1}=$ $\mathfrak{S}_{\mathfrak{S}} \mathfrak{Q}_{1} \mathfrak{F}, \tilde{\mathfrak{F}}=\boldsymbol{O}_{2}\left(\mathfrak{S}_{1}\right)$, and $\mathfrak{F}_{1}=\mathfrak{F} \cap \tilde{\mathfrak{F}}$. Then $\left|\mathfrak{F}: \mathfrak{F}_{1}\right|=2$. Choose $F \in$ $\mathfrak{F}-\widetilde{\mho}_{1}$. We assume without loss of generality that $F$ inverts a

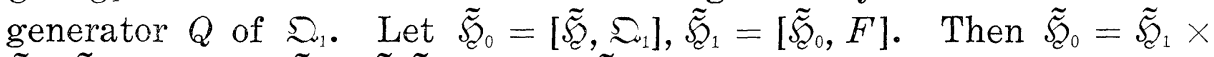

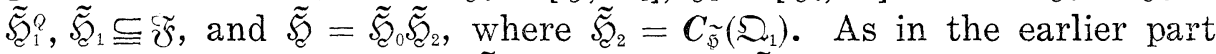

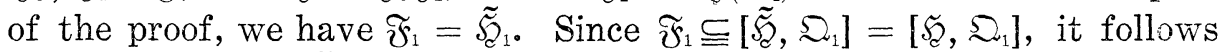
that $\widetilde{F}_{1} \subseteq \mathfrak{S}, \mathfrak{F}_{\mathrm{C}}=\tilde{\mathfrak{F}}$. Hence, $\widetilde{F} \mathfrak{F} / \mathscr{S C}$ is a central subgroup of $\mathfrak{I} / \mathfrak{S C}$ of order 2. Since $\mathfrak{S} \mathfrak{S} / \mathfrak{S C D}(\mathfrak{C})$ is a chief factor of $\mathfrak{S}$, it follows that $\mathfrak{F}$ inverts $\mathscr{S} \cong / / \mathscr{E} D(\Omega)$. Since $F$ centralizes $\mathfrak{S} D(\Omega) / \mathscr{S}$, it follows that $\mathfrak{\Omega}$ is either non cyclic of order $q^{2}$ or extra special of order $q^{3}$ and exponent $q$.

Suppose $\mathfrak{O}$ is abelian. In this case, since $F$ inverts $\mathscr{F} \mathfrak{C} / \mathfrak{F}$, we may assume that $F$ inverts $\mathfrak{\Omega}$. Thus, $C_{\mathfrak{s}}\left(Q_{1}\right)$ admits $F$ for all $Q_{1} \in \mathfrak{D}$. Since $\mathfrak{\Omega} \cap \mathfrak{M}=1$, it follows that $\mathfrak{F} \cap C_{\mathfrak{F}}\left(Q_{1}\right)=1$ for all $Q_{1} \in \mathfrak{D}$. Hence, $\left[C_{\mathfrak{S}}\left(Q_{1}\right), F\right] \leqq \widetilde{F} \cap \boldsymbol{C}_{\mathfrak{5}}\left(Q_{1}\right)=1$. Since $\mathfrak{\mathcal { E }}=\left\langle\boldsymbol{C}_{\mathfrak{5}}\left(Q_{1}\right) \mid Q_{1} \in \mathfrak{\Omega}^{\sharp}\right\rangle$, we get that $F$ centralizes $\mathfrak{S}$. This is not the case, so $\mathfrak{D}$ is extraspecial.

Let $\mathfrak{S}_{1}=C_{\mathfrak{\Xi}}\left(\mathfrak{D}_{1}\right)$. Thus, $S_{p}$-subgroups of $\mathfrak{S}_{1}$ are of order $q^{2}$. Also, $\widetilde{S}_{1}$ normalizes $\tilde{\mathscr{F}_{0}}=\left[\tilde{\mathfrak{C}}, \Omega_{1}\right]=\left[\mathfrak{S}_{\mathcal{C}}, \mathfrak{S}_{1}\right]$. Let $\mathfrak{S}_{2}$ be a $S_{q}$-subgroup of $\mathfrak{S}_{1}$. Thus, $F$ normalizes $\mathfrak{S C O}_{2}$, and $F$ centralizes $\mathfrak{S}_{2} \mathfrak{D}_{2} / \mathfrak{S}_{2} \mathfrak{D}_{1}$. We may assume that notation is chosen so that $\mathfrak{\Omega}_{2} \subseteq \Omega$. Then $\mathfrak{\Omega}_{2}=\mathfrak{\Omega}_{1} \times \mathfrak{\Omega}^{\prime}$. Thus, $F$ centralizes $\mathfrak{S} \mathfrak{D}^{\prime} / \mathfrak{S}$. We will show that $\mathfrak{Q}^{\prime} \subseteq \mathfrak{M}$. Namely, $\mathfrak{S} \mathfrak{Q}^{\prime}\langle F\rangle$ normalizes $\tilde{\mathfrak{K}}_{0}$. Let $\mathfrak{S}_{00}$ be a minimal normal subgroup of $\mathfrak{S}_{\mathfrak{C}} \mathfrak{Q}^{\prime}\langle F\rangle$ with $\tilde{\mathscr{S}}_{00} \subseteq \tilde{\mathscr{F}}_{0}$. Since $C_{\tilde{\mathfrak{F}}_{0}}(F)=\widetilde{F}_{0}$, we are done in case $\mathfrak{Q}^{\prime}$ centralizes $\tilde{\mathfrak{F}}_{00}$, so suppose that $\left[\tilde{\mathfrak{F}}_{00}, \mathfrak{Q}^{\prime}\right] \neq 1$. Since $\mathfrak{S}_{\mathfrak{C}} \mathfrak{Q}\langle F\rangle$ is 2 -closed, we get

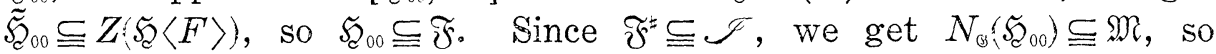
$\mathfrak{\Omega}^{\prime} \subseteq \mathfrak{M}$, as required.

Suppose $q \geqq 7$. Here we get $\left|\mathfrak{F}_{1}\right|=8,|\mathfrak{F}|=16,|\mathfrak{F}|=4$, by Lemma 13.35. Thus, $\boldsymbol{A}_{\mathfrak{n}}(\mathfrak{F})$ is a 2,3 -group, so $\mathfrak{Q}^{\prime}$ centralizes $\mathfrak{F}$. Hence, $\mathfrak{Q}$ centralizes $\mathfrak{S}_{0}$. This is not the case, since $\mathfrak{S}_{0}=\left[\mathfrak{S}_{\mathfrak{L}}, \mathfrak{\Omega}_{1}\right]$ and $\mathfrak{\Omega}$ is an extra special group which acts faithfully on $\mathfrak{S}$. Hence, $q=3$ or 5 . If $q=5$, we get $\left|\widetilde{\mathfrak{F}}_{1}\right|=4,\left|\mathfrak{F}_{0}\right|=16$, so that $\mathfrak{\Omega}^{\prime}$ centralizes $\mathfrak{S}_{0}$. This is impossible, since $\mathfrak{S}_{0}=\left[\mathfrak{S}_{\mathfrak{C}}, \mathfrak{Q}_{1}\right]$ and $\mathfrak{\Omega}$ is represented faithfully on S. Hence, $q=3$.

Let $\Re=\left[\mathfrak{S}_{2}, \Omega^{\prime}\right]$, so that $\Re \triangleleft \subseteq$. Since $\Omega$ is represented faithfully on $\Re / D(\Re)$, and $\mathfrak{Q}^{\prime}$ has no fixed points on $\Re-\boldsymbol{D}(\Re)$, it follows that 
$|\Re: D(\Re)|=2^{6 k}$, with $k \geqq 1$. If $k \geqq 2$, we get that

$$
\left|\left[\Re, \mathfrak{Q}_{1}\right] D(\Re): D(\Re)\right| \geqq 2^{8},
$$

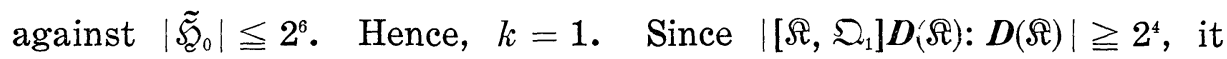
follows that $|\mathfrak{F} \cap D(\Re)| \leqq 2$. Hence, $\mathfrak{Q}^{\prime}$ centralizes $D(\Re)$, since otherwise $\left|\left[\mathfrak{\Omega}_{1}, \boldsymbol{D}(\Re)\right] \boldsymbol{D}(\boldsymbol{D}(\Re)): \boldsymbol{D}(\boldsymbol{D}(\Re))\right| \geqq 2^{4}$. Suppose $\boldsymbol{D}(\Re) \neq 1$. Since $\mathfrak{\Omega}^{\prime}$ is a $S_{3}$-subgroup of $\mathfrak{M} \cap \mathcal{S}$, it follows that $\boldsymbol{C}(\boldsymbol{D}(\mathfrak{\Re})) \cap \mathfrak{Q}=\mathfrak{Q}$. Since $\mathfrak{S C} / \mathscr{S C} D(\mathfrak{Q})$ is a chief factor of $\mathfrak{S}$, it follows that $\mathfrak{F} \cdot F$ is a square in $\mathfrak{I} / \mathfrak{S}$. This implies that $\left|\boldsymbol{D}(\Re): \boldsymbol{D}(\Re) \cap \boldsymbol{C}\left(F^{\prime}\right)\right| \geqq 4$, so that $|[\boldsymbol{D}(\Re), \mathfrak{F}]| \geqq 4$, against $|\boldsymbol{D}(\Re) \cap \mathfrak{F}| \leqq 2$. Hence, $D(\Re)=1$. Hence, $|\mathfrak{F} \cap \Re| \geqq 4$, so that $\widetilde{F}$ centralizes a subgroup of $\mathfrak{S} / \Re$ of index at most 2 . This implies that $\cong$ centralizes $\mathfrak{F} / \Re$, so that $\mathfrak{F}=\Re \Omega_{0}$, where $\Re_{0}$ centralizes $\Re$, so $\mathfrak{S}=\Re \times \Re_{0}$. Since $\Re_{0} \cap \mathfrak{F}=1$, and $\Re_{0} \triangleleft \mathfrak{S}$, we get $\Re_{0}=1$.

We next argue that $3 \notin \sigma$. Suppose false. Then since $|\mathfrak{Q}|=3^{3}$

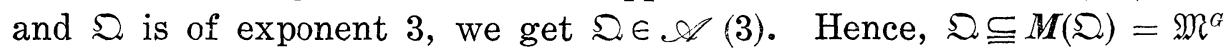
for some $G$ in $\mathbb{S}$, the equality holding by Lemma 13.20. Since $\mathfrak{D} \nsubseteq \mathbb{M}$, we have $\mathfrak{M} \neq \mathfrak{M}^{a}$. On the other hand, we have already shown that $\mathfrak{Q}^{\prime} \subseteq \mathfrak{M}$, and so $S_{3}$-subgroups of $\boldsymbol{C}_{\mathfrak{m}}\left(\Omega^{\prime}\right)$ are not cyclic. This violates Theorem 13.1. Hence, $3 \notin \sigma$. Since $\sigma \neq \varnothing$, it follows that

$$
\left|\boldsymbol{O}_{2}(\mathfrak{M}): \boldsymbol{D}\left(\boldsymbol{O}_{2}(\mathfrak{M})\right)\right| \geqq 2^{9} .
$$

Since $\mathfrak{F}=\mathscr{R}$ is of order $2^{6}$, and since $\mathfrak{I} / \mathfrak{F}$ acts faithfully on $\mathfrak{S} \cong / \mathfrak{F}$, it follows that every subgroup of $\mathfrak{I}$ can be generated by 6 elements. This contradiction completes the proof.

LEMma 13.37. Suppose $\mathfrak{S}_{0} \in \mathscr{S} \mathcal{O} \mathscr{L}(\mathbb{S})$ and the following hold:

(a) $O_{2^{\prime}}\left(\mathfrak{S}_{0}\right)=1$.

(b) $\mathfrak{\mho} \subseteq \boldsymbol{O}_{2}\left(\Im_{0}\right)$.

(c) $\mathfrak{I} \cap \mathfrak{S}_{0}=\mathfrak{S}_{2}$ is a $S_{2}$-subgroup of $\mathfrak{S}_{0}$.

(d) If $1 \subset \mathfrak{A} \triangleleft \mathfrak{S}_{2}$, then either $N(\Re) \subseteq \mathfrak{M}$ or $\mathfrak{S}_{2}$ is a $S_{2}$-subgroup of $N(\Omega)$.

Then $\mathfrak{S}_{0} \subseteq \mathfrak{M}$.

Proof. Suppose false. Let $\mathfrak{S}$ be a subgroup of $\mathfrak{S}_{0}$ which contains $\mathfrak{S}_{2}$ and is minimal subject to $\mathfrak{S} \nsubseteq \mathfrak{M}$. By $(a), O_{2},(\mathfrak{S})=1$. By minimality of $\subseteq$, we have $\subseteq=\mathfrak{S}_{2} \mathfrak{Q}$ for some $q$-group $\mathfrak{Q}$, and $\mathfrak{M} \cap \mathfrak{Q}=\boldsymbol{D}(\mathfrak{Q})$, while if $\mathfrak{S}=\boldsymbol{O}_{2}(\mathfrak{S})$, then $\mathfrak{S} \mathfrak{S} / \mathfrak{S C}_{\mathcal{C}}(\mathfrak{\Omega})$ is a chief factor of $\mathfrak{S}$. Suppose $G \in \mathbb{S}$ and $\mathfrak{F}^{G} \cap \mathfrak{S}_{2}$ is non cyclic. We will show that $\mathfrak{F}^{G} \cap \mathfrak{S}_{2} \subseteq \mathfrak{S}_{\mathrm{s}}$. Suppose false. Then $G \notin \mathfrak{M}$, since $\mathfrak{F} \subseteq \boldsymbol{O}_{2}(\mathfrak{S})$. Let $\mathfrak{F}^{*}=\mathfrak{F}^{G} \cap \mathfrak{S}_{2}$.

Let $\Omega_{1}$ be a subgroup of $\cong$ minimal subject to (a) $\Omega_{1} \nsubseteq \boldsymbol{D}(\Omega)$, (b) $\mathfrak{S}_{\mathfrak{2}} \mathfrak{Q}_{1}$ admits $\mathfrak{F}^{*}$, (c) $\left[\mathfrak{\Omega}_{1}, \mathfrak{F}^{*}\right] \nsubseteq \mathfrak{S}_{\text {. }}$ Then $\mathfrak{\Omega}_{1}$ is cyclic. Let $\mathfrak{S}_{1}=$ $\mathfrak{S}_{\mathfrak{S}} \mathfrak{O}_{1} \mathfrak{F}^{*}, \mathfrak{H}_{1}=\boldsymbol{O}_{2}\left(\mathfrak{S}_{1}\right)$, and let $\mathfrak{F}_{1}^{*}=\mathfrak{F}^{*} \cap \mathfrak{S}_{1}$. Thus, $\mathfrak{F}_{1}^{*}$ is of index 2 in $\mathfrak{F}^{*}$, so is not 1 . Since $\mathfrak{\Omega}_{1} \nsubseteq \mathfrak{M}, \mathfrak{\Omega}_{1}$ does not centralize $Z\left(\mathfrak{S}_{1}\right)$. Choose 
$F^{*} \in \mathfrak{F}^{*}-\mathfrak{F}_{1}^{*}$. We assume without loss of generality that $F^{*}$ inverts a generator $Q$ of $\mathfrak{\Omega}_{1}$. Now $Z\left(\mathfrak{S}_{1}\right) \subseteq C\left(\mathfrak{F}_{1}^{*}\right) \subseteq \mathfrak{M}^{a}$. Since $\mathfrak{\Omega}_{1}$ does not centralize $\boldsymbol{Z}\left(\mathfrak{S}_{1}\right)$, neither does $F^{*}$, so $\left[\boldsymbol{Z}\left(\mathfrak{S}_{1}\right), F^{*}\right] \neq 1$. Hence, $\boldsymbol{Z}\left(\mathfrak{S}_{1}\right) \cap$ $\mathfrak{F}^{G} \neq 1$, so $\mathfrak{S}_{1} \subseteq \mathfrak{M}^{G}$.

Let $\Re=\left[\mathfrak{S}_{1}, \mathfrak{\Omega}_{1}\right], \quad \mathfrak{\Re}_{0}=\mathfrak{S}_{1} \cap \boldsymbol{C}\left(\mathfrak{\Omega}_{1}\right), \Re_{1}=\left[\Re, F^{*}\right]$. Then $\mathfrak{S}_{1}=\Re_{\Omega_{0}}$ and $\Re_{0} \cap \mathfrak{F}^{G}=1$, so that $F^{*}$ centralizes $\Omega_{0}$. Also, $\Re=\Re_{1} \times \Re_{1}^{Q}$ is elementary. On the other hand, $\mathfrak{F} \subseteq \mathfrak{F}_{\mathfrak{C}} \subseteq \mathfrak{F}_{1}$, so $\mathfrak{F} \subseteq \mathfrak{M}^{G}$. By Lemma 13.30, $\left[\mathfrak{F}, \mathfrak{F}^{G}\right]=1$. Hence, $\mathfrak{F} \subseteq \Re_{0} \Re_{1}$. Since $|\mathfrak{F}|=\left|\mathfrak{F}^{G}\right|>\left|\Re_{1}\right|$, it follows that $\mathfrak{F} \cap \Re_{0} \neq 1$. Since $\mathfrak{\Omega}_{1}$ centralizes $\Re_{0}$, we get $\mathfrak{\Omega}_{1} \subseteq \mathfrak{M}$, contrary to our contruction.

By the previous argument, we get $V\left(\operatorname{ccl}_{\circlearrowleft}\left(\widetilde{F}_{0}\right) ; \mathfrak{S}_{2}\right) \triangleleft \subseteq$ for all non cyclic subgroups $\mathfrak{F}_{0}$ of $\mathfrak{F}$. By (d), we get that $\mathfrak{S}_{2}=\mathfrak{T}$. Hence, both $\mathfrak{W}$ and $\mathfrak{W}^{*}$ are normal in $\subseteq$. Since $\mathfrak{X}=\Omega_{1}\left(\mathbb{Z}\left(\mathfrak{W}^{*}\right)\right)$, we get $\mathfrak{X} \triangleleft \mathfrak{S}$. By Lemma 13.34, we get $\subseteq \subseteq \mathfrak{M}$, against our construction.

Lemma 13.38. If $G \in \mathbb{S}$, then one of the following holds:

(a) $\mathfrak{F}^{G} \cap \mathfrak{M}=1$.

(b) $\mathfrak{F}^{G} \cap \mathfrak{M}$ is of index 2 in $\mathfrak{F}^{G}$ and $\mathfrak{F}^{G} \cap \mathfrak{M}$ centralizes a hyperplane of $\mathfrak{F}$.

(c) $\mathfrak{F}^{a} \subseteq \mathfrak{M}$.

Proof. Suppose $1 \subset \mathfrak{F}^{G} \cap \mathfrak{M} \subset \mathfrak{F}^{G}$. Set $\mathfrak{F}^{*}=\mathfrak{F}^{G}$, $\mathfrak{F}_{1}^{*}=\mathfrak{F}^{G} \cap \mathfrak{M}, \mathfrak{F}_{1}=$

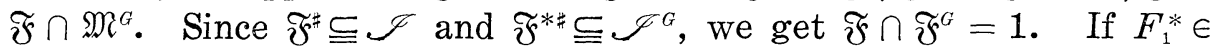
$\mathfrak{F}_{1}^{* \#}$, then $\left[\mathfrak{F}_{1}^{*}, C_{\mathfrak{F}}\left(F_{1}^{*}\right)\right] \leqq \mathfrak{F}_{\mathfrak{F}^{\prime}} \cap \mathfrak{F}^{*}=1$, while if $F_{1} \in \mathfrak{F}_{1}^{*}$, then $\left[\mathfrak{F}_{1}, C_{\mathfrak{F}^{*}}\left(F_{1}\right)\right] \subseteq$ $\mathfrak{F} \cap \mathfrak{F}^{*}=1$. Since $\mathfrak{F}^{*} \nsubseteq \mathfrak{M}$, so also $\mathfrak{F} \nsubseteq \mathfrak{M}^{G}$, by Lemma 13.30. Let $\mathfrak{\mho}_{2}=N_{\mathfrak{F}}\left(\mathfrak{\mho}_{1} \mathfrak{\mho}_{1}^{*}\right), \mathfrak{\mho}_{2}^{*}=N_{\mathfrak{F}^{*}}\left(\mathfrak{F}_{1}^{*} \mathfrak{\mho}_{1}\right)$, so that $\mathfrak{\mho}_{2} \supset \mathfrak{\mho}_{1}, \mathfrak{\mho}_{2}^{*} \supset \mathfrak{F}_{1}^{*}$. Choose $F_{2} \in \mathfrak{F}_{2}-\widetilde{F}_{1}$. Then $\left[F_{2}, \mathfrak{F}_{2}^{*}\right] \leqq \mathfrak{F}_{1}$ and $C\left(F_{2}\right) \cap \mathfrak{F}_{1}^{*}=1$. Hence $\left|\mathfrak{F}_{1}\right| \geqq\left|\mathfrak{F}_{1}^{*}\right|$. By symmetry, we get $\left|\mathfrak{F}_{1}^{*}\right| \geqq\left|\mathfrak{F}_{1}\right|$, so $\left|\mathfrak{\mho}_{1}\right|=\left|\mathfrak{F}_{1}^{*}\right|$. Let $\mathfrak{S}=\mathfrak{F}_{1} \mathfrak{\mho}_{1}^{*}=$ $\mathfrak{F}_{1} \times \mathfrak{F}_{1}^{*}$. Let $\mathscr{C}, \mathscr{C}^{*}$ be the chains $\mathfrak{\mathcal { C }} \supset \mathfrak{F}_{1} \supset 1, \mathfrak{S} \supset \mathfrak{F}_{1}^{*} \supset 1$, respectively. Then $\mathfrak{F}_{2} / \mathfrak{F}_{1}$ maps isomorphically into $\boldsymbol{A}_{\mathscr{G}}(\mathscr{C})$ and $\mathfrak{F}_{2}^{*} / \mathfrak{F}_{1}^{*}$ maps isomorphically into $\boldsymbol{A}_{\Theta}\left(\mathscr{C}^{*}\right)$, and if $F_{2} \in \mathfrak{F}_{2}-\mathfrak{F}_{1}$, then the map $\varphi\left(F_{2}\right): \mathfrak{F}_{1}^{*} \rightarrow \mathfrak{F}_{1}$, given by $F_{1}^{*} \mapsto\left[F_{2}, F_{1}^{*}\right]$ is an isomorphism of $\widetilde{F}_{1}^{*}$ onto $\widetilde{F}_{1}$. Since $N(\mathfrak{F})$ is solvable, it follows that $\left|\mathfrak{F}_{2}: \mathfrak{F}_{1}\right|=\left|\mathfrak{F}_{2}^{*}: \mathfrak{F}_{1}^{*}\right|=2$. If $\mathfrak{F}_{2}=\mathfrak{F}$, we are done, so suppose $\mathfrak{F}_{2} \subset \mathfrak{F}$. Let $\mathfrak{F}_{2}=\mathfrak{F}_{1} \times\left\langle F_{2}\right\rangle$. Choose $F$ in $\mathfrak{F}_{-}-\mathfrak{F}_{2}$ with $\left[F, \mathfrak{F}_{1}^{*}\right] \subseteq \mathfrak{F}_{2}$. Since $F$ does not normalize $\mathfrak{F}_{1} \mathfrak{F}_{1}^{*}$, we can choose $F_{1}^{*} \in \mathfrak{F}_{1}^{*}$ such that $\left[F, F_{1}^{*}\right] \notin \mathfrak{F}_{1}$. Hence, $\left[F, F_{1}^{*}\right]=F_{2} F_{1}$ with $F_{1} \in \mathfrak{F}_{1}$. Let $\mathfrak{F}_{1}^{* *}$ be the largest subgroup of $\mathfrak{F}_{1}^{*}$ such that $\left[F, \mathfrak{F}_{1}^{* *}\right] \subseteq \mathfrak{F}_{1}$. Hence, $\mathfrak{F}_{1}^{*}=\mathfrak{F}_{1}^{* *} \times\left\langle F_{1}^{*}\right\rangle$. Since $\left|\mathfrak{F}_{1}^{*}\right| \geqq 4$, we have $\mathfrak{F}_{1}^{* *} \neq 1$. Choose $F_{1}^{* *} \in$ $\mathfrak{\mho}_{1}^{* * *}$. By the Jacobi identity, $\left[F, F_{1}^{*}, F_{1}^{* *}\right]\left[F_{1}^{* *}, F, F_{1}^{*}\right]=1$, since $\left[F_{1}^{*}, F_{1}^{* *}\right]=1$. This is not the case, since $\left[F, F_{1}^{*}, F_{1}^{* *}\right]=\left[F_{2}, F_{1}^{* *}\right] \neq 1$, while $\left[F_{1}^{* *}, F\right] \in \widetilde{F}_{1}$, so that $\left[F_{1}^{* *}, F, F_{1}^{*}\right]=1$. The proof is complete.

Theorem 13.3. Hypothesis 13.2 is not satisfied. 
Proof. Suppose false. If $\mathfrak{T} \subseteq \mathfrak{S}_{0} \in \mathscr{S} \mathscr{O} \mathscr{L}(\mathbb{S})$, then by Lemma 13.36, $\mathfrak{S}_{0}$ satisfies the hypotheses of Lemma 13.37, so $\mathfrak{S}_{0} \subseteq \mathfrak{M}$, that is, $\mathfrak{I} \in \mathscr{L}^{*}(\mathbb{S})$. Thus, we may apply Lemma 13.2. Let $\mathfrak{S}$ be the group given in Lemma 13.2. We may then choose $M$ in $\mathfrak{M}$ such that $\mathfrak{S C}^{M} \cap \mathfrak{I}$ is a $S_{2}$-subgroup of $\mathfrak{S}^{M}$. Thus, we may assume at the outset that $\mathfrak{S} \cap \mathfrak{I}=\mathfrak{S}_{2}$ is a $S_{2}$-subgroup of $\mathfrak{S}$. Let $I$ be an involution of $\mathfrak{M}$ such that $C_{\tilde{\mathfrak{x}}_{2}}(I)=\mathfrak{I}_{2}$ is a $S_{2}$-subgroup of $C_{\mathfrak{m}}(I)$.

Let $\mathfrak{S} \subseteq S \in \mathscr{C} \mathscr{S}(\mathbb{S})$. By Lemma 13.2, $\mathfrak{S}$ contains an element $\mathfrak{U}$ of $\mathscr{U}(2)$, so $O_{2}(\mathfrak{S}) \neq 1$. By Lemma 13.1 , we get $O_{2^{\prime}}(\mathfrak{S})=1$, since $\mathfrak{S}=\boldsymbol{N}\left(\boldsymbol{O}_{2}(\mathfrak{S})\right)$. By Lemma 13.20, $\mathfrak{S}$ has no elementary subgroup of order $p^{3}$ for any odd prime $p$.

By Lemma $13.2, \mathfrak{S}=\mathfrak{S}_{2} \mathfrak{Q}$, where $\mathfrak{Q}$ is a 3 -group. By the preceding paragraph, $\mathscr{S} \mathscr{C} \mathscr{N}_{3}(\mathfrak{D})=\varnothing$. We assume without loss of generality that if $\mathfrak{S}_{2} \subseteq \mathfrak{S}_{0} \subset \mathfrak{S}$, then $\mathfrak{S}_{0} \subseteq \mathfrak{M}$. Hence, $\mathfrak{M} \cap \mathfrak{Q}=\boldsymbol{D}(\mathfrak{D})$ and if $\boldsymbol{O}_{2}(\mathfrak{S})=\Re$, then $\mathscr{R} \Omega / \Re D(\Omega)$ is a chief factor of $\mathscr{S}$.

Let $\mathfrak{F}_{0}=\mathfrak{F} \cap \mathfrak{S}_{,}, \mathfrak{\mho}_{1}=\mathfrak{F} \cap \Re=\mathfrak{F}_{0} \cap \Re$. By Lemma 13.37, $\mathfrak{\mho}_{1} \subset \mathfrak{F}$. We argue that $\mathfrak{F}_{1} \subset \mathfrak{F}_{0}$. For suppose $\mathfrak{F}_{1}=\mathfrak{F}_{0}$. Let $\mathfrak{F}_{2} / \mathfrak{F}_{1}$ be a chief factor of $\mathfrak{F}_{\mathfrak{C}_{2}}$, with $\mathfrak{\mho}_{2} \subseteq \mathfrak{F}$. Then $\left[\Re, \mathfrak{F}_{2}\right] \subseteq \widetilde{\mho}_{1}=\mathfrak{F}_{0} \subseteq \Re$, so that $\widetilde{\mho}_{2} \subseteq$ $N(\Re)$. Since $\mathfrak{S}_{2}$ is a $S_{2}$-subgroup of $N(\Re)$, we get $\mathfrak{F}_{2} \subseteq \mathfrak{S}_{2} \cap \mathfrak{F}=\mathfrak{F}_{1}=\mathfrak{F}_{0}$, against $\mathfrak{F}_{1}=\mathfrak{F}_{0} \subset \mathfrak{F}_{2}$. Hence, $\mathfrak{F}_{1} \subset \mathfrak{F}_{0} \subseteq \mathfrak{F}$.

Since $\boldsymbol{C}_{\mathfrak{F}}(I)$ is non cyclic, and since $\mathfrak{S}_{2}$ contains a $S_{2}$-subgroup of $C_{\mathbb{R}}(I)$, it follows that $\widetilde{\mho}_{0}$ is non cyclic.

Now $\mathfrak{F}_{0} \Re / \Re \triangleleft \mathfrak{S}_{2} / \Re$, so that $\Re\left[\Omega, \mathfrak{F}_{0}\right] D(\Omega) \triangleleft \mathfrak{S}_{2}$. Since $\Re \mathfrak{\Omega} / \Re D(\mathfrak{Q})$ is a chief factor of $\mathfrak{K}$, we get $\Re \Omega=\Re\left[\Omega, \mathfrak{\mho}_{0}\right] D(\Omega)$, so that $\Re \Omega=$

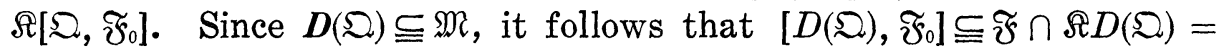
$\mathfrak{F} \cap \Re=\mathfrak{F}_{1}$. By Lemma 0.8.7, $D(\Omega) \subseteq Z(\Omega)$. This implies that $\Omega$ is of exponent 3 , and either $|\mathfrak{Q}| \leqq 3^{2}$, or $\mathfrak{Q}$ is non abelian of order $3^{3}$. If $\mathfrak{Q}$ is non abelian, then each element of $\mathfrak{F}_{0}$ induces an automorphism of $\Re \Omega / \Re D(\Omega)$ of determinant 1 , since $\mathfrak{F}_{0}$ centralizes $\Re D(\Omega) / \Omega$. In this case, we get $\left|\mho_{0}: ⿱_{1}\right|=2$. The same equality clearly holds if $\left|\mathfrak{D}_{1}\right|=3$. Suppose $|\mathfrak{Q}|=3^{2}$. Then $\mathfrak{\mho}_{0}$ contains an element $F_{0}$ such that $F_{0}$ inverts $\Re \mathfrak{\Omega} / \Omega$, and we assume without loss of generality that $F_{0}$ inverts $\Omega$. If $Q \in \mathfrak{\Omega}$, then $C_{\Omega}(Q)$ admits $F_{0}$, so $\left[C_{\Omega}(Q), F_{0}\right] \subseteq \widetilde{\mho} \cap C_{\Omega}(Q)=1$. Hence, $F_{0}$ centralizes $\left\langle C_{\Omega}(Q) \mid Q \in \Omega^{\sharp}\right\rangle=\Omega$, against $F_{0} \notin \Re$. We conclude that $|\mathfrak{Q}|=3$ or $3^{3}$ and that $\left|\mathfrak{\mho}_{0}: \mathfrak{\mho}_{1}\right|=2$.

Choose $F_{0} \in \mathfrak{F}_{0}-\mathfrak{F}_{1}$. Then $\mathfrak{F}_{0}$ inverts a subgroup $\mathfrak{Q}_{1}$ of $\mathfrak{R}$ of

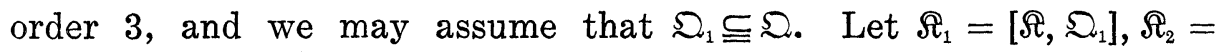
$\left[\Omega_{1}, F_{0}\right], \Re_{0}=C_{\Re}\left(\Omega_{1}\right)$. Thus, $\Re_{2} \subseteq \Im$, so that $\Re_{1}=\Re_{2} \times \Re_{2}^{Q}$, where $Q$ is a generator for $\mathfrak{D}_{1}$. Since $\Re_{0} \cap \mathfrak{F}=1$, we get $\mathfrak{\mho}_{1}=\Re_{2} \neq 1$. Suppose $\left|\Re_{1}\right| \geqq 2^{4}$. In this case, it follows that $J\left(\mathfrak{S}_{2}\right) \cong \Re$, so that $J\left(\left(\mathfrak{S}_{2}\right) \triangleleft \mathfrak{S}\right.$. This is not the case, since $\left.\mid N\left(J\left(\mathfrak{C}_{2}\right)\right)\right)\left.\right|_{2}>\left|\mathfrak{S}_{2}\right|$. Hence, $\left|\Re_{1}\right|=2^{2},\left|\mho_{0}\right|=$ $2^{2},\left|\mho_{1}\right|=2$. This in turn implies that $\left|\Omega_{1}\right|=3, \Omega=\Omega_{1}$, and so $\mathfrak{S}=\Re_{2} \times \Re_{1} \mathfrak{Q}_{1}\left\langle F_{0}\right\rangle$, with $\Re_{1} \mathfrak{\Omega}_{1}\left\langle F_{0}\right\rangle \cong \Sigma_{4}$.

Since no non identity characteristic subgroup of $\mathfrak{K}_{2}$ is contained 
in $\Re_{0}$, it follows that $\boldsymbol{D}\left(\Re_{0}\right)$ is elementary and central in $\Re_{0}$. Thus, $\boldsymbol{D}\left(\mathfrak{S}_{2}\right)=\boldsymbol{D}\left(\Re_{0}\right) \times \mathfrak{F}_{1}$. Choose $X \in \boldsymbol{N}_{\Re}\left(\mathfrak{S}_{2}\right)-\mathfrak{S}_{2}$. Then $\boldsymbol{Z}\left(\Re_{0}\right) \cap \boldsymbol{C}(X)=1$, since $\mathfrak{S}_{2}$ is a $S_{2}$-subgroup of $\boldsymbol{N}(\mathfrak{Y})$ for every non identity normal subgroup $\mathfrak{Y}$ of $\mathfrak{K}$. Hence, $\left|\boldsymbol{D}\left(\Re_{0}\right)\right| \leqq 2,\left|\boldsymbol{Z}\left(\Re_{0}\right)\right| \leqq 2$. Now $X$ normalizes $\mathfrak{S}_{2} \cap C\left(\widetilde{F}_{0}\right)=\Re_{0} \times \widetilde{F}_{0}$. Hence, $\mathfrak{\Re}_{0}$ is elementary, so $\left|\mathfrak{\Re}_{0}\right| \leqq 2$. If $\Re_{0}=1$, then $\mathfrak{S}_{2}$ is dihedral of order $2^{3}$, against the presence of $I$. So suppose $\left|\Re_{0}\right|=2$. In this case, $\mathfrak{F}_{2}$ has exactly 2 elementary subgroups of order $2^{3}$, namely, $\Re_{0} \times \mathfrak{F}_{0}$ and $\Omega_{\text {. Since }} X$ normalizes $\Re_{0} \times \mathfrak{F}_{0}, X$ also normalizes $\Re$. This contradiction completes the proof.

REMARK. Theorem 13.3 is one of the watersheds of this work. While there is still a great deal to be done, we are now reduced to examining a succession of explicit groups. The "generic case" is completed.

HYPOTHESIS 13.3.

(a) $\subseteq \in \mathscr{L} \mathscr{S}$ (S).

(b) $O_{2^{\prime}}(\mathfrak{S})=1$.

(c) $m(\mathfrak{X}) \leqq 2$ for every normal abelian subgroup $\mathfrak{A}$ of $\mathfrak{S}$.

(d) $\subseteq$ contains a non cyclic normal abelian subgroup $\mathfrak{B}$ such that $C(B) \subseteq \subseteq$ for all $B$ in $\mathfrak{B}^{*}$.

Lemmas 13.39 through 13.53 are proved under Hypothesis 13.3. We use the following notation: $\mathfrak{B}$ is a normal abelian subgroup of (5) which contains $\mathfrak{B}$ and is maximal with this restriction, and $\mathfrak{B}_{0}=$ $\Omega_{1}(\mathfrak{B})$. By $(\mathrm{c})$ and $(\mathrm{d})$, we get $m(\mathfrak{B})=m(\mathfrak{B})=2$, so that $\Omega_{1}(\mathfrak{B})=\mathfrak{B}_{0}$

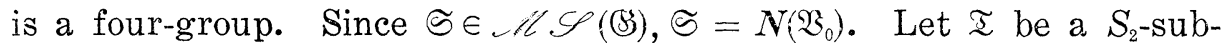
group of $\subseteq$.

LEMma 13.39. T is a $S_{2}$-subgroup of (S) and $N(\mathfrak{T}) \subseteq \subseteq$.

Proof. Let $3=\Omega_{1}(\boldsymbol{Z}(\mathfrak{I}))$. Since $\boldsymbol{O}_{2^{\prime}}(\mathfrak{S})=1$, we get $3 \subseteq \boldsymbol{O}_{2}(\subseteq)$. Thus, $\left\langle\mathcal{B}, \mathfrak{B}_{0}\right\rangle \subseteq\left\langle\mathfrak{B}, Z\left(\boldsymbol{O}_{2}(\subseteq)\right)\right\rangle$, and the group on the right is a normal abelian 2-subgroup of $\subseteq$. Hence, $3 \subseteq \mathfrak{B}_{0}$ by Hypothesis $13.3(\mathrm{c})$. If $3=\mathfrak{B}_{0}$, then we get $\subseteq=N(3)$, and we are done. Suppose $3 \subset \mathfrak{B}_{0}$, so that $|3|=2$. Hence, $N(3)=C(3) \subseteq \subseteq$, by (d), and we are done.

Lemma 13.40. $\mathfrak{B}$ is a T.I. set in (3).

Proof. Suppose false. Then there is $G$ in $(8)-\subseteq$ such that $\mathfrak{B} \cap \mathfrak{B}^{G} \neq 1$. Let $V$ be an involution of $\mathfrak{B} \cap \mathfrak{B}^{G}$. By Hypothesis 13.3(d), $C(V) \subseteq \Im \cap \Im^{G}$.

Case 1. $C(V)$ contains an $S_{2}$-subgroup $\mathfrak{I}^{*}$ of 6 . Since $\mathfrak{I}^{*} \subseteq \subseteq \cap \mathfrak{S}^{*}$, we get $\mathfrak{I}^{* G^{-1}} \subseteq \subseteq$. Hence, $\mathfrak{I}^{* G^{-1}}=\mathfrak{I}^{* S}$ for some $S$ in $\mathfrak{S}$. Hence, 
$S G \in N\left(\mathfrak{I}^{*}\right)$. By Lemma 13.39, we get $N\left(\mathfrak{T}^{*}\right) \subseteq \subseteq$. Hence, $G \in S^{-1} \mathfrak{S}=\Subset$, against our choice of $G$.

Case 2. $C(V)$ does not contain a $S_{2}$-subgroup of (\$).

Let $\mathfrak{I}_{0}$ be a $S_{2}$-subgroup of $C(V)$, so that $\mathfrak{I}_{0} \subseteq \mathfrak{S}$. Let $\mathfrak{I}^{*}$ be a $S_{2}$-subgroup of $\mathfrak{S}$ which contains $\mathfrak{I}_{0}$. Since $V \in \mathfrak{B}_{0} \triangleleft \mathfrak{T}^{*}$, it follows that $\left|\mathfrak{I}^{*}: \mathfrak{I}_{0}\right|=2$, and that $\mathfrak{I}_{0}=C_{\mathfrak{R}^{*}}(V)=C_{\mathfrak{R}^{*}}\left(\mathfrak{B}_{0}\right)$. Since $V$ is not contained in the center of any $S_{2}$-subgroup of $\mathbb{B}$, $\mathfrak{S}$ does not permute $\mathfrak{S}_{0}^{\#}$ transitively. Hence, $\mathfrak{I}^{*} / \mathfrak{I}_{0}$ maps onto $\boldsymbol{A}_{\mathscr{\Theta}}\left(\mathfrak{B}_{0}\right)$. Hence, $\boldsymbol{C}_{\Theta}(V)=$ $\boldsymbol{C}_{\odot}(V)=\boldsymbol{C}_{\Phi^{G}}(V) \triangleleft\left\langle\mathfrak{S}, \mathfrak{S}^{G}\right\rangle$, against $\subseteq \in \mathscr{C} \mathscr{S}(\mathbb{S})$. The proof is complete.

Lemma 13.41. Suppose $G \in \mathfrak{B S}$ and $\mathfrak{B}^{G} \subseteq \mathfrak{S}$. Then $\left[\mathfrak{B}, \mathfrak{B}^{G}\right]=1$.

Proof. Let $\tilde{\mathfrak{B}}=\mathfrak{B} \cap \boldsymbol{C}\left(\mathfrak{B}^{C}\right)$, and suppose by way of contradiction that $\tilde{\mathfrak{B}} \subset \mathfrak{B}$. Let $\mathfrak{B}_{1} / \tilde{\mathfrak{B}}$ be a chief factor of $\mathfrak{B} \cdot \mathfrak{B}^{G}$ with $\mathfrak{B}_{1} \subseteq \mathfrak{B}$. Since $|\tilde{\mathfrak{B}}|<\left|\mathfrak{B}^{G}\right|$, and since $\left[\mathfrak{B}_{1}, \mathfrak{B}^{G}\right] \subseteq \tilde{\mathfrak{B}}$, it follows that there is $V$ in $\mathfrak{B}^{G^{\sharp}}$ such that $\mathfrak{B}_{1} \subseteq C(V)$. By Hypothesis $13.3(\mathrm{~d}), \mathfrak{B}_{1} \subseteq \mathfrak{S}^{G}$, so that $\left[\mathfrak{B}_{1}, \mathfrak{B}^{\Re}\right] \subseteq$ $\mathfrak{B} \cap \mathfrak{B}^{G}$. By Lemma $13.40, \mathfrak{B} \cap \mathfrak{B}^{G}=1$, so $\mathfrak{B}_{1} \subseteq \tilde{\mathfrak{B}}$, against our construction.

Lemma 13.42. Suppose $G \in \mathbb{C S}$ and $\left|\mathfrak{B}^{a}: \mathfrak{B}^{\alpha} \cap \mathfrak{S}\right| \leqq 2$. Then one of the following holds:

(a) $\mathfrak{B}^{G} \subseteq \mathfrak{S}$.

(b) $\mathfrak{B}$ is a four-group.

(c) $\mathfrak{B}$ is of type $(2,4)$ and $\mathfrak{B}^{G} \cap \mathfrak{S}$ is a four-group.

Proof. Suppose $\left|\mathfrak{B}^{G}: \mathfrak{B}^{G} \cap \mathfrak{S}\right|=2$. Let $\mathfrak{B}$ be of type $\left(2^{a}, 2^{b}\right)$ with $1 \leqq a \leqq b$. We must show that $a=1, b \leqq 2$, and that if $b=2$, then $\mathfrak{B}^{G} \cap \subseteq$ is a four-group.

Let $\mathfrak{B}^{*}=\mathfrak{B}^{G}, \mathfrak{B}_{1}^{*}=\mathfrak{B}^{*} \cap \mathfrak{S}$. Let $\mathfrak{B}_{1}=\mathfrak{B} \cap \boldsymbol{C}\left(\mathfrak{B}_{1}^{*}\right)$. If $\mathfrak{B}_{1}=\mathfrak{B}$, then $\mathfrak{B} \cong \mathfrak{S}^{a}$, so by Lemma 13.41 applied to $\mathfrak{S}^{G}$, we get $\left[\mathfrak{B}^{G}, \mathfrak{B}\right]=1$. Thus, $\mathfrak{B}^{G} \subseteq \mathfrak{S}$, against our assumption. Hence, $\mathfrak{B}_{1} \subset \mathfrak{B}$. Let $\mathfrak{B}_{2} / \mathfrak{B}_{1}$ be a chief factor of $\mathfrak{B}_{1}^{*}$ with $\mathfrak{B}_{2} \subseteq \mathfrak{B}$. Choose $V_{2} \in \mathfrak{B}_{2}-\mathfrak{B}_{1}$. If $\mathfrak{B}_{1}^{*} \cap C\left(V_{2}\right) \neq 1$, then $V_{2} \in \mathfrak{S}^{G}$, so that $\left[V_{2}, \mathfrak{B}_{1}^{*}\right] \subseteq \mathfrak{B}^{G} \cap \mathfrak{B}=1$. This is not the case, by definition of $\mathfrak{B}_{1}$ so $\mathfrak{B}_{1}^{*} \cap \boldsymbol{C}\left(V_{2}\right)=1$. Since $\left[V_{2}, \mathfrak{B}_{1}^{*}\right] \subseteq \mathfrak{B}_{1}$, we get $\left|\mathfrak{B}_{1}\right| \geqq$ $\left|\mathfrak{B}_{1}^{*}\right|=|\mathfrak{B}| / 2$. Hence, $\mathfrak{B}_{2}=\mathfrak{B}$, and $\left|\mathfrak{B}_{:} \mathfrak{B}_{1}\right|=2$.

Let $\mathfrak{S}=\mathfrak{B}_{1} \mathfrak{B}_{1}^{*}=\mathfrak{B}_{1} \times \mathfrak{B}_{1}^{*}$. Since $\mathfrak{B} \nsubseteq \mathfrak{S}^{G}$, we get $\mathfrak{B}_{1}=\mathfrak{B} \cap \mathfrak{S}^{G}$. For each $V$ in $\mathfrak{B}-\mathfrak{B}_{1}$, we see that $V$ normalizes $\mathfrak{B}_{1}^{*} \cap \mathfrak{B}_{1}^{* V}$, since $V^{2} \in \mathfrak{B}_{1} \subseteq C\left(\mathfrak{B}_{1}^{*}\right)$. If $\mathfrak{B}_{1}^{*} \cap \mathfrak{B}_{1}^{* V} \neq 1$, choose $V^{*}$ an involution of $\mathfrak{B}_{1}^{*} \cap$ $\mathfrak{B}_{1}^{*^{V}} \cap \boldsymbol{C}(V)$, and conclude that $V \in C\left(V^{*}\right) \subseteq \varsigma^{G}$, against $\varsigma^{G} \cap \mathfrak{B}=\mathfrak{B}_{1}$. So $\mathfrak{B}_{1}^{*} \cap \mathfrak{B}_{1}^{*^{V}}=1$. Since $\left|\mathfrak{B}_{1}^{* V}\right|=\left|\mathfrak{B}_{1}^{*}\right|=\left|\mathfrak{B}_{1}\right|$, it follows that

$$
\mathfrak{S}=\mathfrak{B}_{1}^{*} \times \mathfrak{B}_{1}^{* V} \text {. }
$$


Since $V^{2} \in \mathfrak{B}_{1} \subseteq \mathfrak{S}$, we have

$$
V^{2}=X^{*} \cdot X_{1}^{* V}, \quad X^{*}, X_{1}^{*} \in \mathfrak{B}_{1}^{*} \cdot
$$

Hence, $V^{2}=V^{2 V}=X^{*} \cdot X_{1}^{*^{V}}=X^{* V} \cdot X_{1}^{*}$, so that

$$
X^{*} \cdot X_{1}^{*^{-1}}=\left(X^{*} \cdot X_{1}^{*^{-1}}\right)^{V} \in \mathfrak{B}_{1}^{*} \cap \mathfrak{B}_{1}^{*^{V}}=1,
$$

whence

$$
X^{*}=X_{1}^{*}, \quad V^{2}=X^{*} \cdot X^{* V}=X^{*^{2}}\left[X^{*}, V\right] .
$$

Since $V^{2}$ and $\left[X^{*}, V\right]$ are in $V$, we get

$$
X^{* 2} \in V \cap V_{1}^{*}=1 \text {. }
$$

Hence, $V^{4}=1$, and $V=X^{*-1} V^{-1} X^{*}$. Since $\mathfrak{B}_{1}$ is of index 2 in $\mathfrak{B}, \mathfrak{B}$ is generated by $\mathfrak{B}-\mathfrak{B}_{1}$, and so $b \leqq 2$. If $b=1$, then $a=1$ and we are done. We may assume that $b=2$.

Case 1. $a=1$.

Here we must show that $\mathfrak{B}_{1}^{*}$ is a four-group. Since $\left|\mathfrak{B}_{1}\right|=4$, and $\mathfrak{B}_{1} \subseteq \mathfrak{B}^{G}$, it follows that some $V$ of $\mathfrak{B}_{1}^{*}$ centralizes $\Omega_{1}\left(\mathfrak{B}^{G}\right)$. Hence, $\Omega_{1}\left(\mathfrak{B}^{G}\right) \subseteq C(V) \subseteq \mathfrak{S}$. Since $\subseteq \cap \mathfrak{B}^{G}=\mathfrak{B}_{1}^{*}$, we get $\mathfrak{B}_{1}^{*}=\Omega_{1}\left(\mathfrak{B}^{G}\right)$.

Case 2. $a=2$.

In this case, it follows that for suitable $V, W \in \mathfrak{B}, \mathfrak{B}=\langle V\rangle \times\langle W\rangle$, $\mathfrak{B}_{1}=\left\langle V^{2}\right\rangle \times\langle W\rangle$. For each $X$ in $\mathfrak{B}_{1}^{*}$, we get $V^{x}=V \cdot V^{2 x} W^{y}$, for suitable $x, y$. Since $X$ centralizes $V^{2}$, we get $y=2 z$ for suitable integral z. Thus, $\mathfrak{B}_{1}^{*}$ stabilizes $\mathfrak{B} \supset \mathfrak{B}_{1} \supset 1$ and also stabilizes $\mathfrak{Y} \supset \mathfrak{B}_{0} \supset 1$. As the intersection of the two stability groups is of order $4, \mathfrak{B}_{1}^{*}$ does not act faithfully on $\mathfrak{B}$. This contradiction completes the proof.

Lemma 13.43. Suppose $\mathfrak{T} \subseteq \mathfrak{R} \in \mathscr{S} \mathscr{O} \mathscr{L}(\mathbb{S})$ and $\mathfrak{R} \nsubseteq \mathbb{S}$. Then $\mathfrak{N}$ contains no elementary subgroup of order $p^{3}$ for any odd prime $p$.

Proof. Suppose $\mathbb{F}$ is an elementary subgroup of $\mathfrak{R}$ of order $p^{3}, p$ an odd prime. Since $2 \in \pi_{4}$, we get $\boldsymbol{O}_{2^{\prime}}(\mathfrak{R})=1$, so $\boldsymbol{O}_{2}(\mathfrak{R}) \neq 1$. Hence, $U(\Im ; 2)$ is not trivial, so $e(p) \geqq 3$. Hence, Hypothesis 13.1 is satisfied.

Let $\mathfrak{R}^{*}=\boldsymbol{M}(\mathfrak{S}) \supseteqq \mathfrak{N}$. Let $3=\Omega_{1}\left(\boldsymbol{R}_{2}\left(\mathfrak{N}^{*}\right)\right)$. By Lemma $13.19,|3| \leqq 4$. Hence, $\{\cap \boldsymbol{C}(\mathbb{Z}) \in \mathscr{A}(p)$. Let $Z$ be an involution of $3 \cap \boldsymbol{Z}(\mathfrak{I})$. Thus, $Z \in \mathfrak{B}_{0}$. By Hypothesis 13.3 (d), we get $\boldsymbol{C}(Z) \subseteq \Im$. Hence, $\mathbb{F} \cap \boldsymbol{C}(Z) \subseteq \subseteq$. Since $\mathfrak{F} \cap C(Z) \in \mathscr{M}^{*}(\mathbb{S})$, we get $\subseteq \subseteq \mathfrak{N}^{*}$. Since $\subseteq \in \mathscr{M} \mathscr{S}(G)$, we have $\mathfrak{S}=\mathfrak{N}^{*} \supseteqq \mathfrak{N}$, against our assumption that $\mathfrak{N} \nsubseteq \mathfrak{S}$. The proof is complete. 
LEMma 13.44. If $\mathfrak{I} \subseteq \mathfrak{R} \in \mathscr{S} \mathscr{O} \mathscr{L}(\mathfrak{S})$, then $\mathfrak{B} \subseteq \boldsymbol{O}_{2}(\mathfrak{R})$.

Proof. Suppose false. Then $\mathfrak{R} \nsubseteq \mathfrak{S}$. We assume without loss of generality that if $\mathfrak{I} \subseteq \mathfrak{N}_{0} \subset \mathfrak{N}$, then $\mathfrak{B} \subseteq \boldsymbol{O}_{2}\left(\mathfrak{N}_{0}\right)$. Hence, $\mathfrak{N}=\mathfrak{I} \Omega$, where $\mathfrak{Q}$ is a $q$-group for some odd prime $q$. By Lemma 13.43,

$$
\mathscr{S} \mathscr{C} \mathscr{N}_{3}(\mathfrak{D})=\varnothing \text {. }
$$

Let $\mathfrak{K}=\boldsymbol{O}_{2}(\mathfrak{R})$. By minimality of $\mathfrak{R}$, we get $\mathfrak{S} \mathfrak{Q} \backslash \mathfrak{R}$, and $\mathfrak{Y}=$ $\mathfrak{S C O} / \mathfrak{C} D(\mathfrak{O})$ is a chief factor of $\mathfrak{R}$. Let $\mathfrak{X}_{0}=\mathfrak{B} \cap \mathfrak{S}$, so that $\mathfrak{X}_{0} \subset \mathfrak{B}$. Let $\mathfrak{X}_{1} / \mathfrak{X}_{0}$ be a chief factor of $\mathfrak{I}$ with $\mathfrak{X}_{1} \subseteq \mathfrak{B}$. Choose $V \in \mathfrak{X}_{1}-\mathfrak{X}_{0}$. Since $\mathfrak{W}$ is a chief factor of $\mathfrak{N}$ on which $\mathfrak{B}$ acts non trivially, and since $\mathfrak{S} V$ is central in $\mathfrak{I} / \mathfrak{S}$, it follows that $[D(\mathfrak{Q}), V] \subseteq O_{2}(\mathfrak{T} D(\mathfrak{Q})) \cap$ $\mathfrak{S} \boldsymbol{D}(\mathfrak{\Omega})=\mathfrak{S}$. Let $\overline{\mathfrak{Q}}=\mathfrak{S} \mathfrak{Q} / \mathfrak{S}$. Thus, $V$ inverts $\overline{\mathfrak{Q}} / \boldsymbol{D}(\overline{\mathfrak{Q}})$ and $V$ centralizes $\boldsymbol{D}(\overline{\mathfrak{\Omega}})$. This implies that $\boldsymbol{D}(\mathfrak{\Omega}) \subseteq Z(\mathfrak{\Omega})$, and that $\mathfrak{Q}$ is of exponent $q$. Thus, $|\mathfrak{Q}| \leqq q^{2}$, or else $\mathscr{D}$ is extra special of order $q^{3}$.

Let $\overline{\mathfrak{C}}=\mathfrak{S} / \boldsymbol{D}(\mathfrak{E})$. We will show that $\mathfrak{B} / \mathfrak{X}_{0}$ is elementary. Suppose false. Let $V_{1} \in \mathfrak{B}$ satisfy $V_{1}^{2} \notin \mathfrak{X}_{0}, V_{1}^{4} \in \mathfrak{X}_{0}$. Since $\left[\mathfrak{T}, V_{1}, V_{1}\right]=1$, the minimal polynomial of $V_{1}$ on $\overline{\mathfrak{F}}$ divides $(x-1)^{2}$, so $\mathfrak{B}_{1}^{2}$ centralizes $\overline{\mathfrak{F}}$. This is impossible, since $V_{1}^{2} \notin \mathfrak{S}$. Hence, $\mathfrak{B} / \mathfrak{X}_{0}$ is elementary.

We next show that $\left|\mathfrak{B}: \mathfrak{X}_{0}\right|=2$. Suppose false. Since $\mathfrak{B}$ is abelian, $m(\mathfrak{B})=2$ and $\mathfrak{B} / \mathfrak{X}_{0}$ is elementary, we may assume that $\mathfrak{B} / \mathfrak{X}_{0}$ is of order 4 .

Let $\overline{\mathfrak{Q}}_{0}$ be a subgroup of $\overline{\mathfrak{Q}}=\mathfrak{S} \mathfrak{S} / \mathfrak{S}$ of order $q$ which admits $\mathfrak{B}$ and is not centralized by $\mathfrak{B}$. Let $\tilde{\mathfrak{B}}=\mathfrak{B} \cap C\left(\widetilde{\mathfrak{N}}_{0}\right)$, so that $|\mathfrak{B}: \tilde{\mathfrak{B}}|=2$. Let $\overline{\mathfrak{Q}}_{0}=\mathfrak{C}_{0} \mathfrak{\Omega}_{0} / \mathfrak{C}$, where $\mathfrak{\Omega}_{0} \subseteq \mathfrak{D}$, and let $Q$ be a generator for $\mathfrak{\Omega}_{0}$. Then $\tilde{\mathfrak{B}}^{2} \subseteq \boldsymbol{O}_{2}\left(\mathfrak{S}_{2} \mathfrak{O}_{0} \mathfrak{B}\right) \subseteq \mathfrak{I} \subseteq \mathfrak{S}$. Suppose $\mathfrak{B}^{2} \subseteq \mathfrak{S}$. Then

$$
\mathfrak{S} \mathfrak{Q}_{0} \mathfrak{B}=\left\langle\boldsymbol{O}_{2}\left(\mathfrak{C} \mathfrak{Q}_{0} \mathfrak{B}\right), \mathfrak{B}, \mathfrak{B}^{\mathfrak{Q}}\right\rangle \subseteq \mathfrak{S},
$$

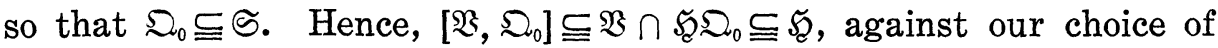
$\mathfrak{\Omega}_{0}$. So $\mathfrak{B}^{Q} \nsubseteq S$. By Lemma 13.41 , we get that $\mathfrak{B}$ is of type $\left(2,2^{b}\right)$ where $b \leqq 2$. Since $\left|\mathfrak{B}: \mathfrak{B}_{0}\right|=4$, and since $Z(\mathfrak{T}) \leqq O_{2}(\mathfrak{F})$, it follows that $\mathfrak{X}_{0} \neq 1$, so that $|\mathfrak{B}| \geqq 2^{3}$. Hence, $b=2$. By Lemma $13.42(\mathrm{~b})$, (c), $\tilde{\mathfrak{B}}$ is a four-group.

On the other hand, $\mathfrak{B} / \mathfrak{X}_{0}$ is faithfully represented on $\Omega$, so we can choose $\overline{\mathfrak{Q}}_{0}$ above, with additional property that $\Omega_{1}(\mathfrak{B})$ does not centralize $\overline{\mathfrak{Q}}_{0}$. In this case, $\tilde{\mathfrak{B}}$ is necessarily cyclic, against Lemma $13.42(\mathrm{c})$. We conclude that $\left|\mathfrak{B}: \mathfrak{X}_{0}\right|=2$.

Recall that $V \in \mathfrak{B}-\mathfrak{X}_{0}$. Choose $Q \in \mathfrak{\Omega}^{*}$ such that $\mathfrak{S} V$ inverts $\mathfrak{S} Q$. Hence, $Q \notin \mathfrak{S}$, so that $\mathfrak{B}^{Q} \cap \mathfrak{S}=\mathfrak{X}_{0}^{Q}$ is of index 2 in $\mathfrak{B}^{Q}$. By Lemma 13.42 , we get that $\mathfrak{B}$ is of type $\left(2,2^{b}\right)$ with $b \leqq 2$.

Case 1. $b=1$.

In this case, $[\mathfrak{V}, \mathfrak{I}] \leqq \mathfrak{X}_{0}$, a group of order 2 . Since $\mathfrak{B}$ does not 
centralize $\mathfrak{F}$, we get $[\mathfrak{B}, \mathfrak{S}]=\mathfrak{X}_{0}$, so that $\mathfrak{X}_{0}=Z(\mathfrak{I})$, the equality holding since by construction, $\boldsymbol{Z}(\mathfrak{I}) \subseteq \mathfrak{B}$. If $\mathfrak{X}_{0} \subseteq D(\mathfrak{S})$, then $V$ centralizes $\overline{\mathfrak{S}}=\mathfrak{S} / \boldsymbol{D}(\mathfrak{S})$, against $\mathfrak{B} \nsubseteq \mathfrak{S}$. Hence, $\mathfrak{X}_{0} \nsubseteq \boldsymbol{D}(\mathfrak{S})$. Since $\mathfrak{X}_{0}$ is the only minimal normal subgroup of $\mathfrak{I}$, we get $\boldsymbol{D}(\mathfrak{S})=1$.

Since $V$ centralizes, a hyperplane of $\mathfrak{K}$, and since $V$ also inverts $\mathfrak{S} \mathfrak{S} / \mathfrak{S} D(\Omega)$, it follows that $|\mathfrak{\Omega}|=3,|\mathfrak{T}: \mathfrak{F}|=2, \mathfrak{I}=\mathfrak{S}\langle V\rangle$. Since $\mathfrak{X}_{0}=Z(\mathfrak{I})$, it follows that $\mathfrak{I}$ is dihedral of order $2^{3}$, against $2 \in \pi_{4}$.

Case 2. $b=2$, and $\mathfrak{S}$ is not elementary.

Let $\mathfrak{S}_{0}=\boldsymbol{D}(\mathfrak{S}) \neq 1$, and let $\mathfrak{X}_{1}=\mathfrak{S}_{0} \cap \mathfrak{B}=\mathfrak{S}_{0} \cap \mathfrak{X}_{0}$. Since $\mathfrak{S}_{0} \cap$ $Z(\mathfrak{I}) \subseteq \mathfrak{B}$, we get $\mathfrak{X}_{1} \neq 1$. If $\mathfrak{X}_{1}=\mathfrak{X}_{0}$, then we get that $[\mathfrak{S}, \mathfrak{B}] \subseteq \mathfrak{S} \cap$ $\mathfrak{B}=\mathfrak{X}_{0}=\mathfrak{X}_{1} \subseteq \boldsymbol{D}(\mathfrak{S})$, against $V \notin \mathfrak{S}_{\text {。 }}$ Hence, $\left|\mathfrak{X}_{1}\right|=2$, since $\mathfrak{X}_{0}$ is a fourgroup and $1 \subset \mathfrak{X}_{1} \subset \mathfrak{X}_{0}$.

Let $\overline{\mathfrak{S}}_{0}=[\mathfrak{F}, V] \boldsymbol{D}(\mathfrak{S}) / \boldsymbol{D}(\mathfrak{S}) \leqq \mathfrak{X}_{0} \boldsymbol{D}(\mathfrak{S}) / \boldsymbol{D}(\mathfrak{S})$, so that $\overline{\mathfrak{S}}_{0}$ is of order 2 . Since $V$ centralizes a hyperplane of $\overline{\mathscr{S}}$ and $V$ inverts $\mathscr{S} \cong / \mathscr{S}_{\mathfrak{D}} D(\Omega)$, we again get $|\mathfrak{S}|=3, \mathfrak{I}=\mathfrak{S}\langle V\rangle$.

Let $\mathfrak{S}_{1}=[\mathfrak{S}, \mathfrak{\Omega}]$. Since $\mathfrak{N}=\mathfrak{S C}_{\mathfrak{R}}(\mathfrak{\Omega})$, we get $\mathfrak{S}_{1} \triangleleft \mathfrak{N}$. Let $\mathfrak{S}_{2}=$

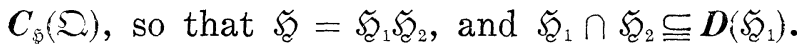

First, suppose $\mathfrak{S}_{1}$ is not elementary. In this case, we get $\boldsymbol{D}\left(\mathfrak{S}_{1}\right) \cap$ $\mathfrak{B}=\mathfrak{X}_{1}$ of order 2 , and $V$ centralizes a hyperplane of $\mathfrak{S}_{1} / \boldsymbol{D}\left(\mathfrak{S}_{1}\right)$. The only possibility is that $\mathfrak{F}_{1}$ is a quaternion group. Hence, $\mathfrak{X}_{1}=\boldsymbol{D}\left(\mathfrak{F}_{1}\right) \triangleleft \mathfrak{N}$, against $C\left(\mathfrak{x}_{1}\right) \leqq \subseteq$.

We may therefore assume that $\mathscr{S}_{1}$ is elementary. Let $\mathscr{S}_{3}=\mathscr{S}_{2} \cap$

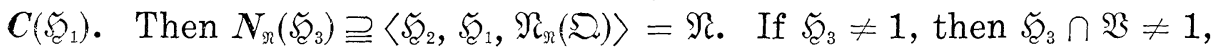
so that $\mathfrak{C} \subseteq\left(V_{0}\right) \subseteq \subseteq$ for some $V_{0}$ in $\mathfrak{B}^{\sharp}$. This is not the case, so $\mathfrak{F}_{3}=1$. If $\mathfrak{F}_{1}$ is a minimal normal subgroup of $\mathfrak{R}$, then $\mathscr{S}_{2}$ centralizes $\mathfrak{F}_{1}$, so $\mathfrak{S}_{2}=\mathscr{F}_{3}=1$ and $\mathfrak{I}$ is dihedral of order $2^{3}$. This is not the case since $\mathfrak{S C}_{\mathfrak{S}}$ is not elementary. Hence, $\mathfrak{F}_{1}$ is not a minimal normal subgroup of $\mathfrak{R}$.

Since $\left[\mathfrak{S}_{1}, V\right] \subseteq \mathfrak{X}_{0}$, it follows that $\left|\mathfrak{S}_{1}\right|=2^{4}, \mathfrak{S}_{1}=\mathfrak{X}_{0} \times \mathfrak{X}_{0}^{Q}$, where $Q$ is a generator for $\Omega$. Since $\mathfrak{S}_{3}=1$, it follows that $\left|\mathfrak{K}_{2}\right| \leqq 2$. Since, $\mathfrak{\mathfrak { S }}$ is not elementary, we get $\left|\mathfrak{F}_{2}\right|=2$. Hence, $|\mathfrak{T}|=2^{6}$. Let $\mathfrak{K}_{1} \supset \mathfrak{S}_{11} \supset 1$ be a composition series for $\mathscr{S}_{1}$ as $\mathfrak{N}$-module. Thus, $\left[\mathfrak{F}_{2}, \mathfrak{S}_{1}\right]=\mathfrak{F}_{11}$. By Hypothesis 13.3 (c), we have $\mathfrak{I} \subset \mathfrak{S}$. Let $\mathfrak{S}=\mathfrak{I} \Re$, where $\Re$ is a $S_{2^{\prime}}$ subgroup of $\subseteq$. Suppose $\mathfrak{I} \triangleleft \subseteq$. Since $\mathfrak{B}$ is of type $(2,4), \Re$ centralizes $\mathfrak{B}$. Since $\Re$ is faithfully represented on $\mathfrak{I} / \mathfrak{T}^{\prime}, \mathfrak{R}$ is also faithfully represented on $\mathfrak{I} / \mathfrak{T}^{\prime} \mathfrak{B}$. By the preceding paragraph, $\mathfrak{T} \mathfrak{i} \supseteqq\left\langle\mathfrak{B}, \mathfrak{F}_{11}\right\rangle$, a group of order $2^{4}$. Hence, $\left|\mathfrak{I}^{\prime} \mathfrak{B}\right|=2^{4}$, and $\mathfrak{I} / \mathfrak{T}^{\prime} \mathfrak{B}$ is a four-group. Hence, $|\mathfrak{R}|=3$. Since $\Re$ centralizes $\mathfrak{B}$ and normalizes $\mathfrak{I}^{\prime} \mathfrak{B}, R$ centralizes $\mathfrak{I}^{\prime} \mathfrak{B}$. Thus, $\mathfrak{I}^{\prime} \mathfrak{B}=\boldsymbol{C}_{\mathfrak{x}}(\mathfrak{R})$. Let $\mathfrak{I}_{0}=[\mathfrak{I}, \mathfrak{R}] \triangleleft \mathfrak{I}$. Thus, $\mathfrak{I}_{0}$ is a quaternion group, and $\mathfrak{I}=\mathfrak{I}_{0} \mathfrak{I}_{1}$, where $\mathfrak{I}_{1}=\boldsymbol{C}_{\mathfrak{S}}(\mathfrak{R})=\mathfrak{I}^{\prime} \mathfrak{B}$. Hence, $\mathfrak{I} \cap \boldsymbol{C}(\mathfrak{B}) \supseteq\left\langle\mathfrak{B}, \mathfrak{I}_{0}\right\rangle$, since $\mathfrak{I}_{0} \cap \mathfrak{I}_{1}=\mathfrak{I}_{0}^{\prime}$ is of order 2. Hence, $\left|\boldsymbol{A}_{\Theta}(\mathfrak{B})\right|=2$. This is not the case, since $C_{\mathfrak{F}_{1}}(\mathfrak{B})=\mathfrak{X}_{0}$ and $\left|\mathfrak{S}_{1}\right|=2^{4}$, Hence, $\mathfrak{I} \sharp \subseteq$. 
Let $\Re=\boldsymbol{O}_{2}(\mathfrak{S})$, so that $\mathfrak{B} \cong \Re$. Since $\Re$ centralizes $\mathfrak{B}$ and $\mathfrak{R}$ is faithfully represented on $\Re$, we get $|\Re|=2^{5},|\Re|=3$. Let $\Re_{0}=[\Re, \Re]$. Since every normal abelian subgroup of $\subseteq$ is generated by 2 elements, it follows that $\Re_{0}$ is a quaternion group. Let $\Re_{1}=C_{\Re}(\Re)$, so that $\Re=\Re_{0} \Re_{1}, \Re_{0} \cap \Re_{1}=\Re_{0}^{\prime}$. Hence, $\Re_{1}=\mathfrak{B}$, since $\mathfrak{B} \subseteq C_{\mathfrak{R}}(\Re)=\Re_{1}$, and since $|\mathfrak{B}|=\left|\Re_{1}\right|=2^{3}$. Since $\Re_{1}=\mathfrak{B}$, and $\Re_{0}$ centralizes $\Re_{1}$, we get $\left|A_{\mathscr{G}}(\mathfrak{B})\right|=2$. This is not the case, since $C_{\mathfrak{y}_{1}}(\mathfrak{B})=\mathfrak{X}_{0}$, and $\left|\mathfrak{S}_{1}\right|=2^{4}$.

Case 3. $b=2$ and $\mathfrak{S}$ is elementary.

We will show that $|\mathfrak{Q}|=3$ or 5 . Namely, $[\mathfrak{S}, \mathfrak{B}] \subseteq \mathfrak{X}_{0}$, so $q=3$ or 5. Hence, it suffices to show that $|\mathfrak{Q}|=q$. Suppose $\mathfrak{Q}$ is elementary. Then $V$ inverts $\mathfrak{S} \Omega / \mathscr{E}$, so if $Q \in \mathfrak{Q}^{\sharp}, C_{\mathfrak{F}}(\mathfrak{\Omega})$ admits $V$. Since $\mathfrak{\Omega} \cap \mathfrak{S}=1$, it follows that $\left[C_{\mathfrak{p}}(\mathfrak{\Omega}), V\right] \subseteq C_{\mathfrak{p}}(Q) \cap \mathfrak{B}=1$. If $|\mathfrak{Q}|=q^{2}$, we get that $\mathfrak{S}=\left\langle C_{\mathfrak{p}}(Q) \mid Q \in \mathfrak{Q}^{\sharp}\right\rangle \leqq C(V)$, against $V \notin \mathscr{S}$. Hence, if $\mathfrak{Q}$ is elementary, then $|\mathfrak{Q}|=q$. Suppose $\mathfrak{Q}$ is not elementary, so that $\mathfrak{Q}$ is extra special of order $q^{3}$. Since $\mathfrak{B}$ centralizes $\mathfrak{S} \mathfrak{Q}^{\prime} / \mathfrak{S}$, it follows that $\mathfrak{Q}^{\prime}$ normalizes $[\mathfrak{S}, \mathfrak{B}]=\mathfrak{X}_{0}$. Hence, $\mathfrak{Q}^{\prime} \subseteq \mathfrak{S}$, since $\mathfrak{B}$ is a T. I. set in $\mathfrak{B}$. Since $\mathfrak{B}$ is of type $(2,4), \mathfrak{Q}^{\prime}$ centralizes $\mathfrak{B}$, in particular centralizes $\mathfrak{X}_{0}$.

Let $\mathfrak{S}_{0}=\left[\mathfrak{S}_{2}, \mathfrak{\Omega}^{\prime}\right]$. Since $\mathfrak{\Omega}^{\prime} \neq 1, \mathfrak{S}_{0} \neq 1$. Since $\mathfrak{X}_{0} \subseteq C\left(\mathfrak{\Omega}^{\prime}\right)$, we get $\mathfrak{S}_{0} \cap \mathfrak{X}_{0}=1$. Hence, $\mathfrak{S}_{0} \cap \boldsymbol{Z}(\mathfrak{T})=1$, since $\boldsymbol{Z}(\mathfrak{T}) \subseteq \mathfrak{B}$. This is impossible since $\mathfrak{S}_{0} \triangleleft \mathfrak{T}$. We conclude that $|\mathfrak{Q}|=3$ or 5 .

Since $C_{\mathfrak{F}}(\mathfrak{Q}) \triangleleft \mathfrak{N}$, and since $\boldsymbol{C}_{\mathfrak{F}}(\mathfrak{Q}) \cap \mathfrak{B}=1$, it follows that $\boldsymbol{C}_{\mathfrak{F}}(\mathfrak{Q})=1$. Since $\left[\mathfrak{B}, \mathfrak{S}_{\mathfrak{C}}\right] \subseteq \mathfrak{B}_{0}$ and $\mathfrak{B}_{0}$ is a four-group, it follows that $\left|\mathfrak{S}_{\mathfrak{C}}\right|=2^{4}$. Hence, $|\mathfrak{I}|=2^{a}$, with $5 \leqq a \leqq 6$. If $a=5$, we get $\mathfrak{I} \triangleleft \mathfrak{S}$, since $|\mathfrak{T}: \mathfrak{B}|=4$ and a $S_{2}$-subgroup $\mathfrak{S}_{2^{\prime}}$ of $\mathfrak{S}$ is represented faithfully on $\boldsymbol{O}_{2}(\mathfrak{S})$. But $\mathfrak{F}$ is the only subgroup of $\mathfrak{I}$ of its isomorphism type, so $\mathfrak{K} \triangleleft \mathfrak{S}$. This is impossible, by Hypothesis $13.3(\mathrm{c})$, so we get $a=6$. Thus, $|\mathfrak{Q}|=5$, and $\mathfrak{I} / \mathfrak{F}$ is cyclic of order 4 .

Suppose $\mathfrak{S} \cong \boldsymbol{O}_{2}(\mathfrak{S})$. Since $\mathfrak{S}$ is the only subgroup of $\mathfrak{I}$ of its isomorphism class, we get $\mathfrak{S} \triangleleft \mathfrak{S}$, against Hypothesis 13.3 (c). Thus, $\mathfrak{S} \nsubseteq \boldsymbol{O}_{2}(\mathfrak{S})$. This implies that $\left|\boldsymbol{O}_{2}(\mathfrak{S})\right|=2^{5}$. Let $\mathfrak{S}_{2}$, be a $S_{2}$-subgroup of $\mathfrak{S}$, so that $\left|\mathfrak{S}_{2^{\prime}}\right|=3$. Let $J$ be an element of $\mathfrak{S}-\boldsymbol{O}_{2}(\mathfrak{S})$. We may assume that $J$ inverts a generator $S$ of $\mathfrak{S}_{2^{\prime}}$. Thus, $\mathfrak{I}_{1}=\left[\boldsymbol{O}_{2}(\mathfrak{S}), \mathfrak{S}_{2^{\prime}}\right]$ admits $J$. Since $\left[\mathfrak{I}_{1}, J\right] \subseteq \mathfrak{I}_{1} \cap \mathfrak{F}_{\mathcal{E}}$, it follows that $\mathfrak{I}_{1}$ is not a quaternion group. Since $\left|\mathfrak{I}_{1}: \mathfrak{I}_{1} \cap \boldsymbol{C}\left(\mathfrak{S}_{2^{\prime}}\right)\right| \leqq 4$, it follows that $\mathfrak{I}_{1}$ is a four-group. This is impossible, since $\mathfrak{I}_{1} \triangleleft \mathfrak{S}$, and since $\mathfrak{S}_{2^{\prime}}$ centralizes $\mathfrak{B} \supseteqq \boldsymbol{Z}(\mathfrak{T})$. The proof is complete.

Let $\mathscr{R}=\boldsymbol{O}_{2}(\mathfrak{S})$, and let $\mathscr{S}=\mathscr{R} \cap \boldsymbol{C}(\mathfrak{B})$. Since $\mathfrak{B}$ is a normal abelian subgroup of $\mathfrak{S}$ of maximal order, it follows that $\mathfrak{B}=Z(\mathfrak{S})$. Let $c$ be the class of nilpotency of $\mathfrak{S}$. If $c>2$, then $C_{c-1}(\mathfrak{S})$ is abelian, so that $\mathfrak{B} C_{c-1}(\mathfrak{S})$ is a normal abelian subgroup of $\mathfrak{S}$ which contains $\mathfrak{B}$ properly. 
This is impossible by maximality of $\mathfrak{B}$, so $c \leqq 2$. Let $\mathfrak{S}_{2^{\prime}}$ be a $S_{2^{\prime}}$ subgroup of $\mathfrak{S}$.

LEMMA 13.45.

(a) $\mathfrak{S} / \mathfrak{B}$ is elementary.

(b) $\mathfrak{S}_{2}$, is faithfully represented on $\mathfrak{S}$.

Proof. Since $c \leqq 2, \mathfrak{S} / \mathfrak{B}$ is abelian of type $\left(2^{a_{1}}, \cdots, 2^{a_{2}}\right), a_{1} \leqq a_{2} \leqq$ $\cdots \leqq a_{s}$. Suppose $a_{s}=a \geqq 2$. Let $\mathscr{S}_{0}=\mho^{a-1}(\mathfrak{C} \bmod \mathfrak{B}) \supset \mathfrak{B}$. If $H_{1}, H_{2} \in$ $\mathfrak{S E}$, then

$$
\left[H_{1}^{2^{a-1}}, H_{2}^{2^{a-1}}\right]=\left[H_{1}^{2^{a}}, H_{2}^{2^{a-2}}\right]=1,
$$

since $H_{1}^{2^{a}} \in \mathfrak{B}$. Thus, $\mathfrak{F}_{0}$ is abelian, against the maximality of $\mathfrak{B}$. This establishes (a).

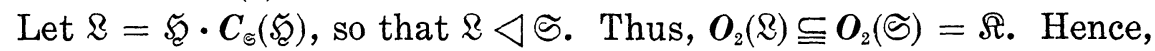
$\mathfrak{F}$ is the $S_{2}$-subgroup of $\mathbb{R}$. Thus, $\mathbb{R}=\mathfrak{F} \times \boldsymbol{O}_{2^{\prime}}(\mathbb{R})$, since $\boldsymbol{A}_{\mathfrak{\&}}(\mathfrak{E})=\boldsymbol{I}(\mathfrak{E})$. Since $2 \in \pi_{4}, O_{2^{\prime}}(\mathfrak{S})=1$, so $O_{2^{\prime}}(\mathbb{R})=1$, establishing (b).

Lemma 13.46. Suppose $\mathfrak{B} \cong \mathfrak{S}_{1} \subset \mathfrak{S}$ and $\left|\mathfrak{S}_{2}: \mathfrak{S}_{1}\right|=2$. Then every involution of $\boldsymbol{C}_{\mathfrak{\Im}}\left(\mathfrak{S}_{1}\right)$ is contained in $\Re$.

Proof. Suppose $X$ is an involution of $C_{\mathfrak{F}}\left(\mathscr{S}_{1}\right)-\Re$. Then $\subseteq$ contains an element $Q$ of odd prime order which is inverted by $X$, by Lemma 5.36. Let $\mathfrak{D}=\langle Q, X\rangle, \mathfrak{R}=\mathfrak{S C D}_{\mathfrak{D}}$. Let $\mathfrak{S}^{*}=\left[\mathfrak{S}_{2},\langle Q\rangle\right], \mathfrak{S}_{1}^{*}=$ $\boldsymbol{C}_{\mathfrak{F}}(Q)$. Thus, $\mathfrak{S}^{*}$ and $\mathfrak{S}_{1}^{*}$ admit $\mathfrak{D}$, and $\mathfrak{B} \cong \mathfrak{F}_{1}^{*}$. Since $\langle Q\rangle$ is represented faithfully on $\mathfrak{H C}_{2}$, we get $\mathfrak{S C}^{*} \neq 1$. Thus, $\mathfrak{S}^{*} \cap \mathfrak{S}_{1}$ is of index 2 in $\mathfrak{S}^{*}$, as is $\mathfrak{S}^{*} \cap \mathfrak{S E}_{1}^{Q}$. Since $\left\langle X, X^{Q}\right\rangle=\mathfrak{D}$ centralizes $\mathfrak{S E}^{*} \cap \mathfrak{S}_{1} \cap \mathfrak{S}_{1}^{Q}$, it follows that $Q$ centralizes a subgroup of $\mathfrak{K}^{*}$ of index 4 . Since $Q$ has no non identity fixed points on $\mathfrak{S}^{*} / \boldsymbol{D}\left(\mathfrak{S C}^{*}\right)$, it follows that $\mathfrak{S}_{\mathfrak{C}}^{*}$ is either a four-group or a quaternion group. If $\mathfrak{S}^{*}$ is quaternion, then $C_{\mathfrak{F}^{*}}(X)$ is of order 2, as is well known. Hence, $\mathfrak{S}^{*}$ is a four-group. On the other hand, $\mathfrak{S}^{*} \triangleleft \mathfrak{S}$, so $\mathfrak{S}=\mathfrak{S E}^{*} \times \mathfrak{S}_{1}^{*}$, which gives $\mathfrak{S}^{*} \subseteq Z(\mathfrak{S})=\mathfrak{B}$, against $\mathfrak{B} \subseteq \mathfrak{S}_{1}^{*}$. The proof is complete.

The next easy lemma is important, and perhaps will admit of interesting generalization in later work, since it involves the explicit construction of non solvable local subgroups.

LEMma 13.47. If $G \in \mathbb{S}-\mathfrak{S}$, then one of the following holds:

(a) $\mathfrak{B}_{0} \nsubseteq \mathfrak{S}^{G}$,

( b ) $\mathfrak{B}_{0}^{G} \nsubseteq \mathfrak{S}_{\mathfrak{c}}$.

Proof. Suppose false. Let $\mathfrak{Y}=\left\langle\mathfrak{B}_{0}, \mathfrak{B}_{0}^{G}\right\rangle$. Thus, $\mathfrak{B}_{0} \triangleleft \mathfrak{Y}, \mathfrak{B}_{0}^{G} \triangleleft \mathfrak{Y}$, since $\mathfrak{Y} \subseteq \mathfrak{S C}_{\mathfrak{C}} \cap \mathfrak{S}^{G}$. Since $\mathfrak{B}$ is a T.I. set in $\mathfrak{B}$, we get $\mathfrak{Y}=\mathfrak{B}_{0} \times \mathfrak{B}_{0}^{G}$, 
an elementary group of order $2^{4}$. Since the normal closure of $\mathfrak{Y}$ in $\subseteq$ is a subgroup of $\mathfrak{K}$, Hypothesis 13.3 (c) implies that $\mathfrak{Y}^{S}$ is non abelian. Choose $S$ in $\mathcal{S}$ such that $\left\langle\mathfrak{Y}, \mathfrak{Y}^{S}\right\rangle$ is non abelian.

Since $\boldsymbol{D}(\mathfrak{K}) \subseteq \mathfrak{B}=\boldsymbol{Z}(\mathfrak{E})$, it follows that $\mathfrak{S E}^{\prime} \subseteq \mathfrak{B}_{0}=\Omega_{1}(\mathfrak{B})$. Thus, $\mathfrak{Y}\left\langle\left\langle\mathfrak{Y}, \mathfrak{Y}^{S}\right\rangle\right.$ and $\mathfrak{Y}^{S}$ stabilizes the chain $\mathscr{C}: \mathfrak{Y} \supset \mathfrak{B}_{0} \supset 1$. Choose $A \in$ $\mathfrak{B}_{0}^{G S^{\sharp}} \subseteq \mathfrak{Y}^{S}$. Suppose $C(A) \cap \mathfrak{B}_{0}^{G} \neq 1$. Then $A \in \mathfrak{S}^{G}$, by Hypothesis $13.1(\mathrm{~d})$. Hence, $\left[\mathfrak{B}_{0}^{G}, A\right] \subseteq \mathfrak{B}_{0}^{G} \cap \mathfrak{B}_{0}=1$, so that $A$ centralizes $\mathfrak{Y}$. If $\mathfrak{B}_{0}^{G S}=\mathfrak{B}_{0}$, then $G S \in N\left(\mathfrak{B}_{0}\right)=\mathfrak{S}$, so that $G \in \mathfrak{S}$. This is not the case, so $\mathfrak{B}_{0}^{G S} \cap$ $\mathfrak{B}_{0}=1$. This implies that $\mathfrak{B}_{0}^{G S}$ centralizes $\mathfrak{Y}$. Since $\mathfrak{Y}^{S}=\mathfrak{B}_{0}^{G S} \times \mathfrak{B}_{0}$, we get that $\left\langle\mathfrak{Y}, \mathfrak{Y}^{S}\right\rangle$ is abelian, against our construction. Hence, $C(A) \cap \mathfrak{B}_{0}^{G}=1$ for all $A \in \mathfrak{B}_{0}^{G S \#}$. Let $\mathfrak{B}=A_{\circledast}(\mathscr{C}) \supseteqq A \mathfrak{B}_{0}^{G S}(\mathscr{C})$.

Similarly, let $\mathscr{C}^{*}$ be the chain $\bigvee \supset \mathfrak{B}_{0}^{G} \supset 1$. We can choose $S^{*}$ in $\mathfrak{S}^{a}$ such that $\left\langle\mathfrak{Y}, \mathfrak{Y}^{S^{*}}\right\rangle$ is not abelian. Hence, $\mathfrak{Y}_{0}^{s^{*}}$ stabilizes $\mathscr{C}^{*}$ and does not centralize $\mathfrak{Y}$. It follows that $\boldsymbol{A}_{\mathbb{\Theta}}(\mathfrak{Y})$ is non solvable. This contradiction completes the proof.

Lemma 13.48. Suppose $G \in \mathbb{B}$ and the following hold:

(a) $\mathfrak{B}^{G} \cap \mathfrak{S} \neq 1$.

( b ) $\mathfrak{B}_{0}^{G} \nsubseteq \mathfrak{S}$.

Then $\mathfrak{B}=\mathfrak{B}_{0}$.

Proof. Let $\mathfrak{X}=\mathfrak{B}^{G} \cap \mathfrak{S}$, so that $\mathfrak{X} \neq 1$. Suppose $X \in \mathfrak{X}^{\#}$ and $|\mathfrak{B} \cap \boldsymbol{C}(X)| \geqq 4$. Since $\boldsymbol{C}(X) \subseteq \mathfrak{S}^{G}$, we get $\mathfrak{B} \cap \boldsymbol{C}(X) \leqq \mathfrak{\Im}^{G}$. Since $|\mathfrak{B} \cap \boldsymbol{C}(X)| \geqq 4$, we can choose $\boldsymbol{V}$ in $\mathfrak{B} \cap \boldsymbol{C}(X) \cap \boldsymbol{C}\left(\mathfrak{B}_{0}^{G}\right)^{\sharp}$. Hence, $\mathfrak{B}_{0}^{G} \subseteq$ $C(V) \subseteq \subseteq$, against (b). Hence, $|\mathfrak{X}|=2$ and $\left|\boldsymbol{C}_{\mathfrak{B}}(X)\right|=2$. This implies that $\mathfrak{B X}$ is of maximal class, forcing $\mathfrak{B}=\mathfrak{B}_{0}$.

Lemma 13.49. Assume that $G \in \mathbb{B}-\mathcal{S}$ and the following hold:

(a) $\mathfrak{B}_{0}^{G} \subseteq \mathfrak{T}$.

(b) $\mathfrak{B}_{0}^{G} \cap \mathfrak{S}_{\mathfrak{E}}=\mathfrak{X}_{0}$ is of order 2 .

(c) $\mathfrak{S}_{1}=C_{\mathfrak{E}}\left(\mathfrak{X}_{0}\right)$ is of index 2 in $\mathfrak{S}_{\text {. }}$.

(d) The normal closure of $\mathfrak{B}_{0}^{G}$ in $\mathfrak{T}$ is abelian.

Set $\mathbb{R}=\boldsymbol{O}_{2,2^{\prime}}(\mathfrak{S}) / \boldsymbol{O}_{2}(\mathfrak{S}), \mathfrak{B}_{0}^{G}=\mathfrak{X}_{0} \times \mathfrak{X}_{1}$, where $\mathfrak{X}_{i}=\left\langle X_{i}\right\rangle$ is of order 2. Then the following hold:

(i) If $\mathfrak{R}_{1}$ is any subgroup of \& inverted by $X_{1}$, then $\left|\mathfrak{R}_{1}\right|=1$ or 3.

(ii) If $Q$ is any non identity element of $\subseteq$ of odd order which is inverted by $X_{1}$, then $[\mathfrak{S},\langle Q\rangle]=\mathfrak{S}^{*}$ contains $X_{0}$ and is the central product of 2 quaternion groups. Furthermore, $\mathfrak{S}_{\mathfrak{C}}=\mathfrak{S}^{*} \boldsymbol{C}_{\mathfrak{F}}\left(\mathfrak{S}_{\mathcal{E}}^{*}\right)$, and $C_{\mathfrak{F}}\left(\mathfrak{S C}^{*}\right) \subseteq C\left(\mathfrak{B}_{0}^{G}\right)$.

(iii) $\mathfrak{B}=\mathfrak{B}_{0}$.

Proof. Let $Q$ and $\mathfrak{S}^{*}$ be as above. Let $\mathfrak{S}_{1}^{*}=\mathfrak{S}^{*} \cap \mathfrak{S}_{1}$. Since $\boldsymbol{C}\left(X_{0}\right) \subseteq \widetilde{S}^{G}$, we get $\left[\mathfrak{F}_{1}^{*}, X_{1}\right] \subseteq \mathfrak{F}_{\mathcal{E}} \cap \mathfrak{B}_{0}^{G}=\mathfrak{X}_{0}$. Since $\langle Q\rangle=\mathfrak{Q}$ has no 
non identity fixed points on $\mathfrak{S C}^{*} / \boldsymbol{D}\left(\mathfrak{S}^{*}\right)$, it follows that $\mathfrak{S}^{*} / \boldsymbol{D}\left(\mathfrak{S}^{*}\right)$ is a free $F_{2}\left\langle X_{1}\right\rangle$-module. Let $\mathfrak{F}=\left[\mathfrak{S}^{*}, X_{1}\right]$. By $(\mathrm{d})$, $\mathfrak{F}$ is elementary, and by the preceding remark, we get $\mathfrak{F}^{*}=\left\langle\mathfrak{F}, \mathfrak{F}^{Q}\right\rangle$, since $\left\langle\mathfrak{F}^{2}, \mathfrak{F}^{Q}\right\rangle$ covers $\mathfrak{S}^{*} / \boldsymbol{D}\left(\mathfrak{S}^{*}\right)$.

First, suppose $\mathfrak{F}_{\mathfrak{F}}$ is of order 2. In this case, $\left|\mathfrak{S}^{*}: D\left(\mathfrak{S}^{*}\right)\right|=4$, and since $\mathfrak{S}_{2}^{*}$ is generated by involutions, we get that $\mathfrak{S}^{*}$ is a fourgroup. Since $\mathfrak{S C}^{*}=[\mathfrak{S}, \mathfrak{⿰}] \triangleleft \mathfrak{F}$, we get $\mathfrak{K}^{*} \subseteq \mathbb{Z}(\mathfrak{S})=\mathfrak{B}$. On the other hand, $\mathfrak{B} \subseteq C\left(X_{0}\right) \subseteq \subseteq^{G}$, so that $\left[\mathfrak{B}^{G}, \mathfrak{B}\right]=1$, by Lemma 13.41 applied to $\mathfrak{S}^{a}$. Thus, $X_{1}$ centralizes $\mathfrak{B}$, so $\mathfrak{Q}$ also centralizes $\mathfrak{B}$. This contradiction show that $|\mathfrak{F}| \geqq 4$. In particular, since $\left[\mathfrak{F}_{1}^{*}, X_{1}\right] \leqq \mathfrak{X}_{0}$, we get that $\left|\mathfrak{S}^{*}: \mathfrak{S}_{1}^{*}\right|=2$, and $\left[\mathfrak{S}_{1}^{*}, X_{1}\right]=\mathfrak{X}_{0}$. Hence, $\mathfrak{S}^{*}$ contains $\mathfrak{X}_{0}$.

Let $\mathfrak{F}_{1}=\mathfrak{F} \cap \mathfrak{S}^{* *}$. If $\left|\mathfrak{F}_{:}: \mathfrak{F}_{1}\right| \leqq 2$, then $\left|\mathfrak{S C}^{*}: \mathfrak{S C}^{* \prime}\right|=4$, against our previous argument. Hence, $\left|\mathfrak{s}_{:} \mathfrak{\xi}_{1}\right| \geqq 4$. Let $\mathfrak{F}=\mathfrak{F}_{1} \times \mathfrak{F}_{2}$ where $X_{0} \in$ $\mathfrak{F}_{2} ; \mathfrak{F}_{2}$ is available since $X_{0} \notin \mathfrak{S}^{* \prime}$ and $X_{0} \in \mathfrak{F}$, while $\mathfrak{F}$ is elementary.

Choose $H \in \mathfrak{S}^{*}-\mathfrak{S}_{1}^{*}$. Thus, $\left\langle\left[H, X_{1}\right], X_{0}\right\rangle$ covers $\mathfrak{F} / \mathfrak{S}_{1}$, and so $\left|\widetilde{\mathfrak{F}}_{2}\right|=4$. Hence, $\left|\mathfrak{S C}^{*}: \boldsymbol{D}\left(\mathfrak{S}_{2}^{*}\right)\right|=2^{4}$, since $\left\langle\mathfrak{F}_{2}, \mathfrak{F}_{2}^{Q}\right\rangle=\mathfrak{S C}^{*}$. Since $\mathfrak{S}_{1}^{*} \subset \mathfrak{S}_{2}^{*}$, it follows that $\mathfrak{S}^{*}$ is non abelian. Let $\mathfrak{F}$ be a subgroup of $\mathfrak{S}^{* \prime}$ of index 2. Then $\mathfrak{F} \mathfrak{F} / \mathfrak{F}$ is elementary of order 8 , and so $\mathfrak{S}^{*} / \mathfrak{F}$ is the central product of 2 quaternion groups. Hence, Aut $\left(\mathfrak{S}^{*} / \mathfrak{F}\right)$ is a 2,3group whose $S_{3}$-subgroups are elementary, and so $Q$ has order 3 .

Since $Q$ has no fixed points on $\mathfrak{S C}^{*}-\mathfrak{S C}^{* \prime}$, it follows that for each $H \in \mathfrak{S}^{*}, H \cdot H^{Q} \cdot H^{Q^{2}} \in \mathfrak{S}^{* \prime}$. This implies that for each involution $I$ of $\mathfrak{S}^{*},\left[I, I^{Q}\right]=1$. Let $\mathfrak{F}_{2}=\left\langle X_{0}\right\rangle \times\left\langle Y_{0}\right\rangle$. Then $\mathfrak{S}^{*}=\left\langle\mathfrak{F}_{2}, \mathfrak{F}_{2}{ }^{Q}\right\rangle$ and

$$
\left[X_{0}, X_{0}^{Q}\right]=\left[Y_{0}, Y_{0}^{Q}\right]=\left[X_{0} Y_{0},\left(X_{0} Y_{0}\right)^{Q}\right]=1 \text {, }
$$

so that $\mathfrak{S}^{* \prime}=\left\langle\left[X_{0}, Y_{0}^{Q}\right]\right\rangle$ is of order 2 , so that $\mathfrak{F}=1$.

Since $\mathfrak{B} \cap \mathfrak{S C}^{*}=\mathfrak{S}_{2}{ }^{* \prime}$, it follows that $\mathfrak{S}$ stabilizes $\mathfrak{S}^{*} \supset \mathfrak{S}_{\mathfrak{C}}{ }^{\prime \prime} \supset 1$. Hence, $C_{\mathfrak{5}}(Q)$ centralizes $\mathfrak{S E}^{*}$, and $C_{\mathfrak{5}}(Q)=C_{\mathfrak{F}}\left(\mathfrak{S C}^{*}\right), \mathfrak{S}=\mathfrak{S}^{*} \boldsymbol{C}_{\mathfrak{5}}\left(\mathfrak{S E}^{*}\right)$. Since $\boldsymbol{C}_{\mathfrak{1}}\left(\mathfrak{S E}^{*}\right) \subseteq \boldsymbol{C}\left(X_{0}\right) \subseteq \Im^{G}$, we get $\left[\boldsymbol{C}_{\mathfrak{\wp}}\left(\mathfrak{S E}^{*}\right), \mathfrak{B}^{G}\right] \subseteq \mathfrak{X}_{0} \cap \boldsymbol{C}\left(\mathfrak{S}^{*}\right)=1$. This establishes (ii).

Set $X_{v}^{*}=X_{0}^{Q}$. Thus, $Q$ normalizes $\left\langle X_{0}, X_{0}^{Q}, \mathfrak{S}^{* \prime}\right\rangle$, an elementary group of order 8. Since $Q$ does not centralize $\left\langle X_{0}, X_{0}^{*}, \mathfrak{S C}^{* \prime}\right\rangle$, it follows that $\left[X_{1}, X_{0}^{*}\right] \neq 1$. Thus, $X_{0}^{*} \in C\left(X_{0}\right) \subseteq \subseteq^{G}$, but $\mathfrak{B}_{0}^{G} \nsubseteq \Im^{G Q}$, this last relation holding since $\left[\mathfrak{S}_{0}^{G}, X_{0}^{*}\right] \subseteq \mathfrak{B}_{0}^{G}$, while $\mathfrak{B}_{0}^{G} \neq \mathfrak{B}_{0}^{G Q}$, this final inequality holding since $X_{1}$ inverts $Q$. By Lemma 13.48 with $\varsigma^{G Q}$ in the role of $\subseteq$ and our present $G$ in the role of $G$, we get $\mathfrak{B}=\mathfrak{B}_{0}$; so (iii) holds.

It remains to prove (i). Since $Q$ has order 3 , it follows that $\mathbb{Z}_{1}$ is an elementary 3 -group. Since $X_{1}$ inverts $\mathfrak{L}_{1}$, it follows that $\mathfrak{\Omega}_{1}=$ $\mathfrak{2} \boldsymbol{O}_{2}(\mathfrak{S}) / \boldsymbol{O}_{2}(\mathfrak{S})$, where $\mathfrak{A}$ is a 3-group inverted by $X_{1}$. We assume without loss of generality that $Q \in \mathfrak{A}$. Thus, $\mathfrak{S}^{*}$ admits $\mathfrak{A}$. Since $C_{\tilde{F}^{*}}\left(X_{1}\right)$ is elementary of order $2^{3}, X_{1}$ interchanges the 2 quaternion subgroups of $\mathfrak{S}^{*}$. If $|\mathfrak{A}| \geqq 3^{2}$, we can choose $A$ in $\mathfrak{A}$ such that $\boldsymbol{C}(A) \cap \mathfrak{S}_{\mathcal{C}}^{*}$ is a quaternion group. Since $\boldsymbol{C}(A) \cap \mathfrak{S}^{*}$ admits $X_{1}$, we have a contradiction. Thus, $|\mathfrak{A}|=3$, and (i) holds. 
Lemma 13.50. Assume that $G \in \mathbb{B S}-\mathfrak{S}$ and the following hold:

(a) $\mathfrak{S}^{G} \cap \mathfrak{T}$ is of index 2 in $\mathfrak{S}^{G}$.

(b) $\mathfrak{B}^{G} \subseteq \mathfrak{T}$.

(c) $\mathfrak{B}_{0}^{G} \cap \mathfrak{S}_{\mathfrak{Z}}=\mathfrak{X}_{0}$ is of order 2.

(d) $\mathfrak{S}_{1}=\boldsymbol{C}_{\mathfrak{F}}\left(\mathfrak{X}_{0}\right)$ is of index 2 in $\mathfrak{S}_{\text {. }}$.

(e) The normal closure of $\mathfrak{B}_{0}^{G}$ in $\mathfrak{I}$ is abelian. Set $\mathfrak{R}=\mathfrak{S}^{G} \cap \mathfrak{T}, \Re_{0}=\Re \cap \Re$. Then

(i) $\Re_{0} \triangleleft \Re$, and $\Re / \Re_{0}$ contains no abelian subgroup of order $2^{3}$.

(ii) $\mathfrak{L}=\boldsymbol{O}_{2,2^{\prime}}$ (S) contains no elementary subgroup of order $3^{3}$.

Proof. Since $\Re \triangleleft \subseteq$, so also $\Re_{0} \triangleleft \Re$. Suppose (i) is false. For each subset $\mathfrak{S}_{0}$ of $\mathfrak{S}$, let $\overline{\mathfrak{S}}_{0}=\mathfrak{S}_{0} \Re / \Re$. Thus, $\bar{\Re}$ and $\Re / \Re_{0}$ are incident, so $\Re$ contains a subgroup $\Re_{1} \supset \Re_{0}$ such that $\bar{\Re}_{1}$ is abelian of order $2^{3}$.

Since the hypotheses of this lemma are stronger than those of Lemma 13.49 , we get $\mathfrak{B}=\mathfrak{B}_{0}$. Hence, $\mathfrak{F}$ is of exponent 4 , so $\mathfrak{R}$ is of exponent at most 4 . Hence, $\Re_{1}$ is either elementary or of type $(2,4)$. We assume without loss of generality that $\mathfrak{X}=\mathfrak{B}_{0}^{G} \subset \Re_{1}$ This assumption is permissible, since $X$ is a central subgroup of $\Re$ of order 4 . Let $\Re_{2}=\Omega_{1}\left(\Re_{1} \bmod \Re_{0}\right)$, so that $\Re_{2} \supseteqq \Omega_{1}\left(\Re_{1}\right) \supset \mathfrak{X}_{1}$. Here we have written $\mathfrak{X}=\mathfrak{X}_{0} \times \mathfrak{X}_{1}$, where $\mathfrak{X}_{i}=\left\langle X_{i}\right\rangle$ is of order 2 , and $\mathfrak{X}_{0}$ is given in (c).

Let $\mathfrak{R}_{0}=\boldsymbol{F}\left(\mathfrak{S} \bmod \boldsymbol{O}_{2}(\mathfrak{S})\right)$. By Lemma 13.49 , [ $\left.\overline{\mathfrak{Q}}_{0}, \overline{\mathfrak{X}}_{1}\right]$ is a 3-group. We first show that $\left[\overline{\mathfrak{R}}, \overline{\mathfrak{R}}_{2}\right]$ is a 3 -group. Suppose false. Let $\mathfrak{Q}$ be a

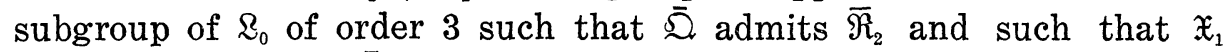
does not centralize $\overline{\mathfrak{D}}$. We assume without loss of generality that $X_{1}$ inverts a generator $Q$ of $\Omega$.

Let $\mathfrak{S}^{*}=[\mathfrak{K}, \mathfrak{O}]$, so that by Lemma 13.49 , $\mathfrak{S}^{*}$ is the central product of 2 quaternion groups. Since $\bar{\Omega}_{0}$ is nilpotent $\boldsymbol{C}_{\Omega_{0}}(Q)$ contains a $S_{\{2,3\}}$-subgroup $\mathfrak{D}$ of $\mathfrak{R}_{0}$. Hence, $\mathfrak{D}$ centralizes $\mathfrak{S}^{*}$, since Aut $\left(\mathfrak{S}^{*}\right)$ is a 2,3-group. Since $X_{0} \in \mathfrak{S}^{*}$, we ge $\mathfrak{D} \subseteq \mathfrak{S}^{G}$, so that $[\Re, \mathfrak{D}] \subseteq \mathfrak{S}^{G} \cap$ $\mathfrak{\Omega}_{0} \subseteq \Re$. Hence, $\left[\overline{\mathfrak{\Omega}}_{0}, \bar{\Re}_{2}\right]$ is a 3-group.

We next show that if $\Omega_{3}$ is a $S_{3}$-subgroup of $\mathfrak{\Omega}_{0}$, then

$$
\mathscr{S} \mathscr{C} \mathscr{N}_{3}\left(\mathbb{R}_{3}\right) \neq \varnothing \text {. }
$$

Suppose false. Then Aut $\left(\mathbb{2}_{3}\right)$ does not contain any non cyclic abelian subgroup of order 8 , against the fact that $\bar{\Re}_{1}$ acts faithfully on $\bar{\Omega}_{3}$. Hence, $\mathscr{S} \mathscr{C} \mathscr{N}_{3}\left(\mathfrak{R}_{3}\right) \neq \varnothing$. In particular, Hypothesis 13.1 is satisfied.

We next observe that since $\left\langle\bar{\Re}_{2}, \bar{\Omega}_{3}\right\rangle$ is supersolvable, $\mathfrak{\Omega}_{3}$ contains an elementary subgroup $\mathfrak{A}$ of order $3^{3}$ such that $\overline{\mathfrak{A}}$ admits $\bar{\Re}_{2}$ and such that $\bar{\Re}_{2}$ acts faithfully on $\overline{\mathfrak{A}}$. We may assume that $X_{1}$ inverts the element $Q$ of $\mathfrak{R}^{\#}$. Hence, $\mathfrak{S}^{*}=\left[\mathfrak{F}_{2},\langle Q\rangle\right]$ admits $\mathfrak{R}$, so $C_{\mathfrak{U}}\left(\mathfrak{S}^{*}\right) \neq 1$. Choose $A \in \mathfrak{I}^{*} \cap C\left(\mathfrak{S}^{*}\right)$. Since $X_{0} \in \mathfrak{S}^{*}$, we get $A \in \mathfrak{S}^{G}$. Since $C(A)=$ $C_{\mathfrak{\Phi}}(A) \in \mathscr{M}^{*}(\mathbb{S})$, it follows that $\mathfrak{S}_{3}$-subgroups of $\mathfrak{S} \cap \mathfrak{S}^{G}$ are non cyclic. This violates Theorem 13.1 (b)(ii) with $\subseteq$ in the role of $\mathfrak{M}$. The proof of (i) is complete. 
Suppose $\&$ contains an elementary subgroup of order $3^{3}$. Since $\mathfrak{Q} \triangleleft \mathfrak{S}, \mathfrak{L}$ contains a $S_{3}$-subgroup $\mathfrak{P}$ which is permutable with $\mathfrak{T}$. Thus, $\bar{X}_{1}$ normalizes $\bar{P}$. By Lemma $13.49, \bar{X}_{1}$ centralizes a $S_{3^{\prime}}$-subgroup of $\overline{\mathfrak{\Omega}}$, so $\bar{X}_{1}$ does not centralize $\overline{\mathfrak{P}}$. Hence, $\mathfrak{P}$ contains an elementary subgroup $₹$ of order $3^{3}$ such that $\bar{X}_{1}$ normalizes $\bar{F}$ and $\bar{X}_{1}$ does not centralize $\bar{F}$. Let $\mathbb{1}_{1}=\mathscr{\Omega} \leftarrow\left\langle X_{1}\right\rangle$. We assume without loss of generality that $X_{1}$ inverts $Q \in \mathfrak{F}^{*}$. Let $\mathfrak{S}^{*}=[\mathfrak{S},\langle Q\rangle]$. Thus, $\left\langle\mathfrak{F}, X_{1}\right\rangle$ normalizes $\mathfrak{S C}^{*}$. By Lemma 13.49, $C_{\mathfrak{E}}\left(\mathfrak{S}^{*}\right)=\mathfrak{F}_{0} \neq 1$. Since $X_{0} \in \mathfrak{S E}^{*}$, we

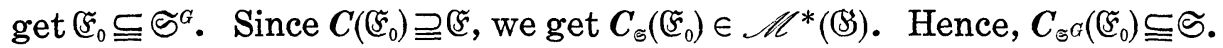
Hence, $S_{3}$-subgroups of $\subseteq \cap \mathfrak{S}^{G}$ are non cyclic, against Theorem 13.1 (b)(ii). The proof is complete.

Lemma 13.51. Assume the hypotheses of Lemma 13.50 hold. Then the following hold:

(a) $\left[\overline{\mathfrak{L}}_{0}, \overline{\mathfrak{X}}_{1}\right]$ is of order 3 .

(b) $\Re / \Re_{0}$ is elementary of order $2^{1+e}$, where $e=0$ or 1 .

(c) $\mathfrak{S}$ contains a subgroup $\mathfrak{S E}^{*}$ such that $\mathfrak{S}^{*}$ is the central product of 2 quaternion groups, $\mathfrak{S}=\mathfrak{S}_{\mathfrak{E}}^{*} C_{\mathfrak{F}}\left(\mathfrak{S E}^{*}\right)$, and $\left|\boldsymbol{C}_{\mathfrak{F}}\left(\mathfrak{S}^{*}\right)\right| \leqq 2^{4}$.

(d) $\mathfrak{S C}_{\mathfrak{S}^{*}}$ is elementary and $\left\langle\mathfrak{B}, \mathfrak{S} \cap \mathfrak{S}^{G}\right\rangle$ is of index at most $2^{3}$ in $\mathfrak{S}$.

Proof. The difficult step is to show that if $\mathfrak{Q}$ is a non abelian subgroup of $\boldsymbol{O}_{2,2^{\prime}}(\mathfrak{S})$ of order $3^{3}$ and exponent 3 , and $\mathfrak{X}_{1}$ normalizes $\Re \Omega$, then $\left[\mathfrak{Q}, \mathfrak{X}_{1}\right] \leqq \Re$. Suppose this assertion is false. Let $\widehat{\mathfrak{Q}}=\{Q \mid Q \in \mathfrak{\Omega}$, $\bar{X}_{1}$ inverts $\left.\Re Q\right\}$, and let $\mathfrak{\Omega}_{0}=\left\{Q \mid Q \in \mathfrak{\Omega},\left[X_{1}, Q\right] \in \mathfrak{R}\right\}$. Thus, $\widehat{\mathfrak{\Omega}} \cap \mathfrak{\Omega}_{0}=\varnothing$. Since $\mathfrak{\Omega}$ is non abelian, $\mathfrak{\Omega}_{0} \neq 1$. Since $\left[\mathfrak{Q}, X_{1}\right] \nsubseteq \Re, \mathfrak{\Omega}_{0} \subset \mathfrak{\Omega}$, and so | $\mathfrak{O}_{1}=3$. If $\mathfrak{\Omega}_{0} \neq \mathfrak{\Omega}^{\prime}$, then $\mathfrak{\Omega}^{*}=\left[\mathfrak{Q}, X_{1}\right] \Re \cap \Omega$ is elementary of order $3^{2}$ and $X_{1}$ inverts $\mathfrak{Q}^{*} \mathfrak{R} / \mathfrak{R}$, against Lemma 13.49 (i). Hence, $\mathfrak{\Omega}_{0}=\mathfrak{\Omega}^{\prime}$, and $X_{1}$ inverts $\Re \cong / \Re \Omega \prime$.

Let $\tilde{\mathfrak{S}}=[\mathfrak{Y}, \mathfrak{Q}]=[\Re, \mathfrak{\Omega}]$, the second equality holding since $|\mathfrak{R}: \mathfrak{S}| \leqq 2$.

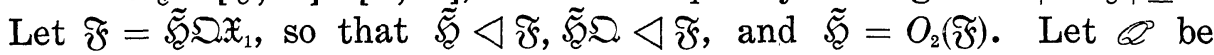
the set of subgroups of $\mathfrak{F}$ of order 3 which are inverted by $X_{1}$. We will show that $\mathscr{Q}$ contains elements $\mathfrak{\Omega}^{1}, \Omega^{2}$ such that $\left\langle\mathfrak{Q}^{1}, \Omega^{2}\right\rangle$ is a $S_{3}$-subgroup of $\mathfrak{F}$. Suppose this too is false.

For each $\mathfrak{A} \in \mathbb{Q}$, let $\tilde{\mathfrak{S}}(\mathfrak{U})=[\tilde{\mathfrak{F}}, \mathfrak{A}]$. By Lemma $13.49, \tilde{\mathfrak{H}}(\mathfrak{N})$ is the central product of 2 quaternion groups. Since $X_{1}$ inverts $\tilde{\mathscr{S}} \cong / \tilde{\mathcal{S}} \mathfrak{Q}$, it follows that if $\mathfrak{A}_{1}, \mathfrak{N}_{2}$ are elements of $Q^{2}$ whose images in $\mathfrak{F} / \tilde{\mathcal{E}}$ are distinct, then $\left\langle\mathfrak{A}_{1}, \mathfrak{A}_{2}\right\rangle$ contains a $S_{3}$-subgroup of $\mathfrak{F}$. Hence,

$$
\left\langle\tilde{\mathfrak{S}}\left(\mathfrak{A}_{1}\right), \tilde{\mathfrak{S}}\left(\mathfrak{A}_{2}\right)\right\rangle=\tilde{\mathfrak{S}},
$$

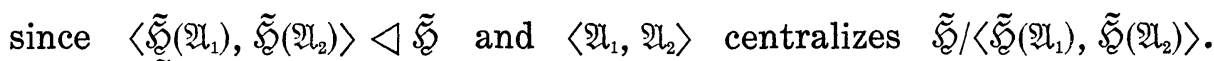
Hence, $\tilde{\mathfrak{H}}$ is the product of 4 or fewer quaternion groups. On the other hand, $\tilde{\mathfrak{F}}\left(\mathfrak{A}_{i}\right)$ contains $\left[\tilde{\mathfrak{F}}, \mathfrak{X}_{1}\right]$, by Lemma 13.49 , and $\left[\tilde{\mathfrak{F}}, \mathfrak{X}_{1}\right]$ is 
elementary of order $2^{3}$. Hence, $\left|\tilde{\mathfrak{F}}\left(\mathfrak{R}_{1}\right) \cap \tilde{\mathfrak{F}}\left(\mathfrak{A}_{2}\right)\right| \geqq 2^{3}$, so that $|\tilde{\mathfrak{F}}| \leqq$ $2^{7}=2^{5+5-3}$. Since $\mathfrak{Z}$ is faithfully represented on $\tilde{\mathfrak{F}} / D(\tilde{\mathcal{F}})$, it follows that $\tilde{\mathfrak{F}}$ is extra special of order $2^{7}$.

Choose a fixed element $\mathfrak{A}$ of $\mathbb{Q}$ and choose notation so that $\mathfrak{Q} \subset \mathfrak{\Omega}$. Let $\tilde{\mathfrak{F}}=\tilde{\mathfrak{S}}(\mathfrak{Z}) \cdot \mathfrak{B}$, where $\mathfrak{B}=\tilde{\mathfrak{F}} \cap \boldsymbol{C}(\tilde{\mathfrak{F}}(\mathfrak{A}))$. Thus, $\mathfrak{B}$ is non abelian of order 8. Let $\mathfrak{D}=N_{\mho_{0}}(\mathfrak{D})$, where $\mathfrak{\mho}_{0}=\tilde{\mathfrak{F}} \mathfrak{X}$. Thus, $|\mathfrak{D}|=4$ and $\tilde{\mathfrak{F}} \cap \mathfrak{D}=\tilde{\mathfrak{F}^{\prime}}$ is of order 2. Choose $D \in \mathfrak{D}-\tilde{\tilde{\mathcal{F}}}$. Then $D=X_{1} H$ with $H \in \tilde{\tilde{F}}$. Hence,

$$
H^{-1} X_{1} \mathfrak{A} X_{1} H=\mathfrak{A}^{H} \subseteq \mathfrak{D},
$$

so that $H \in \mathfrak{B}$. Now $C_{\mathfrak{Q}}(\mathfrak{U})=\mathfrak{A} \times \mathfrak{Q}^{\prime}$, so $\mathfrak{Q}^{\prime}$ normalizes $\mathfrak{B}=\tilde{\mathfrak{F}} \cap \boldsymbol{C}(\mathfrak{2})$. Since $\mathfrak{Q}^{\prime}$ has no non trivial fixed points on $\tilde{\mathfrak{F}} / \boldsymbol{D}(\tilde{\mathfrak{F}})$, it follows that $\mathfrak{B}$ is a quaternion group. By Lemma $13.49, X_{1}$ centralizes $\mathfrak{B}$. Let $Q$ be a generator for $\mathfrak{Q}^{\prime}$. We will show that $\left[X_{1}, Q\right] \in \mathfrak{B}$. Namely, $\tilde{\mathfrak{F}} \mathfrak{Q}^{\prime} / \tilde{\mathfrak{F}}$ is a central factor of $\mathfrak{F}$, so $D$ centralizes $Q$, and $X_{1} Q X_{1}=Q H_{1}$ with $H_{1} \in \tilde{\mathcal{F}}$. Since $D=X_{1} H$, we get $Q=H^{-1} Q H_{1} H$, or equivalently, $H_{1}=Q^{-1} H Q H^{-1}$. Since $H \in \mathfrak{B}$, and $Q$ normalizes $\mathfrak{B}$, we get $H_{1} \in \mathfrak{B}$. Hence, $\tilde{\widetilde{F}}=\mathfrak{B Q}^{\prime}\left\langle X_{1}\right\rangle$ is a group. Thus,

$$
\mathfrak{B}\left\langle X_{1}\right\rangle=\mathfrak{B} \times \mathfrak{X}_{1}=\mathfrak{B} \cdot C_{\widetilde{\mathfrak{F}}}(\mathfrak{B}) \triangleleft \tilde{\mathfrak{F}} .
$$

Since $\mathfrak{B}^{\prime} \times\left\langle X_{1}\right\rangle=\Omega_{1}\left(\mathfrak{B}\left\langle X_{1}\right\rangle\right)$, it follows that $Q$ centralizes $X_{1}$. Hence, $Q$ centralizes $H$, so $H \in \tilde{\mathscr{F}^{\prime}}$, which gives $X_{1} \in \mathfrak{D}$. We have therefore succeeded in showing that $X_{1}$ lies in a systemizer of $\mathfrak{F}$.

Since $\mathfrak{Q}^{\prime} \cong \boldsymbol{C}\left(X_{1}\right)$, it follows that $\mathfrak{\Omega}^{\prime}$ normalizes $\mathfrak{B}_{0}^{G}$. Hence, $\mathfrak{\Omega}^{\prime}$ centralizes $\mathfrak{B}_{0}^{\sigma}$ since $\mathfrak{\Omega}^{\prime}$ stabilizes $\mathfrak{X} \supset \mathfrak{X}_{1} \supset 1$. This is impossible, since $X_{0}$ is a non central element of $\tilde{\mathscr{F}}$ and $\mathfrak{S} \cap \boldsymbol{C}\left(\mathfrak{\Omega}^{\prime}\right)=\boldsymbol{Z}(\tilde{\mathfrak{F}})$. This establishes the first reduction of the proof.

We turn to (a). Let $\Omega_{1}=\left[\Omega_{0}, \mathfrak{X}_{1}\right] \Omega$ and suppose $\left|\bar{\Omega}_{1}\right| \neq 3$. already know that $\left|\overline{\mathbb{L}}_{1}\right|$ is a power of 3 , so suppose $\left|\overline{\mathbb{L}}_{1}\right|>3$. By Lemma 13.49, $\overline{\mathfrak{\Omega}}_{1}$ is non abelian. Let $\bar{\Omega}_{2}$ be a subgroup of $\bar{\Omega}_{1}$ such that $\overline{\mathfrak{\Omega}}_{2} \supset$ $C_{\bar{s}_{1}}\left(X_{1}\right),\left|\overline{\mathfrak{\Omega}}_{2}: C_{\overline{\mathfrak{s}}_{1}}\left(X_{1}\right)\right|=3^{2}$. Let $\overline{\mathfrak{\Omega}}_{3}=\left[\overline{\mathfrak{\Omega}}_{2}, \overline{\mathfrak{X}}_{1}\right]$, so that $\left|\overline{\mathfrak{\Omega}}_{3}: D\left(\overline{\mathfrak{\Omega}}_{3}\right)\right|=3^{2}$, and $X_{1}$ centralizes $\boldsymbol{D}\left(\overline{\mathfrak{\Omega}}_{3}\right)$. By Lemma $13.49, \bar{\Omega}_{3}$ is non abelian, so $\bar{\Omega}_{3}$ is of exponent 3 and order $3^{3}$, against the first part of the proof. This establishes (a).

Since $\boldsymbol{D}(\mathfrak{R}) \subseteq \mathfrak{X}=\mathfrak{B}_{0}^{G}$, it follows that $\boldsymbol{D}(\overline{\mathfrak{R}}) \subseteq \overline{\mathfrak{X}}_{1}$. By (a), $\overline{\mathfrak{X}}_{1}$ is not a square in $\bar{\Re}$, so $D(\bar{\Re})=1$. By Lemma 13.50 , (b) follows.

We let $\mathfrak{Q}=\langle Q\rangle$ be a subgroup of $\mathfrak{\Omega}_{0}$ of order 3 inverted by $X_{1}$, and we define $\mathfrak{S}^{*}=\left[\mathfrak{S}_{\mathfrak{C}}, \mathfrak{\Omega}\right]$. By Lemma $13.49, \mathfrak{S}=\mathfrak{S}^{*} \boldsymbol{C}_{\mathfrak{F}}\left(\mathfrak{S}^{*}\right)$, so it suffices to show that $\left|\boldsymbol{C}_{\mathfrak{5}}\left(\mathfrak{S C}^{*}\right)\right| \leqq 2^{4}$. Let $\mathfrak{S}_{1}^{*}=\boldsymbol{C}_{\mathfrak{5}}\left(\mathfrak{S C}^{*}\right)$. Thus, $\boldsymbol{Z}\left(\mathfrak{S}_{1}^{*}\right)=$ $\mathfrak{B}=\boldsymbol{Z}(\mathfrak{S})$ is of order 4 . By $(\mathrm{b})$, we have $\left|\mathfrak{R}_{0}\right|=\left|\mathfrak{S}^{G}\right| \cdot 2^{-2-e}=\left|\mathfrak{S}_{2}\right| \cdot 2^{-2-e}$. Let $\Re_{1}=C_{\Re_{0}}(\mathfrak{B})=\Re_{0} \cap \mathfrak{S}_{\mathcal{E}}$, so that $\left|\Re_{1}\right| \geqq\left|\mathfrak{S}_{2}\right| \cdot 2^{-3-e}$. Now $\boldsymbol{D}\left(\Re_{1}\right) \subseteq \mathfrak{B} \cap$ $\mathfrak{B}^{G}=1$, and $\mathfrak{X}_{1}$ centralizes $\mathfrak{R}_{1}$, while $\left\langle\mathfrak{R}_{1}, \mathfrak{X}_{1}\right\rangle=\mathfrak{R}_{1} \times \mathfrak{X}_{1}$. Hence, $\mathfrak{S}^{G}$ contains an elementary subgroup of index at most 8 , so $\mathfrak{S}$ contains an elementary subgroup $\left(\mathfrak{F}\right.$ with $\left|\mathfrak{S}_{\mathcal{E}}: \mathfrak{F}\right| \leqq 8, \mathfrak{B} \cong \mathbb{F}$. Since $\mathfrak{F}^{*}$ is extra 
special of order $2^{5}$, we get that $\mathfrak{S}^{*} \mathfrak{F}$ is of index at most 2 in $\mathfrak{S}_{\text {. }}$. Let $3=\mathfrak{S}^{*}$, so that $\mathfrak{S}^{*} \mathfrak{S} / 3$ is an elementary subgroup of $\mathfrak{S} / 3$ of index at most 2. First, suppose $\mathfrak{S}^{*} \mathfrak{F}=\mathfrak{K}$. In this case, $\boldsymbol{D}(\mathfrak{F})=3$, so that

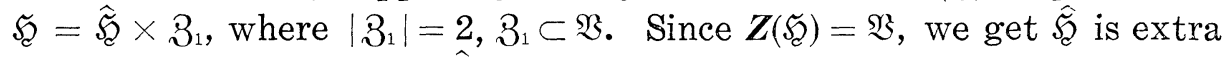
special, so the width of $\hat{\mathcal{F}}$ is at most 3 , since $\mathfrak{F}$ exists. The proof is complete in this case. We may assume that $\mathfrak{F}^{*}[\mathfrak{F} \subset \mathfrak{F}$. This implies that $\left|\mathfrak{S}^{*} \cap \mathfrak{F}\right|=8$, since $\left|\mathfrak{S C}^{*}\right|=2^{5}$ and $\mid \mathfrak{F}$ : $\mathfrak{F} \mid \leqq 8$. Hence, $\mathfrak{S C}^{*} \cap \mathfrak{F} \in$ $\mathscr{S} \mathscr{C} \mathscr{N}^{-}\left(\mathfrak{S}_{2}^{*}\right)$. Hence, $\mathfrak{F} \subseteq\left(\mathfrak{S}^{*} \cap(\mathfrak{F}) \mathfrak{S}_{1}^{*}\right.$, so that $\left|\mathfrak{S}_{1}^{*}: \mathfrak{S}_{1}^{*} \cap \mathfrak{F}\right| \leqq 2$; the inequality holding since $\mid \mathfrak{F}_{2}:\left(\mathfrak{S}_{\mathcal{C}}^{*} \cap(\mathbb{E}) \mathfrak{K}_{1}^{*} \mid=4\right.$. Suppose $\left|\mathfrak{S}_{1}^{*}\right|=2^{b}>2^{4}$. Since $Z\left(\mathfrak{S}_{1}^{*}\right)=\mathfrak{B}$, and since $\mathfrak{S}_{1}^{*}$ stabilizes the chain $\mathfrak{S}_{1}^{*} \cap \mathfrak{F} \supset \mathfrak{B} \supset 1$, and since $\left|\mathfrak{S}_{1}^{*}: \mathfrak{S}_{1}^{*} \cap \mathfrak{F}\right|=2$, we get that $b=5$, and that $\mathfrak{S}_{1}^{*}$ contains exactly 1 elementary subgroup of order $2^{4}$, namely, $\mathfrak{S}_{1}^{*} \cap \mathfrak{5}$. Hence, $|\mathfrak{F}|=2^{6}$, every elementary subgroup of $\mathfrak{K}$ of order $2^{6}$ is contained in

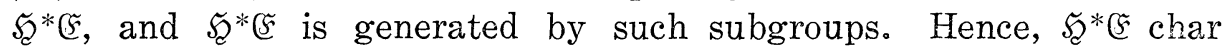
$\mathfrak{S}$, against $m\left(\mathbb{Z}\left(\mathfrak{S}_{\mathcal{C}}^{*}(\mathfrak{F})\right)=4\right.$. The proof is complete.

Lemma 13.52. The hypotheses of Lemma 13.50 are not satisfied.

Proof. Suppose false. Let $\mathfrak{\Omega}=\langle Q\rangle$ be a subgroup of $\mathfrak{\Omega}_{0}$ of order 3 inverted by $X_{1}$, and let $\mathfrak{F}$ be a $S_{2^{2}}$-subgroup of $\mathcal{L}_{0}$ which contains న. By Lemma $13.51,\left[\overline{\mathfrak{L}}_{0}, \overline{\mathfrak{X}}_{1}\right]=\overline{\mathfrak{D}}$, and so $\overline{\mathfrak{D}}$ is a direct factor of $\overline{\mathfrak{P}}=\bar{\Omega}_{0}$. Hence, $\mathfrak{P}=\mathfrak{Q} \times \mathfrak{P}_{0}$ for a suitable subgroup $\mathfrak{P}_{0}$ of $\mathfrak{P}$. Since $\mathfrak{P}$ has no elementary subgroup of order $3^{3}$, a $S_{3}$-subgroup of $\mathfrak{F}_{0}$ is cyclic.

Let $\mathfrak{S}^{*}=[\mathfrak{K}, \mathfrak{D}]$, and let $\mathfrak{B}$ be the $S_{3^{\prime}}$-subgroup of $\mathfrak{B}$. Since Aut $\left(\mathfrak{S}^{*}\right)$ is a 2, 3-group, $\mathfrak{B}$ centralizes $\mathfrak{S}^{*}$. Since $\left|\boldsymbol{C}_{\mathfrak{F}}\left(\mathfrak{S}_{\mathfrak{C}}^{*}\right)\right| \leqq 2^{4}$ and since $\mathfrak{B} \subseteq C_{\mathfrak{p}}\left(\mathfrak{S}^{*}\right)$, it follows that $\mathfrak{B}$ centralizes $C_{\mathfrak{S}}\left(\mathfrak{S}^{*}\right)$. Hence, $\mathfrak{B}$ centralizes $\mathfrak{S}=\mathfrak{S}^{*} C_{\mathfrak{S}}\left(\mathfrak{S}^{*}\right)$. Since $\left|\Re: \mathfrak{F}_{\mathfrak{C}}\right| \leqq 2$, $\mathfrak{B}$ centralizes $\Re$, so $\mathfrak{B}=1$, as $\boldsymbol{O}_{2},(\mathfrak{S})=1$.

Since a $S_{3}$-subgroup of Aut $\left(\mathfrak{F}^{*}\right)$ is elementary of order $\left.3^{2}, \sigma^{1}: \mathfrak{S}^{*}\right)$ centralizes $\mathfrak{S}^{*}$ and since $\left|\boldsymbol{C}_{\mathfrak{S}}\left(\mathfrak{S}^{*}\right)\right| \leqq 2^{4}, \mathscr{O}^{1}(\mathfrak{P})$ also centralizes $\boldsymbol{C}_{\mathfrak{5}}\left(\mathfrak{S}^{*}\right)$, hence centralizes $\mathfrak{S}$ and $\mathscr{R}$, so that $\tilde{O}^{1}(\mathfrak{P})=1$. Hence, $\mathfrak{P}$ is elementary of order 3 or $3^{2}$.

First, suppose $|\mathfrak{P}|=3$. By definition of $\mathbb{\Omega}_{0}$ and by 0.3 .3 , we get $\subseteq=\mathfrak{Z}_{0} \mathfrak{X}_{1}$. Let $\Re_{1}=\Re_{0} \cap \mathfrak{S}_{\mathfrak{Z}}$ so that $\left|\Re_{0}: \Re_{1}\right| \leqq 2$. Since $\boldsymbol{D}\left(\Re_{1}\right) \subseteq \mathfrak{X} \cap$ $\mathfrak{B}=1$, it follows that $\left\langle\mathfrak{R}_{1}, \mathfrak{X}_{1}\right\rangle$ is an elementary subgroup of $\mathfrak{F}^{G}$ of index $2^{1+a}$, where $2^{a}=\left|\Re_{0}: \Re_{1}\right|$. Since $\mathfrak{S}^{*}$ is extra special of order $2^{5}$, we get $a=1$. Thus, $\mathfrak{K}$ has an elementary subgroup $\sqrt{5}$ of index 4 , so $\mathfrak{S}_{\mathfrak{C}}=\mathfrak{S}^{*} \mathfrak{F}_{\text {. }}$. This implies that $C_{\mathfrak{f}}\left(\mathfrak{S}^{*}\right)$ is elementary, so $C_{\mathfrak{F}}\left(\mathfrak{S}^{*}\right)=\mathfrak{B}$, since $\mathfrak{B}=\mathbb{Z}\left(\boldsymbol{C}_{\mathfrak{F}}\left(\mathfrak{S}^{*}\right)\right)$. Let $\mathfrak{W}=\boldsymbol{C}_{\mathfrak{\Omega}}(\mathfrak{Q})$, so that $\mathfrak{W}$ is dihedral of order 8 and $\mathfrak{R}=\mathfrak{S} \mathfrak{M}, \mathfrak{S} \cap \mathfrak{S}=\mathfrak{B}$. Since $\mathfrak{W}$ admits $X_{1}$ and $X_{1}$ centralizes $\mathfrak{B}$, it follows that $\left[\mathfrak{W}, \mathfrak{X}_{1}\right] \subseteq \boldsymbol{Z}(\mathfrak{W})=\mathfrak{S}^{*}$. Choose $W \in \mathfrak{W}-\mathfrak{B}$ with $W^{2}=1$. If $W$ induces an inner automorphism of $\mathfrak{S}^{*}$, then $\mathfrak{B}$ cen-

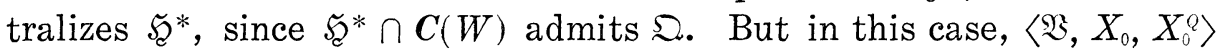


is a normal elementary subgroup of $\subseteq$ of order 16 . Hence, $W$ does not induce an inner automorphism of $\mathfrak{S}^{*}$. Let

$$
\mathfrak{S}_{0}^{*}=\left\{H \mid[W, H] \in \mathfrak{S}^{*^{\prime}}, H \in \mathfrak{S}^{*}\right\} \text {. }
$$

Since $\mathfrak{R}=\mathfrak{S}_{\mathfrak{B}} \cdot \Re_{0}$, it follows that $X_{0} \in \mathfrak{S}_{0}^{*}$. Thus, $\mathfrak{S}_{0}^{*}$ is not a quaternion group, and $\mathfrak{S}_{0}^{*}$ admits $\mathfrak{\Omega}$. The only possibility is that $\mathfrak{S}_{0}^{*}$ is elementary of order 8. Thus, $\mathfrak{S}_{0}^{*} \triangleleft \mathfrak{S}$, again a contradiction.

It remains to treat the case $|\mathfrak{P}|=3^{2}$. We first show that $[\mathfrak{K}, \mathfrak{F}]=$ $\mathfrak{S}^{*}$. Suppose false. Set $\mathfrak{S}_{1}^{*}=C_{\mathfrak{F}}\left(\mathfrak{S E}^{*}\right), \mathfrak{S}_{2}^{*}=\left[\mathfrak{S}_{1}^{*}, \mathfrak{B}\right]$, so that $\mathfrak{S}_{2}^{*} \neq 1$, $\mathfrak{S}_{2}^{*} \subseteq \mathfrak{S}_{1}^{*}$. Since $[\mathfrak{B}, \mathfrak{P}]=1, \mathfrak{B} \subseteq \mathfrak{S}_{1}^{*},\left|\mathfrak{S}_{1}^{*}: \mathfrak{B}\right| \leqq 4$, and $\boldsymbol{Z}\left(\mathfrak{S}_{1}^{*}\right)=\mathfrak{B}$, it follows that $\mathfrak{S}_{2}^{*}$ is a quaternion group. If $\mathfrak{S}=\mathfrak{S}_{2}^{*} \times \mathfrak{S}^{*}$, then $\Omega_{1}(\mathfrak{S})=$ $\mathfrak{S}_{2}^{* \prime} \times \mathfrak{K}^{*}$, so that $\mathfrak{S}$ has no elementary subgroup of index 8. If $\mathfrak{S}_{\mathrm{Z}} \neq \mathfrak{S}_{2}^{*} \times \mathfrak{S}^{*}$, then $\mathfrak{S}_{2}^{*} \mathfrak{S}^{*} \subset \mathfrak{S}$, so that $\mathfrak{S E}_{2}^{*} \mathfrak{H}_{2}^{*}$ is the central product of 3 quaternion groups, and $\mathfrak{S}_{2}=\mathfrak{S}_{2}^{*} \mathfrak{S}_{2}^{*} \times 3$, for some subgroup 3 of $\mathfrak{B}$ of order 2. Again, we see that $\mathfrak{F}$ has no elementary subgroup of index 8. Thus, Lemma 13.51 (d) yields a contradiction.

Now $\mathfrak{S}=\Re \cdot N_{\mathfrak{S}}(\mathfrak{P})$, and since $\mathfrak{S}^{*}=[\mathfrak{S}, \mathfrak{P}]=[\mathfrak{R}, \mathfrak{P}]$, we get $\mathfrak{S}^{*} \triangleleft \mathfrak{S}$. Since $\mathfrak{S}_{\mathcal{E}}^{*} \mathfrak{P}$ permutes transitively the non central involutions of $\mathfrak{S}^{*}$, it follows that $\left\langle\mathfrak{S}^{* \prime}, X_{0}\right\rangle$ is normal in some $S_{2}$-subgroup $\mathfrak{I}^{*}$ of $\mathfrak{S}$, with $\mathfrak{X} \subset \mathfrak{I}^{*}$. Thus, $\mathfrak{T}^{*}=\mathfrak{S}^{*} \cdot C_{\mathfrak{x}^{*}}\left(\mathfrak{X}_{0}\right)$. Thus, $\left[\mathfrak{T}^{*}, \mathfrak{X}_{1}\right] \subseteq \mathfrak{S}^{*}$, since $\left[C_{\mathfrak{x}^{*}}\left(\mathfrak{X}_{0}\right), \mathfrak{X}_{1} \subseteq\right.$ $\mathfrak{X}_{0}$, due to $C_{\mathfrak{x}^{*}}\left(\mathfrak{X}_{0}\right) \subseteq \subseteq^{\sigma}$. Thus, $\mathfrak{X}_{1}$ is a central subgroup of $\mathfrak{I}^{*}$ of order 2. Hence, $\sqrt[\mathcal{D}]{\text { admits }} \overline{\mathfrak{T}}^{*}$. This implies that $\overline{\mathfrak{T}}^{*}$ is elementary of order $2^{b}$ with $b \leqq 2$, which in turn implies that $\subseteq=\mathfrak{T}^{*} \mathfrak{P}$. Hence, $\boldsymbol{D}\left(\mathfrak{T}^{*}\right) \subseteq \Re$, and since $\boldsymbol{D}\left(\mathfrak{I}^{*}\right)$ obviously centralizes $\mathfrak{B}$, we even get $\boldsymbol{D}\left(\mathfrak{I}^{*}\right) \subseteq \mathfrak{S}$.

We next show that $\mathfrak{B}$ is weakly closed in $\mathfrak{F}$. For suppose $Y \in$ (S) - $\subseteq$ and $\mathfrak{B}^{Y} \subseteq \mathfrak{S}$. Let $A_{1}, A_{2}, A_{3}$ be the involutions of $\mathfrak{B}^{Y}$. Thus, $A_{i}=A_{i 1} A_{i 2}$, where $A_{i 1} \in \mathfrak{S}^{*}, A_{i 2} \in C_{H}\left(\mathfrak{S}^{*}\right)$. Since $\left[C_{\mathfrak{S}}\left(A_{i}\right), \mathfrak{B}^{Y}\right] \subseteq \mathfrak{B}^{Y} \cap \mathfrak{B}=1$, we get $C_{\mathfrak{5}}\left(A_{i}\right)=C_{\mathfrak{g}}\left(\mathfrak{B}^{Y}\right), i=1,2,3$. Since $\mathfrak{S}^{*}$ is extra special, it follows that $\mathfrak{S}^{*} \subseteq C_{\mathscr{G}}\left(\mathfrak{B}^{Y}\right)$, that is, $\mathfrak{B}^{Y} \subseteq C_{\mathfrak{5}}\left(\mathfrak{S}^{*}\right)$. Since $\left|\boldsymbol{C}_{\mathfrak{F}}\left(\mathfrak{S}_{\mathcal{C}}^{*}\right)\right| \leqq 16$, we $\boldsymbol{C}_{\mathfrak{S}}\left(\mathfrak{S}^{*}\right)=\mathfrak{B} \times \mathfrak{B}^{Y}$, against $\boldsymbol{Z}\left(\boldsymbol{C}_{\mathfrak{\Re}}\left(\mathfrak{F}^{*}\right)\right)=\mathfrak{B}$. So $\mathfrak{B}$ is weakly closed in $\mathfrak{\mathcal { E }}$.

Let $\tilde{\mathfrak{I}}$ be a $S_{2}$-subgroup of $\mathfrak{S}^{G}$ which contains a $S_{2}$-subgroup of $\boldsymbol{C}_{\mathfrak{S}}\left(X_{0}\right)$. Thus, $\mathfrak{B} \cong \tilde{\mathfrak{I}}$. If $\mathfrak{B} \subseteq \boldsymbol{D}(\tilde{\mathfrak{I}})$, we get $\mathfrak{B} \cong \mathfrak{S}^{G}$, since $\boldsymbol{D}(\tilde{\mathfrak{I}}) \subseteq \mathfrak{S}^{G}$. Hence, $\mathfrak{B}=\mathfrak{B}^{G}$, since $\mathfrak{B}^{G}$ is weakly closed in $\mathfrak{S}^{G}$. This is absurd, so $\mathfrak{B} \nsubseteq \boldsymbol{D}(\tilde{\mathfrak{T}})$. In particular, $\mathfrak{B} \nsubseteq \boldsymbol{D}\left(\boldsymbol{C}_{\mathfrak{p}}\left(\mathfrak{X}_{0}\right)\right)$. Now $\boldsymbol{C}_{\mathfrak{F}^{*}}\left(\mathfrak{X}_{0}\right)$ is the direct product of $\left\langle X_{0}\right\rangle$ and a dihedral group of order 8. Hence, $\mathfrak{S}_{\mathcal{C}^{* \prime}} \subseteq C_{\mathfrak{S}^{*}}\left(X_{0}\right)^{\prime}$. This implies that $\mathfrak{S}^{* \prime}=\boldsymbol{D}\left(\boldsymbol{C}_{\mathfrak{\mathfrak { S }}}\left(X_{0}\right)\right)$. Let $\mathfrak{S}_{1}^{*}=\boldsymbol{C}_{\mathfrak{S}}\left(\mathfrak{S}^{*}\right) \subset \boldsymbol{C}_{\mathfrak{5}}\left(X_{0}\right)$. Thus,

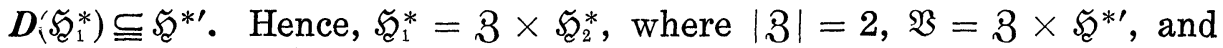
$D\left(\mathfrak{S}_{2}^{*}\right) \subseteq \mathfrak{S}_{2}^{* \prime}$. Hence, $\mathfrak{S}_{2}=\mathfrak{S}_{2}^{*} \mathfrak{S}_{2}^{*} \times 3$. Since $\boldsymbol{Z}(\mathfrak{S})=\mathfrak{B}, \mathfrak{S}_{2}^{*} \mathfrak{S}_{2}^{*}$ is extra special of width 2 or 3 .

Since $\mathfrak{S}^{*}=[\mathfrak{F}, \mathfrak{P}]$, we get $[\Re, \mathfrak{P}]=[\Re, \mathfrak{Q}]$. Let $\mathfrak{I}_{1}=N_{\mathfrak{x}}(\mathfrak{P})$, so that $\mathfrak{S}=\mathfrak{S}_{\mathfrak{C}}^{*} \mathfrak{P \mathfrak { I } _ { 1 }}$. We can thus choose $S_{1}$ in $\mathfrak{I}_{1}$ such that $X_{1}=H^{*} S_{1}$ with $H^{*} \in \mathfrak{S}^{*}$. Since $\mathfrak{V} \triangleleft N_{\mathfrak{S}}(\mathfrak{P})$, it follows that both $X_{1}$ and $S_{1}$ normalize $\mathfrak{\Omega}$. Hence, $H^{*} \in \mathfrak{S}^{*} \cap N(\mathfrak{\Omega})=\mathfrak{S}^{* \prime}$. Hence, $X_{1}$ normalizes $\mathfrak{P}$. 
Let $\mathfrak{P}=\mathfrak{\Omega} \times \mathfrak{\Omega}_{0}$, where $\mathfrak{\Omega}_{0}=C_{\mathfrak{\beta}}\left(X_{1}\right)$. Thus, $\mathfrak{\Omega}_{0} \subseteq C\left(X_{1}\right) \leqq \mathfrak{B}^{G}$, so $\mathfrak{\Omega}_{0}$ centralizes $\mathfrak{S}^{G}$. Hence, $X_{0} \in C_{\mathfrak{\$}^{*}}\left(\Omega_{0}\right)$. This is impossible, since the centralizer in $\mathfrak{S}^{*}$ of every non identity element of $\mathfrak{P}$ is contained in a quaternion subgroup of $\mathfrak{S}_{\mathfrak{C}}^{*}$. The proof is complete.

\section{LEMMA 13.53. $\mathfrak{I} \in \mathscr{C}^{*}(\mathbb{S})$.}

Proof. Suppose false, and $\mathfrak{I} \subset \mathfrak{N} \in \mathscr{S} \mathscr{O} \mathscr{L}(\mathbb{S}), \mathfrak{N} \subset \mathfrak{S}$. We assume without loss of generality that if $\mathfrak{I} \subseteq \mathfrak{N}_{0} \subset \mathfrak{N}$, then $\mathfrak{N}_{0} \subseteq S_{\text {. Hence, }}$ $\mathfrak{R}=\mathfrak{T} \mathfrak{\Omega}$, where $\mathfrak{D}$ is a $q$-group for some odd prime $q$.

By Lemma $13.44, \mathfrak{B} \subseteq \boldsymbol{O}_{2}(\mathfrak{R})=\mathfrak{N}_{1}$, say. Since $\mathfrak{R}_{1} \subseteq \mathfrak{T} \subseteq \mathfrak{S}$, it follows from Lemma 13.41 that $\mathfrak{B}^{N}$ is abelian. Thus, for each $G$ in $\mathfrak{N}$, the normal closure of $\mathfrak{B}_{0}^{G}$ in $\mathfrak{T}$ is abelian.

By minimality of $\mathfrak{N}, \mathfrak{N}_{1} \mathfrak{\Omega} \neg \mathfrak{N}$, and $\mathfrak{N}_{1} \mathfrak{N} / \mathfrak{N}_{1} \boldsymbol{D}(\mathfrak{Q})$ is a chief factor of $\mathfrak{R}$. Suppose $\mathfrak{S}_{\mathfrak{L}} \subseteq \mathfrak{N}_{1}$. By Lemma $13.45(\mathrm{~b})$, we get $\boldsymbol{C}(\mathfrak{S})=Z(\mathfrak{K})=\mathfrak{B}$. Hence, $\boldsymbol{Z}\left(\mathfrak{N}_{1}\right) \subseteq \mathfrak{B}$. By Lemma $13.40, \mathfrak{B}$ is a $\mathrm{T}$. I. set in $\mathbb{S}$, so that $\mathfrak{R} \subseteq \mathfrak{S}$. This is not the case, and so $\mathfrak{S}_{\mathbb{2}} \nsubseteq \mathfrak{R}_{1}$. Since $\mathfrak{S} \triangleleft \mathfrak{I}$, we get $\mathfrak{R}_{1} \mathfrak{D}=\mathfrak{R}_{1}[\mathfrak{Q}, \mathfrak{S}]$, and since $\boldsymbol{D}(\mathfrak{\Omega}) \subseteq \mathfrak{S}$, we get $[\boldsymbol{D}(\mathfrak{\Omega}), \mathfrak{K}] \subseteq \mathfrak{R}_{1} \boldsymbol{D}(\mathfrak{2}) \cap \mathfrak{K} \subseteq$ $\mathfrak{N}_{1}$. By Lemma 0.8.7, $\boldsymbol{D}(\mathfrak{\Omega}) \subseteq Z(\Omega)$, and so $\Omega$ is of exponent $q$.

Since $\mathfrak{S}_{\mathfrak{C}} \cap \mathfrak{R}_{1} \supseteq \mathfrak{B}$, and since $\mathfrak{H} / \mathfrak{B}$ is elementary, it follows that $\mathfrak{S}_{\mathfrak{C}} \mathfrak{N}_{1} / \mathfrak{N}_{1} \cong \mathfrak{S}_{\mathfrak{C}} / \mathfrak{S} \cap \mathfrak{R}_{1} \cong \mathfrak{S} / \mathfrak{B} / \mathfrak{S} \cap \mathfrak{S}_{1} / \mathfrak{B}$ is elementary. Hence, $\mathfrak{D}$ contains a subgroup $\mathfrak{\Omega}_{1}$ of order $q$ such that $\mathfrak{S}$ normalizes $\mathfrak{N}_{1} \mathfrak{\Omega}_{1}$ and $\mathfrak{S}$ does

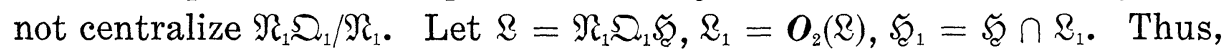
$\left|\mathfrak{S}_{\mathrm{C}}: \mathfrak{F}_{1}\right|=2$, and $\mathfrak{\Omega}_{1}=\langle Q\rangle$, with $Q^{q}=1$.

Let $\mathfrak{W}=\Omega_{1}\left(\boldsymbol{Z}\left(\mathfrak{Q}_{1}\right)\right) \triangleleft \mathfrak{R}$. Then $\mathfrak{W} \supseteqq \Omega_{1}(\boldsymbol{Z}(\mathfrak{T}))$, so that $\mathfrak{W} \cap \mathfrak{B} \neq 1$. Hence, $\mathfrak{\Omega}_{1}$ does not centralize $\mathfrak{W}$. Let $\mathfrak{W}_{1}=C_{\mathfrak{B}}\left(\mathfrak{\Omega}_{1}\right)$. Suppose $\mathfrak{W}_{1} \neq 1$. Then $\mathfrak{W}_{1} \triangleleft \mathfrak{L}$, and so $\mathfrak{W}_{1} \cap \boldsymbol{C}(\mathfrak{S}) \neq 1$. But $Z(\mathfrak{F})=\mathfrak{B}=\boldsymbol{C}(\mathfrak{E})$, against $\mathfrak{\Omega}_{1} \nsubseteq \mathfrak{S}$. Hence, $\boldsymbol{C}_{\mathfrak{B B}}\left(\mathfrak{\sim}_{1}\right)=1$.

Suppose $|\mathfrak{W}|>4$. Let $\mathfrak{\Omega}_{2}=\mathfrak{Q}_{1} \mathfrak{S}, \overline{\mathfrak{L}}_{2}=\mathfrak{\Omega}_{2} / \mathbb{R}_{1} \cong \mathfrak{S} / \mathfrak{S}_{1}$, so that $\left|\overline{\mathfrak{L}}_{2}\right|=2$. Since $\mathfrak{W}$ is a free $F_{2} \overline{\mathfrak{L}}_{2}$-module, we get $\left|\mathfrak{W} \cap \boldsymbol{C}\left(\mathfrak{Q}_{2}\right)\right| \geqq 4$. Hence, $\mathfrak{W} \cap$ $C\left(\Omega_{2}\right)=\Omega_{1}(\mathfrak{B})$ and $|\mathfrak{W}|=2^{4}$. By Lemma 13.46, we get $\mathfrak{W} \subseteq \mathfrak{S}_{\mathrm{C}}$. Thus, $\mathfrak{B}_{0}^{Q} \subseteq \mathfrak{S}_{\mathcal{C}}, \mathfrak{B}^{Q^{-1}} \subseteq \mathfrak{S}$, or equivalently, $\mathfrak{B}_{0}^{Q} \subseteq \mathfrak{S}_{\mathcal{C}}, \mathfrak{B}_{0} \subseteq \mathfrak{S}^{Q}$. This violates Lemma 13.47. Hence, $|\mathfrak{W}|=4, q=3$.

By Lemma 13.47 , either $\mathfrak{B}_{0}^{Q} \nsubseteq \mathfrak{F}$ or $\mathfrak{B}_{0} \nsubseteq \mathfrak{S}_{\mathcal{E}}^{Q}$. Replacing $Q$ by $Q^{-1}$ if necessary, we may assume that $\mathfrak{B}_{0}^{Q} \nsubseteq \mathfrak{F}_{\text {. }}$.

We now let $Q$ play the role of $G$ in Lemma 13.50, and proceed to verify the hypotheses of that lemma. First, $\mathfrak{S}_{1}$ is of index 2 in

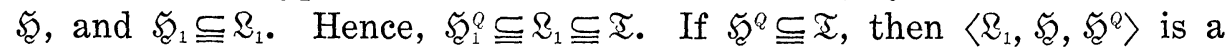
subgroup of $\mathfrak{T}$, against $\left\langle\mathbb{R}_{1}, \mathfrak{F}_{\mathcal{C}}, \mathfrak{S}^{Q}\right\rangle=\mathbb{R}$. Hence, (a) is satisfied. Since $\mathfrak{B} \subseteq \boldsymbol{O}_{2}(\mathfrak{R})$, we get $\mathfrak{B}^{2} \subseteq \boldsymbol{O}_{2}(\mathfrak{N}) \subseteq \mathfrak{T}$, so (b) holds. By our construction, $\mathfrak{B}_{0}^{Q} \cap \mathfrak{S C}_{\mathcal{C}} \subset \mathfrak{B}_{0}^{Q}$. By Lemma 13.46, $\mathfrak{W} \subseteq \mathfrak{S}_{\mathrm{C}}$. Since $C_{\mathfrak{B}}(\mathfrak{S})=\mathfrak{B}_{0} \cap \mathfrak{W}$ is of order 2, we get $\left(\mathfrak{B}_{0} \cap \mathfrak{B}\right)^{Q} \subseteq \mathfrak{S}$, so that (c) holds. By construction, (d) holds, and we have shown that (e) holds, in the first part of the 
proof. Now we have a contradiction with Lemma 13.52, completing the proof.

\section{THEOREM 13.4. Hypothesis 13.3 is not satisfied.}

Proof. Suppose false. By Lemma 13.53, $\mathfrak{I} \in \mathscr{M}^{*}(\mathbb{S})$. Let $\mathfrak{F}$ be the subgroup given in Lemma 13.2. Let $\mathfrak{S}_{2}=\mathfrak{I} \cap \mathfrak{S}_{2}$, and let $\mathfrak{S}_{3}$ be a $S_{3}$-subgroup of $\mathfrak{S}_{\mathcal{E}}$, so that $\mathfrak{S}_{\mathcal{E}}=\mathfrak{S}_{2} \mathscr{S}_{2}$.

Since $\boldsymbol{O}_{3}(\mathfrak{S})=1$, it follows that $Z(\mathfrak{T}) \subseteq Z\left(\boldsymbol{O}_{2}(\mathfrak{S})\right)$. Hence, $\mathfrak{S}_{2}$ contains every element of $\mathscr{U}_{(}(\mathfrak{T})$, so $\mathfrak{S}_{2}$ contains $\mathfrak{B}_{0}$. Let

$$
\mathfrak{A}=V\left(\operatorname{ccl}_{\mathfrak{\Theta}}\left(\mathfrak{B}_{0}\right) ; \mathfrak{S}_{2}\right) \neq 1 \text {. }
$$

Thus, $\mathfrak{A} \pitchfork \mathfrak{S}$, since $\mathfrak{F}_{2}$ is a $S_{2}$-subgroup of $N(\mathfrak{B})$ for every non identity normal subgroup $\mathfrak{B}$ of $\mathfrak{S}$.

We assume without loss of generality that if $\mathfrak{S}_{2} \subseteq \mathfrak{S}_{0} \subset \mathfrak{S}$, then $\mathfrak{S}_{0} \subseteq \mathfrak{S}$. Thus, with $\mathfrak{S}_{1}=\boldsymbol{O}_{2}(\mathfrak{S})$, we get that $\mathfrak{S}_{1} \mathfrak{\Omega} / \mathfrak{S}_{1} \boldsymbol{D}(\mathfrak{Q})$ is a chief factor of $\mathfrak{S}$ and $D(\mathfrak{Q})=\mathfrak{Q} \cap \mathfrak{S}$.

Choose $G$ in $\mathfrak{S}$ such that $\mathfrak{B}_{0}^{G}=\mathfrak{X} \subseteq \mathfrak{S}_{2}, \mathfrak{X} \nsubseteq \mathfrak{S}_{1}$. Let $\mathfrak{\Omega}_{1}$ be a subgroup of $\mathfrak{\Omega}$ minimal subject to (a) $\mathfrak{X}$ normalizes $\mathfrak{S}_{1} \mathfrak{Q}_{1}$, (b) $\left[\mathfrak{Q}_{1}, \mathfrak{X}\right] \nsubseteq \mathfrak{F}_{1}$. Since $\mathfrak{X}$ is elementary, $\mathfrak{\Omega}_{1}$ is cyclic. Let $\mathbb{R}=\mathfrak{K}_{1} \mathfrak{\Omega}_{1} \mathfrak{X}$. Let $\mathfrak{\Omega}_{0}=\boldsymbol{O}_{2}(\mathbb{R})$, $\mathfrak{X}_{0}=\mathfrak{X} \cap \mathfrak{\Omega}_{0}$, so that $\left|\mathfrak{X}_{0}\right|=2$. Let $\mathfrak{Y}=\left[\Omega_{1}\left(Z\left(\mathfrak{Z}_{0}\right)\right), \mathfrak{\Omega}_{1}\right]$. Since $\Omega_{1}(\boldsymbol{Z}(\mathfrak{T})) \cong$ $\Omega_{1}\left(Z\left(\Omega_{0}\right)\right)$, it follows that $\mathfrak{Y} \neq 1$. We assume without loss of generality that $\mathfrak{X}=\mathfrak{X}_{0} \times \mathfrak{X}_{1}, \mathfrak{X}_{i}=\left\langle X_{i}\right\rangle$, and that $X_{1}$ inverts a generator $Q$ of $\mathfrak{\Omega}_{1}$. Since $X_{0}$ centralizes $\mathfrak{Y}$, we get $\left[\mathfrak{V}, X_{1}\right] \subseteq \mathfrak{X}_{0}$. Hence, $|\mathfrak{Y}|=4,\left[\mathfrak{Y}, X_{1}\right]=$ $\mathfrak{X}_{0}$. Hence, $\left[\mathfrak{\Omega}_{0}, X_{1}\right]=\mathfrak{X}_{0}$, since $\Omega_{0} \subseteq C\left(X_{0}\right) \subseteq \varsigma^{a}$. Thus, $Q^{3}$ centralizes $\mathfrak{Y}$, so $Q^{3} \in \mathfrak{S}^{G}$. Since $X_{1}$ inverts, $Q^{3}$, we get $Q^{3}=1$. Hence, $\left[\mathfrak{\Omega}_{0}, \mathfrak{\Omega}_{1}\right]=$ $\left[\mathfrak{S}_{1}, \mathfrak{\Omega}_{1}\right]=\mathfrak{Y}$, a four-group.

We now look again at $\mathfrak{S}_{\text {. }}$ Let $\mathfrak{\Omega}_{0}=\mathfrak{\Omega}_{\cap} \cap \mathfrak{S}_{1}\left[\mathfrak{Q}_{1}, \mathfrak{X}_{1}\right] \supseteqq \mathfrak{\Omega}_{1}$. Since $X_{1}$ centralizes a subgroup of $\mathfrak{S}_{1}$ of index 2 , we get $\mathfrak{\Omega}_{0}=\mathfrak{\Omega}_{1}$, so $\mathfrak{\Omega}_{1}$ is a

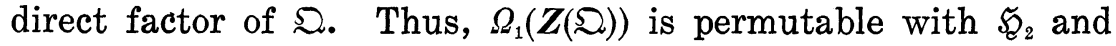

$$
\mathfrak{S}_{2} \Omega_{1}(Z(\mathfrak{\Omega})) \nsubseteq \subseteq \text {. }
$$

Hence, $\mathfrak{\Omega}$ is elementary.

Suppose $|\mathfrak{Q}| \geqq 3^{3}$. Then Hypothesis 13.1 is satisfied and $\mathscr{A}(3) \subseteq$ $\mathscr{C}^{*}(\mathbb{S})$. Let $\mathfrak{M}=M(\mathfrak{Q}) \supseteqq \mathfrak{S}$. Thus, $\mathfrak{S}_{2}$ is a $S_{2}$-subgroup of $\mathbb{S}$ by Lemma 13.8(c). Hence, $\mathfrak{S}_{2}=\mathfrak{I}$, against $\mathfrak{T} \in \mathscr{l}^{*}(\mathbb{S})$. Hence, $|\mathfrak{D}|=3^{a}$, with $a=1$ or 2 .

Suppose by way of contradiction that $a=2$. Since $\mathfrak{Y}$ admits $\cong$, we get $\mathfrak{\Omega}=\mathfrak{\Omega}_{1} \times \mathfrak{\Omega}_{2}$, where $\mathfrak{\Omega}_{2}=C_{\mathfrak{\Omega}}(Y) \subseteq C\left(\mathfrak{X}_{0}\right) \subseteq \mathfrak{S}^{a}$. Hence, $\mathfrak{\Omega}_{2}$ stabilizes $\mathfrak{X} \supset \mathfrak{X}_{0} \supset 1$, so $\mathfrak{\Omega}_{2}$ centralizes $\mathfrak{X}$. Hence, $\mathfrak{X}_{1} \subseteq N_{\mathfrak{\Phi}_{2}}\left(\mathfrak{D}_{2}\right)=\mathfrak{I}_{0}$, say.

Since $\mathfrak{S}_{1} \cong / \mathfrak{S}_{1}$ is a chief factor of $\mathfrak{K}_{\mathcal{C}}, \mathfrak{Q}$ is an irreducible $\mathfrak{T}_{0}$-group. Thus, we can choose $T$ in $\mathfrak{I}_{0}$ such that $\mathfrak{\Omega}_{1}^{T} \neq \mathfrak{\Omega}_{1}$. In particular, 
$\mathfrak{S}_{1} \mathfrak{X}_{1} / \mathscr{S}_{1}$ is not central in $\mathfrak{S}_{2} / \mathfrak{F}_{1}$, so $\mathfrak{X} \neq \mathfrak{B}_{0}$. Since $\mathfrak{X}$ may be chosen as any conjugate of $\mathfrak{B}_{0}$ which lies in $\mathfrak{K}_{2}$ but not in $\mathfrak{S}_{1}$, we get $\mathfrak{B}_{0} \subseteq \mathfrak{S}_{1}$. Since $a=2$, we have $\mathfrak{Q}=\mathfrak{\Omega}_{1} \times \mathfrak{\Omega}_{1}^{T}$. Let $\mathfrak{W}=\left[\mathfrak{S}_{1}, \mathfrak{Q}\right]=\left[\mathfrak{S}_{1}, \mathfrak{\Omega}_{1}\right] \times$ $\left[\mathfrak{S}_{1}, \mathfrak{Q}_{1}^{T}\right]=\mathfrak{Y} \times \mathfrak{Y}^{T}$, of order $2^{4}$. Thus, $T$ does not normalize $\mathfrak{X}$. Let $\mathfrak{W}_{1}=\mathfrak{W} \mathfrak{B} \triangleleft \mathfrak{S}_{\text {. }}$ We argue that $\left|\mathfrak{W}_{1}: \mathfrak{W}\right| \leqq 2$. Suppose false. In any case, $\mathfrak{W}_{1}$ is abelian. Since $\mathfrak{W}_{1}=\mathfrak{W} \times C_{\mathfrak{F}_{1}}(\mathfrak{Q})$, it follows that $\mathfrak{Q}$ centralizes $\sigma^{1}\left(\mathfrak{B}_{1}\right)=\sigma^{1}(\mathfrak{B})$. Hence, $\mathfrak{B}=\mathfrak{B}_{0}$, so that $\mathfrak{W}_{1}=\mathfrak{W} \times \mathfrak{B}$. Let $\mathfrak{W}_{2}=C_{\mathfrak{F}_{1}}(\mathfrak{D})$. Thus, $\mathfrak{W}_{2} \cong \mathfrak{B}$ and $\mathfrak{W}_{2} \cap \mathfrak{B}=1$. We now get $\mathfrak{W}_{1}=\mathfrak{Y} \times \mathfrak{Y}^{T} \times$ $\mathfrak{B}=\mathfrak{Y} \times \mathfrak{Y}^{T} \times \mathfrak{W}_{2}$. Thus, for each $V$ in $\mathfrak{B}^{\sharp}$, we have $V=V_{1} V_{2} W$ with $V_{1} \in \mathfrak{Y}, V_{2} \in \mathfrak{Y}^{T}, W \in \mathfrak{W}_{2}$. If $V_{1}=1$, then $\mathfrak{Q}_{1}$ centralizes $V$, against $\mathfrak{Q} \cap \mathfrak{S}=1$. If $V_{2}=1$, then $\mathfrak{\Omega}_{1}^{T}$ centralizes $\mathfrak{B}$, against $\mathfrak{Q} \cap \mathfrak{S}=1$. Hence, $V_{i} \neq 1, i=1,2$. Hence, $\mathfrak{W}_{1}=\mathfrak{B} \times \mathfrak{Y}^{T} \times \mathfrak{W}_{2}$. This is impossible, since $X_{1}$ centralizes $\mathfrak{B} \times \mathfrak{Y}^{T} \times \mathfrak{W}_{2}$. Hence, $\left|\mathfrak{W}_{1}: \mathfrak{W}\right| \leqq 2$.

Suppose $\left|\mathfrak{W}_{1}: \mathfrak{W}\right|=2$. Then $\mathfrak{W}_{1}=\mathfrak{W} \times 3$, where $3=C_{\mathfrak{R}_{1}}(\mathfrak{Q})$ is of order 2 and is centralized by $\mathfrak{I}_{0} \mathfrak{\Omega}=N_{\mathfrak{5}}(\mathfrak{Q})$. Since $\mathfrak{W}_{1}=\mathfrak{W} \mathfrak{B}$, it follows that $\left[\mathfrak{W}_{1}, \mathfrak{S}_{1}\right]=\left[\mathfrak{B}, \mathfrak{S}_{1}\right]$, since $\mathfrak{W}=\mathfrak{Y} \times \mathfrak{Y}^{T} \subseteq Z\left(\mathfrak{S}_{1}\right)$. Thus, $\left[\mathfrak{W}_{1}, \mathfrak{F}_{1}\right]$ is a normal subgroup of $\mathfrak{S}_{2}$ of order at most 2 , so $\mathfrak{D}$ centralizes $\left[\mathfrak{W}_{1}, \mathfrak{K}_{1}\right]$. Since $\mathfrak{W} \cap C(\mathfrak{D})=1$, we get $\mathfrak{W}_{1} \subseteq Z\left(\mathfrak{S}_{1}\right), 3 \subseteq Z(\mathfrak{S})$.

Let $3=\langle Z\rangle$ and choose $V \in \mathfrak{B}-\mathfrak{W}$. Let $\tilde{\mathfrak{B}}_{0}=\mathfrak{B} \cap \mathfrak{B}=\left\langle V_{0}\right\rangle$. Furthermore, let $\mathfrak{V}=\left\langle Y_{1}, Y_{2}\right\rangle$ with $Y_{1}^{X_{1}}=Y_{2}$. Thus, $\mathfrak{W}=\left\langle Y_{1}\right\rangle \times$ $\left\langle Y_{2}\right\rangle \times\left\langle Y_{1}^{T}\right\rangle \times\left\langle Y_{2}^{T}\right\rangle$. Hence, $\mathfrak{W} \cap C\left(\mathfrak{E}_{2}\right)=Y_{1} Y_{2} Y_{1}^{T} Y_{2}^{T}$. Since $\tilde{\mathfrak{B}}_{0}$ is a normal subgroup of $\mathfrak{K}_{2}$ of order 2 , we get $V_{0}=Y_{1} Y_{2} Y_{1}^{T} Y_{2}^{T}$. Now $V=Z W$ with $W \in \mathfrak{W}$. Let $W=W_{1} W_{2}$, with $W_{1} \in \mathfrak{Y}, W_{2} \in \mathfrak{Y}^{\top}$. If $W_{i}=1$ for $i=1$ or $i=2$, then $\Omega \cap \subseteq \neq 1$. Hence, $W_{i} \neq 1, i=1,2$. Since $[\mathfrak{X}, \mathfrak{B}]=1$, by Lemma 13.41 , we have $\left[X_{1}, W\right]=1$. Hence, $W_{1}=Y_{1} Y_{2}$. Since $\left[\mathfrak{X}^{T}, \mathfrak{B}\right]=1$, we get $W_{2}=Y_{1}^{T} Y_{2}^{T}$. Hence, $Z=V V_{0} \in$ $\mathfrak{B}^{\sharp}$, against $\subseteq \cap \mathfrak{Q}=1$. We conclude that $\mathfrak{B} \subseteq \mathfrak{W}$.

Again, let $\mathfrak{Y}=\left\langle Y_{1}, Y_{2}\right\rangle$ with $Y_{1}^{X_{1}}=Y_{2}$. Since $\mathfrak{B} \subseteq \mathfrak{W} \cap \boldsymbol{C}(\mathfrak{X}) \cap \boldsymbol{C}\left(\mathfrak{X}^{T}\right)$, we get $\mathfrak{B} \subseteq\left\langle Y_{1} Y_{2}, Y_{1}^{T} Y_{2}^{T}\right\rangle=\mathfrak{B} \cap \boldsymbol{C}(\mathfrak{X}) \cap \boldsymbol{C}\left(\mathfrak{X}^{T}\right)$. But then this containment is an equality, since $|\mathfrak{B}|=4$. Hence, $Y_{1}^{T} Y_{2}^{T} \in \mathfrak{B}$. Hence, $C\left(\mathfrak{Y}^{T}\right) \subseteq$ $C\left(Y_{1}^{T} Y_{2}^{T}\right) \subseteq \subseteq$, against $\mathfrak{\Omega}_{1} \subseteq C\left(\mathfrak{Y}^{T}\right), \subseteq \cap \Omega=1$. Hence, $a=1, \mathfrak{\Omega}=\mathfrak{F}$, $\mathfrak{\Omega}=\mathfrak{\Omega}_{1}$.

Suppose $\mathfrak{B} \subseteq \mathfrak{S}_{1}$. Then by Lemma 13.41 , we get $\mathfrak{B} \triangleleft \mathfrak{S}_{1} \cap \boldsymbol{C}(\mathfrak{X})=$ $C_{\mathfrak{S}_{1}}(\mathfrak{Q}) \times \mathfrak{X}_{0}$. Hence, $\mathfrak{B} \cap \boldsymbol{C}_{\mathfrak{S}_{1}}(\mathfrak{\Omega}) \neq 1$, against $\mathfrak{Q} \nsubseteq \mathfrak{S}$. Since $\mathfrak{B} \nsubseteq \mathfrak{S}_{1}$, we may assume that $\mathfrak{B}=\mathfrak{X}$. Choose $U \in N_{\mathfrak{x}}\left(\mathfrak{S}_{2}\right)-\mathfrak{S}_{2}$, with $U^{2} \in \mathfrak{F}_{2}$. Then $U$ normalizes $\mathfrak{B}_{0}$ and also normalizes $C_{\mathfrak{F}_{2}}\left(\mathfrak{B}_{0}\right)=\boldsymbol{C}_{\mathfrak{F}_{1}}(\mathfrak{Q}) \times \mathfrak{B}_{0}$. If $\boldsymbol{C}_{\mathfrak{\mathfrak { 1 }}_{1}}\left(\mathfrak{B}_{0}\right)$ is not elementary, then $\boldsymbol{N}\left(\boldsymbol{D}\left(\boldsymbol{C}_{\mathfrak{\mathfrak { w }}_{1}}(\mathfrak{B})\right)\right) \supseteqq\langle\mathfrak{U}, \mathfrak{S}\rangle$, against the maximality of $\mathfrak{F}_{2}$. Hence, $\boldsymbol{C}_{\mathfrak{w}_{1}}(Q)$ is elementary and so is central in $\mathfrak{S}_{2}$. If

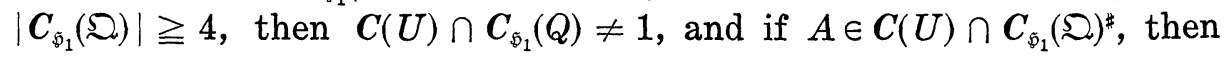
$C(A) \supseteqq\langle U, \mathfrak{S}\rangle$. This is impossible, by maximality of $\mathfrak{S}_{2}$, so $\left|\boldsymbol{C}_{\mathfrak{t}_{1}}(\mathfrak{Q})\right| \leqq 2$. If $\boldsymbol{C}_{\mathfrak{S}_{1}}(\mathfrak{Q})=1$, then $\mathfrak{S}_{2}$ is dihedral of order 8 , against $2 \in \pi_{4}$, and Lemma 13.2(d)(i). Hence, $\left|\boldsymbol{C}_{\mathfrak{W}_{1}}(\mathfrak{D})\right|=2$. In this case, $\mathfrak{S}_{2}$ has precisely 2 elementary subgroups of order 8 , namely, $\mathfrak{S}_{1}$ and $C_{\mathfrak{S}_{2}}\left(\mathfrak{B}_{0}\right)$. Since $U$ nor- 
malizes $\boldsymbol{C}_{\mathfrak{F}_{2}}\left(\mathfrak{B}_{0}\right), U$ also normalizes $\mathfrak{S}_{1}$. This contradiction completes the proof of the theorem.

HYPOTHESIS 13.4 .

(a) $\subseteq \in \mathscr{L} \mathscr{S}(\mathbb{S})$.

(b) $\boldsymbol{O}_{2},($ S) $=1$.

(c) Every normal abelian subgroup of $\subseteq$ is cyclic.

(d) $\quad \mathfrak{S}=\boldsymbol{O}_{2}(\mathfrak{S})$ has a characteristic abelian subgroup 3 of order 8 .

Lemmas 13.54 through 13.57 are proved under Hypothesis 13.4. $\mathfrak{I}$ denotes a $S_{2}$-subgroup of $\mathfrak{S}$. We observe that if (a), (b), (c) of Hypothesis 13.4 hold, then $\Omega_{1}(\boldsymbol{Z}(\mathfrak{I}))=\Omega_{1}\left(\boldsymbol{Z}\left(\boldsymbol{O}_{2}(\mathfrak{S})\right)\right)$, so that $\mathfrak{I}$ is a $S_{2^{-}}$ subgroup of $\mathbb{S}$ and $N(\mathfrak{T}) \subseteq \mathfrak{S}$.

LEMMA 13.54. 3 is a cyclic weakly closed subgroup of $\mathfrak{S}$.

Proof. By Hypothesis 13.4 (c), $\mathfrak{S}$ is of symplectic type, so $3=\langle Z\rangle$ is cyclic. Suppose $G \in \mathbb{B}-\mathfrak{S}$ and $\mathbb{3}^{G}=\mathfrak{X}=\langle X\rangle \subseteq \subseteq$, where $X=Z^{G}$. Let $\mathfrak{Y}=\mathfrak{S} \cap \mathfrak{X}$. If $|\mathfrak{Y}|>2$, then $\Omega_{1}(\mathfrak{Y})=\Omega_{1}(\boldsymbol{D}(\mathfrak{Y})) \subseteq \Omega_{1}(\boldsymbol{D}(\mathfrak{E}))=\left\langle Z^{4}\right\rangle$, so $X^{4}=Z^{4}, G \in \mathfrak{S}$. Hence, $|\mathfrak{Y}| \leqq 2$.

Let $\mathfrak{S}_{\mathcal{C}}=\mathfrak{S}_{0} \mathfrak{S}_{1}$, where $\mathfrak{S}_{0}$ is either cyclic or of maximal class and $\mathfrak{S}_{1}$ is extra special, and $\left[\mathfrak{S}_{0}, \mathfrak{S}_{1}\left[=1\right.\right.$. Let $\mathfrak{S}_{2}$ be the unique normal subgroup of $\mathfrak{S}_{0}$ of order 4 , and let $\Re=\mathfrak{K}_{2} \mathfrak{S}_{1}$ char $\mathfrak{K}_{\text {. }}$. Let $\Re_{0}=C_{\Omega}\left(X^{2}\right)$, so that $\Omega_{0} \subset \Omega$. The containment is proper since $C_{\mathscr{\S}}(\Omega)=C_{\mathfrak{F}}(\Omega)$, this equality holding since every chief factor of $\mathfrak{S}$ between $\mathscr{S}$ and $\mathscr{R}$ is central. Let $\Re_{1} / \Re_{0}$ be a chief factor of $\Re X$ with $\Re_{1} \subseteq \Re$.

Suppose $X^{4}$ centralizes $\Re_{1}$. Then $\left[\Re_{1}, X\right] \subseteq \mathfrak{X} \cap \Re_{1} \subseteq\left\langle X^{4}\right\rangle$, so that $\mathfrak{X}$ stabilizes $\Re_{1} \supset \Re_{1} \cap\left\langle X^{4}\right\rangle \supseteqq 1$. This implies that $X^{2}$ centralizes $\Re_{1}$, against our construction. Hence, $\mathfrak{X}$ acts faithfully on $\Re_{1}$. Choose $K \in \Re_{1}-\Re_{0}$. Let $X^{-1} K X=K K_{0}$, where $K_{0} \in \Re_{0}$. Let $X^{-1} K_{0} X=K_{0} K_{1}$. Thus, $X^{-2} K X^{2}=K K_{0} K_{0} K_{1}=K K_{0}^{2} K_{1}$. Since $K_{0} \in C\left(X^{2}\right) \subseteq N(\mathfrak{X})$, we get $K_{1} \in\left\langle X^{4}\right\rangle$. If $K_{1}=1$, then $X^{4}$ centralizes $K$, so centralizes $\Re_{1}$. This is not the case, so $K_{1}=X^{4}$. Since $D(\Re)=\Omega_{1}(Z(\subseteq))$, we get $K_{0}^{2} \in$ $\Omega_{1}(\boldsymbol{Z}(\mathfrak{S}))$. Hence, $X^{4} K X^{4}=X^{-2} K K_{0}^{2} X^{4} X^{2}=K K_{0}^{2} X^{4} K_{0}^{2} X^{4}=K$, against $\mathfrak{X} \cap C\left(\Re_{1}\right)=1$. This contradiction completes the proof.

Lemma 13.55. $\left\langle Z^{2}\right\rangle=B_{0}$ is weakly in $\subseteq$.

Proof. Suppose $G \in \mathbb{B}-\mathfrak{S}$ and $Y^{G} \in \mathfrak{S}$, where $Y=Z^{2}$. Since Aut (3) is elementary, $Y^{2}$ centralizes 3. Hence, $3 \subseteq C\left(Y^{2}\right)=\mathfrak{S}^{G}$. Hence, $3=3^{G}$, since $3^{G}$ is weakly closed in $\mathfrak{S}^{G}$. This yields $G \in \mathfrak{S}$. The proof is complete.

Let $Y=Z^{2}, I=Y^{2}=Z^{4}$.

Lemma 13.56. $\langle I\rangle$ is weakly closed in $C_{\mathfrak{s}}(Y)$. 
Proof. Suppose $G \in \mathbb{S}-\mathfrak{S}$ and $I^{G}=J \in C_{\oplus}(Y)$. Then $Y \in C(J)=$ $\mathfrak{S}^{G}$. Since $\left\langle Y^{G}\right\rangle$ is weakly closed in $\mathfrak{\Im}^{G}$, we get $\langle Y\rangle=\left\langle Y^{G}\right\rangle, G \in \mathfrak{S}$. The proof is complete.

Lemma 13.57. If $J$ is an involution of $\boldsymbol{C}_{\S}(3)$, then $\boldsymbol{C}(J) \subseteq \subseteq$.

Proof. Suppose false. Let

$$
\mathscr{N}=\left\{\mathfrak{N} \mid \mathfrak{N} \in \mathscr{S} \mathscr{O} \mathscr{L}(\mathfrak{S}), 3 \subseteq \mathfrak{X}, \boldsymbol{O}_{2^{\prime}}(\mathfrak{R})=1, \mathfrak{R} \nsubseteq \mathbb{S}\right\}
$$

Thus, $C(J) \in \mathscr{N}$, by Lemma 13.1. Choose $\mathfrak{R} \in \mathscr{N}^{\prime}$ such that $\mid \mathfrak{N} \cap \Im_{2}$ is maximal. Let $\mathfrak{I}^{*}$ be a $S_{2}$-subgroup of $\mathfrak{R} \cap \mathfrak{S}$. Thus, $3 \subseteq \mathfrak{I}^{*}$. Let $\mathfrak{L}=N\left(\mathfrak{T}^{*}\right)$. By Lemma 13.1, $\boldsymbol{O}_{2^{\prime}}(\mathbb{R})=1$. If $\mathfrak{I}^{*}$ is not a $S_{2^{-} \text {-subgroup }}$ of $\mathfrak{R}$, then $\mathbb{R} \nsubseteq \mathfrak{S}$, so that $\mathbb{R} \in \mathscr{N},|\mathfrak{R} \cap \mathfrak{S}|_{2}>|\mathfrak{R} \cap \mathfrak{R}|_{2}$. This violates the choice of $\mathfrak{R}$, so $\mathfrak{I}^{*}$ is a $S_{2}$-subgroup of $\mathfrak{N}$. Since $\mathfrak{Y}=\left\langle Z^{2}\right\rangle$ is weakly closed in $\mathfrak{S}$, we have $\mathfrak{Y} \nsubseteq \boldsymbol{O}_{2}(\mathfrak{N})$. Hence, $\boldsymbol{O}_{2}(\mathfrak{R}) \cap \mathfrak{Y}=\langle I\rangle$. But $\boldsymbol{O}_{2}(\mathfrak{R}) \subseteq \mathfrak{I}^{*} \leqq \subseteq$, so $\left[\boldsymbol{O}_{2}(\mathfrak{R}), 3\right] \subseteq \boldsymbol{O}_{2}(\mathfrak{N}) \cap 3 \subseteq\langle I\rangle$. This implies that $Z^{2}$ centralizes $\boldsymbol{O}_{2}(\mathfrak{R})$, so $Z^{2}=Y \in \boldsymbol{O}_{2}(\mathfrak{N})$. This contradiction completes the proof.

Theorem 13.5. Hypothesis 13.4 is not satisfied.

Proof. Suppose false. By a basic result of Glauberman [16], there is $G$ in $\left(\mathbb{S}-\mathfrak{S}\right.$ such that $I^{G}=J \in \mathfrak{T}$. Let $\mathbb{F}=C_{\mathfrak{x}}(J)$. Thus, $\mathfrak{F}$ normalizes 3 and $3^{G}$. Let $\mathfrak{F}_{0}=C_{\mathbb{E}}(Z)$, $\mathfrak{F}_{1}=C_{\mathbb{E}}\left(Y^{G}\right)$. Suppose $\mathfrak{\Im}_{0} \cap \mathfrak{F}_{1} \neq 1$. Choose $E \in E_{0} \cap E_{1}^{\#}$. By Lemma 13.56, $C(E) \subseteq \subseteq$. Hence, $Y^{G} \in \mathfrak{S}$. Since $\langle Y\rangle$ is weakly closed in $\mathfrak{S}$, we get $\left\langle Y^{G}\right\rangle=\langle Y\rangle, I=I^{G}$. Hence, $\mathfrak{F}_{0} \cap \mathfrak{F}_{1}=1$. Since $\boldsymbol{D}(\mathfrak{\xi}) \leqq \mathfrak{F}_{0} \cap \mathfrak{F}_{1}$, we get that $\boldsymbol{D}(\mathfrak{G})=1$. Since

$$
|\operatorname{Aut}(3)|=4,|\operatorname{Aut}(\langle Y\rangle)|=2 \text {, }
$$

we get $|\mathfrak{F}| \leqq 8$. Since $2 \in \pi_{4}$, we conclude that $|\mathfrak{F}|=8$.

Let $\mathfrak{S}^{*}=C_{\mathfrak{F}}(Z)$. Thus, $\left|\mathfrak{F}^{*} \cap C(J)\right|=2$, since $\mathfrak{S}^{*} \cap C(J)$ is faithfully represented on $\left\langle Y^{G}\right\rangle$. Hence, $\mathfrak{S}^{*}\langle J\rangle$ is of maximal class. Thus, $\mathfrak{S}_{\mathfrak{C}}^{*}$ is either of maximal class or is cyclic. Since $Z \in Z\left(\mathfrak{S}^{*}\right)$, we get that $\mathfrak{S C}^{*}$ is cyclic. This is not the case, since the width of $\mathfrak{F}$ is at least 1 . The proof is complete.

HYPOTHESIS 13.5 .

(a) $\subseteq \in \mathscr{L} \mathscr{S}(\mathbb{S})$.

(b) $\boldsymbol{O}_{2^{\prime}}(\mathfrak{S})=1$.

(c) Every normal abelian subgroup of $\subseteq$ is cyclic.

(d) $\mathfrak{S}=\boldsymbol{O}_{2}(\mathfrak{S})$ contains a characteristic subgroup 3 of order 4 . Lemmas 13.58 through 13.60 are proved under Hypothesis 13.5. Set $3=\langle Z\rangle, I=Z^{2}, 3_{0}=\langle I\rangle$. Let $\mathfrak{I}$ be a $S_{2}$-subgroup of $\mathfrak{S}$. Let $w$ be 
the width of $\mathfrak{S}$. Thus, $\mathfrak{F}$ is the central product of 3 and an extra special group of width $w$, by Theorem 13.5. Since $2 \in \pi_{4}$, we have $w \geqq 2$, so that $m(\mathfrak{S})=2 w+1 \geqq 5$.

LEMma 13.58. If $\mathfrak{S}_{1}$ is a subgroup of $\mathfrak{S}_{\mathrm{g}}$ of index 2 which contains 3 , then for each $G \in \mathbb{B}-\mathfrak{S}, \mathfrak{S}_{1}^{G} \nsubseteq \mathbb{S}$. $\mathfrak{X}=\langle X\rangle$.

Proof. Suppose false. Let $\Re=\mathfrak{S E}^{G}, \Re_{1}=\mathfrak{S}_{1}^{G}, \mathfrak{X}=3^{G}, J=I^{G}=X^{2}$,

Case 1. $J \notin \mathfrak{S}$.

Since $\left|\Re: \Re_{1}\right|=2, \Re_{1}$ contains an extra special subgroup $\Re_{0}$ of width $w$ - 1. Thus, $\Re_{0} \cap \mathfrak{S}=1$, since $J$ is the only minimal normal subgroup of $\Re_{0}$. Let $\mathbb{L}=\boldsymbol{O}_{2,2^{\prime}}(\mathfrak{S}), \mathfrak{S}^{*}=[\mathfrak{S}, \mathbb{R}]$. Thus, $\mathfrak{S C}^{*}$ is extra special of width $w^{*} \leqq w$. By Lemma 5.13, we get $w \leqq 2$. Hence, $w=2$, since $2 \in \pi_{4}$. However, $\Omega_{0} \mathfrak{X}$ is the central product of $\Omega_{0}$ and $\mathfrak{X}$ and $\Re_{0} \mathfrak{X}$ is faithfully represented on $\mathfrak{L} / \mathfrak{S}_{\mathcal{C}}$. Let $\mathfrak{L}=\mathfrak{S}_{\mathfrak{C}} \mathfrak{R}_{0}$, where $\mathfrak{L}_{0}$ is a $S_{2}$-subgroup of $\mathbb{R}$. Thus, $\mathbb{\Omega}_{0}$ is faithfully represented on $\mathfrak{S}^{*}$, an extra special group of width 2 . Thus, $\left|\Omega_{0}\right|=3,5$ or 9 . This is impossible, as $\Omega_{0} \mathfrak{X}$ is isomorphic to a subgroup of $\operatorname{Aut}\left(\Omega_{0}\right)$.

\section{Case 2. $J \in \mathfrak{S}$.}

Let $\mathfrak{S}_{0}=C_{\mathfrak{f}}(J)$, so that $\left|\mathfrak{S}_{\mathfrak{C}}: \mathfrak{S}_{0}\right|=2$. Let $\mathbb{F}=\mathfrak{F} \cap \Re_{1}$. Then

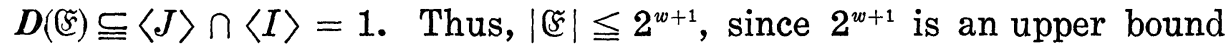
for the order of every elementary subgroup of $\mathfrak{S}$. Let $|\mathbb{F}|=2^{e}$. Since $\Re /\langle J\rangle$ is elementary, so is $\Omega_{1} / \mathfrak{S}$. Thus, $\Re_{1} / \mathfrak{S}$ is elementary of order $2^{2 w+1-e}$. Now $\Re_{1} / \mathfrak{F} \cong \Re_{1} \mathscr{E} / \mathscr{S}=\bar{\Re}_{1}$, and $\bar{\Re}_{1}$ is represented faithfully on $\overline{\mathfrak{L}}=\boldsymbol{O}_{2,2^{\prime}}(\mathfrak{S}) / \mathfrak{L}$. Set $f=2 w+1-e$. Thus, $\overline{\mathfrak{L}}$ contains a subgroup $\overline{\mathfrak{B}}=\overline{\mathfrak{B}}_{1} \times \cdots \times \overline{\mathfrak{B}}_{f}$, such that $\left|\overline{\mathfrak{B}}_{i}\right|=p_{i}$ is a prime, $\overline{\mathfrak{B}}_{i}$ admits $\overline{\mathfrak{R}}_{1}$, $1 \leqq i \leqq f$, and $C_{\bar{R}_{1}}(\bar{B})=1$, and where we have chosen $\mathfrak{B}$ as an abelian subgroup of $\boldsymbol{O}_{2,2^{\prime}}(\mathfrak{S})$ of odd order, such that $\overline{\mathfrak{B}}=\mathfrak{B} \mathfrak{E} / \mathfrak{S}, \mathfrak{B}_{i} \cong \mathfrak{B}, \overline{\mathfrak{B}}_{i}=$ $\mathfrak{B}_{i} \mathfrak{S} / \mathfrak{S}$.

Let $V=\mathfrak{S} / \mathscr{S C}^{\prime}$, so that $V$ is elementary of order $2^{2 w+1}$. Let $V=$ $V_{0} \times V_{1}$, where $V_{0}=C_{V}(\mathfrak{B}), V_{1}=[V, \mathfrak{B}]$. Thus, $\mathbb{Z} / \mathfrak{S}^{\prime} \subseteq V_{0}$, so $\left|V_{1}\right|=2^{a}$ with $a \leqq 2 w$.

Since $e \leqq w+1$, we get $f \geqq w$. Let $\mathfrak{2}_{0}=\left\langle\mathfrak{B}, \mathfrak{S}_{2}, \mathfrak{R}_{1}\right\rangle$, and let $\mathfrak{A}=\boldsymbol{A}_{\mathfrak{x}_{0}}\left(V_{1}\right)$. Thus, $\mathfrak{Q}=\mathfrak{R}_{1} \times \cdots \times \mathfrak{A}_{f}$, where $\mathfrak{A}_{i}$ is dihedral of order $2 p_{i}$, and we choose notation so that $\mathfrak{A}_{i}$ is the image of $\left\langle\mathfrak{B}_{i}, K_{i}, \mathfrak{K}\right\rangle$ in $\mathfrak{A}$, where $K_{i} \in \Re_{1}$. Let $V_{1}=W_{1} \supset W_{2} \supset \cdots \supset W_{s+1}=1$ be a composition series for $V_{1}$ as $\mathfrak{Q}$-group. Let $W^{i}=W_{i} / W_{i+1}, i=1,2, \cdots, s$, and let $\mathfrak{H}^{i}=C_{\mathfrak{r}}\left(W^{i}\right)$. Since $\mathfrak{N}_{1}^{\prime}, \cdots, \mathfrak{R}_{f}^{\prime}$ exhaust all the minimal normal subgroups of $\mathfrak{X}$, we get that $\mathfrak{X}^{i}=\mathfrak{A}_{J(i)}$ for some subset $J(i)$ of $\{1, \cdots, f\}$, 
and where we define $\mathfrak{A}_{J}=\left\langle\mathfrak{A}_{j} \mid j \in J\right\rangle$ for all $J \subseteq\{1, \cdots, f\}$. Thus, each $W^{i}$ is a free $F_{2} \Re^{i}$-module, where $\Re^{i}$ is a $S_{2}$-subgroup of $\mathfrak{A} / \mathfrak{A}^{i}$. Hence, $\left|W^{i}\right| \geqq 2^{\left|\Omega^{i}\right|}$ for all $i$, so that $\left|W_{1}\right| \geqq 2^{k}$, where $k=\sum_{i=1}^{s}\left|\Re^{i}\right|$. Since $\left|\Re^{i}\right| \geqq 2$ for each $i$, it follows that if $\left|\Re^{i}\right|=2^{i}$, then $\left|\Re^{i}\right| \geqq 2 b_{i}$. Hence, $2 w \geqq k \geqq 2 \sum b_{i}$. Since $\sum b_{i} \geqq f \geqq w$, we conclude that $k=$ $2 w, 2 b_{i}=\left|\Re^{i}\right|$ for all $i$. Hence, $b_{i}=1$ or 2 for each $i$. This implies that $\mathfrak{B}$ is a 3 ,5-group, and that for each $i$, a $S_{3,5}$-subgroup of $\mathfrak{A} / \mathfrak{N}^{i}$ is of order 3 or 15 .

We can do a little better, however. Namely, $\mathfrak{O}$ is not represented faithfully on any proper submodule of $V_{1}$, as the inequalities show. Let $\mathfrak{M}(2)$ be the $S_{3,5}$-subgroup of $\mathfrak{M} \cap C\left(W_{2}\right)$, so that $\mathfrak{U}(2) \neq 1$. Let $\mathfrak{B}(2)$ be the preimage of $\mathfrak{U}(2)$ in $\mathfrak{B}$. Then set $V_{1}=\mathfrak{S}^{*} / \mathfrak{S}^{\prime}$. Hence, $\mathfrak{S}^{*}=\boldsymbol{C}_{\mathfrak{F}^{*}}(\mathfrak{B}(2))\left[\mathfrak{S}^{*}, \mathfrak{B}(2)\right], W_{2}=\boldsymbol{C}_{\mathfrak{F}^{*}}(\mathfrak{B}(2)) / \mathfrak{S}^{\prime}$ and $\left[\mathfrak{F}^{*}, \mathfrak{B}(2)\right] / \mathfrak{S}^{\prime}$ is a complement $W^{1}$ to $W_{2}$ in $V_{1}=W_{1}$. Hence, $W_{1}=W^{1} \times W_{2}$ as R-module. Repeating this argument suitably often implies that $V_{1}=W^{1} \times W^{2} \times$ $\cdots \times W^{s}, \mathfrak{S}_{2}^{*}=\mathfrak{S}_{1}^{*} \cdots \mathfrak{S}_{s}^{*}$, where each $\mathfrak{S}_{i}^{*}$ is extra special, admits $\mathfrak{S C B R} \Re_{1}$, and $W^{i}=\mathfrak{S}_{i}^{*} / \mathfrak{S}^{\prime}$ is an irreducible $\mathfrak{A}$-module. Since $\left|W^{i}\right| \leqq 2^{4}$, it follows that Aut $\left(\mathfrak{S}_{i}^{*}\right)$ does not have a subgroup of order 15 . Hence, $\mathfrak{B}$ is a 3 -group, and $\mathfrak{K}_{i}^{*}$ is the central product of $b_{i}$ quaternion groups, $1 \leqq i \leqq s$. By the inequalities, we get $3=C_{\mathfrak{w}}(\mathfrak{B})$, so that

$$
\mathfrak{S}_{\mathfrak{E}}=3 \mathfrak{S}_{1}^{*} \cdots \mathfrak{S}_{s}^{*} \text {. }
$$

Case 2a. $w \geqq 3$.

Since $f=w$ and $\mathfrak{B}$ is elementary of order $3^{w}$, we get $e(3) \geqq 3$. Hence, $\mathscr{A}(3) \subseteq \mathscr{C}^{*}(\mathbb{S})$ and so $\mathscr{S}=\mathbb{M}(\mathfrak{B})$. Also, since $e=w+1$, $\mathbb{F}=\Re_{1} \cap \mathfrak{F}$ is an elementary subgroup of $\mathfrak{S}$ such that $3 \mathfrak{F} \in \mathscr{S} \mathscr{C} \mathscr{N}(\mathfrak{S})$. Suppose $E \in \mathfrak{F}-\langle I\rangle$ and $C_{\mathfrak{B}}(E)$ is non cyclic. Let $\mathfrak{F}=\langle E, I\rangle$. Thus, $C_{\mathfrak{B}}(F)$ is non cyclic for each $F \in \mathfrak{F}^{*}$. Since $\mathscr{A}(3) \leqq \mathbb{Z}^{*}(\mathbb{S})$, we get $C(F) \subseteq \subseteq$ for all $F \in \mathfrak{F}^{\prime}$. By Case 1 applied to $\mathfrak{S}^{G}$, we get $I \in \mathfrak{S C}^{G}$. Hence, $\mathfrak{F} \subseteq \mathfrak{S C}^{G}$, and so $\mathfrak{S C}^{G}=\left\langle\mathfrak{S C}^{G} \cap C(F) \mid F \in \mathfrak{F}^{\sharp}\right\rangle \subseteq \subseteq$. Hence, $\mathfrak{S C}_{\mathfrak{C}}{ }^{G}$ is a 2-subgroup of $\subseteq$ whose center is contained in $3 \cap 3^{G}=1$. This is impossible, so no such $E$ exists.

Again, since $f=w$, it follows that $\mathfrak{F}$ is the central product of 3 with quaternion groups $\mathfrak{\Omega}_{1}, \cdots, \mathfrak{\Omega}_{w}$ such that each $\mathfrak{\Omega}_{i}$ admits $\mathfrak{B}$. Let $\mathfrak{S}_{i}=\mathfrak{B} \cap C\left(\mathfrak{\Omega}_{i}\right)$, so that $\left|\mathfrak{B}: \mathfrak{S}_{i}\right|=3,1 \leqq i \leqq w$. For each subset $J$ of $\{1, \cdots, w\}$, let $\mathfrak{\Omega}_{J}=\left\langle 3, \mathfrak{\Omega}_{i} \mid i \in J\right\rangle$. As $J$ ranges over all the subsets of $\{1, \cdots, w\}$, the groups $\mathfrak{D}_{J}$ range over all the subgroups of $\mathfrak{S}$ which contain 3 and admit $\mathfrak{B}$.

Suppose $i, j \in\{1, \cdots, w\}, i \neq j$. Let $J^{\prime}=\{i, j\}, J=\{1, \cdots, w\}-J^{\prime}$. Then $\mathfrak{F} \cap \mathfrak{\Omega}_{J}=\langle I\rangle$, as we have shown, since

$$
\bigcap_{\substack{k=1 \\ k \neq i, j}}^{w} \mathfrak{S}_{k}
$$


is non cyclic and centralizes $\mathfrak{Q}_{J}$. Thus, for each $E \in \mathfrak{F}, E=$ $E(J) E\left(J^{\prime}\right)$, where $E(J) \in \mathfrak{\Omega}_{J}, E\left(J^{\prime}\right) \in \mathfrak{\Omega}_{J^{\prime}}$, and $E\left(J^{\prime}\right) \in \mathbb{Z}$ if and only if $E \in\langle I\rangle$. Thus, the map $\varphi: E \mapsto E\left(J^{\prime}\right) 3$ is a homomorphism of $\&$ into $\mathfrak{\Omega}_{J^{\prime}} / \mathbb{3}$ with $\operatorname{ker} \varphi=\langle I\rangle$. Since $\left|\mathfrak{\Omega}_{J^{\prime}}: 3\right|=2^{4}$, we get $|\mathfrak{F}| \leqq 2^{5}$. Since $|\mathfrak{F}|=2^{w+1}$, we have $w=3$ or 4 .

We next show that $\mathfrak{S}^{a} \cap \mathfrak{B}=1$. Namely,

$$
\left[\mathfrak{S}^{G} \cap \mathfrak{B}, \mathfrak{R}_{1}\right] \subseteq \mathfrak{S} \mathfrak{C} \cap \mathfrak{S}^{G} \subseteq \mathfrak{K} \text {. }
$$

Since $\Re_{1}$ has no non trivial fixed points on $\mathscr{S} \mathfrak{E} / \mathscr{S}$, by construction, we get $\mathfrak{S}^{G} \cap \mathfrak{B}=1$. We may rewrite this equation in the equivalent form $C_{\mathfrak{B}}(J)=1$. Hence, we have $J=Z^{a} Q_{1} Q_{2} \cdots Q_{w}$, where $Q_{i} \in \mathfrak{\Omega}_{i}-$ $\langle I\rangle, 1 \leqq i \leqq w$.

Now $\overline{\mathfrak{B}}=\mathfrak{B} \mathscr{S} / \mathfrak{S}=\overline{\mathfrak{C}} \times \overline{\mathfrak{D}}$, where $\bar{X}=\mathfrak{F} X$ inverts $\overline{\mathfrak{C}}$ and centralizes D. Suppose $|\overline{\mathfrak{C}}| \geqq 9$. Let $\mathbb{C}$ be a subgroup of $\mathfrak{B}$ incident with $\overline{\mathfrak{C}}$. Thus, $X$ normalizes $[\mathscr{F}, \mathbb{E}]=\tilde{\mathfrak{F}}$, and $3 \tilde{B}=Q_{J}$ for some $J$. Since

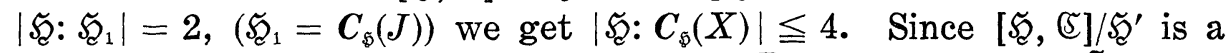
free $F_{2}\langle X\rangle \mid\langle J\rangle$-module, it follows that $|\widetilde{\mathfrak{C}}|=9$, and that $\tilde{\mathfrak{F}}$ is the central product of two quaternion groups. Since $w \geqq 3, J \notin[\mathfrak{K}$, (5)]. Hence, $\left[X, C_{\tilde{\mathfrak{F}}}(J)\right] \subseteq \tilde{\mathcal{F}} \cap\langle J\rangle=1$, so that $X$ centralizes a subgroup of $\widetilde{\mathfrak{F}}$ of index 2. This is not the case, so $|\overline{\mathfrak{E}}| \leqq 3$.

Since $w=f \geqq 3$, we get $|\bar{D}| \geqq 9$. Let $\mathfrak{D}$ be a subgroup of $\mathfrak{B}$ incident with $\overline{\mathfrak{D}}$. Thus, $\langle X, \mathfrak{S}\rangle \triangleleft\langle X, \mathfrak{S}, \mathfrak{D}\rangle$. Hence, $\boldsymbol{D}(\langle X, \mathfrak{S}\rangle)=$ $\left\langle X^{2}, \mathfrak{S}_{\mathcal{E}},\left[\mathfrak{S E}_{\mathcal{E}}, X\right]\right\rangle$ is normalized by $\mathfrak{D}$. Since $\left[\mathfrak{S}_{1}, X\right] \subseteq\left\langle X^{2}\right\rangle=\langle J\rangle$, it follows that $|\boldsymbol{D}(\langle X, \mathfrak{S}\rangle)| \leqq 8$. Hence, $\mathfrak{D} \cap \boldsymbol{C}(J) \neq 1$, against $\mathfrak{B} \cap \mathfrak{S}^{G}=1$. This contradiction shows that this case does not occur.

\section{Case $2 b . \quad w \leqq 2$.}

Here we get $w=2$, since $2 \in \pi_{4}$. Hence, $|\xi|=2^{3}$, where, as above, $\mathfrak{F}=\mathfrak{S} \cap \Re_{1}$.

Let $\tilde{\mathbb{L}}=N_{\mathscr{\odot}}(\mathfrak{B})$. Since $S_{2}^{\prime}$-subgroups of Aut $\left(\mathfrak{S}^{*}\right)$ are of order 9 , it follows that $\mathfrak{B}$ is a $S_{2^{\prime}}$-subgroup of $\widetilde{\mathfrak{L}}$. This implies that $\mathfrak{S}=\mathfrak{S} \widetilde{\mathbb{R}}$, since Aut $(\mathfrak{S})$ has no elements of order 15, and by hypothesis, $\subseteq$ is solvable. Thus, $\mathfrak{S}^{*} \triangleleft \mathfrak{S}$. Since $\mathfrak{S}^{*}$ has exactly 2 quaternion subgroups, it follows that $\mathfrak{I} / \mathfrak{S}$ is isomorphic to a subgroup of a dihedral group of order 8 . Since $\Re_{1} \Re / \mathfrak{S} \cong \Re_{1} / \mathbb{F}$ is a four-group, $\mathfrak{I} / \mathfrak{S}$ is dihedral of order 4 or 8 .

Choose generators $B_{1}, B_{2}$ for $\mathfrak{B}$ such that $C_{\mathfrak{F}^{*}}\left(B_{i}\right)=\mathfrak{D}_{i}$ is a quaternion group, $i=1,2$. Suppose $J \in 3^{2}$. Then $B_{i}$ centralizes $J$, so $B_{i} \in \mathfrak{S}^{G}$. Hence, $\left[\Re_{1}, B_{i}\right] \subseteq \mathscr{S}^{G}$, against the fact that $\Re_{1}$ has no non

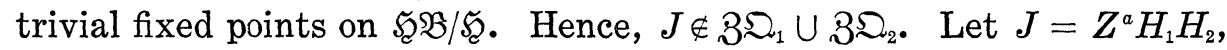
where $H_{i} \in \mathfrak{\Omega}_{i}$. Thus, $H_{1}$ and $H_{2}$ are both of order 4 , so $H_{1} H_{2}$ is an involution. Hence, $Z^{a} \in\langle I\rangle$, and replacing $H_{1}$ by $Z^{a} H_{1}$, we get that $J=H_{1} H_{2}$ with $H_{i} \in Q_{i}-\langle I\rangle$. 
Since $\Re_{1} \mathfrak{S}_{\mathfrak{C}} / \mathfrak{S}$ is a four-group, $\Re_{1}$ contains an element $K_{1}$ such that $K_{1}$ inverts $\mathfrak{S} \mathfrak{S B} / \mathfrak{S}$. The coset $\mathbb{5} K_{1}$ is uniquely determined by this condition, since $K_{1}$ lies in a uniquely determined coset of $\mathfrak{K}_{\mathfrak{Z}}$ in $\mathfrak{S}_{\mathfrak{2}} \Re_{1}$. Since $K_{1}$ inverts $\mathfrak{S}_{\mathcal{C}} B_{i}, i=1,2$, it follows that $K_{1}$ normalizes $\mathfrak{D}_{i}, i=1,2$. We can therefore choose $L_{i} \in \mathfrak{Q}_{i}$ such that $\left\langle L_{i}, L_{i}^{K_{1}}\right\rangle=\mathfrak{\Omega}_{i}$. Since $\boldsymbol{D}\left(\Re_{1}\right)=\langle J\rangle$, we get $K_{1}^{2} \in\langle J\rangle$.

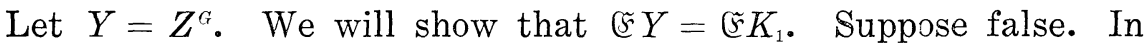
this case, $Y$ does not invert $\mathfrak{S} \mathfrak{B} / \mathfrak{S}$. First, suppose $Y$ normalizes $\mathfrak{D}_{1}$. Since $\Omega_{1}$ and $\Omega_{2}$ are the only quaternion subgroups of $\mathfrak{K}^{*}, Y$ normalizes $\mathfrak{\Omega}_{2}$. If $Y$ induces an inner automorphism of $\mathfrak{\Omega}_{i}$, then $J=Y^{2}$ centralizes $\mathfrak{D}_{i}$. This is not the case, so $Y$ induces outer automorphisms of both $\mathfrak{\Omega}_{1}$ and $\mathfrak{\Omega}_{2}$. Hence, $Y K_{1}$ induces an inner automorphism of $\mathfrak{\Omega}_{1}$ and of $\mathfrak{\Omega}_{2}$, so $Y K_{1}$ induces an inner automorphism of $\mathfrak{S}^{*}$, so $Y K_{1} \in \mathfrak{S}$, which gives $Y K_{1} \in \mathfrak{S}$. We may therefore assume that $Y$ does not normalize $\mathfrak{\Omega}_{1}$. Let $\mathfrak{\Omega}_{1}=\left\langle H_{11}, H_{12}\right\rangle$, and set $H_{2 i}=H_{1 i}^{Y}, i=$ 1,2 , so that $\Omega_{2}=\left\langle H_{21}, H_{22}\right\rangle$. Thus, $H_{2 i}^{Y}=H_{1 i}^{Y^{2}}=H_{1 i}^{J}$. We assume notation is chosen so that $H_{1}=H_{11}$, recalling that $J=H_{1} H_{2}$ with $H_{i} \in \mathfrak{\Omega}_{i}-\langle I\rangle$. Hence, $H_{2}=H_{21}^{ \pm 1}$, and $H_{21}^{Y}=H_{1}^{J}=H_{1}$, while $H_{22}^{Y}=$ $H_{12}^{J}=H_{12}^{-1}$. Since $H_{12} H_{22} \in C(J)$, we get $\left[H_{12} H_{22}, Y\right] \in\langle J\rangle$, as $\langle Y\rangle \triangleleft \Im^{(i)}$. But $H_{22}^{-1} H_{12}^{-1} Y^{-1} H_{12} H_{22} Y=I \notin\langle J\rangle$. We conclude that $\left[5 Y=\sqrt{5} K_{1}\right.$, so that $Y$ inverts $\mathfrak{S} \mathfrak{B} / \mathfrak{S}$.

Since $Y$ inverts $\mathfrak{S} \mathfrak{B} / \mathfrak{F}$, it follows that $Y$ normalizes $\mathfrak{\beth}_{1}$ and $\mathfrak{\Omega}_{2}$ and $Y$ induces an outer automorphism of each. Hence, we can choose generators $H_{i 1}, H_{i 2}$ of $\mathfrak{S}_{i}$ such that $H_{i 1}^{Y}=H_{i 2}, i=1,2$. Hence,

$$
C_{\mathfrak{\wp}^{*}}(Y) \subseteq\left\langle H_{11} H_{12}, H_{21} H_{22}\right\rangle,
$$

an abelian group of type $(2,4)$. Since $\left[\mathscr{S}_{1}, Y\right] \subseteq\langle J\rangle$, it follows that $\boldsymbol{C}_{5^{*}}(Y)=\left\langle H_{11} H_{12}, H_{21} H_{22}\right\rangle$. Since $Y$ centralizes (5, we conclude that ⿷匚 $¥ \mathfrak{S}_{2}^{*}$. Since $H_{11} H_{21} \in \mathfrak{S}_{1}$, we get

$$
\left[H_{11} H_{21}, Y\right]=J=H_{21}^{-1} H_{11}^{-1} Y^{-1} H_{11} H_{21} Y=H_{21}^{-1} H_{11}^{-1} H_{12} H_{22}=H_{11} H_{12} H_{21} H_{22} \text {, }
$$

an important equality.

Since $C_{\mathfrak{5}}(Y)=\left\langle Z, H_{11} H_{12}, H_{21} H_{22}\right\rangle$, and since $\langle I, J\rangle \subset$ (5, we get that $E=Z H_{11} H_{12} \in \mathfrak{\Im}$. Choose $K \in \Re_{1}-\llbracket\langle Y\rangle$. Then $K$ does not centralize $E$, since $Z\left(\Re_{1}\right)=\langle Y, I\rangle$. Hence, $[E, K]=J$, and so $\Omega_{1}^{K}=\Omega_{2}$. Also, however, we conclude that $\Re_{1} \mathfrak{S}$ normalizes $\mathfrak{F}$ and permutes $\mathfrak{5}-\langle I, J\rangle$ transitively.

Let $\widetilde{\Im}=\left\langle C_{\mathfrak{5}}(E), B_{1}, Y\right\rangle \subseteq C_{\mathfrak{\Im}}(E)$. Now $C_{\mathfrak{g}}(E)=\langle E\rangle \times \tilde{\mathscr{\mathcal { E }}}$, where $\tilde{\mathfrak{F}}$ is the central product of 3 and $\mathfrak{\Omega}_{2}$. We can choose $\tilde{H} \in \tilde{\mathfrak{F}}$ such that $\widetilde{H} Y$ inverts $B_{1}$, since $Y$ inverts $\left\langle\tilde{\mathfrak{B}}, B_{1}\right\rangle / \tilde{\mathfrak{F}}$. Hence, $(\widetilde{H} Y)^{2} \in \tilde{\mathfrak{F}} \cap$ $C\left(B_{1}\right)=\langle E\rangle \times\langle Z\rangle$. Let $H=E^{a} Z^{b} H_{21}^{c} H_{22}^{d}$, so that

$$
(\widetilde{H} Y)^{2}=\widetilde{H} J Y^{-1} \tilde{H} Y=\widetilde{H} J \cdot E^{a} Z^{b} H_{22}^{c} H_{21}^{-d}=J Z^{2 b} H_{21}^{c} H_{22}^{d} H_{22}^{c} H_{21}^{-d} \cdot
$$


Hence, $c+d$ is odd, which implies that $(\widetilde{H} Y)^{4}=I$. Hence, $I$ is a fourth-power in $C_{\Im}(E)$. Let $\tilde{\mathfrak{I}}$ be a $S_{2}$-subgroup of $C_{\Im}(E)$. We argue that $\langle I\rangle=\sigma^{2}(\tilde{\mathfrak{I}})$. Namely, $C_{\mathscr{E}}(E)$ does not contain a $S_{3}$-subgroup of $\mathfrak{S}$, since $3=C_{\mathfrak{F}}(\mathfrak{B})$. Hence, $\left\langle B_{1}\right\rangle$ is a $S_{3}$-subgroup of $C_{\mathfrak{E}}(E)$. This

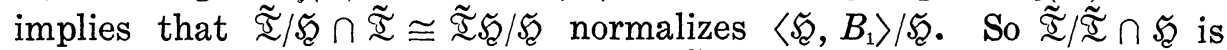
elementary, from which we get $\sigma^{1}(\widetilde{\mathfrak{T}}) \subseteq \mathfrak{H}, \mathscr{O}^{2}(\widetilde{T}) \subseteq \sigma^{1}(\mathfrak{S})=\langle I\rangle$, as required. Since $\langle I\rangle=\sigma^{2}(\tilde{\mathfrak{I}})$, we get that $\tilde{\mathfrak{I}}$ is a $S_{2}$-subgroup of $\boldsymbol{C}(E)=\sqrt{ }$, say. Hence, $\langle I\rangle^{\complement}$ is elementary, since $I \in \boldsymbol{Z}\left(\boldsymbol{O}_{2}(\mathfrak{夭})\right)$. Since $\boldsymbol{A}_{\mathscr{F}}(\mathfrak{F})$ permutes transitively $\mathfrak{F}-\langle I, J\rangle$, it follows that for each $F \in$ $\left(F-\langle I, J\rangle,\langle I\rangle^{c(F)}\right.$ is elementary.

We now exploit the symmetry between $\subseteq$ and $\mathfrak{S}^{G}$. Namely,

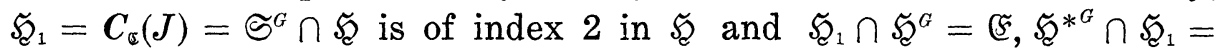
$\langle I, J\rangle$. By all the above applied with the pair $\left(\mathfrak{F}_{1}, \mathbb{S}^{G}\right)$ in the role of the pair $\left(\Re_{1}, \mathfrak{S}\right)$, we get that $\langle J\rangle^{C\left(F^{2}\right)}$ is elementary for all $F \in(\mathfrak{F}-$ $\langle I, J\rangle$. This is not the case, since $\mathfrak{\Omega}_{2} \subseteq\langle J\rangle^{C(E)}$. The proof is complete.

LEMMA 13.59. 〈I $\langle$ is weakly closed in $\mathfrak{S}$.

Proof. Suppose $G \in \mathbb{S}-\mathfrak{S}$ and $J=I^{G} \in \mathfrak{S}$. Let $\mathfrak{S}_{1}=C_{\mathfrak{F}}(J)$ so that $\left|\mathfrak{S}_{\mathfrak{c}}: \mathfrak{S}_{1}\right|=2, \mathfrak{F}_{1} \subseteq \mathfrak{S}^{G}$. By Lemma 13.58, we get $G^{-1} \in \mathfrak{S}$. This is not the case, since $G \in \mathbb{B}-\mathfrak{S}$.

Lemma 13.60. $\mathfrak{T} \in \mathscr{C}^{*}(\mathfrak{S})$.

Proof. Suppose false and $\mathfrak{N}$ is minimal subject to

$$
\mathfrak{I} \subseteq \mathfrak{R} \in \mathscr{S} \mathscr{O} \mathscr{L}(\mathfrak{S}), \quad \mathfrak{N} \varsubsetneqq \text {. }
$$

Thus, as usual, $\mathfrak{R}=\mathfrak{I} \mathfrak{N}$, where $\mathfrak{Q}$ is a $q$-group for some odd prime $q$. Let $\mathfrak{R}_{0}=\boldsymbol{O}_{2}(\mathfrak{N})$. We argue that $3 \subseteq \mathfrak{N}_{0}$. Suppose false. Then $3 \cap \mathfrak{N}_{0}=\langle I\rangle=Z(\mathfrak{T})$ and $\mathfrak{N}_{0} Z$ is central in $\mathfrak{T} / \mathfrak{N}_{0}$. By minimality of $\mathfrak{N}, \mathfrak{N}_{0} \mathfrak{\Omega} / \mathfrak{R}_{0} \boldsymbol{D}(\mathfrak{\Omega})$ is a chief factor of $\mathfrak{R}$, and $\boldsymbol{D}(\mathfrak{\Omega})=\mathfrak{\Omega} \cap \mathfrak{S}$. Thus, $Z$ inverts $\mathfrak{R}_{0} \mathfrak{D} / \mathfrak{R}_{0} \boldsymbol{D}(\mathfrak{\Omega})$ and $Z$ centralizes $\mathfrak{R}_{0} \boldsymbol{D}(\mathfrak{\Omega}) / \mathfrak{M}_{0}$. Since

$$
\left|\mathfrak{N}_{0}: \mathfrak{N}_{0} \cap C(Z)\right| \leqq 2,
$$

we get that $|\mathfrak{Q}|=3, \mathfrak{I}=\mathfrak{R}_{0} \mathfrak{Z}, \mathfrak{N}_{0}=\mathfrak{N}_{\mathfrak{N}_{0}}(\mathfrak{Q}) \times\left[\mathfrak{N}_{0}, \mathfrak{Q}\right]$, and $\left[\mathfrak{N}_{0}, \mathfrak{Q}\right]$ is a fourgroup. Hence, $Z(\mathfrak{I})$ is non cyclic. As this statement is false, we conclude that $3 \subseteq \mathfrak{R}_{0}$. Let $\mathfrak{S}_{0}=\mathfrak{S}_{\mathcal{Z}} \cap \mathfrak{R}_{0} \supseteqq \mathbb{Z}$, so that $\mathfrak{S}_{2} / \mathfrak{S}_{0} \cong \mathfrak{S}_{2} \mathfrak{R}_{0} / \mathfrak{R}_{0}$ is elementary. If $\mathfrak{K}=\mathfrak{S}_{0}$, then $\boldsymbol{Z}\left(\mathfrak{N}_{0}\right) \subseteq \boldsymbol{Z}\left(\mathfrak{S}_{\mathfrak{c}}\right)=\mathbb{3}$, so that $\mathfrak{R} \subseteq \mathfrak{S}$, against our choice of $\mathfrak{R}$. Hence, $\mathfrak{S}_{0} \subset \mathfrak{S}$, and we can choose a subgroup $\mathfrak{\Omega}_{0}$ of $\mathfrak{Q}$ such that (a) $\left|\mathfrak{N}_{0}\right|=q$, (b) $\mathfrak{S}$ normalizes $\mathfrak{R}_{0} \mathfrak{Q}_{0}$, (c) $\mathfrak{K}$ does not centralize $\mathfrak{R}_{0} \mathfrak{Q}_{0} / \mathfrak{R}_{0}$. Let $\mathfrak{L}=\mathfrak{R}_{0} \mathfrak{\Omega}_{0} \mathfrak{S}_{\mathfrak{E}}, \mathfrak{R}_{1}=\boldsymbol{O}_{2}(\mathbb{R}), \mathfrak{S}_{1}=\mathfrak{R}_{1} \cap \mathfrak{S}$, so that $\left|\mathfrak{S}_{2}: \mathfrak{S}_{1}\right|=2$. Clearly $\mathfrak{\Omega}_{0} \nsubseteq \mathfrak{S}$, since $\left[\mathfrak{\Omega}_{0}, \mathfrak{S}_{2}\right]$ is not a 2-group. Let $\mathfrak{\Omega}_{0}=\langle Q\rangle$. Then $\mathfrak{S}_{1}^{Q} \subseteq \mathfrak{R}_{1} \subseteq \mathfrak{T}$, so by Lemma 13.58, $Q \in \mathfrak{S}$. This contradiction completes the proof. 


\section{THEOREM 13.6. Hypothesis 13.5 is not satisfied.}

Proof. Suppose false. By Lemma 13.60, $\mathfrak{T} \in \mathscr{L}^{*}(\mathbb{S})$. Let $\mathfrak{S}$ be the subgroup given in Lemma 13.2. Let $\mathfrak{S}_{2}=\mathfrak{S} \cap \mathfrak{T}$. Thus $Z(\mathfrak{T}) \subseteq$ $\boldsymbol{Z}\left(\boldsymbol{O}_{2}(\mathfrak{S})\right)$, so that $3 \subseteq \mathfrak{F}_{2}$. Let $\mathfrak{B}=V\left(\operatorname{ccl}_{\mathscr{B}}(\mathfrak{Z}) ; \mathfrak{F}_{2}\right)$. By maximality of $\mathfrak{S}_{2}$, we get $\mathfrak{B} \not \mathfrak{S}_{c}$. Hence, there is $G$ in $\mathbb{B}$ such that $\mathfrak{X}=3^{G} \subseteq \mathfrak{S}_{2}$, $\mathfrak{X} \nsubseteq \boldsymbol{O}_{2}(\mathfrak{S})$.

We assume without loss of generality that if $\mathfrak{S}_{2} \subseteq \mathfrak{S}_{3} \subset \mathfrak{S}_{\mathcal{S}}$, then $\mathfrak{S}_{3} \subseteq \mathfrak{S}$. Thus, $\mathfrak{S E}_{2}=\mathfrak{S}_{2} \mathfrak{Q}, \mathfrak{Q}$ is a 3 -group, $\mathfrak{S} \cap \mathfrak{Q}=\boldsymbol{D}(\mathfrak{Q})$, and if $\mathfrak{S}_{0}=$ $\boldsymbol{O}_{2}(\mathfrak{E})$, then $\mathfrak{S}_{0} \mathfrak{O} / \mathfrak{S}_{0} \boldsymbol{D}(\mathfrak{O})$ is a chief factor of $\mathfrak{S}_{\text {. }}$

Let $\mathfrak{X}=\langle X\rangle$, and let $\mathfrak{\Omega}_{0}$ be a subgroup of $\mathfrak{\Omega}$ minimal subject to (a) $\mathfrak{\Omega}_{0} \nsubseteq D(\mathfrak{D})$, (b) $\mathfrak{X}$ normalizes $\mathfrak{S}_{0} \mathfrak{\Omega}_{0}$, (c) $\mathfrak{X}$ does not centralize $\mathfrak{S}_{0} \mathfrak{S}_{0} / \mathfrak{\Omega}_{0}$. Let $\mathfrak{L}=\mathfrak{S}_{0} \mathfrak{\Omega}_{0} \mathfrak{X}, \mathfrak{Q}_{1}=\boldsymbol{O}_{2}(\mathfrak{R})$ and let $\mathfrak{W}_{1}=\Omega_{1}\left(\boldsymbol{Z}\left(\mathfrak{Q}_{1}\right)\right) \supseteqq \Omega_{1}(\boldsymbol{Z}(\mathfrak{T}))$. Thus, $\mathfrak{\Omega}_{0}$ does not centralize $\mathfrak{W}_{1}$. Let $\mathfrak{W}$ be a minimal normal subgroup of $\mathbb{Z}$ which is not centralized by $\mathfrak{\Omega}_{0}$. Since $\mathfrak{L}_{1} \mathfrak{\Omega}_{0} / \mathfrak{Q}_{1} \boldsymbol{D}\left(\mathfrak{\Omega}_{0}\right)$ is a chief factor of $\mathbb{R}$, it follows that $\boldsymbol{C}_{\mathfrak{Q}_{0}}(\mathfrak{W}) \subseteq \boldsymbol{D}\left(\Omega_{0}\right)$. We argue that $X^{2} \in \mathfrak{R}_{1}$. Suppose false. Then the minimal polynomial of $X$ on $\mathfrak{W}$ is a multiple of $(c-1)^{3}$. Hence, $X$ does not centralize $\mathfrak{B} \cap C\left(X^{2}\right)$. But

$$
\left[X, \mathfrak{W} \cap C\left(X^{2}\right)\right] \subseteq\left\langle X^{2}\right\rangle,
$$

so $X^{2} \in \mathfrak{W} \subseteq \mathfrak{Q}_{1}$. Hence, $X^{2} \in \mathfrak{Q}_{1}$, so $\mathfrak{\Omega}_{0}$ is cyclic. Since $X^{2} \in C(\mathfrak{W})$, we get $[\mathfrak{W}, X]=\left\langle X^{2}\right\rangle$, so that $|\mathfrak{W}|=4, D\left(\Omega_{0}\right) \subseteq C\left(X^{2}\right)$. Hence, $X$ centralizes $\boldsymbol{D}\left(\mathfrak{\Omega}_{0}\right)$, and $X$ inverts $\mathfrak{R}_{1} \boldsymbol{D}\left(\mathfrak{\Omega}_{0}\right) / \mathfrak{R}_{1}$, so $\left|\mathfrak{\Omega}_{0}\right|=3$. Since $X^{2} \in Z\left(\mathbb{\Omega}_{1}\right)$, we get $\left[\mathfrak{Q}_{1}, X\right]=\left\langle X^{2}\right\rangle$. Hence, $\mathfrak{\Omega}_{1}=C_{\mathfrak{\Omega}_{1}}\left(\mathfrak{\Omega}_{0}\right) \times \mathfrak{W}$.

Since $\mathfrak{I}$ is not of maximal class, $C_{\mathbb{R}_{1}}\left(\mathfrak{\Omega}_{0}\right) \neq 1$. Suppose $3 \subseteq \mathfrak{S}_{0}$. Then since $\mathfrak{\Omega}_{0}$ centralizes $\widetilde{J}^{1}\left(\mathfrak{Q}_{1}\right)$, we get $\mathfrak{\Omega}_{0} \subseteq C(I)=\mathfrak{S}$. Hence, $3 \nsubseteq \mathfrak{K}_{0}$, and we may assume that $3=\mathfrak{X}$. Hence, $\mathfrak{\Omega}_{0}=\mathfrak{\Omega}, \mathfrak{E}=\mathfrak{S}_{2} \mathfrak{\Omega}, \mathfrak{S}_{2}=$ $\mathfrak{S}_{0}\langle Z\rangle$. Let $\boldsymbol{O}_{2}(\mathfrak{S}) \cap \mathfrak{S}_{0}=\mathfrak{A}$, and let $L$ be an involution in $Z(\mathfrak{E})$. Thus, $\mathfrak{H}_{2}$ is a $S_{2}$-subgroup of $\boldsymbol{C}(L)$, so $\boldsymbol{C}(L) \cap \boldsymbol{O}_{2}(\mathcal{S}) \cong \mathfrak{S}_{2}$. Hence, $\boldsymbol{O}_{2}(\mathcal{S}) \cap \mathfrak{H}_{0} \supset$ $\langle I\rangle$. Suppose $\boldsymbol{O}_{2}(\mathfrak{S}) \cap \boldsymbol{C}_{\mathfrak{S}_{0}}(\mathfrak{Q}) \neq 1$. Then $\boldsymbol{O}_{2}(\mathfrak{S}) \cap \boldsymbol{C}_{\mathfrak{S}_{0}}(\mathfrak{\Omega}) \cap \boldsymbol{Z}(\mathfrak{S})$ contains an involution $L_{0}$. Hence, $\boldsymbol{C}\left(L_{0}\right) \cap \boldsymbol{O}_{2}(\mathfrak{S}) \subseteq \mathfrak{F}_{2}$. Hence, $\boldsymbol{O}_{2}(\mathfrak{S}) \cap \boldsymbol{C}\left(L_{0}\right)$ is abelian, since

$$
\boldsymbol{O}_{2}(\mathfrak{S}) \cap \boldsymbol{C}\left(L_{0}\right)=\left(\boldsymbol{O}_{2}(\mathfrak{S}) \cap \boldsymbol{C}\left(L_{0}\right) \cap \mathscr{S}_{0}\right)\langle Z\rangle \text { and } \boldsymbol{O}_{2}(\mathfrak{S}) \cap \boldsymbol{C}\left(L_{0}\right) \cap \mathscr{S}_{0}
$$

is elementary. This is not the case, since the width of $\boldsymbol{O}_{2}(\mathfrak{S})$ is at least 2. Hence, $\boldsymbol{O}_{2}(\mathfrak{S}) \cap \boldsymbol{C}_{\mathfrak{w}_{0}}(\mathfrak{\Omega})=1$. Let $U=I^{Q} \in \mathbb{Z}\left(\mathfrak{H}_{0}\right)$. Thus, $\mathrm{O}_{2}(\subseteq) \cap C(U) \subseteq N(\mathfrak{W})$, so that $\mathrm{O}_{2}(\Im) \cap C(U) \subseteq \mathfrak{S}_{2}$ and $Z \notin O_{2}(\Im) \cap C(U)$, since $[U, Z]=Z^{2}=I$. Since $\boldsymbol{O}_{2}(\mathfrak{S}) \cap \boldsymbol{C}_{\mathfrak{\Im}_{0}}(\mathfrak{\Omega})=1$, and since $\boldsymbol{O}_{2}(\mathfrak{S}) \cap$ $\boldsymbol{C}(U) \leqq \mathfrak{S}_{0}$, it follows that $\boldsymbol{O}_{2}(\mathfrak{S}) \cap \boldsymbol{C}(U)$ is a four-group. But $m\left(\boldsymbol{O}_{2}(\widetilde{S})\right) \geqq$ 5 , so $\left|O_{2}(\Im) \cap C(U)\right| \geqq 8$. This contradiction completes the proof.

HYPOTHESIS 13.6.

(a) $\mathfrak{S} \in \mathscr{M} \mathscr{S}(\mathbb{S})$.

(b) $\boldsymbol{O}_{2^{\prime}}(\mathfrak{S})=1$, and every normal abelian subgroup of $\mathfrak{S}$ is cyclic. 
(c) $\boldsymbol{O}_{2}(\mathrm{~S})$ is extra special.

Lemmas 13.61 through 13.69 are proved under Hypothesis 13.6. Let $\mathfrak{S}=\boldsymbol{O}_{2}(\mathfrak{S})$, let $w$ be the width of $\mathfrak{S}$, and let $\mathfrak{I}$ be a $S_{2}$-subgroup of $\mathfrak{S}$. Let $\mathfrak{S}^{\prime}=\langle I\rangle=Z(\mathfrak{S})$. These equations show that $N(\mathfrak{I}) \subseteq \mathfrak{S}$, so that $\mathfrak{I}$ is a $S_{2}$-subgroup of $(\mathfrak{S}$. Let $\mathfrak{\Im}$ be the set of non central involutions of $\mathfrak{F}_{\mathcal{C}}$. Since $2 \in \pi_{4}$, we have $w \geqq 2$. $\langle I, X\rangle$.

LEMmA 13.61. If $X \in \mathfrak{\Im}$ and $\mathfrak{S}_{1}=C_{\mathfrak{5}}(X)$, then $C\left(\mathfrak{S}_{1}\right)=Z\left(\mathfrak{V}_{1}\right)=$

Proof. Since $I \in \mathfrak{S}_{1}$, we have $C\left(\mathscr{S}_{1}\right)=C_{\mathfrak{S}}\left(\mathscr{S}_{1}\right)$, so it suffices to show that $\boldsymbol{C}_{\mathfrak{S}}\left(\mathfrak{F}_{1}\right) \subseteq \mathfrak{S}_{\text {. }}$. Now $\mathfrak{S}_{1}=\langle X\rangle \times \mathfrak{S}_{2}$, where $\mathfrak{S}_{2}$ is extra special, and $C_{\mathfrak{S}}\left(\mathscr{S}_{2}\right)=\mathscr{K}_{3}$, where $\mathfrak{S}_{3}$ is dihedral of order 8 .

Choose $C \in C\left(\mathfrak{S}_{1}\right)$. Then $C$ stabilizes $\mathfrak{K}_{2} \supset \mathscr{S}_{1} \supset 1$, so $C$ is a 2-element. Also, $C$ normalizes $C_{\mathfrak{5}}\left(\mathfrak{S}_{2}\right)=\mathfrak{S}_{3}$ and $C$ centralizes the four-subgroup $\langle I, X\rangle$ of $\mathfrak{S}_{3}$. Hence, $C$ induces an inner automorphism of $\mathfrak{S}_{3}$, so $C$ induces an inner automorphism of $\mathfrak{F}$. Since $\boldsymbol{C}_{\mathfrak{S}}(\mathfrak{F})=\langle I\rangle$, we have $C \in \mathfrak{S}$.

LemMA 13.62. If $\mathfrak{S}_{1}$ is any subgroup of index 2 in $\mathfrak{F}_{\text {, then }} \mathfrak{S}_{1}$ contains every involution of $C\left(\mathfrak{S}_{1}\right)$, and $C\left(\mathfrak{S}_{1}\right)$ is a 2-group.

Proof. Since $\mathfrak{S}_{2}$ is extra special, $\left|\boldsymbol{Z}\left(\mathfrak{S}_{1}\right)\right|=4$. If $\boldsymbol{Z}\left(\mathfrak{S}_{1}\right)$ is a fourgroup, we are done by Lemma 13.61 , so suppose $Z\left(\mathfrak{S}_{1}\right)$ is cyclic. Let $X$ be an involution of $\boldsymbol{C}\left(\mathfrak{S}_{1}\right)$. Then $X \in \mathfrak{S}$, and $\mathfrak{S}_{1}=\boldsymbol{Z}\left(\mathfrak{S}_{1}\right) \mathfrak{S}_{2}$, where $\mathfrak{S}_{2}$ is extra special, and $X$ normalizes $C_{\mathfrak{5}}\left(\mathfrak{S}_{2}\right)=\mathfrak{S}_{3}$. If $\mathfrak{S}_{3}$ is a quaternion group, then $X$ induces an inner automorphism of $\mathfrak{S}_{3}$, so that $X$ induces an inner automorphism of $\mathfrak{S}$ and we are done. We may assume that $\mathfrak{S}_{3}$ is dihedral and that $X$ induces an outer automorphism of $\mathfrak{S}_{3}$. Hence, $X \notin \mathfrak{S}$, so by Lemma 5.36, $X$ inverts an element $Q$ of $\mathfrak{S}$ of odd prime order. Let $\mathfrak{Q}=\langle Q\rangle$. Since $X$ centralizes $\mathfrak{S}_{1}$, and $\left|\mathfrak{S}: \mathfrak{S}_{1}\right|=2$, we get $|\mathfrak{Q}|=3$, and $[\mathfrak{F}, \mathfrak{Q}]=\tilde{\mathfrak{F}}$ is a quaternion group. Thus, $X$ induces an outer automorphism of $\tilde{\mathfrak{F}}$, against $\left|\mathfrak{F}_{\mathcal{C}}: \mathfrak{S}_{1}\right|=2$. The proof is complete.

The next lemma is somewhat elaborate, and is the nub of the matter.

Lemma 13.63. Suppose $X \in \mathfrak{I}, \mathscr{F}_{1}=C_{\mathfrak{g}}(X)$ and $G \in \mathbb{S}-\mathfrak{S}$. Then $\mathfrak{S}_{1}^{G} \nsubseteq \mathfrak{S}$.

Proof. Suppose false. Let $\Re_{1}=\mathfrak{F}_{1}^{G} \subseteq \mathfrak{S}$. We assume without loss of generality that $\Re_{1} \subseteq \mathfrak{T}$. By Lemma 13.61, $C\left(\mathfrak{S}_{1}\right)=\langle X, I\rangle$. Set $J=I^{G}$, so that $C\left(\Re_{1}\right)=\left\langle X^{G}, J\right\rangle$. Since $I \in Z(\subseteq)$, we get $I \in\left\langle X^{G}, J\right\rangle$. Hence, $\Re_{1}=\langle I\rangle \times \Re_{2}$, where $\Re_{2}$ is extra special of width $w-1$. 
Suppose $\mathfrak{S} \cap \Re_{1}=\langle I\rangle$. Then $\mathscr{S} \cap \Re_{2}=1$. By Lemma 5.12, $w=2$. Since $\Re_{2}$ is represented faithfully on $\boldsymbol{O}_{2,2^{\prime}}(\mathfrak{S}) / \mathfrak{K}$, it follows that $\boldsymbol{A}_{\mathscr{S}}\left(\mathfrak{S}_{\mathcal{E}}\right)$ contains a $S_{2}$-subgroup of Aut $(\mathfrak{S})$. It follows that $\mathfrak{I}$ does not normalize any elementary subgroup of $\mathfrak{S}$ of order 8 . On the other hand, $2 \in \pi_{4}$, so $\mathfrak{I}$ contains a normal elementary $\mathfrak{F}$ of order 8 . Let $\mathfrak{F}_{0}=$ $\mathfrak{F} \cap \mathfrak{F}$. If $\mathfrak{F}_{0}=\langle I\rangle$, then $\mathfrak{F}$ stabilizes $\mathfrak{S} \supset\langle I\rangle \supset 1$. This is not the case since $\mathfrak{S} / \mathfrak{F}$ is represented faithfully on $\mathfrak{S} / \mathfrak{S g}^{\prime}$. Hence, $\mathfrak{F}_{0}$ is a fourgroup. Choose $F \in \mathfrak{F}-\mathfrak{F}_{0}$. If $F$ normalizes each of the two quaternion subgroups of $\mathfrak{S}_{2}$, then since $\left[F, \mathfrak{S}_{2}\right] \subseteq \mathfrak{F}_{0}, F$ induces inner automorphisms of each, so $F$ induces an inner automorphism of $\mathfrak{S}$. This is not the case, since $F \notin \mathfrak{F}$. Hence, $\mathfrak{F}$ interchanges the 2 quaternion subgroups of $\mathfrak{F}$. This implies that $\left|\left[\mathfrak{H}_{\mathcal{C}}, F\right]\right|=8$, against $\left[\mathfrak{F}_{\mathcal{C}}, F\right] \subseteq \mathfrak{F}_{0}$. This contradiction completes a proof that $\Re_{1} \cap \mathfrak{S} \supset\langle I\rangle$.

Since $\langle J\rangle$ is the only minimal normal subgroup of $\mathscr{K}_{2}$, and since $1 \subset \Re_{2} \cap \mathfrak{K} \triangleleft \Re_{2}$, we get $J \in \mathfrak{F}$.

Set $\mathbb{F}=\Re_{1} \cap \mathfrak{E} \supseteqq\langle I, J\rangle$. Since $\Re_{1} /\langle J\rangle$ is elementary, $\Re_{1} / \mathfrak{F}$ is also elementary. Let $|\mathfrak{F}|=2^{e},\left|\Re_{1}: \mathfrak{F}\right|=2^{f}$. Since $D(\mathfrak{F}) \subseteq\langle I\rangle \cap\langle J\rangle=1$, ऽ and $\Re_{1} / \mathscr{\Im}$ are both elementary. Since $\left|\mathfrak{S}^{G}: \Re_{1}\right|=2$, we have $e+f=2 w$.

Let $\mathfrak{I}=\mathfrak{S}(\mathfrak{S})$ be the set of all non central involutions $I_{1}$ of $\mathfrak{S}$

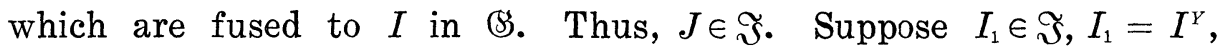
$Y \in$ (S). Then $C\left(I_{1}\right)=\mathfrak{S}^{Y}$ and $\left|\mathfrak{S}: C_{\mathfrak{5}}\left(I_{1}\right)\right|=2$. By the preceding argument we get $I \in \mathfrak{S}^{Y}$, so that $I \in \mathfrak{\Im}^{Y}$. This implies that $C_{\mathfrak{F}^{Y}}(I) \subseteq \mathfrak{S}$, and $\left|\mathscr{S}^{Y}: C_{\mathscr{S}^{Y}}(I)\right|=2$. Let $\mathscr{P}$ be the set of all ordered pairs $(A, B)$, $A \sim B \sim I$, such that $A \in \Im(C(B))$. By what we have just shown, $\mathscr{P}$ is symmetric. This symmetry is quite exploitable.

We now study $\Re_{1}$ in greater detail.

Let $\mathfrak{B}=\mathfrak{B}_{1} \times \cdots \times \mathfrak{B}_{f}$ be a subgroup of $F(\mathfrak{S} \bmod \mathfrak{S})$ such that (a) $\left|\mathfrak{B}_{i}\right|=p_{i}$, an odd prime, (b) $\mathfrak{S C B}_{i}$ admits $\Re_{1}$, (c) $C_{\mathscr{S}_{1}}(\mathfrak{S} \mathfrak{B} / \mathfrak{F})=$ (5. The existence of $\mathfrak{B}$ is guaranteed by Lemma 5.34. For each subset $\mathfrak{A}$ of $\mathfrak{S}$, let $\overline{\mathfrak{A}}=\mathfrak{A} \mathfrak{S} / \mathfrak{S}$. Let $\mathfrak{\Omega}_{i}$ be the subgroup of $\mathfrak{R}_{1}$ containing $\mathbb{F}$ such that $\overline{\mathfrak{R}}_{i}=C_{\mathfrak{R}_{1}}\left(\mathfrak{B}_{i}\right), 1 \leqq i \leqq f$, so that $\left|\Re_{1}: \mathfrak{R}_{i}\right|=2$. Let $\mathfrak{R}^{i}=$ $\bigcap_{j \neq i} \mathfrak{B}_{i}$, and let $\mathfrak{D}_{i}=\left\langle\mathfrak{Q}^{i}, \mathfrak{B}_{i}, \mathfrak{S}\right\rangle$. Then $\overline{\mathfrak{D}}_{i}$ is dihedral of order $2 p_{i}$, and if we set $\mathbb{R}=\mathfrak{S E}_{\mathfrak{B}} \mathbb{R}_{1}$, then $\overline{\mathfrak{L}}=\mathfrak{D}_{1} \times \cdots \times \mathfrak{D}_{f}$. Let $V=\mathfrak{S} / \mathfrak{S}^{\prime}$ so that $V$ is a faithful $F_{2}$ \&-module. This notation will be preserved throughout the remainder of this lemma.

Case 1. $e=w(=f)$.

By Lemma 5.14, $p_{i}=3$ for all $i$.

Since $\mathfrak{B}$ is elementary of order $3^{w}$ and $\mathfrak{B}$ is represented faithfully on $\mathfrak{F}$, it follows that $\mathfrak{F}$ is the central product of quaternion groups $\mathfrak{\Omega}_{1}, \cdots, \mathfrak{\Omega}_{w}$, each of which admits $\mathfrak{B}$. 
Case 1a. $w \geqq 3$.

Here we have $e(3) \geqq 3$, so $\mathscr{A}(3) \subseteq \mathscr{C}^{*}(\mathbb{S})$. By Theorem 13.1, $S_{3}$ subgroups of $\subseteq \cap \mathfrak{S}^{G}$ are of order 1 or 3. Suppose $\mathfrak{S}^{G} \cap \mathfrak{B} \neq 1$. Choose $B \in \mathfrak{S}^{G} \cap \mathfrak{B}^{\sharp}$. Since $\mathfrak{B} \in \mathscr{A}(3)$, we get $C(B) \subseteq \subseteq$. Hence, $C_{\Im^{a}}(B) \subseteq \subseteq$, so $S_{3}$-subgroups of $\subseteq \cap \mathfrak{S}^{G}$ are non cyclic. We conclude that $\mathfrak{S}^{G} \cap \mathfrak{B}=1$. Let $J=Q_{1} \cdots Q_{w}, Q_{i} \in \mathfrak{\Omega}_{i}$. Since $\bigcap_{j \neq i} C_{\mathfrak{g}}\left(\mathfrak{\Omega}_{j}\right)$ is of order 3 for each $i$, we get that $Q_{i} \in \mathfrak{\Omega}_{i}-\langle I\rangle$, all $i$. Since $J$ is an involution, $w$ is even, so $w \geqq 4$.

Suppose $E \in\left(\xi-\langle I\rangle\right.$, and $E \in \Omega_{1} \ldots \Omega_{w-2}$. Since $C_{23}\left(\Omega_{1} \ldots \Omega_{w-2}\right) \in$ $\mathscr{A}(3)$, we get $C(E) \leqq \subseteq, C(E I) \leqq \subseteq$. In particular,

$$
\mathfrak{S}^{G}=\left\langle C_{\mathfrak{\xi}^{G}}(E), C_{\mathfrak{g}^{G}}(E I), C_{\mathfrak{\xi}^{G}}(I)\right\rangle \subseteq \subseteq .
$$

Hence, $\mathfrak{S} \cdot \mathfrak{S C}^{G}$ is a 2-subgroup of $\mathfrak{S}$ whose center is contained in $\langle I\rangle \cap$ $\langle J\rangle=1$. This is impossible, so $\mathfrak{F} \cap \mathfrak{D}_{1} \ldots \mathfrak{Q}_{w-2}=\langle I\rangle$. For each $E$ in $\mathfrak{F}$, let $E=E_{1} E_{2}, E_{1} \in \mathfrak{\Omega}_{1} \ldots \mathfrak{\Omega}_{w-2}, E_{2} \in \mathfrak{\Omega}_{w-1} \mathfrak{N}_{w}$. The map $\varphi: \mathfrak{s} \rightarrow$ $\mathfrak{\Omega}_{w-1} \mathfrak{\Omega}_{w} /\langle I\rangle$, given by $\varphi(E)=\langle I\rangle E_{2}$ is a homorphism with $\operatorname{ker} \varphi=$ $\langle I\rangle$. Hence, $|\mathfrak{s}:\langle I\rangle| \leqq 2^{4}$, so that $w-1 \leqq 4, w \leqq 5$. As $w$ is even, we have $w=4$.

The preceding argument yields that $\mathfrak{F} \cap \mathfrak{D}_{i} \mathfrak{\Omega}_{j}=\langle I\rangle$ for all $i, j$. Since $\mathfrak{B}$ acts faithfully on $\mathfrak{F}$ and does not act faithfully on any proper subgroup of $\mathfrak{S}_{2},\left\{\mathfrak{N}_{1}, \mathfrak{\Omega}_{2}, \mathfrak{\Omega}_{3}, \mathfrak{\Omega}_{4}\right\}$ is the set of all quaternion subgroups of $\mathfrak{K}$ which admit $\mathfrak{B}$. Hence, $N_{\mathfrak{R}}(\mathfrak{B})$ permutes $\left\{\mathfrak{N}_{1}, \mathfrak{\Omega}_{2}, \mathfrak{\Omega}_{3}, \mathfrak{N}_{4}\right\}$. Since $\mathfrak{Q}=\mathfrak{F} \cdot N_{\mathfrak{R}}(\mathfrak{B}), \Re_{1}$ permutes $\left\{\mathfrak{\Omega}_{1}, \mathfrak{\Omega}_{2}, \mathfrak{\Omega}_{3}, \mathfrak{\Omega}_{4}\right\}$. We can choose $K \in \mathfrak{R}_{1}$ such that $\bar{K}$ inverts $\overline{\mathfrak{B}}$. Hence, $K$ normalizes each $\mathfrak{\Omega}_{i}$, and $K$ induces outer automorphisms of each $\mathfrak{\Omega}_{i}$. We can choose generators $Q_{i 1}, Q_{i 2}$ of $\mathfrak{Q}_{i}$ such that $Q_{i 1}^{K}=Q_{i 2}$. Let $\tilde{\mathfrak{Q}}_{i}=\left\langle Q_{i 1} Q_{i 2}\right\rangle$. Since $K$ centralizes $J=$ $Q_{1} \cdots Q_{w}$, we have $Q_{1} \cdots Q_{w}=J=Q_{1}^{K} \cdots Q_{w}^{K}$, so that $Q_{i} \in \tilde{\mathfrak{D}}_{i}$, all $i$. Hence, $J$ centralizes $Q=Q_{11} Q_{21}$, since $J$ does not centralize either $Q_{11}$ or $Q_{21}$. Hence, $Q \in \mathbb{S}^{G}$, so that $[Q, K] \subseteq \mathfrak{S E}^{G} \cap \mathfrak{S}=\mathfrak{F}_{\text {. }}$ But

$$
[Q, K]=Q_{21}^{-1} Q_{11}^{-1} K Q_{11} Q_{21} K=Q_{21}^{-1} Q_{11}^{-1} Q_{12} Q_{22} \in(\widetilde{s}-\langle I\rangle) \cap \mathfrak{D}_{1} \mathfrak{\Omega}_{2} .
$$

This contradiction shows that this case does not arise.

$$
\text { Case 1b. } w \leqq 2 .
$$

Here we get $w=2$, as $2 \in \pi_{4}$. Since $f=2$, a $S_{2^{\prime}}$-subgroup $\mathfrak{B}$ of $S$ is elementary of order 9 , and $\mathfrak{S} \mathfrak{B}$ permutes $\mathfrak{\Im}$ transitively. First, suppose that for some $A \in \mathbb{B}-\mathfrak{S}, \mathfrak{S}^{A} \cap \mathfrak{F}=\mathfrak{F}$ is elementary of order 8. Since $\mathfrak{K}$ has just 6 elementary subgroups of order 8 , $\mathfrak{B}$ contains a subgroup $\mathfrak{B}_{0}$ of order 3 which normalizes $\mathfrak{F}$. Hence, $A_{\circledast}(\mathfrak{F})$ contains the stability groups of $\mathfrak{\Im} \supset\langle I\rangle \supset 1$ and $\mathfrak{F} \supset\left\langle I^{A}\right\rangle \supset 1$ and contains $\boldsymbol{A}_{\mathfrak{B}}(\mathfrak{F}) \neq 1$. Hence, $\boldsymbol{A}_{\mathfrak{\Theta}}(\mathfrak{F})$ is non solvable. We may therefore assume 
that whenever $I^{A}=I_{1} \in \mathfrak{I}(\mathfrak{S})$, then $\mathfrak{F}^{A} \cap \mathfrak{S}$ is a four-group.

Suppose $\mathfrak{S} \mathfrak{B} \Re_{1} \subset \mathfrak{S}$. In this case, $\mathfrak{I} / \mathfrak{F}$ is dihedral of order 8 , and it follows as in a previous argument that $\mathscr{S} \mathscr{C} \mathscr{N}_{3}(\mathfrak{I})=\varnothing$. Hence, $\subseteq=\mathfrak{S}_{\mathfrak{B}} \Re_{1}$ is of order $2^{7} \cdot 3^{2}$. Since $\Re_{1}=\langle I\rangle \times \Re_{2}$, where $\Re_{2}$ is dihedral of order 8 , we can choose non commuting involutions $D_{1}, D_{2}$ of $\Omega_{2}$.

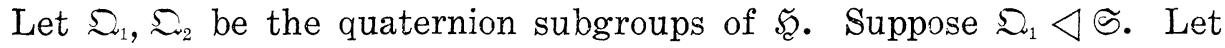
$B_{i}$ be an element of $\subseteq$ of order 3 which is inverted by $D_{i}, i=1,2$. If $D_{1}$ and $D_{2}$ both induce outer automorphisms of $\mathfrak{\Omega}_{1}$ and $\mathfrak{\Omega}_{2}$, then $D_{1} D_{2}$ induces an inner automorphism of $\mathfrak{F}$, against $D_{1} D_{2} \notin \mathfrak{F}$. Hence, we may assume that $D_{1}$ induces an inner automorphism of $\mathfrak{\Omega}_{1}$ and an outer automorphism of $\Omega_{2}$. Choose generators $Q_{i 1}, Q_{22}$ of $\Omega_{i}$ such that $Q_{11}^{D_{2}}=Q_{12}, Q_{21}^{D_{1}}=Q_{22}$. Now $J=Q_{1} Q_{2}$, with $Q_{i} \in \mathfrak{\Omega}_{i}-\langle I\rangle$. Since $D_{1}$ inverts $Q_{21} Q_{22}, D_{1}$ inverts $Q_{2}$, so $D_{1}$ inverts $Q_{1}$. Since $D_{1}$ induces an inner automorphism of $\bigcap_{1}$, we get $Q_{1 i}^{D_{1}}=Q_{1 i}^{d_{2}}, d_{i}= \pm 1, i=1$, 2. Since $Q_{1}$ is a generator for $\left\langle Q_{11} Q_{12}\right\rangle$, we get $Q_{11}^{d_{1}} Q_{12}^{d_{2}}=Q_{12}^{-1} Q_{11}^{-1}=Q_{11} Q_{12}^{-1}$. Hence, $d_{1} d_{2}=-1$. Thus, we can choose $i \in\{1,2\}$ such that $Q_{1 i}^{D_{1}}=Q_{1 i}^{-1}$. Let $\widetilde{F}=\left\langle D_{1}, I, J\right\rangle \subseteq \mathfrak{S}^{G}$. Then $Q_{1 i}$ normalizes $\widetilde{F}$ and does not centralize $J$. Let $\mathfrak{U}=\boldsymbol{A}_{\mathscr{G}}(\mathfrak{F})$. Thus, $\mathfrak{i}$ does not centralize $J$. Since $\boldsymbol{A}_{\mathscr{F}^{G}}(\mathfrak{F})$ permutes transitively $\mathfrak{F}-\langle J\rangle$, it follows that $\mathfrak{A}$ permutes $\mathfrak{F}^{*}$ transitively. Hence, $\mathfrak{U}$ is non solvable. We conclude that $\mathfrak{\Omega}_{1} \pitchfork \subseteq$.

We may assume notation is chosen so that $D_{1}$ interchanges $\coprod_{1}$ and $\mathfrak{\Omega}_{2}$. Let $\mathfrak{\Omega}_{1}=\left\langle Q_{11}, Q_{12}\right\rangle$ and set $Q_{2 i}=Q_{1 i}^{D_{1}}, F_{i}=Q_{1 i} Q_{2 i}, i=1,2$. Then set $\widetilde{F}=\left\langle D_{1}, F_{1}, F_{2}, I\right\rangle$, an elementary group of order 16 . Let $\mathfrak{U}=\boldsymbol{A}_{\mathscr{s}}(\mathfrak{F}), \mathfrak{I}^{*}=\boldsymbol{A}_{\mathfrak{\Im}}(\mathfrak{F})$. Let $\mathfrak{F}_{0}=\left\langle F_{1}, F_{2}, I\right\rangle$. We argue that $\mathfrak{F}=\boldsymbol{C}_{\mathfrak{F}}\left(\mathfrak{F}_{0}\right)$. In any case, $C_{\mathfrak{E}}\left(\mathfrak{F}_{0}\right)$ is a 2 -group, and $C_{\mathfrak{\$}}\left(\mathfrak{F}_{0}\right)=\mathfrak{F}_{0}$. Since $\mathfrak{\kappa}_{1}$ contains an element $K$ which induces an outer automorphism of both $\mathfrak{\Omega}_{1}$ and $\Omega_{2}$, it follows that $C_{\mathfrak{s}}(K)$ is contained in an abelian group of typ? $(2,4)$. This implies that no element of $\mathfrak{S} K$ centralizes any elementary subgroup of $\mathfrak{F}$ of order 8 . Hence, $\mathfrak{F}=C_{\mathfrak{S}}\left(\mathfrak{F}_{0}\right)$. Since $\mathfrak{B}$ contain $\approx$ a subgroup $\mathfrak{B}_{0}$ of order 3 which normalizes $\mathfrak{F}_{0}$, we get that $\mathfrak{B}_{0}$ normalizes $\mathfrak{F}$.

We next argue that $\mathfrak{I}$ normalizes $\mathfrak{F}$. Certainly $\mathfrak{S}$ normalizes $\mathfrak{F}$. Since $\widetilde{F}_{0}=C_{\mathfrak{5}}\left\langle D_{1}, J\right\rangle$ ), we get that $D_{2}$ normalizes $\mathfrak{F}$. Since $\mathfrak{I}=$ $\mathfrak{S}\left\langle D_{1}, D_{2}\right\rangle, \mathfrak{F} \triangleleft \mathfrak{I}$. Let $\mathfrak{N}=N(\mathfrak{F}) \supseteqq \mathfrak{I} \mathfrak{B}_{0}$. Suppose 5|| $\mathfrak{R} \mid$. Since $\mathfrak{R}$ is solvable, $\mathfrak{R}$ contains a subgroup $\mathfrak{N}_{0}$ of order 15 . Since $C(I)=\subseteq$ is a 2, 3-group, $\mathfrak{R}_{0}$ permutes $\mathfrak{F}^{\ddagger}$ transitively. Since $\mathfrak{F}=C(\mathfrak{F})$, we get $\mathfrak{F}=$ $\boldsymbol{O}_{2}(\mathfrak{R})$. But $|\mathfrak{T}: \mathfrak{F}|=2^{3}$. It is easy to check that Aut $(\mathfrak{F})$ has no such subgroups. Hence, $5 \nmid|\mathfrak{N}|$, so $\mathfrak{N}$ is a 2 , 3-group, $|\mathfrak{R}|=2^{7} \cdot 3^{a}, a=1$ or 2 , so $|\Re: \Re \cap \mathfrak{S}| \leqq 3$. Since $O_{2}(\mathfrak{R} \cap \mathfrak{S})=\mathfrak{F} \mathfrak{\mathcal { C }}$ is of index 2 in $\mathfrak{I}$, and since $\mathfrak{F} \mathfrak{F} / \mathfrak{F}$ is a chief factor of $\mathfrak{R} \cap \mathfrak{S}$, it follows that $\mathfrak{F} \mathfrak{F}=\boldsymbol{O}_{2}(\mathfrak{N})$. Hence, $\boldsymbol{Z}\left(\boldsymbol{O}_{2}(\mathfrak{R})\right)=\langle I\rangle\left\langle\mathfrak{N}, \mathfrak{R} \subseteq \subseteq\right.$, and $|\mathfrak{R}|=2^{7}$.3.

On the other hand, $\mathfrak{F} \cdot C_{\mathfrak{F}}(J) \subseteq \mathfrak{S}^{G}$, and $\mathfrak{S}^{G} \cap \mathfrak{F}=\left\langle D_{1}, I, J\right\rangle$, so that $\mathfrak{S}^{G}$ normalizes $\mathfrak{F}$. Since $\mathfrak{S}^{G} \cdot C_{\mathfrak{S}}(J)$ is a $S_{2}$-subgroup of $\mathfrak{S}^{G}$, it follows that $\mathfrak{R}$ contains a $S_{2}$-subgroup of $\varsigma^{G}$. Hence, $I$ and $J$ are 
conjugate in $\mathfrak{R}$. This is absurd, since $\langle I\rangle \triangleleft \Re$. Thus, this case does not occur.

\section{Case 2. $e \neq w$.}

By Lemma 5.8, $f \geqq w$, so that $e \geqq w$. By hypothesis, $e \neq w$, so $e \geqq w+1$. On the other hand, $\mathfrak{S}$ is of width $w$, and $\mathbb{F}$ is an elementary subgroup of $\mathfrak{F}$ of order $2^{e}$. Hence, $e \leqq w+1$, so that $e=$ $w+1, f=w-1$. Since $\mathfrak{S}$ contains an elementary subgroup of order $2^{w+1}, \mathfrak{S}$ is the central product of $w$ dihedral groups.

Case 2a. $w=2$.

Since $\mathfrak{F}$ is the central product of 2 quaternion groups, $\mathfrak{S}$ is a 2, 3-group, and $|\mathfrak{B}|=3$.

Case 2a (i). $\quad \mathfrak{S}=\mathbb{R} . \quad$ (Recall that $\mathfrak{L}=\mathscr{F} \mathfrak{B} \mathfrak{R}_{1}$.)

Here the order of $\subseteq$ is $2^{6} .3$. Since $\Re_{1}=\langle I\rangle \times \Re_{2}$, where $\Re_{2}$ is a dihedral group of order 8 , we get $\mathfrak{F}=\langle I\rangle \times \mathfrak{D}_{0}$., where $\mathfrak{D}_{0}$ is a foursubgroup of $\Re_{2}$. Choose $D \in \Re_{2}-\mathfrak{D}_{0}, D^{2}=1$. We assume without loss of generality that $D$ inverts $\mathfrak{B}=\langle B\rangle$. Let $\mathfrak{\Omega}_{1}, \mathfrak{\Omega}_{2}$ be the quaternion subgroups of $\mathfrak{S}$. There are several cases.

First, suppose $D$ normalizes both $\mathfrak{\Omega}_{1}$ and $\mathfrak{\Omega}_{2}$ and that $D$ induces outer automorphisms of $\mathfrak{\Omega}_{1}$ and $\mathfrak{\Omega}_{2}$. We can then choose generators $Q_{i 1}, Q_{i 2}$ of $\mathfrak{O}_{i}$ such that $Q_{i 1}^{D}=Q_{i 2}, i=1,2$. Hence,

$$
C_{\mathfrak{p}}(D)=\left\langle I, Q_{11} Q_{12} Q_{21} Q_{22}\right\rangle,
$$

so that $J=Q_{11} Q_{12} Q_{21} Q_{22} I^{a}$, where $a=0$ or 1 . We argue that $\Im \nsubseteq \operatorname{ccl}_{\circledast}(I)$. Suppose false. Choose $I_{1} \in \mathfrak{\Im}$. Then $I_{1}=I^{A}$ for some $A$ in $\mathbb{B}$, so that $I_{1} \in \mathfrak{\Im}(\mathfrak{S})$, yielding $I \in \mathfrak{J}\left(\mathfrak{S}^{A}\right)$. Hence, $\left(\mathfrak{S}^{A} \cap \mathfrak{S}\right) \cdot \mathfrak{S}$ is a $S_{2}$-subgroup of $\mathfrak{S}$, and $\mathfrak{S E}^{A} \cap \mathfrak{S}$ is elementary of order 8. Thus, $\left|\operatorname{ccl}_{\mathfrak{S}}\left(I_{1}\right)\right|=6$, and every element of $\mathfrak{\Im}$ commutes with some involution of $\mathfrak{S}-\mathfrak{S}$. On the other hand, if $H \in \mathfrak{S}$, then $H D$ is an involution if and only if $D$ inverts $H$, so that $H \in\left\langle Q_{11} Q_{12}, J\right\rangle$. Hence, $\mathfrak{S} D$ contains at most 8 involutions. Since $\left|\operatorname{ccl}_{\mathfrak{x}}(D)\right|=8$, and involutions of $\mathfrak{T}-\mathfrak{S}$ are conjugate to $D$ in $\mathfrak{T}$. Hence, every element of $I$ is $\mathfrak{S}_{\text {-conjugate to an }}$ element of $\langle I, J\rangle-\langle I\rangle$. This is not the case, since there are just 6 elements of $\Im$ so conjugate. Thus, in particular, we have $i(\mathbb{S}) \geqq 2$.

We have determined the isomorphism class of $\mathfrak{I}$, and it is straightforward to verify that $\mathfrak{I}$ is isomorphic to a $S_{2}$-subgroup of $M_{12}$. Since $i(\mathbb{S}) \geqq 2$, it follows from a result of Brauer and Fong [11] that $\mathbb{S} \cong$ $M_{12}$. But $M_{12}$ is not an $N$-group, since $M_{12}$ contains an involution whose centralizer $\sqrt{5}$ has order 240 , from which it follows that $\sqrt{5}$ is 
non solvable.

Next, suppose $D$ normalizes both $\mathfrak{\Omega}_{1}$ and $\mathfrak{\Omega}_{2}$, but that $D$ induces an inner automorphism of $\mathfrak{\Omega}_{2}$. Since $D$ inverts $\mathfrak{B}$, it follows that $\mathfrak{B}$ centralizes $\mathfrak{\Omega}_{2}$ and that $\langle\mathfrak{B}, D\rangle$ is faithfully represented on $\mathfrak{\Omega}_{1}$. We can therefore choose generators $Q_{11}, Q_{12}$ of $\mathfrak{\Omega}_{11}$ such that $Q_{11}^{D}=Q_{12}$, and then choose generators $Q_{21}, Q_{22}$ of $\mathfrak{Q}_{22}$ such that $Q_{21}^{D}=Q_{21}, Q_{22}^{D}=Q_{22}^{d}$, where $d= \pm 1$.

Now $J=Q_{1} Q_{2}$, where $Q_{i} \in Q_{i}-\langle I\rangle, i=1,2$. Thus, $Q_{1} Q_{2}=Q_{1}^{D}-Q_{2}^{D}$, so that $Q_{1}^{-D} Q_{1}=Q_{2}^{D} Q_{2}^{-1} \in Q_{1} \cap Q_{2}=\langle I\rangle$. Hence, $D$ fixes $\langle I\rangle Q_{1}$, so $Q_{1}=\left(Q_{11} Q_{12}\right)^{ \pm 1}$, and $Q_{1}$ is inverted by $D$. Hence, $d=-1$, and $Q_{2}=Q_{22}^{ \pm 1}$. With a suitable choice of notation, we may therefore assume that $J=Q_{11} Q_{12} Q_{22}$. Choose $E \in[F-\langle I, J\rangle$. Then $[E, D]=J$, since (5) $\mathscr{S} \mathscr{C} \mathscr{N}\left(\mathfrak{S}^{G}\right)$. But $[\mathfrak{E}, D] \subseteq \mathfrak{\Omega}_{1}$, while $J \notin \mathfrak{\Omega}_{1}$. This contradiction shows that this case does not arise.

We may now assume that $D$ interchanges $\mathfrak{\Omega}_{1}$ and $\mathfrak{\Omega}_{2}$. Let $J=$ $Q_{1} Q_{2}, Q_{i} \in \mathfrak{\Omega}_{i}-\langle I\rangle$, set $Q_{1}=Q_{11}$, and choose $Q_{12}$ such that $\left\langle Q_{11}, Q_{12}\right\rangle=$ $\mathfrak{\Omega}_{1}$. Let $Q_{2 i}=Q_{1 i}^{D}, i=1,2$, so that $\mathfrak{\Omega}_{2}=\left\langle Q_{21}, Q_{22}\right\rangle$. We now get $J=J^{D}=Q_{1}^{D} Q_{2}^{D}$, so that $Q_{2}^{D}=Q_{1}^{d}, Q_{1}^{D}=Q_{2}^{d}$, where $d= \pm 1$. Since $D$ inverts $\mathfrak{B}$, we get $C_{\mathfrak{5}}(B)=\langle I\rangle$.

Let $\mathfrak{\mho}_{0}=C_{\mathfrak{f}}(D)=\left\langle I, J, Q_{12} Q_{22}\right\rangle, \mathfrak{F}=\left\langle\mathfrak{\mho}_{0}, D\right\rangle$, so that $\mathfrak{\mho}$ is elementary of order 16. Since $[\mathfrak{S}, D] \subseteq \widetilde{F}_{0}$, it follows that $\mathfrak{\mho} \triangleleft \mathfrak{I}$. On the other hand, $\mathfrak{F} \subseteq \mathfrak{S}^{a}$, so $\mathfrak{F}$ is also normal in a $S_{2}$-subgroup of $\mathfrak{S}^{a}$. Let $\mathfrak{R}=$ $N(\mathfrak{F})$. Thus, $I$ and $J$ both lie in the center of $S_{2}$-subgroups of $\Re$, so there is $N$ in $\mathfrak{R}$ such that $J=I^{N}$, and we may assume that $G=N$. Hence, $\mathfrak{S} \cap \mathfrak{S}^{G}$ contains $\left\langle\mathfrak{F}, \Re_{1}\right\rangle$. Let $\tilde{\mathfrak{I}}$ be a $S_{2}$-subgroup of $\mathfrak{R} \cap \boldsymbol{C}(J)$ which contains $\left\langle\mathfrak{F}, \Re_{1}\right\rangle$. Then $\langle\mathfrak{I}, \tilde{\mathfrak{I}}\rangle$ normalizes $\left\langle\mathfrak{F}, \Re_{1}\right\rangle$, so that $\boldsymbol{A}_{\mathfrak{R}}(\langle I, J\rangle)=\operatorname{Aut}(\langle I, J\rangle)$. Let $\mathfrak{N}_{0}=\boldsymbol{O}_{2}(\mathfrak{N}) \supseteqq \mathfrak{F}$. If $\mathfrak{N}_{0} \supset \mathfrak{F}$, then $\mathfrak{R}_{0} \subseteq$ $\mathfrak{I} \cap \tilde{\mathfrak{I}}=\left\langle\mathfrak{F}, \Re_{1}\right\rangle$, so that $\left\langle\mathfrak{F}, \Re_{1}\right\rangle=\mathfrak{R}_{0}$, as $\left|\left\langle\Re, \Re_{1}\right\rangle: \mathfrak{F}\right|=2$. Hence, $\mathfrak{R}=\mathfrak{T}\langle R\rangle$ for some subgroup $\langle R\rangle$ of order 3 which permutes $\langle I, J\rangle$ transitively. Choose $E \in \mathbb{F}-\langle I, J\rangle$. Since $R$ fixes $\mathfrak{F} E$, there is an element $F$ of $\mathfrak{F}$ such that $R$ centralizes $F E$. Then $(F E)^{2} \in\langle I, J\rangle$, so $F E$ is an involution. The only involutions of $\mathfrak{F} E$ are in $\langle I, J\rangle E$, so we may assume that $R$ centralizes $E$, as $\langle I, J\rangle E \subset$ F. In any case, we get $\langle I, J\rangle \triangleleft \mathfrak{R}$.

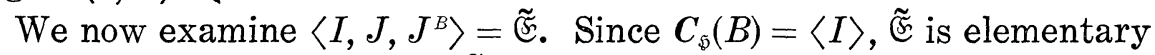
of order 8 and admits $B$. If $\tilde{F}=(F)$, then since $R$ also normalizes (F, it follows that $\boldsymbol{A}(\mathfrak{F})$ permutes ${ }^{\sharp}$ transitively. This is impossible, since $N(\mathfrak{F})$ is solvable. Hence, $\tilde{\mathfrak{F}} \neq \mathbb{F}$, so that $C_{\mathfrak{g}}(J)=\mathfrak{F} \tilde{F}$, recalling that $\Re_{1}=C_{\mathfrak{F}}(J)^{G}$, we get $\Omega_{1}=\left\langle\left(\mathfrak{F}, \widetilde{F}^{N}\right\rangle\right.$, since we have taken $N=G$, and since $\mathfrak{F} \triangleleft \mathfrak{R}$. Hence, $\mathfrak{I}-\mathfrak{F}$ contains a conjugate of $I$. As all involutions of $\mathfrak{I}-\mathfrak{S}$ are $\mathfrak{I}$-conjugate to $D$, we get $D \sim I$. Hence, $\Re$ contains a $S_{2}$-subgroup of $C(D)$, so that $D$ is in the center of a $S_{2}$-subgroup of $\mathfrak{R}$. This is not the case, $\langle I, J\rangle=\boldsymbol{Z}\left(\boldsymbol{O}_{2}(\mathfrak{R})\right)$. We conclude that $\mathfrak{F}=O_{2}(\mathfrak{R})$. 
Suppose 5|| $\mathfrak{R} \mid$. Let $\mathfrak{P}$ be a $S_{5}$-subgroup of $\mathfrak{R}$ which is permutable with $\mathfrak{T}$. Since $|\mathfrak{P}|=5$ and $\mathfrak{T} / \mathfrak{F}$ is a four-group, we have $\boldsymbol{O}_{2}(\mathfrak{T P}) \supset \mathfrak{F}$. Thus, $\boldsymbol{Z}\left(\boldsymbol{O}_{2}(\mathfrak{T} \mathfrak{P})\right) \cap \mathfrak{F}$ admits $\mathfrak{P}$. As $\mathfrak{P}$ acts irreducibly on $\mathfrak{F}$, we get that $\mathfrak{F} \subseteq Z\left(O_{2}(\mathfrak{T} P)\right)$, so that $\boldsymbol{O}_{2}(\mathfrak{P T})$ is abelian. This is not the case, since $\mathfrak{F} \in \mathscr{S} \mathscr{C} \mathscr{N}(\mathfrak{T})$. Hence, $\mathfrak{N}$ is a 2,3 -group.

Since $\mathfrak{F}=\boldsymbol{O}_{2}(\mathfrak{R})$, we see that a $S_{2^{\prime}}$-subgroup $\Re$ of $\mathfrak{R}$ is elementary of order 9 . Let $\mathfrak{E}=\operatorname{ccl}_{\mathfrak{R}}(I)$. Since $\mathfrak{R} \cap \mathfrak{S}=\mathfrak{T}$, we get that $|\mathfrak{F}|=9$, and $\Re$ permutes $\sqrt{S}$ transitively and regularly.

Let $\mathfrak{F}=\mathfrak{F}^{0} \times \mathfrak{F}^{1}$, where each $\mathfrak{F}^{i}$ is a four-group which admits $\Re$. Let $\Re_{0}=\boldsymbol{C}_{\Re}\left(\mathfrak{F}^{1}\right), \Re_{1}=\boldsymbol{O}_{\mathfrak{r}}\left(\mathfrak{F}^{0}\right), \Re_{i}=\left\langle R_{i}\right\rangle, i=0,1$. Hence,

$$
\mathfrak{F}^{\sharp}=\mathfrak{F}^{0^{\sharp}} \cup \mathfrak{F}^{1^{\sharp}} \cup \operatorname{ccl}_{\mathfrak{R}}(I) \text {. }
$$

Since $I \notin \mathfrak{F}^{0}$, we get $\mathfrak{F}^{0} \nless \mathfrak{R}$. Let $\mathfrak{U}=N_{\tilde{\Sigma}}(\mathfrak{R})$ so that $\mathfrak{U}$ is a four-group. We can choose $U_{0} \in \mathfrak{U}$ such that $U_{0}$ inverts $\mathfrak{R}$. Then choose $U_{1} \in \mathfrak{U}-$ $\left\langle U_{0}\right\rangle$. Since $U_{0}$ normalizes $\mathfrak{F}^{0}, U_{1}$ interchanges $\mathfrak{F}^{0}$ and $\mathfrak{F}^{1}$ and so interchanges $\Re_{0}$ and $\Re_{1}$. We may assume that $R_{0}^{U_{1}}=R_{1}$.

Since $\mathfrak{F}$ is weakly closed in $\mathfrak{T}$, we get that $\mathfrak{F}$ is normal in every 2-subgroup of $(\$)$ which contains $\mathfrak{F}$. (We have used this fact earlier.) Hence, $\operatorname{ccl}_{\mathfrak{R}}(I)=\operatorname{ccl}_{\mathfrak{\leftrightarrow}}(I) \cap \mathfrak{\mho}$.

Choose generators $F_{00}, F_{01}$ of $\mathfrak{F}^{0}$ such that $F_{00}^{U_{0}}=F_{01}$, and set $F_{1 i}=F_{0 i}^{U 1}, i=0,1$. Thus, $\mathfrak{F}^{1}=\left\langle F_{10}, F_{11}\right\rangle$. Hence, $\mathfrak{F}$ is a free $F_{2} \mathfrak{l}$ module, so all involutions of $\mathfrak{I}-\mathfrak{F}$ are $\mathfrak{T}$-conjugate to an element of $\mathfrak{U}$.

Suppose $U \in \mathfrak{U}-\mathfrak{H}_{0}$. Then $C_{\mathfrak{r}}(U)=\langle R\rangle$ is of order 3. Suppose also that $U \sim I$. Since $U_{1}$ and $U_{0} U_{1}$ interchange $\Re_{0}$ and $\Re_{1}$, it follows that $R \notin \Re_{0} \cup \Re_{1}$, so that $R$ has no fixed points on $\mathfrak{F}$. Let $W=C_{\S}(U)$, a four-group. Thus, $\mathfrak{u}\langle R\rangle \mathfrak{W} \subseteq C_{\mathfrak{n}}(U)$, and $I \in \mathfrak{W}$. Hence, $\mathfrak{W} \times U \subseteq$ $\boldsymbol{O}_{2}(\boldsymbol{C}(U))$. But $\boldsymbol{A}_{\Theta}(\mathfrak{W} \times\langle U\rangle)$ contains the stability group of $\mathfrak{W} \times$ $\langle U\rangle \supset\langle U\rangle \supset 1$, since $\mathfrak{W} \times\langle U\rangle \subseteq O_{2}(U)$ ), and also contains the stability group of $\mathfrak{W} \times\langle U) \supset \mathfrak{W} \supset 1$, since $\mathfrak{F}$ normalizes $\mathfrak{W} \times\langle U\rangle$. Hence, $\boldsymbol{A}_{\mathscr{\Theta}}(\mathfrak{W} \times\langle U\rangle)$ acts irreducibly on $\mathfrak{W} \times\langle U\rangle$, so is non solvable. We conclude that if $U \in \mathfrak{U}-\left\langle U_{0}\right\rangle$, then $U \nsim I$. Hence, if $V$ is any involution of $\mathfrak{I}-\mathfrak{F}\left\langle U_{0}\right\rangle$, then $V \nsim I$.

Let $Y=J^{B}$, where $\mathfrak{B}=\langle B\rangle$ is a $S_{3}$-subgroup of $\mathfrak{S}$ inverted by $D$. Then $\langle I, Y, J\rangle$ is elementary of order 8. If $\langle I, Y, J\rangle=\mathfrak{F}_{0}$, then since $D$ centralizes $\mathfrak{F}_{0}$, so does $B$. This is obviously not the case, so $\langle I, Y, J\rangle \neq \widetilde{F}_{0}$. Since $\widetilde{F}_{0}=\widetilde{F} \cap \mathfrak{S}$, we get $Y \notin \widetilde{F}$. By the previous argument, $Y \in \mathfrak{F} U_{0}$. Hence, $Y$ inverts some $S_{3}$-subgroup of $\mathfrak{R}$, so $Y$ normalizes both $\mathfrak{F}^{0}$ and $\mathfrak{F}^{1}$. Hence, $\boldsymbol{C}_{\mathfrak{F}}(Y)=\boldsymbol{C}_{\mathfrak{F}^{0}}(Y) \times \boldsymbol{C}_{\mathfrak{\mho}^{1}}(Y)$. But $\boldsymbol{C}_{\widetilde{\mho}}(Y) \supseteqq\langle I, J\rangle$, so $\langle I, J\rangle=\boldsymbol{C}_{\mathfrak{F}^{0}}(Y) \times \boldsymbol{C}_{\mathfrak{F}^{1}}(Y)$. Thus, $J \in \mathfrak{F}^{0} \cup \mathfrak{F}^{1}$. This contradiction shows that this case does not occur.

Case 2a(ii). $\mathfrak{S} \supset \mathfrak{R}$.

Here we get that $S_{2^{\prime}}$-subgroups of $\subseteq$ are elementary of order 9 . Hence, $\mathfrak{S}$ permutes $\mathfrak{\Im}$ transitively. Since $\mathfrak{S}$ has just 6 elementary 
subgroups of order 8 , there is a subgroup $\mathfrak{R}$ of $\subseteq$ of order 3 which normalizes (5. Let $A=A_{\mathbb{S}}(\mathfrak{\xi})$. Then $\langle I\rangle$ is the only proper subgroup

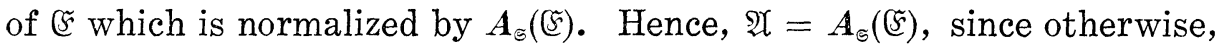
$\mathfrak{U}$ acts irreducibly on $\mathfrak{F}$ so is non solvable. But $\mathfrak{Z}$ also contains the stability group of $\mathfrak{F} \supset\langle J\rangle \supset 1$, since $\mathfrak{F} \subseteq \mathfrak{F}^{G}$. This contradiction shows that this case does not arise.

Case $2 \mathrm{~b} . \quad w=3$ and $\mathfrak{B}$ is a 3 -group.

Since $f=w-1, \mathfrak{B}$ is elementary of order 9 . Since $|\mathfrak{F}|=2^{w+1}, \mathfrak{F}$ is the central product of 3 dihedral groups. Hence, $\mathfrak{F}$ is not the central product of 3 quaternion groups.

Case $2 b(\mathrm{i}) . \quad C_{\mathfrak{5}}(\mathfrak{B}) \supset\langle I\rangle$.

Let $\Omega_{1}=C_{\mathfrak{F}}(\mathfrak{B}), \Omega_{2}=[\mathfrak{S}, \mathfrak{B}]$. Thus, $\mathfrak{B}$ is represented faithfully on $\mathfrak{\Omega}_{2}$, so that $\mathfrak{\Omega}_{2}$ is the central product of 2 quaternion groups $\mathfrak{\Omega}_{21}, \mathfrak{\Omega}_{22}$. Hence, $\mathfrak{\Omega}_{1}$ is dihedral, and $Q_{1}, Q_{2}$ admit $\Omega_{1}$.

Suppose $J \in \mathfrak{Z}_{1} \mathfrak{\Omega}_{21} \cup \mathfrak{D}_{1} \mathfrak{\Omega}_{22}$. In this case, some element $B$ of $\mathfrak{B}^{\text {t }}$ centralizes $J$, so $B \in \mathfrak{S}^{G},\left[\Re_{1}, B\right] \subseteq \mathfrak{S}^{G} \cap \mathfrak{S} \mathfrak{B} \subseteq \mathfrak{S}_{2}$. This is not the case, since $\Re_{1}$ fixes no non identity element of $\mathfrak{S} \mathfrak{B} / \mathscr{S}$.

Write $J=Q_{1} Q_{21} Q_{22}$, where $Q_{1} \in \Omega_{1}, Q_{2 i} \in \Omega_{2 i}-\langle I\rangle$. Hence, $Q_{1}^{2}=1$, since $J$ is an involution. Suppose $Q_{1} \notin\langle I\rangle$. Since $\Re_{1}$ normalizes $\mathfrak{\Omega}_{1}$, and since $\mid \Re_{1}:\left[\mathfrak{F} \mid=2^{w-1}=2^{2}\right.$, we can choose $K \in \Re_{1}-\mathfrak{F}$ such that $\left[\bigcap_{1}, K\right] \leqq\langle I\rangle$. Hence, $[\mathfrak{S}, K] \subseteq \Omega_{2}$. But then $[\mathfrak{S}, K] \subseteq\langle J\rangle \cap \mathfrak{\Omega}_{2}=1$, against $\left(\mathfrak{F} \in \mathscr{P} \mathscr{C} \mathscr{N}\left(\mathfrak{S C}^{G}\right)\right.$. Hence, $Q_{1} \in\langle I\rangle$, so we may assume that $Q_{1}=1$, after changing notation.

Suppose $\mathfrak{F} \cap \mathfrak{\Omega}_{2} \supset\langle I, J\rangle$. Choose $E \in \mathfrak{\Omega}_{2}-\langle I, J\rangle$ and set $\mathfrak{\Im}_{0}=$ $\langle I, J, E\rangle$. We can then choose $B \in \mathfrak{B}^{\sharp}$ such that $B$ normalizes $\mathfrak{E}_{0}$.

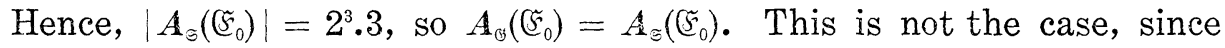
$A_{\Theta}\left(\widetilde{E}_{0}\right)$ contains the stability group of the chain $\widetilde{S}_{0} \supset\langle J\rangle \supset 1$. Hence, $\tilde{5} \cap \mathfrak{\Omega}_{2}=\langle I, J\rangle$.

Let $U_{1}, U_{2}$ be non commuting involutions of $\Omega_{1}$, so that $\Omega_{1}=$ $\left\langle U_{1}, U_{2}\right\rangle$. Since $|\tilde{r}:\langle I, J\rangle|=4$, we can choose $E_{1}, E_{2}$ in (5 such that $E_{1}=U_{1} U^{1}, E_{2}=U_{2} U^{2}$, where $U^{1}, U^{2} \in \mathfrak{\Omega}_{2}$. Since $\left[\Re_{1},[\xi]=\langle J\rangle\right.$, it follows that $\left[\Re_{1}, \mathfrak{\Omega}_{1}\right] \subseteq\langle I\rangle$. Since $U_{1}, U_{2}$ are non commuting involutions, $U^{1}, U^{2}$ are also non commuting involutions, which, however, commute with $J$. Hence,

$$
C_{\Sigma_{2}}(J)=\langle J\rangle \times\left\langle U^{1}, U^{2}\right\rangle \text {, and }\left[\left\langle U^{1}, U^{2}\right\rangle, \Re_{1}\right] \subseteq\langle I, J\rangle \text {. }
$$

On the other hand, $\Re_{1}=C_{\tilde{\xi}^{G}}(I)$, so that $\Re_{1}=\langle I\rangle \times \Re_{2}$, where $\Re_{2}$ is the central product of 2 dihedral groups. Hence, $\Re_{2}$ contains a

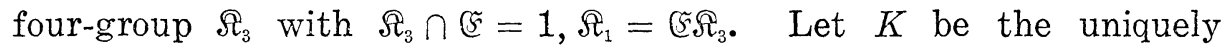
determined involution of $\Re_{3}$ such that $\mathfrak{F} K$ inverts $\mathfrak{S c} \mathfrak{B} / \mathfrak{F}$. Hence, $K$ 
inverts some $S_{3}$-subgroup of $\mathfrak{R}$, which we may assume is $\mathfrak{B}$. Hence, $K$ normalizes both $\mathfrak{\Omega}_{21}$ and $\mathfrak{\Omega}_{22}$, and induces outer automorphisms of each. Let $Q_{i 1}, Q_{i 2}$ be generators of $\mathfrak{\Omega}_{2 i}$ such that $Q_{i 1}^{K}=Q_{i 2}, i=1,2$. Hence, $C_{\mathfrak{a}_{2}}(K)=\langle I, J\rangle=\left\langle Q_{11} Q_{12} Q_{21} Q_{22}, I\right\rangle$, so that

$$
C_{\mathbb{Q}_{2}}(J)=\left\langle Q_{11} Q_{12} Q_{21} Q_{22}\right\rangle \times\left\langle Q_{11} Q_{21}, Q_{11} Q_{12} Q_{21}\right\rangle .
$$

But $\left[Q_{11} Q_{12} Q_{21}, K\right]$ is an element of $\mathfrak{\Omega}_{22}$ of order 4 , so is not in $\langle I, J\rangle$. Thus, this case does not occur.

\section{Case $2 \mathrm{~b}(\mathrm{ii}) . \quad C_{\mathfrak{F}}(\mathfrak{B})=\langle I\rangle$.}

Since $\mathfrak{B}$ acts faithfully on $\mathfrak{K}$, we can choose distinct subgroups $\mathfrak{B}_{1}, \mathfrak{B}_{2}$ of $\mathfrak{B}$ of order 3 such that $\mathfrak{N}_{i}=C\left(\mathfrak{B}_{i}\right) \supset\langle I\rangle, i=1,2$. If $\mathfrak{N}_{1} \mathfrak{\Omega}_{2} \subset \mathfrak{F}$, then $\mathfrak{\Omega}_{1}, \mathfrak{\Omega}_{2}$ are both quaternion groups, and $\mathfrak{K}$ is the central product of $\mathfrak{\Omega}_{1}, \mathfrak{\Omega}_{2}, \mathfrak{\Omega}_{3}$, where $\mathfrak{\Omega}_{3}$ is dihedral. This gives $C_{\mathfrak{5}}(\mathfrak{B})=\mathfrak{\Omega}_{3}$. Hence, $\mathfrak{S}$ is the central product of $\mathfrak{\Omega}_{1}$ and $\mathfrak{\Omega}_{2}$, and we can choose notation so that $\mathfrak{\Omega}_{1}$ is the central product of $\mathfrak{\Omega}_{11}$ and $\mathfrak{\Omega}_{12}$, where $\mathfrak{\Omega}_{11}$ is quaternion and $\mathfrak{\Omega}_{12}$ is dihedral, and $\mathfrak{\Omega}_{2}$ is quaternion. This is impossible, since we have assumed that $\boldsymbol{C}_{\mathfrak{f}}(\mathfrak{B})=\langle I\rangle$. So this case does not arise.

Case 2c. $w=3$ and $\mathfrak{B}$ is not a 3-group.

By Lemma $5.15, \mathfrak{B}=\mathfrak{B}_{1} \times \mathfrak{B}_{2}$, where $\left|\mathfrak{B}_{1}\right|=3,\left|\mathfrak{B}_{2}\right|=5$, and $\mathfrak{S}$ is the central product of $\mathfrak{\Omega}_{1}$ and $\mathfrak{\Omega}_{2}$, where $\mathfrak{\Omega}_{2}$ is a quaternion group and $A_{\mathfrak{R}}\left(\mathfrak{\Omega}_{1} / \mathfrak{\Omega}_{1}^{\prime}\right)$ is dihedral of order 10. As above, we get $J \notin \mathfrak{\Omega}_{1} \cup Q_{2}$,

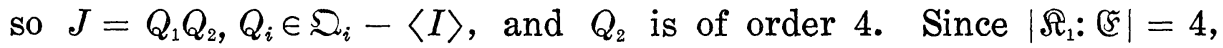
there is $K$ in $\Re_{1}-\mathfrak{F}$ such that $\left[\mathfrak{\Omega}_{2}, K\right] \subseteq\langle I\rangle$, so that $[\mathfrak{F}, K] \subseteq\langle J\rangle \cap$ $\mathfrak{\Omega}_{1}=1$, against $\mathbb{F} \in \mathscr{S} \mathscr{C} \mathscr{N}\left(\mathfrak{S}^{G}\right)$.

Case 2 d. $w \geqq 4$.

First, suppose $\mathfrak{S}$ is the central product of $\Omega_{1}$ and $\mathfrak{\Omega}_{2}$, that $\mathfrak{\Omega}_{i}$ admits $\&, i=1,2$, and in addition, the following hold:

(a) $\quad C_{\mathfrak{g}}\left(\mathfrak{\Omega}_{1}\right) \neq 1$,

(B) $\Re_{1} / \mathfrak{S}$ does not act faithfully on $\mathfrak{\Omega}_{2} /\langle I\rangle$.

Let $\mathfrak{B}_{1}=C_{\mathfrak{B}}\left(\mathfrak{\Omega}_{1}\right)$. Suppose $J \in \mathfrak{\Omega}_{1}$. Then $\mathfrak{B}_{1} \subseteq \Im^{G}$, so that $\left[\mathfrak{B}_{1}, \mathfrak{R}_{1}\right] \subseteq$ $\mathfrak{S}^{G} \cap \mathfrak{S} \mathfrak{B} \subseteq \mathfrak{S}$, against the fact that $\Re_{1}$ has no non trivial fixed points

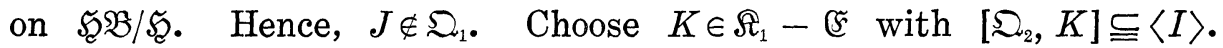
Such a choice is possible by $(\beta)$. Then $[\mathfrak{F}, K] \subseteq \mathfrak{D}_{1}$, so $[\mathfrak{F}, K] \subseteq \mathfrak{D}_{1} \cap$ $\langle J\rangle=1$, against $\mathbb{S} \in \mathscr{S} \mathscr{C} \mathscr{N}\left(\mathscr{S}^{a}\right)$. So no such $\mathfrak{\Omega}_{1}, \mathfrak{\Omega}_{2}$ exist.

For each subset $\mathfrak{A}$ of $\mathfrak{S}$, let $\overline{\mathfrak{A}}=\mathfrak{A} \mathfrak{S} / \mathfrak{S}$. Let $V=\mathfrak{S} / \mathfrak{S}^{\prime}$, and, as usual, $\mathfrak{L}=\mathfrak{S} \mathfrak{B} \mathfrak{B} \Re_{1}$.

Case $2 \mathrm{~d}(\mathrm{i}) . \quad V$ is an irreducible $\overline{\mathfrak{\Omega}}$-module. 
Since $\overline{\mathfrak{L}}$ acts faithfully on $V$, we get $2 w \equiv 0\left(\bmod 2^{w-1}\right)$. Since $w \geqq 4$, we get $w=4$. By Lemma 5.15, $|\mathfrak{B}|=3^{3}$. Let $V=V_{1} \times V_{2} \times$ $V_{3} \times V_{4}$, where each $V_{i}$ is an irreducible $\mathfrak{B}$-group. Let $\mathfrak{B}_{i}=C_{\mathfrak{B}}\left(V_{i}\right)$, $V_{i}=\mathfrak{Q}_{i} / \mathfrak{S}^{\prime}$. Thus, $\left|\mathfrak{B}: \mathfrak{B}_{i}\right|=3$ and $\left|V_{i}\right|=4$, while $\mathfrak{R}_{1}$ permutes transitively $\left\{V_{1}, V_{2}, V_{3}, V_{4}\right\}$ and $\left\{\overline{\mathfrak{B}}_{1}, \overline{\mathfrak{B}}_{2}, \overline{\mathfrak{B}}_{3}, \overline{\mathfrak{B}}_{4}\right\}$. Furthermore, $\mathfrak{O}_{i} \cong \mathfrak{Q}_{j}$ all $i, j$, and $\mathfrak{\Omega}_{1}$ is quaternion or elementary.

Suppose $\mathfrak{B}_{1}=\mathfrak{B}_{2}$. Then $\mathfrak{B}_{3}=\mathfrak{B}_{4}$, so that $\mathfrak{B}_{1} \cap \mathfrak{B}_{3} \neq 1$, and $\mathfrak{B}_{1} \cap \mathfrak{B}_{3}$ centralizes $\mathfrak{K}$. This is impossible, so $\mathfrak{B}_{i}=\mathfrak{B}_{j}$ implies $i=j$. Hence, $\mathfrak{Q}_{i}=C_{\mathfrak{p}}\left(\mathfrak{B}_{i}\right)$, so that $\mathfrak{Q}_{i}$ is quaternion and $\mathfrak{S}$ is the central product of $\mathfrak{O}_{1}, \mathfrak{\Omega}_{2}, \mathfrak{\Omega}_{3}, \mathfrak{\bigcap}_{4}$. Also, $\left|\mathfrak{B}_{i} \cap \mathfrak{B}_{j}\right|=3,1 \leqq i<j \leqq 4$.

If $J \in \mathfrak{Q}_{1} \mathfrak{\Omega}_{2}$, then $\mathfrak{B} \cap \mathfrak{S}^{G} \supset \mathfrak{B}_{1} \cap \mathfrak{B}_{2} \supset 1$, so that $\left[\mathfrak{B}_{1} \cap \mathfrak{B}_{2}\right.$, $\left.\mathfrak{R}_{1}\right] \subseteq \mathfrak{S}^{G} \cap$ $\mathfrak{B S S} \subseteq \mathfrak{F}$. This is not the case, so if $1 \leqq i<j \leqq 4$, then $J \notin \mathfrak{Q}_{i} \mathfrak{S}_{j}$. Hence, $J=Q_{1} Q_{2} Q_{3} Q_{4}, Q_{i} \in \mathfrak{S}_{i}$, and at least 3 of the $Q_{i}$ are of order 4 . Since $J^{2}=1$, we have $Q_{i} \in \mathfrak{\Omega}_{i}-\langle I\rangle$, all $i$.

Since $\Re_{1}=\langle I\rangle \times \Re_{2}$, where $\Re_{2}$ is the central product of 3 dihedral groups, $\Omega_{2}$ contains a subgroup $\Omega_{3}$ such that $\Omega_{3}$ is elementary of order 8 and $\Re_{3} \cap \mathbb{F}=1, \Re_{1}=\widetilde{F}_{3}$. Let $K$ be the uniquely determined involution of $\Omega_{3}$ such that $K$ inverts $\overline{\mathfrak{B}}$. We assume without loss of generality that $K$ inverts $\mathfrak{B}$. Thus, $\mathfrak{\Omega}_{i}$ has generators $Q_{i 1}, Q_{i 2}$ with $Q_{i 1}^{K}=Q_{i 2}$. Since $K$ centralizes $J, K$ inverts $Q_{i}, 1 \leqq i \leqq 4$. Interchanging $Q_{i 1}$ and $Q_{i 2}$ if necessary, we may assume that $Q_{i}=Q_{i 1} Q_{i 2}$. Suppose $i \neq j$. Then $J$ centralizes $Q_{i 1} Q_{j 1}$, so $\left[Q_{i 1} Q_{j 1}, K\right] \in \mathfrak{F}^{G} \cap \mathfrak{F}=\mathbb{F}$. Hence,

$$
Q_{i 1} Q_{i 2} Q_{j 1} Q_{j 2} \in \mathbb{F},
$$

all $i, j, i \neq j$.

By our construction, we have $\mathfrak{B} \subseteq \boldsymbol{F}(\subseteq \bmod \mathfrak{F})$. Let $\left(\mathfrak{C}\right.$ be a $S_{2^{\prime}}$ subgroup of $\boldsymbol{F}(\mathfrak{S} \bmod \mathfrak{S})$ which contains $\mathfrak{B}$. Since $\mathfrak{\Omega}_{i}=\boldsymbol{C}_{\mathfrak{f}}\left(\mathfrak{B}_{i}\right), \mathfrak{\Omega}_{i}$ admits $C_{\mathfrak{G}}(\mathfrak{B})$. Hence, $\boldsymbol{O}_{3^{\prime}}(\mathfrak{E})=1$, so that $\mathbb{E}$ is a 3-group. Suppose $\mathfrak{B} \subset C_{\mathscr{E}}(\mathfrak{B})$. Since $\boldsymbol{C}_{\mathfrak{g} \mathfrak{E}}(\mathfrak{B})=\boldsymbol{C}_{\mathfrak{\varpi}}(\mathfrak{B}) \times\langle I\rangle, K$ normalizes $\boldsymbol{C}_{\mathfrak{E}}(\mathfrak{B})$. If $C \in$ $\boldsymbol{C}_{\mathfrak{G}}(\mathfrak{B}) \cap \boldsymbol{C}(K)$, then $C$ normalizes $\mathfrak{Q}_{i}$ and $\left[\mathfrak{Q}_{i}, K\right]$, so $C$ centralizes each $\mathfrak{O}_{i}$, so $C=1$. Hence, $K$ inverts $\boldsymbol{C}_{\varpi}(\mathfrak{B})$. This implies that $\boldsymbol{C}_{\varpi}(\mathfrak{B})$ is elementary of order $3^{4}$. But in this case

$$
\boldsymbol{C}_{\mathbb{E}}(\mathfrak{B}) \cap \boldsymbol{C}\left(Q_{11} Q_{12} Q_{21} Q_{22}\right) \in \mathscr{I}^{*}(\mathfrak{S}),
$$

so that $C\left(Q_{11} Q_{12} Q_{21} Q_{22} I^{a}\right) \subseteq \subseteq$ for $a=0$ and 1. This implies that $\mathfrak{F}^{\alpha} \subset \mathfrak{S}$, which is not the case. Hence, $\mathfrak{B} \in \mathscr{S} \mathscr{C} \mathscr{N}^{-}(\mathfrak{C})$. Suppose $\mathfrak{B} \subset \mathbb{C}$. Then $\mathfrak{C} / \mathfrak{B}$ is faithfully represented as permutations of $\left\{\mathfrak{\Omega}_{1}, \mathfrak{\Omega}_{2}, \mathfrak{\Omega}_{3}, \mathfrak{\Omega}_{4}\right\}$, since if $\widetilde{\mathbb{E}}=\bigcap_{i=1}^{4} \mathbb{E} \cap N\left(\mathfrak{\Omega}_{i}\right)$, then $[\widetilde{\mathfrak{S}}, \mathfrak{B}]$ centralizes $\mathfrak{K}$, so that $[\widetilde{\mathfrak{E}}, \mathfrak{B}]=1$, $\widetilde{\mathfrak{C}}=\mathfrak{B}$. Hence, $|\mathfrak{S}: \mathfrak{B}|=3$. Suppose $|\boldsymbol{Z}(\mathfrak{C})|=3^{2}$. Choose $C \in Z(\mathfrak{C})^{\#}$ such that $\boldsymbol{C}_{\mathfrak{\xi}}(C) \supset\langle I\rangle$. Then $\boldsymbol{C}_{\mathfrak{\wp}}(C)$ admits $\mathfrak{E}$, so $\mathbb{C}^{\prime}$ centralizes $\boldsymbol{C}_{\mathfrak{5}}(C)$, as $\boldsymbol{C}_{\mathfrak{5}}(C)$ has width at most 2 . Since $\mathfrak{\mathcal { E }}=\left\langle\boldsymbol{C}_{\mathfrak{5}}(C) \mid C \in \boldsymbol{Z}(\mathfrak{E})^{\sharp}\right\rangle$, we get

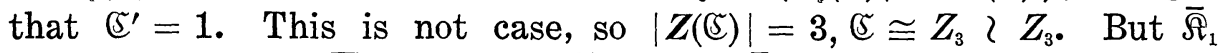
acts faithfully on $\overline{\mathfrak{E}}$, against $m(\overline{\mathfrak{E}})=2, m\left(\bar{\Re}_{1}\right)=3$. Hence, $\mathfrak{B}=\mathfrak{C}$, so 
that $F(\mathfrak{S} \bmod \mathfrak{S})=\mathfrak{S} \mathfrak{B}$.

It is a direct consequence of the preceding equality that $\subseteq$ is a 2,3-group and that $S_{3}$-subgroups of $\subseteq$ have order $3^{3}$ or $3^{4}$.

We next show that if $\mathfrak{X}$ is any non identity 2-subgroup of $\mathbb{B}$, then $N(\mathfrak{X})$ is a 2,3-group. Suppose $p \geqq 5$ and $p|| N(\mathfrak{X}) \mid$. By Lemma 13.1, $\boldsymbol{O}_{2^{\prime}}(N(\mathfrak{X}))=1$. Thus, $p|| \boldsymbol{A}_{\circledast}(\mathfrak{Y}) \mid$, where $\mathfrak{Y}=\boldsymbol{O}_{2}(N(\mathfrak{X}))$. By Lemma 5.51, $\mathfrak{I}$ contains a normal subgroup $\mathfrak{I}_{0}$ snch that $p|| A_{\Theta}\left(\mathfrak{I}_{0}\right) \mid$. Since $\mathfrak{I} \cong N\left(\mathfrak{I}_{0}\right), N\left(\mathfrak{T}_{0}\right)$ contains an elementary $p$-subgroup $p \neq 1$ which is permutable with $\mathfrak{T}$. Let $\mathfrak{M}=\mathfrak{T} \mathfrak{P}, \mathfrak{I}_{1}=\boldsymbol{O}_{2}(\mathfrak{M})$. If $\mathfrak{S}_{\cong} \subseteq \mathfrak{I}_{1}$, then $I$ is the central involution of $\mathfrak{I}_{1}$, so that $\mathfrak{M} \subseteq \mathfrak{S}$. This is not the case, since $\mathfrak{S}$ is a 2,3-group. Hence, $\mathfrak{S}_{\mathbb{E}} \nsubseteq \mathfrak{I}_{1}$, but of course $I \in \mathfrak{I}_{1}$. Since $\mathfrak{I}_{1} \mathfrak{S}_{\mathrm{C}} / \mathfrak{T}_{1} \cong \mathfrak{S} / \mathfrak{S}_{\mathrm{C}} \cap \mathfrak{I}_{1}$ is elementary, $\mathfrak{P}$ contains a subgroup $\mathfrak{P}_{0}$ of order $p$ which is permutable with $\mathfrak{I}_{1} \mathfrak{S}_{\text {. }}$ Let $\mathfrak{I}_{2}=\boldsymbol{O}_{2}\left(\mathfrak{I}_{1} \mathfrak{S}_{\mathfrak{C}} \mathfrak{P}_{0}\right)$, so that

$$
\left|\mathfrak{I}_{1} \mathfrak{S}_{\mathrm{C}}: \mathfrak{I}_{2}\right| \leqq 2, \quad\left|\mathfrak{S}_{\mathcal{2}}: \mathfrak{S} \cap \mathfrak{I}_{2}\right| \leqq 2 .
$$

By Lemma 13.62, $\Omega_{1}\left(\boldsymbol{Z}\left(\mathfrak{T}_{2}\right)\right)$ has order at most 4 , so is centralized by $\mathfrak{P}_{0}$, as $p \geqq 5$. Hence, $\mathfrak{P}_{0} \subseteq \subseteq$. This contradiction establishes the assertion.

Set $E=Q_{11} Q_{12} Q_{21} Q_{22} \in \mathfrak{F}$. We will show that $C(E) \subseteq \mathfrak{S}$. Let $\mathbb{F}=$ $C(E)$, a 2, 3-group. Also, $\mathfrak{B}_{1} \cap \mathfrak{B}_{2}=\tilde{\mathfrak{B}}$ is contained in $\mathfrak{C}$, and is of order 3. We first show that $\tilde{\mathfrak{B}}$ is a $S_{3}$-subgroup of $\sqrt{5}$. Namely, $\mathfrak{B} \subseteq C(\tilde{\mathfrak{B}})$, so that $\boldsymbol{C}_{\mathscr{E}}(\tilde{\mathfrak{B}}) \in \mathscr{C}^{*}(\mathfrak{S})$, and so $C(\tilde{\mathfrak{B}}) \subseteq \mathfrak{S}$. Suppose $X$ is a 3-element in $\left(\mathfrak{S} \cap \boldsymbol{C}(\tilde{\mathfrak{B}})\right.$. Then $X$ normalizes $\mathfrak{S} \cap \boldsymbol{C}(\mathfrak{B})=\mathfrak{\Omega}_{1} \mathfrak{\Omega}_{2}$, since $X \in \mathfrak{S}$. Since $\mathfrak{N}_{1}, \mathfrak{N}_{2}$ are the only quaternion subgroup of $\mathfrak{\Omega}_{1} \mathfrak{Q}_{2}, X$ normalizes $\mathfrak{D}_{1}$ and $\mathfrak{\Omega}_{2}$. Since $X$ centralizes $Q_{11} Q_{12} Q_{21} Q_{22}, X$ centralizes $\mathfrak{O}_{1} \mathfrak{N}_{2}$. If $\langle\tilde{\mathfrak{B}}, X\rangle$ is non cyclic, then there is $Y \in\langle\tilde{\mathfrak{B}}, X\rangle^{\#}$ such that $\boldsymbol{C}_{\mathfrak{\xi}}(Y) \supset \mathfrak{\Omega}_{1} \mathfrak{\Omega}_{2}$. Thus, $\boldsymbol{C}_{\mathfrak{5}}(Y)=\mathfrak{D}_{1} \mathfrak{N}_{2} \mathfrak{D}_{i}$ for some $i \in\{3,4\}$. However, $V$ is an irreducible \&-group, and so $Y$ centralizes $V$, yielding $Y=1$. We conclude that $\tilde{\mathfrak{B}}$ is a $S_{3}$-subgroup of $\mathbb{C} \cap C(\tilde{\mathfrak{B}})$, so $\tilde{\mathfrak{B}}$ is a $S_{3}$-subgroup of $\mathfrak{C}$. Let $\tilde{\mathfrak{I}}$ be a $S_{2}$-subgroup of $\mathbb{C} \cap \mathfrak{S}$, and let $\mathfrak{I}^{*}$ be a $S_{2}$ subgroup of $\mathbb{F}$ which contains $\tilde{\mathfrak{I}}$. Let $\mathfrak{I}^{* *}$ be a $S_{2}$-subgroup of $\mathbb{B}$ which contains $\mathfrak{T}^{*}$, and let $I^{*}$ be the central involution of $\mathfrak{T}^{* *}$. If $I^{*}=I$, then $\tilde{\mathfrak{I}}=\mathfrak{I}^{*}$, so that $\mathfrak{C} \subseteq \mathfrak{S}$. Suppose $I^{*} \neq I$. Then $I^{*}$ centralizes $\mathfrak{S} \cap \mathfrak{S}=C_{\mathfrak{\$}}(E)$, so by Lemma $13.62, I^{*} \in\langle E, I\rangle$. Thus, $I^{*}=E$ or $E I$. In any case, we have $E=I^{Y}$ for some $Y$ in $\mathbb{S}$, since $E \widetilde{F} E I$. Hence, $\tilde{\mathfrak{B}} \subseteq \Im^{Y}$. Since $C(\tilde{\mathfrak{B}}) \subseteq \subseteq$, we get that $S_{2}$-subgroups of $\Im \cap \mathfrak{S}^{Y}$ are non cyclic, against Theorem 13.1. Hence, $\mathfrak{c} \subseteq \subseteq$. Since $E \widetilde{s} E I$, we also have $\boldsymbol{C}(E I) \subseteq \subseteq$. Hence, $\mathfrak{S}^{G}=\left\langle\boldsymbol{C}_{\mathfrak{F}^{G}}(E), \boldsymbol{C}_{\mathfrak{5} G}(E I), \boldsymbol{C}_{\mathfrak{\S} G}(I)\right\rangle \subseteq \subseteq$, so that $\mathfrak{S C}_{\mathcal{C}} \mathcal{C}^{G}$ is a 2-subgroup of $\subseteq$ whose center is contained in $\langle I\rangle \cap$ $\langle J\rangle=1$. This contradiction shows that case does not occur.

Case $2 \mathrm{~d}$ (ii). $\quad V$ is a reducible $\bar{\Omega}$-module and $\bar{\Omega}$ acts faithfully on no proper submodule of $V$. 
By Lemma 5.7, $V$ is completely reducible. Let $V=V_{1} \times V_{2}$, where $V_{i} \neq 1$, and $V_{2}$ is irreducible. Let $\&_{2}$ be the subgroup of $\mathbb{L}$ which contains $\mathfrak{S}$ and satisfies $\mathfrak{R}_{2}=\boldsymbol{C}_{\bar{\varepsilon}}\left(V_{2}\right)$. Thus, $\mathfrak{S} \subset \mathfrak{R}_{2} \triangleleft \mathfrak{R}$, so $\mathfrak{Q}_{2} \cap \mathfrak{B} \neq 1$. Let $\mathfrak{\Omega}_{1}=\left[\mathfrak{R}_{2}, \mathfrak{S}\right], \mathfrak{\Omega}_{2}=C_{\mathfrak{S}}\left(\mathfrak{Q}_{2} \cap \mathfrak{B}\right)$. Then $\mathfrak{\cap}_{i} / \mathfrak{S}^{\prime}=V_{i}, i=$ 1, 2. Since $C_{\bar{\ell}}\left(V_{2}\right) \neq 1$, and since $V_{2}$ is irreducible, it follows that $\Omega_{1}$ does not act faithfully on $V_{1}$. Thus, $(\alpha),(\beta)$ hold. This contradiction shows that this case does not occur.

Case $2 \mathrm{~d}$ (iii). $\quad V$ is a reducible $\bar{\Omega}$-module and $\bar{\Omega}$ acts faithfully on some proper submodule of $V$.

Let $W$ be a submodule of $V$ minimal subject to $C_{\bar{\varepsilon}}(W)=1$. By hypothesis, $W \subset V$. Suppose $|V: W|=2$. Then $W=\tilde{\mathfrak{F}} / \mathfrak{S C}^{\prime},|Z(\tilde{\mathcal{S}})|=4$, and $\tilde{\mathfrak{F}}=Z\left(\tilde{\tilde{F}_{c}}\right) \tilde{\mathscr{F}}_{1}$, where $\tilde{\mathfrak{F}}_{1}$ is extra special of width $w-1$. Hence, $I \in[\mathfrak{B}, \tilde{\mathfrak{S}}] \subset \tilde{\mathfrak{H}}$, and $\overline{\mathfrak{L}}$ acts faithfully on $[\mathfrak{B}, \tilde{\mathfrak{S}}] / \mathscr{S}^{\prime} \subset W$. Hence, we have $|V: W| \geqq 4$.

By Lemma 5.8, we get $|W|=2^{2(w-1)}$, and $W$ is completely reducible. Let $W=\tilde{\mathfrak{F}} / \mathscr{H}^{\prime}$. First, suppose $\tilde{\mathfrak{F}}$ is extra special. Then let $\mathfrak{S}_{1}=\boldsymbol{C}_{\mathfrak{F}}(\tilde{\mathfrak{F}})$, so that $\mathfrak{S}_{1}$ has width 1 and admits 2 . Hence, $V$ is completely reducible. If $W$ is reducible, we get $\mathfrak{U}_{1}, \mathfrak{\Omega}_{2}$ satisfying $(\alpha),(\beta)$. If $W$ is irreducible then $|W| \leqq 2^{4}$, by Lemma 5.8 , so that $w \leqq 3$. This is not the case.

It remains to treat the case where $\tilde{\mathcal{F}}$ is not extra special. Let $\mathcal{Z}=Z(\tilde{\mathscr{S}}) \supset\langle I\rangle$. Since $\bar{\Omega}$ has no fixed points on $W$, we get $|\mathcal{Z}:\langle I\rangle| \geqq 4$. Since $|V: W|=4$, we get $|3|=8$, and since 3 is an abelian group of order 8 admitting a non trivial automorphism of odd order, 3 is elementary. Since $W$ is completely reducible, $W=3 /\langle I\rangle \times \tilde{\mathfrak{B}}$, with $\widetilde{W}=$ $\mathfrak{Q} / \mathfrak{S}^{\prime}$, where $\mathfrak{Q}$ is extra special and admits $\mathfrak{L}$. Let $\widetilde{\mathfrak{Q}}=\boldsymbol{C}_{\mathfrak{\mathfrak { F }}}(\mathfrak{\Omega})$, so that $\mathfrak{\mathcal { E }}$ is the central product of $\mathfrak{Q}$ and $\tilde{\mathfrak{Q}}$ and $\mathfrak{Q}$ admits $\mathfrak{Q}$. By construction, the width of $\tilde{\mathfrak{Q}}$ is 2 , so that the width of $\mathfrak{Q}$ is $w-2 \geqq 2$. Thus, $\mathfrak{B}$ does not act faithfully on either $\tilde{\mathfrak{Q}}$ or $\mathscr{Q}$, and $\mathscr{R}_{1} / \mathbb{\&}$ does not act faithfully on $\tilde{\mathfrak{Q}} / \mathfrak{S}^{\prime}$. Thus, the pair $\mathfrak{D}, \tilde{\mathfrak{Q}}$ satisfy $(\alpha),(\beta)$. This contradiction completes the proof of this lemma.

Lemma 13.64. $\mathfrak{S} \in \mathscr{C}^{*}(\mathbb{S})$.

Proof. Suppose false. Choose $\mathfrak{R} \in \mathscr{S} \mathscr{O} \mathscr{L}(\mathbb{S})$ such that $\mathfrak{S} \subseteq \mathfrak{N} \nsubseteq \mathfrak{S}$, and with this restriction, minimize $|\mathfrak{N}|$. Let $\mathfrak{R}_{2}$ be a $S_{2}$-subgroup of $\mathfrak{N}$ which contains $\mathfrak{S}$. Then $I$ is the central involution of $\mathfrak{R}_{2}$, so $\mathfrak{N}_{2} \subseteq \mathfrak{S}$. Hence, $\mathfrak{N}=\mathfrak{R}_{2} \mathfrak{N}$, where $\mathfrak{D}$ is a $q$-group for some odd prime $q$. Since $\mathfrak{S}$ contains an element $\mathfrak{U}$ of $\mathscr{U}(2)$, we get $\boldsymbol{O}_{2}(\mathfrak{R}) \neq 1$, so $I \in O_{2}(\mathfrak{N})$.

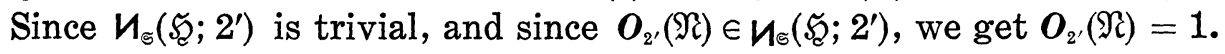

Since $I \in \boldsymbol{O}_{2}(\mathfrak{N})$, we get that $\mathfrak{S}_{2} \boldsymbol{O}_{2}(\mathfrak{N}) / \boldsymbol{O}_{2}(\mathfrak{N})$ is elementary. By minimality of $\mathfrak{R}$, we get $\mathfrak{R}=\boldsymbol{O}_{2}(\mathfrak{N}) \mathfrak{O S}$, and $\left|\mathfrak{S}: \mathfrak{S} \cap O_{2}(\mathfrak{R})\right| \leqq 2$. If $\mathfrak{S} \subseteq$ $\boldsymbol{O}_{2}(\mathfrak{R})$, then $I$ is the central involution of $\boldsymbol{O}_{2}(\mathfrak{N})$, so that $\mathfrak{N} \subseteq S_{\text {. Hence, }}$ 
$\left|\mathfrak{S}: \mathfrak{S} \cap O_{2}(\mathfrak{N})\right|=2$. Choose $Q \in \mathfrak{\Omega}-\mathfrak{S}$. Then $\left(\mathfrak{S} \cap O_{2}(\mathfrak{R})\right)^{Q} \subseteq O_{2}(\mathfrak{R}) \subseteq$ $\mathfrak{N}_{2} \subseteq \mathfrak{S}$, against Lemma 13.63. The proof is complete.

LEMMA 13.65.

(a) $\langle I\rangle$ is weakly closed in $\mathfrak{S}$.

(b) $w \geqq 3$.

Proof. Suppose $I^{G}=J \in \mathfrak{S}_{2}, G \in \mathbb{B}-\mathfrak{S}$. Let $\mathfrak{S}_{1}=C_{\mathfrak{9}}(J)$. Then $\mathfrak{S}_{1} \subseteq \mathfrak{S}^{G}=C(J)$, so $\mathfrak{S}_{1}^{G^{-1}} \subseteq \mathfrak{S}$. Since $G^{-1} \in \mathbb{S}-\mathfrak{S}$, Lemma 13.63 gives a contradiction. This establishes (a).

Suppose $w=2$. By (a) and the Glauberman $Z^{*}$-theorem [16], $\mathfrak{S} \subset \mathfrak{T}$.

Case b (i). $|\mathfrak{T}: \mathfrak{S}|=2$.

Choose $G \in \mathbb{B}-\mathfrak{S}$ such that $J=I^{G} \in \mathfrak{T}$. By (a), we get $\mathfrak{T}=\mathscr{S}\langle J\rangle$. Let $\mathbb{F}=\boldsymbol{C}_{\mathfrak{\xi}}(J) \subseteq \mathfrak{S}^{a}$. If $\mathbb{F}$ is not elementary, then $I \in D(\mathfrak{F}) \subseteq \mathcal{F}_{\mathcal{E}}$, against (a). Hence, $\widetilde{F}$ is elementary.

Suppose $|\mathbb{F}|=4$. Then $A_{\mathfrak{x}}(\langle\mathbb{F}, J\rangle)$ is the stability group of the chain $\langle\mathfrak{F}, J\rangle \supset \mathfrak{F} \supset 1$. Let $\mathfrak{F}=\langle\mathfrak{F}, J\rangle \subset \mathfrak{S}^{G}$. Let $\mathfrak{F}_{0}=\mathfrak{F} \cap \mathfrak{S}^{G}$, so that $I \notin \mathfrak{F}_{0}$, and $\boldsymbol{A}_{\widetilde{\Phi}}(\mathfrak{F})$ is the stability group of $\mathfrak{F} \supset \mathfrak{F}_{0} \supset 1$. Let $\mathfrak{F}_{1}=\mathfrak{F}_{\cap} \cap \mathfrak{F}_{0}$. Hence, $\left|\mathfrak{F}_{1}\right|=2$ and $\left\langle N_{\widetilde{E}}(\mathfrak{F}), N_{\widetilde{F}}(\mathfrak{F})\right\rangle$ maps onto the subgroup of Aut (予) which fixes $\xi_{1}$. Hence, $\mathfrak{F}_{1}$ is contained in the center of a $S_{2}$-subgroup of $\mathbb{B}, \mathfrak{F}_{1}=\langle E\rangle$, with $E \sim I$. This violates (a).

Suppose $|\mathfrak{F}|=8$. Let $\mathfrak{F}=\langle\mathfrak{F}, J\rangle$. Thus, $\operatorname{ccl}_{\mathfrak{x}}(J)$ has four elements. Let $\widetilde{\mathfrak{I}}$ be a $S_{2}$-subgroup of $\mathfrak{S}^{G}$ which contains $\widetilde{\mho}$. Then $\left|\operatorname{ccl}_{\mathfrak{x}}(I)\right|=4$. Let $\mathfrak{U}=\operatorname{ccl}_{\mathfrak{B}}(I) \cap \mathfrak{F}$, so that $\mathfrak{U} \supseteqq \operatorname{ccl}_{\mathfrak{x}}(J) \cup\{I\}$ and $\mathfrak{u} \cap \mathfrak{F}=\{I\}$. Suppose $\mathfrak{U} \supset \operatorname{ccl}_{\mathfrak{x}}(J) \cup\{I\}, U \in \mathfrak{U}-\left(\operatorname{ccl}_{\mathfrak{x}}(J) \cup\{I\}\right)$. Then $U \notin \mathfrak{F}$, so $\left|\operatorname{ccl}_{\mathfrak{x}}(\mathfrak{H})\right|=$ 4 , and so $|\mathfrak{u}| \geqq 9$. Hence, $\mathfrak{U}=\operatorname{ccl}_{\mathfrak{x}}(J) \cup \operatorname{ccl}_{\mathfrak{x}}(U) \cup\{I\}$, since $\mathfrak{U} \cap \mathfrak{F}=\{I\}$; this yields $|\mathfrak{u}|=9$. Hence, $\mathfrak{u}=\mathfrak{F} J \cup\{I\}$. Let $\mathfrak{F}^{*}=\mathfrak{F} \cap \mathfrak{S}^{G}$, so that by symmetry $\mathfrak{U}=\mathfrak{F}^{*} I \cup\{J\}$. Let $\mathfrak{U}_{0}=\mathfrak{H}-\{I, J\}$. If $U_{1}, U_{2} \in \mathfrak{U}_{0}$, then $U_{1} U_{2} \in \mathfrak{F} \cap \mathfrak{F}^{*}$, since $U_{i} \in \mathfrak{F} J \cap \mathfrak{F}^{*} I$. Hence, $\mathfrak{F}=\mathfrak{F}^{*}$, since $\left|\mathfrak{U}_{0}\right|=7$. This is false, since $I \in \mathfrak{F}, I \notin \mathfrak{F}^{*}$. We conclude that $\mathfrak{U}=\operatorname{ccl}_{\mathfrak{x}}(J) \cup\{I\}=$ $\operatorname{ccl}_{\tilde{\mathfrak{x}}}(I) \cup\{J\}$, so that $|\mathfrak{U}|=5$. Since each element of $\mathfrak{U}$ is in the center of a $S_{2}$-subgroup of $N(\mathfrak{F})$, it follows that $N(\mathfrak{F})$ permutes $\mathfrak{u}$ transitively. Hence, 5|| $\boldsymbol{A}_{\Theta}(\mathfrak{\mho}) \mid$. Let $\mathfrak{P}$ be a $S_{5}$-subgroup of $N(\mathfrak{F})$ permutable with $\mathfrak{T}$. Since $|\mathfrak{P}|=5$ and $\mathfrak{I} / \mathfrak{F}$ is elementary of order 4 , we get $\boldsymbol{O}_{2}(\mathfrak{T} \mathfrak{P}) \supset \mathfrak{F}$. Hence, $1 \subset \boldsymbol{Z}\left(\boldsymbol{O}_{2}(\mathfrak{T} \mathfrak{P})\right) \subset \mathfrak{F}$, so that $\mathfrak{P}$ centralizes $\boldsymbol{Z}\left(\boldsymbol{O}_{2}(\mathfrak{T} \mathfrak{P})\right)$, and so $\mathfrak{P}$ centralizes $\mathfrak{F}$. This is not the case, since $2 \in \pi_{4}$.

Case b (ii). $|\mathfrak{T}: \mathfrak{S}|>2$.

Since $\mathscr{S} \mathscr{C} \mathscr{N}_{3}(\mathfrak{T}) \neq \varnothing$, it follows as in an earlier argument that $\mathfrak{I} / \mathfrak{S}$ is a four-group and that $\mathfrak{S}=\mathfrak{T} \mathfrak{B}$, where $\mathfrak{B}$ is elementary of 
order $3^{2}$. By the $Z^{*}$-theorem, there is $G$ in $(S)-\subseteq$ such that $J=$ $I^{G} \in \mathfrak{T}$. By (a), $J \notin \mathfrak{S}$. Let $\mathfrak{F}=C_{\mathfrak{\xi}}(J)$. If $\mathfrak{F}$ is not elementary, then $I \in \boldsymbol{D}(\mathfrak{F}) \subseteq \mathfrak{S}_{\mathcal{C}}$, against (a). Hence, $(\mathfrak{S}$ is elementary.

Suppose $|\mathfrak{F}|=4$. Let $\mathfrak{F}=\langle\mathfrak{F}, J\rangle$. Then $\boldsymbol{A}_{\mathscr{G}}(\mathfrak{F})$ contains $\boldsymbol{A}(\mathscr{C})$ and $\boldsymbol{A}\left(\mathscr{C}^{*}\right)$, where $\mathscr{C}: \mathfrak{F} \supset \widetilde{\Im} \supset 1, \mathscr{C}^{*}: \mathfrak{F} \supset \mathfrak{F}^{*} \supset 1$, $\mathfrak{F}^{*}=\mathfrak{F} \cap \mathfrak{S}^{G}$. Since $\mathscr{C} \neq \mathscr{C}^{*}$, it follows that $\boldsymbol{A}_{\mathfrak{H}}(\mathfrak{F})$ is the subgroup of Aut $(\mathfrak{F})$ fixing ⿷匚 $\cap \mathfrak{F}^{*}=\langle E\rangle$. Thus, if $\tilde{\mathfrak{I}}$ is a $S_{2}$-subgroup of $N(\mathfrak{F})$, then $\langle E\rangle=Z(\tilde{\mathfrak{I}})$, so that $E \sim I$, against (a).

Suppose $|\mathfrak{F}|=8$. Let $\mathfrak{F}=\langle\mathfrak{F}, J\rangle$. Then $\mathfrak{F}$ normalizes $\mathfrak{F}$. Since

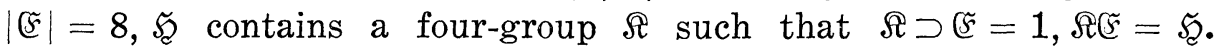
Since $\mathfrak{F}=\langle J\rangle^{\Re}, \mathfrak{F}$ is a free $F_{2} \Re$-module, so $\mathfrak{F}$ is the only subgroup of $\mathfrak{F} \mathfrak{F}$ which is isomorphic to $\mathfrak{F}$. Hence, $\mathfrak{F} \triangleleft \mathfrak{I},|\mathfrak{I}: \mathfrak{F}|=8$.

Let $\mathfrak{U}=\operatorname{ccl}_{\mathfrak{E}}(I) \cap \mathfrak{F}$, so that $|\mathfrak{u}|=5$ or 9 . If $|\mathfrak{u}|=9$, then $\mathfrak{U}=$ $\mathfrak{S} J \cup\{I\}=\mathfrak{F}^{*} I \cup\{J\}$, where $\mathfrak{F}^{*}=\mathfrak{F} \cap \mathfrak{S}^{G}$. As above, we get $\mathfrak{F}=\mathfrak{F}^{*}$, so that $J \in \mathfrak{F} \subseteq \mathfrak{F}$, against (a). Hence, $|\mathfrak{U}|=5$. Hence, $\boldsymbol{A}_{\Theta}(\mathfrak{F})$ is a multiple of $2^{3} .5$, and $\boldsymbol{O}_{2}\left(\boldsymbol{A}_{\oplus}(\mathfrak{F})\right)=1$. This forces $\boldsymbol{A}_{\circledast}(\mathfrak{F})$ to be non solvable. The proof is complete.

Lemma 13.66. Suppose $G \in(B)-\subseteq$. Then one of the following holds:

(a) $\left|\mathfrak{S}^{G}: \mathfrak{S}^{G} \cap \mathfrak{S}\right|>4$,

(b) $\left|\mathfrak{S}: \mathfrak{S} \cap \mathfrak{S}^{G}\right|>4$,

(c) $\mathfrak{S} \cap \mathfrak{S}^{G}$ does not normalize $\mathfrak{S}^{G} \cap \mathfrak{S}$,

(d) $\mathfrak{S C}^{G} \cap \mathfrak{S}$ does not normalize $\mathfrak{S E} \cap \mathfrak{S}^{G}$.

Proof. Suppose false. Let $\mathfrak{K}_{1}=\mathfrak{K} \cap \mathfrak{S}^{a}, \Re_{1}=\mathfrak{S C}^{a} \cap \Subset$. By Lemma 13.63, we have $\left|\mathfrak{S}_{c}: \mathfrak{S}_{1}\right|=\left|\mathfrak{S}^{G}: \Re_{1}\right|=4$. By Lemma 13.65, $\mathfrak{S}_{1}$ and $\Re_{1}$ are non abelian, and Lemma $13.63, \mathfrak{S}_{1} \cap \Re_{1}$ is centralized by both $\mathfrak{S}_{1}$ and $\Re_{1}$ and is elementary. Since $J \notin \mathscr{S}_{1} \cap \Re_{1}$, and $\mathscr{S}_{1} \cap \Re_{1} \triangleleft \Re_{1}$, we get that $\Re_{1}=\mathfrak{K}_{1} \cap \Re_{1} \times \Re_{2}$ for some subgroup $\Re_{2}$ of $\Re_{1}$ which is non abelian. Notice that $\mathfrak{S}_{\mathfrak{Z}} \cap \Re_{1} \subseteq \mathfrak{F}_{\mathfrak{C}} \cap \mathfrak{S}^{G}=\mathfrak{K}_{1}, \mathfrak{S} \cap \Re_{1} \subseteq \Re_{1}$, so that $\mathfrak{K}_{2} \cap \Re_{1}=\mathfrak{K}_{1} \cap \Re_{1}$.

Since $\left|\mathfrak{S}^{G}: \Re_{1}\right|=4$, we have $\left|Z\left(\Re_{1}\right)\right| \leqq 8$. Since $\left\langle\mathfrak{C}_{1} \cap \Re_{1}, J\right\rangle \subseteq Z\left(\Re_{1}\right)$ we have $\left|\mathfrak{F}_{1} \cap \Re_{1}\right| \leqq 4$. Since $\Re_{2}$ is non abelian, we can choose $K$ in $\Re_{2}$ such that $K^{2}=J$.

Let $V=\mathfrak{S}_{\mathfrak{C}} / \mathfrak{S}^{\prime}, V_{0}=\mathfrak{S}_{1} / \mathfrak{S}^{\prime}$, so that $\left|V: V_{0}\right|=4$ and $J$ centralizes $V_{0}$.

Since $\left|\mathscr{S}^{G}: \Re_{1}\right|=4, \Re_{2}$ contains a subgroup $\Re_{3}$ which is extra special of width $w-2$. Let $\mathfrak{B}$ be a $q$-subgroup of $\boldsymbol{O}_{2,2^{\prime}}(\mathfrak{S}), q$ an odd prime such that (a) $\Omega_{3}$ acts faithfully and irreducibly on $\mathfrak{S} \mathfrak{B} / \mathfrak{S} D(\mathfrak{B})$, (b) $\Omega_{3}^{\prime}$ centralizes $\mathfrak{S} \boldsymbol{D}(\mathfrak{B}) / \mathfrak{F}$, (c) $\boldsymbol{D}(\mathfrak{B}) \subseteq \boldsymbol{Z}(\mathfrak{B}),(\mathrm{d}) \mathfrak{B}$ is of exponent $q$. We are guaranteed that $\mathfrak{B}$ exists since $\Re_{3} \cap \mathfrak{S}=1$. Since the absolutely irreducible faithful representations of $\Omega_{3}$ over all fields of odd characteristic have degree $2^{w-2}$, we get $m(\mathfrak{B}) \geqq 2^{w-2}$. By Lemma 5.3 applied to $\mathfrak{B}$ acting on $V$, we get $m(V) \geqq 2^{w-1}$. Hence, $2 w=m(V) \geqq 2^{w-1}$, 
so that $w \leqq 4$.

Suppose $w=4$. Then Lemma 5.3 gives $q=3$ and forces $\mathfrak{B}$ to be elementary. In this case $J$ inverts $\mathscr{S} \mathfrak{B} / \mathfrak{S}$, so that $V$ is a free $F_{2}\langle J\rangle$ module, against $\left|V: V_{0}\right|=4$. Hence, $w=3$.

If $q \neq 3$, then Lemma 5.3 forces $q=7,|\mathfrak{B}|=7^{2}$, so that $J$ inverts $\mathfrak{S C B} / \mathscr{F}$. This forces $V$ to be a free $F_{2}\langle J\rangle$-module, against $\left|V: V_{0}\right|=4$. Hence, $q=3$.

First, suppose $|\mathfrak{B}|=9$. Then $J$ inverts $\mathfrak{S} \mathfrak{B} / \mathfrak{S}$, and we may assume that $J$ inverts $\mathfrak{B}$. Since $[V, \mathfrak{B}]$ is a free $F_{2}\langle J\rangle$-module, we get that $[\mathfrak{S}, \mathfrak{B}]=\tilde{\mathfrak{F}}$ is the central product of 2 quaternion groups on each of which $J$ induces an outer automorphism. Hence, $\left|\widetilde{\mathfrak{S}}: C_{\tilde{\mathfrak{p}}}(J)\right|=8$, against $\left|\mathfrak{S}_{\mathfrak{c}}: \mathfrak{S}_{1}\right|=4, \mathfrak{S}_{1} \subseteq \mathfrak{S}^{G}$. Hence, $|\mathfrak{B}|>9$.

Since $S_{3}$-subgroups of $S_{6}(2)$ are $Z_{3} \int Z_{3}$, we get that $\mathfrak{B}$ is non abelian of order 27 and exponent 3 . Thus, $\mathfrak{B}$ acts faithfully and irreducibly on $V$. Let $\mathfrak{B}_{0}$ be a subgroup of $\mathfrak{B}$ of order 9 , and let $\mathfrak{B}_{1}, \mathfrak{B}_{2}, \mathfrak{B}_{3}$ be the subgroups of $\mathfrak{B}_{0}$ of order 3 distinct from $\mathfrak{B}^{\prime}$. Then $C_{\mathfrak{5}}\left(\mathfrak{B}_{i}\right)$ is of order $8 i=1,2,3$, and $\mathfrak{B}^{\prime}$ acts faithfully on each $C_{\mathfrak{f}}\left(\mathfrak{B}_{i}\right)$, so $C_{\mathfrak{p}}\left(\mathfrak{B}_{i}\right)=\mathfrak{\Omega}_{i}$ is a quaternion group. Furthermore, $\mathfrak{\Omega}_{1}, \mathfrak{\Omega}_{2}, \mathfrak{\Omega}_{3}$ are the only quaternion subgroups of $\mathfrak{S}$ which admit $\mathfrak{B}_{0}$.

We assume without loss of generality that $J$ inverts $\mathfrak{B}_{1}$. Let $\mathfrak{L}=\mathfrak{S} \mathfrak{B}\langle J\rangle$. Then $\mathfrak{S} \cdot C_{\mathbb{R}}^{*}\left(\mathfrak{B}_{1}\right)=\mathfrak{S C B}_{0}\langle J\rangle$, so $J$ normalizes $\mathfrak{Q}_{1}$ and interchanges $\mathfrak{\Omega}_{2}$ and $\mathfrak{\Omega}_{3}$. Since $\left|\mathfrak{S}_{2}: \mathfrak{S}_{1}\right|=4$, we get that $J$ centralizes $\mathfrak{\Omega}_{1}$ and that $\boldsymbol{C}_{\mathfrak{\Omega}_{2} \mathfrak{a}_{3}}(J)$ is elementary of order 8. Hence, $\mathfrak{S}_{1}=\mathfrak{\Omega}_{1} \times \mathfrak{S}_{2}$, where $\mathfrak{S}_{2}$ is elementary of order 4 .

By symmetry, $\Re_{1}$ is the direct product of a quaternion group and a four-group, so $\Omega_{2}$ is quaternion.

Now $C_{\mathfrak{R}}^{*}\left(\mathfrak{B}_{1}\right)=\mathfrak{Q}_{1} \mathfrak{B}_{0}\langle J\rangle$, so that $J$ normalizes $\mathfrak{B}_{0}$. Since $N_{\mathfrak{R}}\left(\mathfrak{B}_{0}\right)=$ $\langle I\rangle \mathfrak{B}\langle J\rangle$, we get that $J$ normalizes $\mathfrak{B}$.

Suppose $\left|\mathscr{S}_{1} \cap \Re_{1}\right| \leqq 2$. Since $\left[\mathfrak{S}_{1}, \Re_{1}\right] \subseteq \mathscr{S}_{1} \cap \Re_{1}$, it follows that $\Re_{2}$ has an element $X$ of order 4 such that $\mathfrak{Q}_{1} \cap C(X) \supset\langle I\rangle$. Hence, $\left\langle X, \mathfrak{B}_{1}\right\rangle$ centralizes $\mathfrak{\Omega}_{1} \cap C(X)$; since $X^{2}=J,\left\langle X, \mathfrak{B}_{1}\right\rangle$ contains a $S_{3^{-}}$ subgroup of $\mathbb{R}$, against $C_{\mathfrak{p}}(\mathfrak{B})=\langle I\rangle$. Hence, $\left|\mathfrak{S}_{1} \cap \Re_{1}\right| \geqq 4$. Since $I \notin \mathfrak{S}_{1} \cap \Re_{1}$, it follows that $\boldsymbol{C}_{\mathfrak{f}}(J)=\mathfrak{Q}_{1} \times\left(\mathfrak{S}_{1} \cap \Re_{1}\right)$.

Since $J$ normalizes $\mathfrak{B}, J$ centralizes $\mathfrak{B}^{\prime}$, so $\mathfrak{B}^{\prime}$ normalizes $C_{\mathfrak{f}}(J)$, so $\mathfrak{B}^{\prime}$ normalizes $\Omega_{1}\left(C_{\mathfrak{f}}(J)\right)=\langle I\rangle \times \mathfrak{S}_{1} \cap \mathfrak{R}_{1}$. Hence, $\mathfrak{\Omega}^{\prime}$ normalizes $\mathfrak{F}=$ $\left\langle I, \mathfrak{S}_{1} \cap \Re_{1}, J\right\rangle$, an elementary group of order 16 , and [₹, $\left.\mathfrak{B}^{\prime}\right]$ is a fourgroup such that $\mathfrak{F}=\langle I, J\rangle \times\left[\mathfrak{F}, \mathfrak{B}^{\prime}\right]$.

Let $\mathfrak{S}_{0}=N_{\Im}(\mathfrak{F})$. Since $\mathfrak{\Omega}_{2} \mathfrak{N}_{3}$ normalizes $\mathfrak{F}$, it follows that $\mathfrak{F} \supset$ $\left\langle\mathfrak{S}_{1} \cap \Re_{1}, I\right\rangle \supset\langle I\rangle \supset 1$ is a composition series for $\mathfrak{F}$ as $\mathfrak{S}_{0}$-module. By symmetry, $\mho \supset\left\langle\mathfrak{S}_{1} \cap \Re_{1}, J\right\rangle \supset\langle J\rangle \supset 1$ is a composition series for $\mathfrak{F}$ as $N_{\Im^{G}}(\widetilde{\mho})$-module. Hence, $N(\mathfrak{F})$ acts irreducibly on $\mathfrak{\mho}$. Since $\mathfrak{F}=\langle I, J\rangle \times$ $\left[\mathfrak{F}, \mathfrak{B}^{\prime}\right]$, it follows that a $S_{3}$-subgroup of $A(\mathfrak{F})$ normalizes $\langle I, J\rangle$ and [₹, $\left.\mathfrak{B}^{\prime}\right]$. Since $\boldsymbol{A}_{\oplus}(\mathfrak{F})$ is solvable, it follows that $\boldsymbol{A}_{\oplus}(\mathfrak{F})$ is a 2, 3-group. Since $\widetilde{F}$ is irreducible, $O_{2}\left(A_{\mathscr{\Theta}}(\mathfrak{F})\right)=1$, so $\left|A_{\circledast}(\mathfrak{F})\right|=2^{a} \cdot 3^{2}$, with $a \geqq 2$. 
Hence, $\boldsymbol{A}_{\oplus}(\mathfrak{F})$ is 3 -closed, and $\langle I, J\rangle$, [₹, $\left.\mathfrak{B}^{\prime}\right]$ are the only four-subgroups of $\mathfrak{F}$ which admit $\boldsymbol{O}_{3}\left(\boldsymbol{A}_{\circlearrowleft}(\mathfrak{F})\right)$. This is not the case, since $\left[\mathfrak{Q}_{2} \mathfrak{\Omega}_{3}, J\right]=$ $\mathfrak{S} \cap \mathfrak{F}$ is elementary of order 8 . The proof is complete.

Lemma 13.67. Suppose $X \in \Im$. Let $\mathfrak{S}_{1}=C_{\mathfrak{\vartheta}}(X)$. Then the following hold:

(a) $И\left(\mathfrak{S}_{1} ; 2^{\prime}\right)$ is trivial.

(b) $\mathfrak{S}_{1} \in \mathscr{L}^{*}(\mathbb{S})$.

Proof. Suppose (a) is false. Let $\mathfrak{Q}$ be of minimal order in $И\left(\mathscr{S}_{1} ; 2^{\prime}\right)$ subject to $\mathfrak{Q} \neq 1$. Then $\mathfrak{D}$ is an elementary $q$-group for some odd prime $q$, and $\mathfrak{S}_{1}$ acts irreducibly on $\mathfrak{\Omega}$. If $\mathfrak{\subseteq} \subseteq$, then $\left[\mathfrak{S}_{1}, \mathfrak{Q}\right] \subseteq$ $\mathfrak{S} \cap \mathfrak{Q}=1$, so that $\mathfrak{D}$ stabilizes $\mathfrak{S}_{\mathcal{C}} \supset \mathfrak{S}_{1} \supset 1$, so that $\mathfrak{Q}=1$. We may

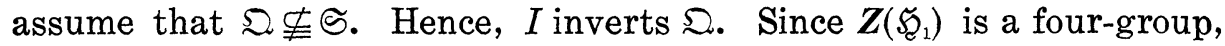
we can choose an involution $\boldsymbol{Z}$ of $\boldsymbol{Z}\left(\mathfrak{S}_{1}\right)$ which centralizes $\mathfrak{Q}$. Let $\mathfrak{E}=$ $C(Z)$. By Lemma 13.1, we get $\boldsymbol{O}_{2^{\prime}}(\mathfrak{S})=1$. Let $\mathfrak{\Im}_{2}$ be a $S_{2^{-}}$subgroup of $\sqrt{ }$ which contains $\mathscr{S}_{1}$ and let $\tilde{\mathfrak{I}}$ be a $S_{2}$-subgroup of $\mathbb{S}$ which contains $\mathfrak{F}_{2}$. Let $T$ be the central involution of $\tilde{\mathfrak{I}}$. Then $T$ centralizes $\mathfrak{S}_{1}$, so $T \in Z\left(\mathscr{F}_{1}\right) \leqq \mathfrak{F}$, by Lemma 13.62. By Lemma 13.65, we get $T=I$.

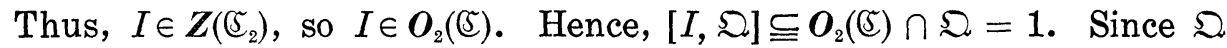
is inverted by $I$, we have the desired contradiction.

Suppose $\mathfrak{R} \in \mathscr{S} \mathscr{O} \mathscr{L}(\mathbb{S}), \mathfrak{S}_{1} \subseteq \mathfrak{N} \nsubseteq \mathfrak{S}$, and $\mathfrak{R}$ is minimal with this property. Let $\mathfrak{N}_{2}$ be a $S_{2}$-subgroup of $\mathfrak{N}$ which contains $\mathfrak{S}_{1}$. Then $\mathfrak{R}_{2} \subseteq \mathfrak{S}^{G}$ for some $G \in \mathbb{B}$, so $\mathfrak{S}_{1} \subseteq \mathfrak{S}^{G}$. By Lemma 13.63, we get $\mathfrak{S}^{G}=\mathfrak{S}$. Hence, $\mathfrak{N}_{2} \subset \mathfrak{N}$. By minimality of $\mathfrak{N}$, we have $\mathfrak{N}=\mathfrak{N}_{2} \mathfrak{Q}$ for some $q$ group $\mathfrak{\Omega}$. By $\left(\right.$ a), $\boldsymbol{O}_{2^{\prime}}(\mathfrak{R})=1$. Let $\mathfrak{L}=\boldsymbol{O}_{2}(\mathfrak{R})$. Then $I \in \mathbb{R}$, since $I \in \boldsymbol{Z}\left(\mathfrak{R}_{2}\right)$. Hence, $\mathfrak{S}_{1} \mathfrak{Q} / \mathbb{R} \cong \mathfrak{F}_{1} / \mathfrak{F}_{1} \cap \mathbb{Q}$ is elementary. By minimality of $\mathfrak{N}$, we get that $\mathfrak{D}$ is cyclic and that $\mathfrak{R}_{2}=\mathfrak{R} \mathfrak{S}_{1},\left|\mathfrak{S}_{1}: \mathfrak{S}_{1} \cap \mathbb{R}\right| \leqq 2$. Let $\mathfrak{S}_{2}=\mathfrak{S}_{1} \cap \mathfrak{R}$, so that $\left|\mathfrak{S}_{2}: \mathfrak{K}_{2}\right| \leqq 4$. Choose $Q \in \mathfrak{\Omega}-\mathfrak{S}$. Then $\mathfrak{S}_{2}^{Q} \subseteq \mathbb{R} \subseteq$

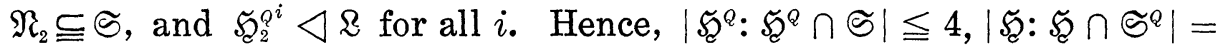
$\left|\mathfrak{S}^{Q^{-1}}: \mathfrak{S}^{Q^{-1}} \cap \mathfrak{S}\right| \leqq 4$, so Lemma 13.66 gives a contradiction.

LEMMA 13.68. $\mathfrak{S}$ is a T.I. set in (S).

Proof. Suppose $G \in \mathbb{S}-\mathfrak{S}$ and $X$ is an involution of $\mathscr{B} \cap \mathfrak{S}^{a}$. Since $\langle I\rangle$ is weakly closed in $\mathfrak{S}$, we have $X \in \mathfrak{F}$. Hence, $C(X) \subseteq \mathfrak{S}$, since $C_{\mathfrak{5}}(X) \in \mathbb{C}^{*}(\mathbb{S})$. Hence, $\boldsymbol{C}_{\mathfrak{5} G}(X) \subseteq \mathfrak{S}$, against Lemma 13.63.

LEMMA 13.69. 5 does not contain an elementary subgroup \& of order $2^{w}$ such that

(a) $\mathbb{5} \cap \mathfrak{S}=1$,

(b) $C_{\mathfrak{g}}(E)=C_{\mathfrak{\xi}}(\mathfrak{s})$ for all $E$ in (5”.

Proof. Suppose false. Let $\mathfrak{B}=\mathfrak{B}_{1} \times \cdots \times \mathfrak{B}_{w}$ be a subgroup of $\boldsymbol{O}_{2,2^{\prime}}$ (S) such that (a) $\left|\mathfrak{B}_{i}\right|=p_{i}, p_{i}$ an odd prime, (b) (5 normalizes $\mathfrak{S}_{\mathfrak{C}}$, 
$1 \leqq i \leqq w$, (c) $\sqrt{5}$ acts faithfully on $\mathfrak{S} \mathfrak{B} / \mathfrak{S}$. Let $V=\mathfrak{S E} / \mathfrak{S}^{\prime}$. By Lemma 5.8, $V=V_{1} \times \cdots \times V_{s}$, where each $V_{i}$ is an irreducible \&-module, $\mathfrak{L}=\mathfrak{S} \mathfrak{B} \mathfrak{B}$, and where $\left|V_{i}\right| \leqq 16$, all $i$. Since $w \geqq 3, C_{\mathbb{E}}\left(V_{i}\right) \neq 1$. Let

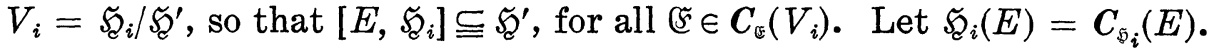
Then $\left|\mathscr{S}_{i}: \mathscr{S}_{i}(E)\right| \leqq 2$, and $\left(\mathbb{S}\right.$ centralizes $\mathscr{S}_{i}(E)$, by (b). If $\left|\boldsymbol{C}_{\mathbb{E}}\left(V_{i}\right)\right|>2$, then $\mathscr{S}_{i}=\left\langle\mathscr{S}_{i}(E) \mid E \in \boldsymbol{C}_{\mathbb{E}}\left(V_{i}\right)^{\sharp}\right\rangle$, so that $\mathfrak{S}_{i} \subseteq C(\mathbb{E})$. Suppose $\left|\boldsymbol{C}_{\mathbb{E}}\left(V_{i}\right)\right|=2$. Then $w=3,\left|V_{i}\right|=16$, and $\left|\mathscr{S}_{i}: C_{\mathfrak{S}_{i}}(\mathfrak{F})\right| \leqq 2$. This is not the case, since $\mathbb{5}$ centralizes no hyperplane of $V_{i}$. Hence, $\mathfrak{S} \subseteq C_{\mathfrak{F}}(\mathfrak{F})$, against $\mathfrak{S} \cap \mathbb{E}=1$.

\section{THEOREM 13.7. Hypothesis 13.6 is not satisfied.}

Proof. Suppose false. We use the preceding notation. By the $Z^{*}$-theorem, there is $G$ in $(B)-\mathfrak{S}$ such that $J=I^{G} \in \mathfrak{S}$. First suppose that $C_{\mathfrak{5}}(J)$ contains an element $K$ of order 4 . Then $K \in \mathfrak{S}^{a}$. Also, $\left[C_{\mathfrak{Y} G}(J), K\right] \subseteq \mathfrak{S} \cap \mathfrak{S}^{G}=1$. Let $\mathfrak{F} \in \mathscr{S} \mathscr{C} \mathscr{N}\left(\mathfrak{S}^{G}\right)$ be chosen so that $\mathfrak{F}$ admits $K$. Then $\mathfrak{F}_{0}=\Omega_{1}(\mathfrak{F})$ is elementary of order $\geqq 2^{w}$. Since $C_{\mho_{0}}\left(K^{2}\right)=C_{\mho_{0}}(K)$, we get that $J=K^{2}$ centralizes $\widetilde{\mho}_{0}$. Let $\&$ be an elementary subgroup of $\mathfrak{F}_{0}$ of order $2^{w}$. Then $\mathfrak{F} \subseteq \mathbb{S}$, and $\mathfrak{F} \cap \mathfrak{S}=1$. If $E \in \mathfrak{F}^{\sharp}$, then $\left[C_{\mathfrak{F}}(E)\right.$, [] $] \subseteq \mathfrak{S} \cap \mathfrak{S}^{a}=1$. This violates Lemma 13.69. Hence, $C_{\mathfrak{F}}(J)$ is elementary. Let $V=\mathfrak{S}_{\mathfrak{C}} / \mathfrak{S}^{\prime}$. Since $V$ is elementary of order $2^{2 w}$, it follows that $\left|C_{V}(J)\right| \geqq 2^{w}$, and so $\left|C_{\mathfrak{S}}(J)\right| \geqq 2^{w}$. Let $\mathfrak{F}_{0}$ be a subgroup of $C_{\mathfrak{F}}(J)$ of order $2^{w}$, and let $\mathbb{F}=\mathfrak{F}_{0}^{G^{-1}}$. Since $\mathfrak{F}_{0} \subseteq \mathfrak{S}^{a}$, we have $\mathfrak{5} \subseteq \mathfrak{S}$. If $\mathfrak{F} \cap \mathfrak{S} \neq 1$, then since $\mathfrak{F} \subseteq \mathfrak{S}^{G^{-1}}$, we get $\mathfrak{S}=\mathfrak{S}_{\mathfrak{C}}{ }^{-1}$, $G \in \mathfrak{S}$, against our choice of $G$. Hence, $\mathbb{F} \cap \mathfrak{S}=1$. If $E \in \mathfrak{F}^{\sharp}$, then

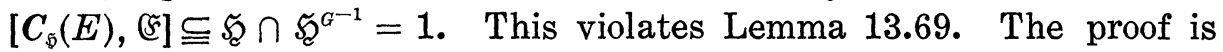
complete.

THEOREM 13.8. $\quad e(\$ S) \leqq 2$.

Proof. Suppose false. Then Hypothesis 13.1 is satisfied. Let $\mathfrak{M}$ be the subgroup given at the conclusion of Lemma 13.3. By Theorem 13.3, every normal abelian subgroup of $\mathfrak{M}$ is generated by 2 elements. Suppose $\mathfrak{B}$ is a non cyclic normal abelian subgroup of $\mathfrak{M}$. Choose $p \in \sigma$. Then $\boldsymbol{C}_{\mathfrak{n}}(\mathfrak{B})$ contains an element of $\mathscr{A}(p)$, so $C(B) \subseteq \mathfrak{M}$ for all $B$ in $\mathfrak{B}^{\sharp}$. Thus, Hypothesis 13.3 is satisfied, against 13.4. We conclude that every normal abelian subgroup of $\mathfrak{M}$ is cyclic. Theorems 13.5, 13.6, 13.7 yield a contradiction. The proof is complete.

Received November 14, 1971.

UNIVERSITY OF CAMBRIDGE 


\section{PACIFIC JOURNAL OF MATHEMATICS}

\section{EDITORS}

RICHARD ARENS (Managing Editor)

University of California

Los Angeles, California 90024

\author{
R. A. Beaumont \\ University of Washington \\ Seattle, Washington 98105
}

J. Dugundu*

Department of Mathematics

University of Southern California

Los Angeles, California 90007

D. Gilbarg and J. Milgram

Stanford University

Stanford, California 94305

\section{ASSOCIATE EDITORS}
E. F. BECKENBACH
B. H. NeumanN
F. WOLF
K. YosHIDA

\section{SUPPORTING INSTITUTIONS}

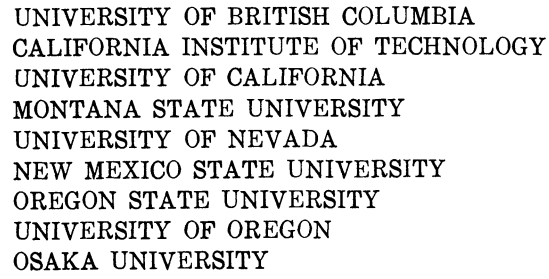

UNIVERSITY OF BRITISH COLUMBIA CALIFORNIA INSTITUTE OF TECHNOLOGY

UNIVERSITY OF CALIFORNIA

MONTANA STATE UNIVERSITY

UNIVERSITY OF NEVADA

NEW MEXICO STATE UNIVERSITY

OREGON STATE UNIVERSITY

UNIVERSITY OF OREGON

OSAKA UNIVERSITY

\author{
UNIVERSITY OF SOUTHERN CALIFORNIA \\ STANFORD UNIVERSITY \\ UNIVERSITY OF TOKYO \\ UNIVERSITY OF UTAH \\ WASHINGTON STATE UNIVERSITY \\ UNIVERSITY OF WASHINGTON \\ * * * \\ AMERICAN MATHEMATICAL SOCIETY \\ NAVAL WEAPONS CENTER
}

The Supporting Institutions listed above contribute to the cost of publication of this Journal, but they are not owners or publishers and have no responsibility for its content or policies.

Mathematical papers intended for publication in the Pacific Journal of Mathematics should be in typed form or offset-reproduced, (not dittoed), double spaced with large margins. Underline Greek letters in red, German in green, and script in blue. The first paragraph or two must be capable of being used separately as a synopsis of the entire paper. Items of the bibliography should not be cited there unless absolutely necessary, in which case they must be identified by author and Journal, rather than by item number. Manuscripts, in duplicate if possible, may be sent to any one of the four editors. Please classify according to the scheme of Math. Rev. Index to Vol. 39. All other communications to the editors should be addressed to the managing editor, or Elaine Barth, University of California, Los Angeles, California, 90024.

50 reprints are provided free for each article; additional copies may be obtained at cost in multiples of 50 .

The Pacific Journal of Mathematics is issued monthly as of January 1966. Regular subscription rate: $\$ 48.00$ a year (6 Vols., 12 issues). Special rate: $\$ 24.00$ a year to individual members of supporting institutions.

Subscriptions, orders for back numbers, and changes of address should be sent to Pacific Journal of Mathematics, 103 Highland Boulevard, Berkeley, California, 94708.

PUBLISHED BY PACIFIC JOURNAL OF MATHEMATICS, A NON-PROFIT CORPORATION

Printed at Kokusai Bunken Insatsusha (International Academic Printing Co., Ltd.), 270, 3-chome Totsuka-cho. Shinjuku-ku, Tokyo 160, Japan.

* C. R. DePrima California Institute of Technology, Pasadena, CA 91109, will replace J. Dugundji until August 1974.

Copyright (C) 1973 by

Pacific Journal of Mathematics

All Rights Reserved 


\section{Pacific Journal of Mathematics}

Vol. 48, No. $2 \quad$ April, 1973

Mir Maswood Ali, Content of the frustum of a simplex................

Mieczyslaw Altman, Contractors, approximate identities and factorization

in Banach algebras ................................ 323

Charles Francis Amelin, A numerical range for two linear operators ...... 335

John Robert Baxter and Rafael Van Severen Chacon, Nonlinear functionals

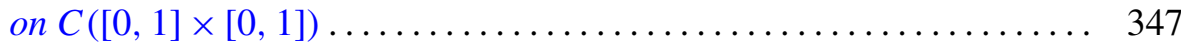

Stephen Dale Bronn, Cotorsion theories....................... 355

Peter A. Fowler, Capacity theory in Banach spaces............... 365

Jerome A. Goldstein, Groups of isometries on Orlicz spaces ........... 387

Kenneth R. Goodearl, Idealizers and nonsingular rings . ............ 395

Robert L. Griess, Jr., Automorphisms of extra special groups and

nonvanishing degree 2 cohomology ..................... 403

Paul M. Krajkiewicz, The Picard theorem for multianalytic functions . . . . 423

Peter A. McCoy, Value distribution of linear combinations of axisymmetric harmonic polynomials and their derivatives ...................

A. P. Morse and Donald Chesley Pfaff, Separative relations for

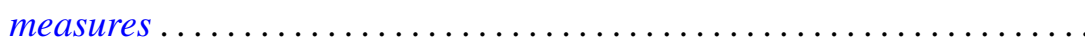

Albert David Polimeni, Groups in which $\operatorname{Aut}(G)$ is transitive on the

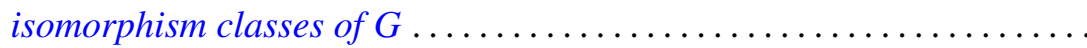

Aribindi Satyanarayan Rao, Matrix summability of a class of derived

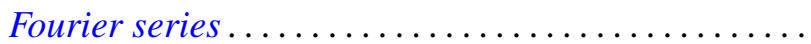

Thomas Jay Sanders, Shape groups and products

Ruth Silverman, Decomposition of plane convex sets. II. Sets associated

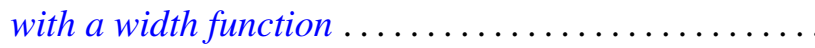

Richard Snay, Decompositions of $E^{3}$ into points and countably many flexible dendrites.............................

John Griggs Thompson, Nonsolvable finite groups all of whose local subgroups are solvable, IV ...

Robert E. Waterman, Invariant subspaces, similarity and isometric equivalence of certain commuting operators in $L_{p} \ldots$

James Chin-Sze Wong, An ergodic property of locally compact amenable

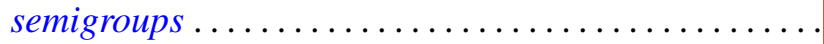

Julius Martin Zelmanowitz, Orders in simple Artinian rings are strongly equivalent to matrix rings ....................... 